\title{
Studies of the Strange Hadronic Tau Decay Tau- to KO(S) Pi- Nu-Tau Using the BaBar Detector
}

\author{
A. J. Lyon \\ Stanford Linear Accelerator Center \\ Stanford University \\ Stanford, CA 94309
}

SLAC-Report-785

Prepared for the Department of Energy

under contract number DE-AC02-76SF00515

Printed in the United States of America. Available from the National Technical Information Service, U.S. Department of Commerce, 5285 Port Royal Road, Springfield, VA 22161. 
This document, and the material and data contained therein, was developed under sponsorship of the United States Government. Neither the United States nor the Department of Energy, nor the Leland Stanford Junior University, nor their employees, nor their respective contractors, subcontractors, or their employees, makes an warranty, express or implied, or assumes any liability of responsibility for accuracy, completeness or usefulness of any information, apparatus, product or process disclosed, or represents that its use will not infringe privately owned rights. Mention of any product, its manufacturer, or suppliers shall not, nor is it intended to, imply approval, disapproval, or fitness of any particular use. A royalty-free, nonexclusive right to use and disseminate same of whatsoever, is expressly reserved to the United States and the University. 


\title{
Studies of the strange
} hadronic $\tau$ decay

\author{
$\tau^{-} \rightarrow K_{S}^{0} \pi^{-} \nu_{\tau}$ \\ using the $B A B A R$ detector
}

Andrew J Lyon

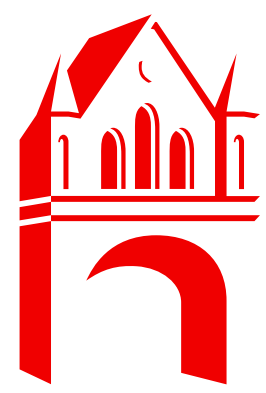

THE UNIVERSITY

of MANCHESTER

Particle Physics Group

Department of Physics and Astronomy

2004

A thesis submitted to The University of Manchester for the degree of Doctor of Philosophy in the Faculty of Science and Engineering 


\section{Contents}

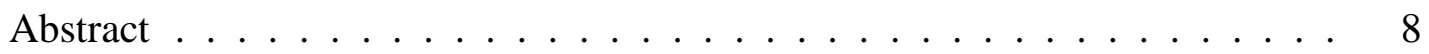

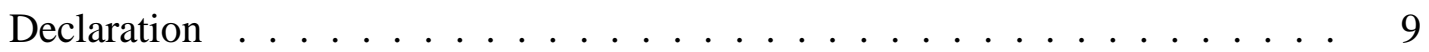

BABAR disclaimer . . . . . . . . . . . . . . . . . . . . 10

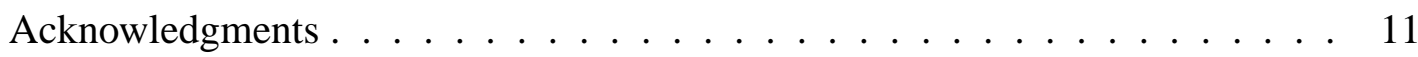

1 Tau physics at BABAR $\quad 12$

1.1 Introduction . . . . . . . . . . . . . . 12

1.2 Properties of the $\tau$ lepton . . . . . . . . . . . . 15

1.2.1 Discovery of the $\tau$ lepton . . . . . . . . . . 15

1.2.2 World average measured properties . . . . . . . . . 15

1.2.3 $\tau^{+} \tau^{-}$production at BABAR ................ 16

1.2.4 $\tau^{-} \rightarrow(\text { strange })^{-} \nu_{\tau}$ branching fractions . . . . . . . . 17

2 Theoretical issues in the $\tau$ sector $\quad 19$

2.1 Introduction . . . . . . . . . . . . . . . . 19

2.2 Theoretical overview ....................... 20

2.3 Quark mixing and the CKM matrix . . . . . . . . . . . . . 24

2.4 Theoretical framework of $\tau$ decays . . . . . . . . . . . . . . . 27

2.4.1 Hadronic $\tau$ decays . . . . . . . . . . . . . . . 27

2.4.2 Properties of the weak charged current . . . . . . . . . . 31 
2.4.3 Operator product expansion and analyticity ......... 32

2.4.4 $\tau$ hadronic decay rate ratio, $\boldsymbol{R}_{\tau} \ldots \ldots \ldots 33$

2.4.5 Spectral weights and their relation to $R_{\tau} \ldots \ldots \ldots 34$

2.4.6 OPE regime of validity for hadronic $\tau$ decay data . . . . . . . 39

2.4.7 Determination of $\boldsymbol{\alpha}_{S} \ldots \ldots \ldots \ldots$

2.4.8 Extraction of the strange quark mass, $\boldsymbol{m}_{s} \ldots \ldots . \ldots 42$

2.4.9 Longitudinal contributions to hadronic $\tau$ decay . . . . . . . . . 43

2.4.10 Determining $\left|\boldsymbol{V}_{\text {us }}\right|$ from $\boldsymbol{R}_{\tau} \ldots \ldots \ldots$. . . . . . . 47

3 The BABAR experiment 49

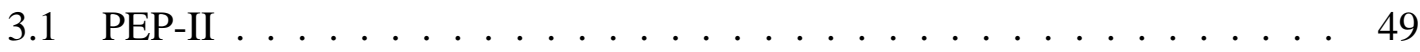

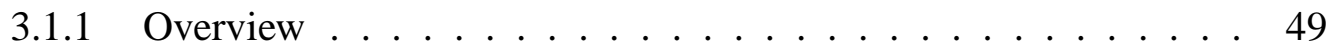

3.1.2 The interaction region ............... 51

3.1.3 Machine backgrounds ................ 52

3.1.4 PEP-II performance ................ 53

3.2 The BABAR detector . . . . . . . . . . . . . . . . . 54

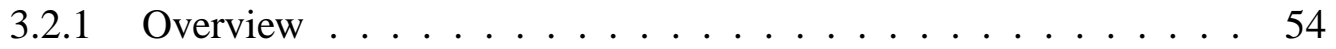

3.2.2 The BABAR coordinate system . . . . . . . . . . . . 57

3.2.3 Silicon Vertex Tracker $(\mathrm{SVT}) \ldots \ldots \ldots 58$

3.2.4 Drift Chamber $(\mathrm{DCH}) \ldots \ldots \ldots$

3.2.5 Charged particle tracking performance . . . . . . . . . 69

3.2.6 Detector of Internally Reflected Čerenkov Light (DIRC) . . . . . 72

3.2.7 Electromagnetic Calorimeter (EMC) . . . . . . . . . . . . 77 
3.2.8 Instrumented Flux Return (IFR) . . . . . . . . . . . . . 85

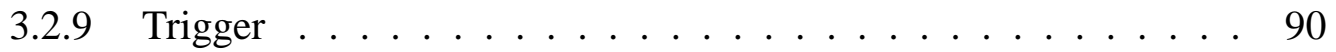

3.2.10 Online computing and data acquisition (DAQ) . . . . . . . . . 94

4 The BABAR software, analysis tools \& data quality monitoring 95

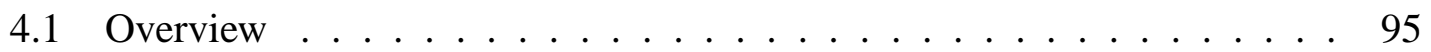

4.2 The Framework . . . . . . . . . . . . . . . . 96

4.3 Reconstruction . . . . . . . . . . . . . . . . 97

4.3.1 Available lists of charged tracks and neutral candidates . . . . . . 97

4.3.2 Reconstruction of composite candidates . . . . . . . . . . . 97

4.3.3 Available lists of composite candidates . . . . . . . . . 100

4.3.4 Particle identification: PID selectors . . . . . . . . . . . . 100

4.4 Simulation . . . . . . . . . . . . . . . . 102

4.4.1 Event generators .................... 104

4.4.2 BOGUS: detector simulation . . . . . . . . . . . . . 104

4.4.3 SimApp: detector response simulation . . . . . . . . . . 106

4.4.4 BEAR: reconstruction . . . . . . . . . . . . . 107

4.4.5 MOOSE: combining the simulation chain . . . . . . . . . . 107

4.4.6 Monte Carlo production . . . . . . . . . . . . . . . 107

4.5 Data storage and quality assurance . . . . . . . . . . . . . . 108

4.6 TauUser analysis package . . . . . . . . . . . . . . 110

4.7 Data analysis with ROOT . . . . . . . . . . . . . 111

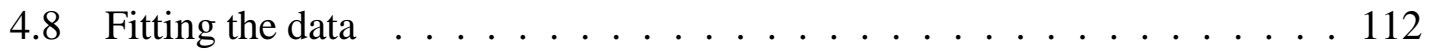


4.8 .1 Maximum likelihood . . . . . . . . . . . . . . . . . . . . 112

4.8.2 RooFit: an interface to Minuit . . . . . . . . . . . . . . . 114

5 Selecting $\tau^{-} \rightarrow K_{S}^{0} \pi^{-} \nu_{\tau}\left(K_{S}^{0} \rightarrow \pi^{+} \pi^{-}\right)$events $\quad 116$

5.1 Physics data and Monte Carlo samples . . . . . . . . . . . . . 116

5.2 Event preselection . . . . . . . . . . . . . . . . 118

$5.2 .1 \quad$ TauUser preselection $\ldots \ldots \ldots \ldots \ldots \ldots$

5.2.2 Additional preselection - skim ntuple . . . . . . . . . 120

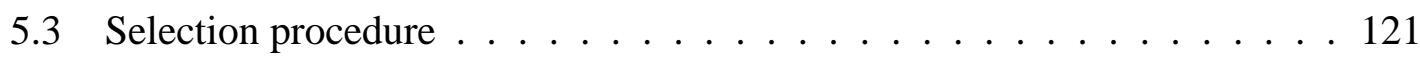

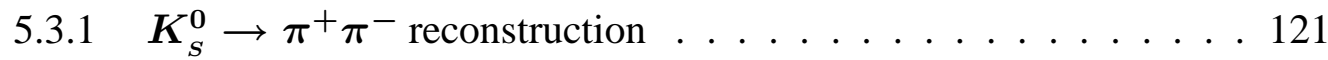

5.3.2 Lepton tagged samples . . . . . . . . . . . . . . 126

5.3.3 Identifying the bachelor pion . . . . . . . . . . . . . 127

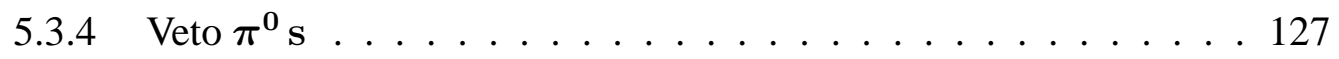

5.4 Signal efficiency $\ldots \ldots \ldots \ldots \ldots$

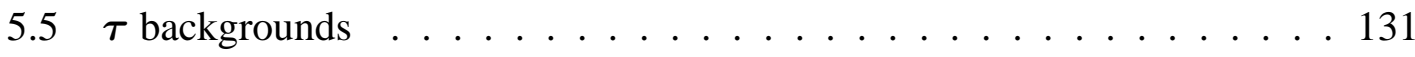

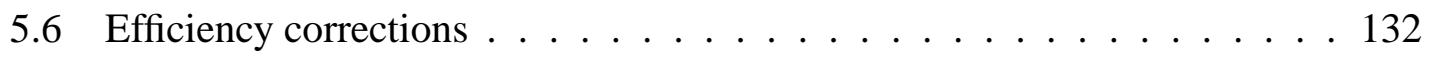

$5.6 .1 \quad K_{S}^{0}$ efficiency correction $\ldots \ldots \ldots \ldots \ldots \ldots \ldots \ldots$

5.6.2 PID-weighting Monte Carlo _ . . . . . . . . . . . . . 132

5.6 .3 Average efficiency correction $\ldots \ldots \ldots \ldots \ldots$

5.7 Systematic uncertainties $\ldots \ldots \ldots \ldots \ldots$

5.7 .1 Tracking efficiency . . . . . . . . . . . . . . . . . 139

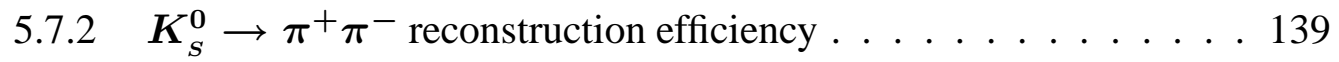

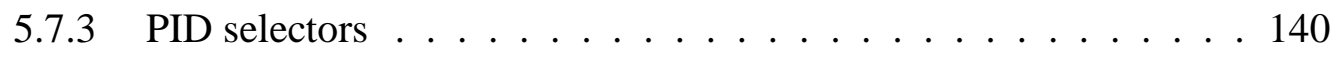


5.7.4 Luminosity and $\tau^{+} \tau^{-}$cross-section . . . . . . . . . . . . . . 141

5.7.5 Monte Carlo statistics . . . . . . . . . . . . . . . . . . 142

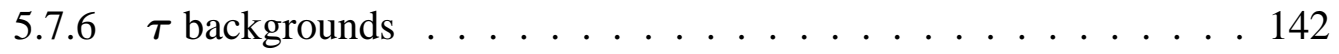

5.7.7 Systematics summary .................. 143

6 Studies of $\tau^{-} \rightarrow K_{S}^{0} \pi^{-} \nu_{\tau}$ decay

6.1 Reconstructed and identified tracks . . . . . . . . . . . . . . . . 145

6.1.1 Lepton (one-prong) tagged track . . . . . . . . . . . . . . . 145

6.1.2 Pion (bachelor) identified track . . . . . . . . . . . . 145

6.2 Selected $\boldsymbol{K}_{S}^{0}$ properties . . . . . . . . . . . . . . . . . . 147

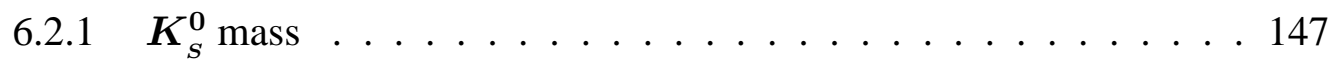

6.2.2 $\boldsymbol{K}_{s}^{0}$ momentum and angular distributions . . . . . . . . . 148

6.2.3 $\boldsymbol{K}_{S}^{0}$ decay distributions ..................... 152

$6.3 \mathcal{B}\left(\tau^{-} \rightarrow \bar{K}^{0} \pi^{-} \nu_{\tau}\right) \ldots \ldots \ldots \ldots \ldots$

$6.4 \tau^{-} \rightarrow K_{s}^{0} \pi^{-} \nu_{\tau}$ invariant mass spectra . . . . . . . . . 155

$6.4 .1 K \pi$ mass distribution $\ldots \ldots \ldots 155$

6.4.2 $\tau$ backgrounds as a function of $\boldsymbol{K} \pi$ mass . . . . . . . . . 156

6.4.3 Higher $\boldsymbol{K}^{*}$ resonances . . . . . . . . . . . . . . . . 159

6.5 Summary and outlook . . . . . . . . . . . . . . . 170

A Sensitivity of the OPE integrals to $m_{s}$

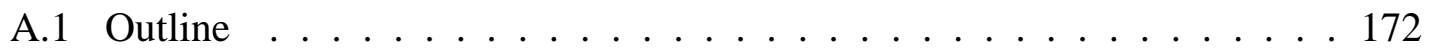

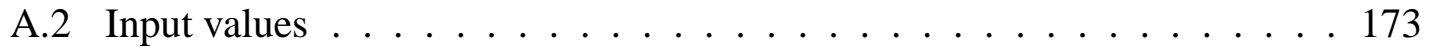

A.3 Results............................. 174 
A.4 Convergence behaviour of the OPE $D=2$ integrals $\ldots \ldots . \ldots 176$

B KK2 $f$ Monte Carlo branching fractions 


\section{Abstract}

A study of the decay $\tau^{-} \rightarrow K_{S}^{0} \pi^{-} \nu_{\tau}\left(K_{S}^{0} \rightarrow \pi^{+} \pi^{-}\right)$using the BABAR detector is presented. Using $124.4 \mathrm{fb}^{-1}$ of data we measure

$$
\mathcal{B}\left(\tau^{-} \rightarrow \bar{K}^{0} \pi^{-} \nu_{\tau}\right)=(0.830 \pm 0.005 \text { (stat) } \pm 0.042 \text { (syst) }) \%
$$

which is the world's most precise measurement to date of this branching ratio, and is consistent with the current world average. This preliminary result, unlike most of the $\mathcal{B}\left(\tau^{-} \rightarrow \bar{K}^{0} \pi^{-} \nu_{\tau}\right)$ measurements already published, is systematics dominated and so the biggest future improvement to this number should come from reducing the systematic uncertainties in the analysis.

A study of the $K \pi$ mass spectrum, from which the strange $(K \pi)$ spectral function can be measured, reveals excess contributions above the $K^{*}(892)$ tail at higher $K \pi$ mass. Whilst in the past this has been thought to be due to $K^{*}(892)-K^{*}(1410)$ interference, we find that the $K^{*}(1410)$, whose branching ratio to $K \pi$ is approximately $7 \%$, seems insufficient to explain the excess mass observed in the data. Instead, we perform a fit using a $K^{*}(892)-K^{*}(1680)$ interference model and find better agreement. The discrepancy that remains could be due to an $s$-wave contribution to the interference that is not parameterized in the model used, and/or detector smearing that is not accounted for in our fit. We also attempt to find an $s$-wave contribution to the $K \pi$ mass spectrum by searching for an $s p$-interference effect. Whilst we find a hint that such an effect exists, we have neither the confidence in the statistics nor systematics in the higher $K \pi$ mass region to announce an observation. We conclude that it would be a worthwhile study to pursue. 


\section{Declaration}

No portion of the work referred to in this thesis has been submitted in support of an application for another degree or qualification of this or any other university or other institute of learning.

Copyright in text of this thesis rests with the Author. Copies (by any process) either in full, or of extracts, may be made only in accordance with instructions given by the Author and lodged in the John Rylands University Library of Manchester. Details may be obtained from the Librarian. This page must form part of any such copies made. Further copies (by any process) of copies made in accordance with such instructions may not be made without the permission (in writing) of the Author.

The ownership of any intellectual property rights which may be described in this thesis is vested in the University of Manchester, subject to any prior agreement to the contrary, and may not be made available for use by third parties without the written permission of the University, which will prescribe the terms and conditions of any such agreement.

Further information on the conditions under which disclosures and exploitation may take place is available from the Head of the Department of Physics and Astronomy. 


\section{$B A B A R$ disclaimer}

$B A B A R$ theses are not reviewed by the BABAR collaboration and are therefore not considered official BABAR publications. 


\section{Acknowledgments}

I have been very priviledged to have worked with some of the very best physicists during my $\mathrm{PhD}$, whilst at SLAC, in Manchester or away at conferences. Moreover, I have made a number of great friends. Please indulge me while I thank a few people in particular.

My analysis was inspired by the Tau02 [1] talk given by Kim Maltman [2]. I thank Kim for presenting the initial idea to me and taking the time to exchange emails with me on a number of occasions in order that I understand the theory and the measurements that the theorists seek from experiment. Kim has been a great help and very encouraging towards this analysis - thanks Kim! I should also like to thank Matthias Jamin with whom I discussed this analysis at the BABAR collaboration meeting in Elba [3]. Following on from that discussion Matthias went away and calculated the $s$-wave hadronic $\tau$ decay branching fraction for me.

There are many great experimentalists that I am keen to thank, and inevitably I am likely to forget some. If I do, please accept my appologies: you know who you are, and thanks! However, I am unlikely to forget the considerable efforts contributed by Bill Dunwoodie. There have been a number of times that I would have been lost without him (actually, some that I have been lost with him, but that's another story!). Thanks Bill for all your help! From the $\tau$ days, I should like to thank Mike Roney, Fabrizio Salvatore, Swagato Banerjee, Olya Ignova, Carsten Hast..... Very importantly, I'd like to thank Jenny Williams for working on TauUser and running a number of ntuple production runs. (Jenny gets a mention in the beer section too. Very multifaceted is Jenny!) From the EMC simulation days, I'd like to thank Bill Lockman, Dennis Wright and David Williams in the simulation group, and Martin Kocian and Helmut Marsiske from the EMC group. From the PID group I'd like to thank Thorsten Brandt, Francesco Fabozzi and Jens Schubert for making the PID tables I use. I'd like to thank all in Manchester, particularly Roger Barlow, George Lafferty, Fred Loebinger, Jeff Forshaw.....

Now to the friends. Thanks to Phil Clark, Gary Taylor, Henning Flächer, Emmanuel ("Manny") Olaiya, Jamie Boyd, Nick Barlow, Wahid Bhimjee, Fergus Wilson, Stephen Gowdy, Richard Sloane, Adrian Bevan, Jon Coleman, Jim Wetherall, Ed Hill, Eric Charles, Alex Romosan, Jenny Williams, Mark Hodgkinson, Jamie Gaillard, Tom Latham, Kelly Ford, Sian Morgan..... all of whom have kept me company at some point along the way whilst I participate in a number of vices, mostly beer related, that I (we!!) have. Some have also kept me company on a number of fi eld trips, particularly to Las Vegas, Victoria (BC), St Croix, Hawaii, Elba, etc. They were some good times!

Thanks to PPARC for the funding and support, particularly Andy McKinna with whom I've exchanged a number of emails over the past three years.

Last but by no means least, let me express my appreciation for the invaluable support of my supervisor (and friend) John Allison. I think we've made it! (At last I hear you cry?!) 


\section{1 \\ Tau physics at $B A B A R$}

\subsection{Introduction}

Hadronic (semileptonic) $\tau$ decays provide a clean laboratory for studying the hadronic weak current. This is because there is no evidence to suggest that the $\tau$ lepton has substructure and it is the only lepton sufficiently massive to decay into hadrons. Hadronic products from $\tau$ decays give access to the light quark vector $(V)$ and axial-vector $(A)$ spectral functions, which give insight into the dynamics of QCD at intermediate scales as well as provide tests of the Standard Model [4-6] itself. Decays of the $\tau$ lepton are therefore important in determining the charged hadron decay constants and spectral functions. For decays with an overall net change of strangeness, $S U(3)_{f}$ symmetry breaking can be used to determine the Cabibbo-Kobayashi-Maskawa (CKM) matrix [7,8] element magnitude $\left|V_{u s}\right|$, the strong coupling constant, $\alpha_{S}$, and the strange quark mass, $m_{s}$. Direct measurements of the Cabibbo angle are therefore possible from strange hadronic $\tau$ decays. These are all tests of the Standard Model and deviations from this would indicate exciting new physics. A comprehensive review of the status of $\tau$ physics is given in [9]. 
Hadrons from $\tau$ decays are produced via $W$ emission. In the decay of the $W$, fundamental quantities, called spectral functions, describe global properties of the hadronic systems. Spectral functions parameterize the transition probability of creating hadrons out of the QCD vacuum (i.e. out of the charged weak current) as a function of the hadronic mass. Essentially the spectral functions consist of the invariant mass spectra of the hadronic final states normalised to their respective branching ratios and corrected for the $\tau$ phase space and decay kinematics. $\tau$ spectral functions are both experimentally and theoretically well defined. They provide information on hadron dynamics in a mass region that is dominated by resonances and complements the simpler asymptotic QCD regime. Several models have been proposed to take account of complications that arise in the dynamics in the final states with many hadrons with different possible resonance production. These models are based either on $S U(3)_{f}$ symmetry breaking or chiral Lagrangians; a useful summary of both techniques can be found in [10].

Relative to non-strange ( $u d$ ) currents, strange (us) currents of $\tau$ decays are suppressed by an amount $\left(\left|V_{u s}\right| /\left|V_{u d}\right|\right)^{2} \simeq \tan ^{2} \theta_{C}$, where $\left|V_{u d}\right|$ and $\left|V_{u s}\right|$ are absolute CKM matrix elements and $\theta_{C}$ is the Cabibbo angle. Resonant decay dominates these currents: the strange vector current is dominated by a $K^{*}$ resonance which decays to $K \pi$ and the strange axialvector current by the $K_{1}$ which decays mostly via $K \rho$ and $K^{*} \pi$ to $K \pi \pi$.

The BABAR detector is an excellent apparatus for studying $\tau$ decays. Not only is it able to separate pions and kaons excellently, but also has a high cross-section for $\tau$ production making it ideally suited to making high precision measurements. Moreover, $K_{S}^{0} \rightarrow \pi^{+} \pi^{-}$ can be unambiguously identified with little background without relying on kaon identification. In the data-taking periods between October 1999 and June 2003, BABAR has accumulated $125.4 \mathrm{fb}^{-1}$ of $e^{+} e^{-}$collision data. Since the cross-section for $\tau^{+} \tau^{-}$production is similar to that of $B \bar{B}$ production, there is a similar number of $e^{+} e^{-} \rightarrow \tau^{+} \tau^{-}$ events, i.e. approximately 110 million $\tau^{+} \tau^{-}$pairs. These data are mainly on the $\Upsilon(4 S)$ resonance energy $(10.58 \mathrm{GeV})$, although a smaller fraction are off-resonance $(40 \mathrm{MeV}$ below the $\Upsilon(4 S)$ ). The " 12 -series processed Run $1+2+3$ data set" contains $113.3 \mathrm{fb}^{-1}$ on- 
resonance and $12.1 \mathrm{fb}^{-1}$ off-resonance data and its definition is now frozen. This analysis uses that complete data set.

At the Tau02 conference [1] a review of $\tau$ physics and an outlook were presented. The most relevant contributions for this analysis can be found in [2,11-13]. Using the aforementioned data set, we make measurements relevant to some of the studies suggested in these papers by studying the decay $\tau^{-} \rightarrow K_{S}^{0} \pi^{-} \nu_{\tau}\left(K_{S}^{0} \rightarrow \pi^{+} \pi^{-}\right)^{1}$. This allows us to make the world's most precise measurement of the $\tau^{-} \rightarrow \bar{K}^{0} \pi^{-} \nu_{\tau}$ branching ratio. A study of the $K \pi$ invariant mass spectrum from $\tau^{-} \rightarrow K_{S}^{0} \pi^{-} \nu_{\tau}$ decays is also conducted with fits to the dominant $K^{*}(892)^{-}$and smaller contributions from higher $K^{*}$ resonances using a $p$-wave interference parameterization. From the experimentally measured $\tau$ strange spectral functions, it is possible to create $S U(3)_{f}$ flavour breaking differences to extract $\alpha_{S}$ and $m_{s}$. Using such measurements the theoretical QCD sum rules can be tested. In recent years, measurements of $\tau$ decay branching fractions to strange hadronic final states and studies of the strange spectral functions have been conducted by ALEPH [14-21], CLEO [22-29] and OPAL [30-34], but have often been limited by statistics. With BABAR's larger data sample we seek to improve upon their precision.

A search for the chirally-suppressed $s$-wave $K_{0}^{*}(1430)^{-}$, a state not yet observed in hadronic $\tau$ decay data, is also discussed. Theoretically, the operator product expansion (OPE) representations of the longitudinal contributions are known to be poorly converging [35]. A measurement of the longitudinal $K_{0}^{*}(1430)^{-}$spectral distribution would enable a longitudinal subtraction to be performed so that theorists can work with the "wellbehaved" $0+1$ sum. The $K_{0}^{*}(1430)^{-}$is experimentally challenging to measure since a partial wave analysis (PWA) needs to be employed to separate it from the $p$-wave $K^{*}$ resonances that are in a similar mass region, for example $K^{*}(1410)^{-}$.

Other interests concerning the $K_{0}^{*}(1430)^{-}$resonance come from its use as a $C P$ violation mechanism in the $\tau$ sector [36]. The $K_{0}^{*}(1430)^{-}$, due to $s p$-interference, may well show

\footnotetext{
${ }^{1}$ Throughout this thesis, whenever a mode is given, the charge conjugate is also implied.
} 
the first evidence of $C P$ violation in the $\tau$ sector. CLEO reported on such a study in the $K \pi$ system [37], but so far in this system no experimental evidence for $C P$ violation exists.

\subsection{Properties of the $\tau$ lepton}

\subsubsection{Discovery of the $\tau$ lepton}

The $\tau$ lepton was first discovered in 1975 at the Stanford Linear Accelerator Center by M. Perl et al using the MARK I detector and SPEAR $e^{+} e^{-}$collider [38]. This discovery gave the first evidence for a third generation of elementary fermions, later observed too in the quark sector with the discovery of the bottom and top quarks. Since 1975, the $\tau$ lepton has had many of its properties studied.

\subsubsection{World average measured properties}

Some of the properties of the $\tau$ lepton according to Particle Data Group (PDG) 2002 [39] are listed in Table 1.1.

\begin{tabular}{|c||c|}
\hline Property & PDG values \\
\hline \hline$J$ & $\frac{1}{2}$ \\
\hline$m_{\tau}$ & $1776.99_{-0.26}^{+0.29} \mathrm{MeV} / c^{2}$ \\
\hline$\tau_{\tau}$ & $(290.6 \pm 1.1) \times 10^{-15} \mathrm{~s}$ \\
\hline$c \tau_{\tau}$ & $87.11 \mu \mathrm{m}$ \\
\hline
\end{tabular}

Table 1.1: Properties of the $\tau$ lepton: spin $(J)$, mass $\left(m_{\tau}\right)$, mean lifetime $\left(\tau_{\tau}\right)$ and the corresponding mean decay length $\left(c \tau_{\tau}\right)$. The PDG values are taken from PDG 2002 [39].

The $\tau$ lepton is the only lepton sufficiently massive to decay into hadrons. At threshold, 
the cross-section for $\tau$ production from $e^{+} e^{-}$collisions is given by [40]

$$
\sigma_{\tau}=\frac{4 \pi \alpha^{2}}{3 s} \frac{\beta\left(3-\beta^{2}\right)}{2} F_{C},
$$

where $\alpha$ is the electromagnetic coupling constant and $\beta=\left(1-4 m_{\tau}^{2} / s\right)^{1 / 2}$, with $\tau$ mass $m_{\tau}$ and where $s$ is the usual Mandelstram variable [41] equal to the centre of mass energy squared. $F_{C}$ is the Coulomb term which encompasses the attraction between the $\tau^{+}$and $\tau^{-}$. It is given by:

$$
F_{C} \sim \frac{\pi \alpha}{\beta\left(1-e^{-\pi \alpha / \beta}\right)}
$$

High resolution silicon vertex detectors provide a means of measuring the $\tau$ lifetime. $B A B A R$ has begun to measure the $\tau$ lifetime, $\tau_{\tau}$, and has a preliminary result of [42]

$$
\tau_{\tau}=290.8 \pm 1.5(\text { stat }) \pm 1.6 \text { (syst) fs, }
$$

which corresponds well to the PDG average value (Table 1.1). A $\tau$ of momentum $4 \mathrm{GeV} / c$ travelling for 5 lifetimes travels about $1 \mathrm{~mm}$.

\subsection{3 $\tau^{+} \tau^{-}$production at $B A B A R$}

Figure 1.1 shows the $\tau^{+} \tau^{-}$production cross-section through the process $e^{+} e^{-} \rightarrow \tau^{+} \tau^{-}$ as a function of the centre of mass energy. Threshold production means that $s=m_{\tau}^{2}$ so that $\beta=0$. Therefore, Eq. (1.1) becomes

$$
\sigma_{\tau}^{\text {threshold }}=\frac{\pi^{2} \alpha^{3}}{2 m_{\tau}^{2}},
$$

ignoring initial state radiation (ISR), i.e. the radiation of photons by the $e^{+}$and $e^{-}$, which decreases $\sigma_{\tau}^{\text {threshold }}$. Inserting the BABAR energy $(\sqrt{s}=10.58 \mathrm{GeV})$ into Eq. (1.1) and Eq. (1.2) gives an estimate of the $\tau^{+} \tau^{-}$production cross-section to be $0.94 \mathrm{nb}$. In practice the cross-section is $0.89 \mathrm{nb}$ on-resonance and $0.90 \mathrm{nb}$ off-resonance, i.e. $40 \mathrm{MeV}$ below the $\Upsilon(4 S)$ resonance energy. These numbers differ from the $0.94 \mathrm{nb}$ estimate due to radiative effects, such as ISR. For $\tau$ physics at BABAR both the on-resonance and offresonance data can be used. 


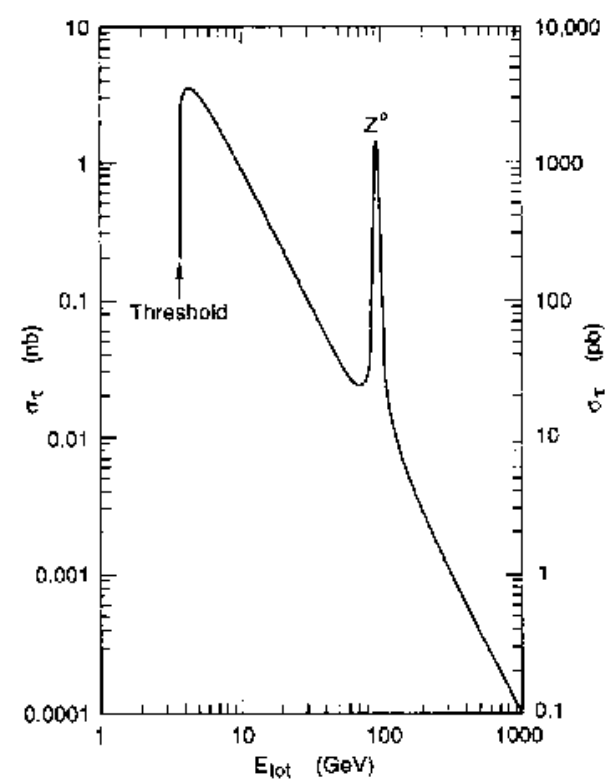

Figure 1.1: $\tau^{+} \tau^{-}$production cross-section from $e^{+} e^{-} \rightarrow \tau^{+} \tau^{-}$ignoring ISR effects which decreases $\sigma_{\tau}^{\text {threshold }}$ [40].

\subsection{4 $\tau^{-} \rightarrow(\text { strange })^{-} \nu_{\tau}$ branching fractions}

Invariant mass spectra of hadronic $\tau$ decays with an overall net change of strangeness provide access to the strange spectral function, the theoretical framework of which is discussed in the next chapter. Table 1.2 summarises the PDG 2002 branching fractions of the relevant decay channels [39]. For the $K \pi$ state of interest here, the total branching fraction is $\mathcal{B}\left(\tau^{-} \rightarrow(K \pi)^{-} \nu_{\tau}\right)=(1.340 \pm 0.050) \%$. There are two decay channels for this: $\mathcal{B}\left(\tau^{-} \rightarrow K^{-} \pi^{0} \nu_{\tau}\right)=(0.450 \pm 0.030) \%$ and $\mathcal{B}\left(\tau^{-} \rightarrow \bar{K}^{0} \pi^{-} \nu_{\tau}\right)=(0.890 \pm 0.040) \%$. In the latter case, the $\bar{K}^{0}$ flavour eigenstate mixes with its antiparticle to form one of two equally probable ${ }^{2}$ mass eigenstates, $K_{S}^{0}$ and $K_{L}^{0}$, i.e. $50 \%$ of $\tau^{-} \rightarrow \bar{K}^{0} \pi^{-} \nu_{\tau}$ are $\tau^{-} \rightarrow$ $K_{L}^{0} \pi^{-} \nu_{\tau}$ decays and $50 \%$ are $\tau^{-} \rightarrow K_{S}^{0} \pi^{-} \nu_{\tau}$ decays. Moreover, for the $\tau^{-} \rightarrow K_{S}^{0} \pi^{-} \nu_{\tau}$ channel, there are two primary decays of the $K_{S}^{0}: \mathcal{B}\left(K_{S}^{0} \rightarrow \pi^{+} \pi^{-}\right)=(68.60 \pm 0.27) \%$ and $\mathcal{B}\left(K_{S}^{0} \rightarrow \pi^{0} \pi^{0}\right)=(31.40 \pm 0.27) \%$. Other $K_{S}^{0}$ decay branching fractions are less than $\mathcal{O}\left(10^{-3}\right)$. According to PDG 2002 therefore, the decay channel reconstructed in this

\footnotetext{
${ }^{2}$ Ignoring small $C P$-violating possibilities.
} 
thesis has a branching fraction:

$$
\mathcal{B}\left(\tau^{-} \rightarrow K_{S}^{0} \pi^{-} \nu_{\tau} ; K_{S}^{0} \rightarrow \pi^{+} \pi^{-}\right)=(0.305 \pm 0.014) \%
$$

and thus a relative uncertainty of around $5 \%$. We seek to provide a high precision measurement to improve on the $\mathcal{B}\left(\tau^{-} \rightarrow \bar{K}^{0} \pi^{-} \nu_{\tau}\right)$ average, Table 1.2. With $1.1 \times 10^{8} \tau^{+} \tau^{-}$ pairs available for analysis, we have a potential sample of over $6.5 \times 10^{5} \tau^{-} \rightarrow K_{S}^{0} \pi^{-} \nu_{\tau}$ $\left(K_{S}^{0} \rightarrow \pi^{+} \pi^{-}\right)$events.

\begin{tabular}{|c|c|}
\hline Decay channel & [\%] \\
\hline$\tau^{-} \rightarrow K^{-} \nu_{\tau}$ & 0.68 \\
\hline$\tau^{-} \rightarrow K^{-} \eta \nu_{\tau}$ & 0.006 \\
\hline$\tau^{-} \rightarrow K^{-} \pi^{0} \nu_{\tau}$ & $0.450 \pm 0.030$ \\
\hline$\tau^{-} \rightarrow \bar{K}^{0} \pi^{-} \nu_{\tau}$ & $0.890 \pm 0.040$ \\
\hline$\tau^{-} \rightarrow \bar{K}^{0} \pi^{-} \pi^{0} \nu_{\tau}$ & 0.040 \\
\hline$\tau^{-} \rightarrow K^{-} \pi^{+} \pi^{-} \nu_{\tau}$ & $0.280 \pm 0.050$ \\
\hline$\tau^{-} \rightarrow K^{-} \pi^{0} \pi^{0} \nu_{\tau}$ & $0.058 \pm 0.023$ \\
\hline$\tau^{-} \rightarrow K^{*}(892) \eta \pi^{0} \nu_{\tau}$ & $0.029 \pm 0.009$ \\
\hline$\tau^{-} \rightarrow K^{-} \pi^{+} \pi^{-} \pi^{0} \nu_{\tau}$ & $0.064 \pm 0.024$ \\
\hline$\tau^{-} \rightarrow \bar{K}^{0} \pi^{-} \pi^{0} \pi^{0} \nu_{\tau}$ & $0.026 \pm 0.024$ \\
\hline$\tau^{-} \rightarrow K^{-} \pi^{0} \pi^{0} \pi^{0} \nu_{\tau}$ & $0.037 \pm 0.021$ \\
\hline$\tau^{-} \rightarrow \bar{K}^{0} \pi^{-} \pi^{+} \pi^{-} \nu_{\tau}$ & $0.023 \pm 0.020$ \\
\hline TOT & $2.940 \pm 0.099$ \\
\hline
\end{tabular}

Table 1.2: Branching fractions of the $\tau$ decay channels with an overall net change of strangeness from PDG 2002 [39]. The highlighted row contains the $\tau^{-} \rightarrow K_{S}^{0} \pi^{-} \nu_{\tau}\left(K_{S}^{0} \rightarrow \pi^{+} \pi^{-}\right)$channel that is reconstructed here. 


\section{Theoretical issues in the $\tau$ sector}

\subsection{Introduction}

In this chapter there is a thorough description of the theoretical issues regarding $\tau$ physics, particularly involving decay modes that have strange hadronic final states. Some of these descriptions go beyond the scope of the analysis that is presented later but are the ultimate aims of such studies that have begun at BABAR. In order to motivate these studies the theoretical background is discussed here.

This chapter is organised as follows: Section 2.2 provides an overview of the theoretical issues related to hadronic $\tau$ physics, details where these align with the scope of the analysis presented in Chapter 6, and outlines what would hope to be achieved in future studies. Throughout the rest of Chapter 2 some of the various theoretical points are expanded upon.

The analysis presented in Chapter 6 concentrates on the decay channel $\tau^{-} \rightarrow K_{S}^{0} \pi^{-} \nu_{\tau}$ $\left(K_{S}^{0} \rightarrow \pi^{+} \pi^{-}\right)$, and has two key aspects: 
$\diamond$ Measurement of $\mathcal{B}\left(\tau^{-} \rightarrow \bar{K}^{0} \pi^{-} \nu_{\tau}\right)$.

$\diamond$ Studies of the $K \pi$ invariant mass spectra.

Presented in the rest of Chapter 2 are two principal illustrations of where improvements relating to the extraction of $m_{s}$ using hadronic $\tau$ decay data can be achieved. The first comes by way of improvements in measurements of the strange spectral functions which are directly related to the invariant mass spectra of the produced hadrons. The second arises from a possible measurement of the scalar component of strange hadronic $\tau$ decays, expected to be manifest as a $K_{0}^{*}(1430)^{-}$resonance. This state has not yet been observed in $\tau$ decay data: it is expected to have a small branching fraction and it is also complicated by the need to separate it, using the spin-parity properties, from other $K^{*}$ resonances in a similar mass region. In Chapter 6 neither the translation of the mass spectra measurements into spectral functions nor a result for $\mathcal{B}\left(\tau^{-} \rightarrow K_{0}^{*}(1430)^{-} \nu_{\tau}\right)$ are presented, but two stepping-stones en route to those goals are.

\subsection{Theoretical overview}

Hadrons from $\tau$ decays are produced by the charged weak current, i.e. from the QCD vacuum. The hadronic physics factorises in these processes and so can be completely characterised for each decay channel by spectral functions as far as the total decay rate is concerned. The spectral functions are directly related to the invariant mass spectra of the hadronic final states normalised to their respective branching ratios and corrected for the $\tau$ decay kinematics. Hadronic $\tau$ decay data give access to the light quark vector $(V)$ and axial-vector $(A)$ spectral functions, and some of the terminology used in the rest of the discussion in relation to these is defined in Table 2.1. Investigations of the dynamics of quantum chromodynamics (QCD) at intermediate scales and improved determinations of certain QCD/Standard Model parameters are therefore possible.

The $\tau$ strange spectral functions, like in the non-strange sector, provide the possibility of 


\begin{tabular}{|c||c|}
\hline Hadronic final state & Property \\
\hline \hline Scalar & $J^{P}=0^{+}$ \\
\hline Pseudoscalar & $J^{P}=0^{-}$ \\
\hline Vector & $J^{P}=1^{-}$ \\
\hline Axial-Vector & $J^{P}=1^{+}$ \\
\hline Longitudinal & $J=0$ \\
\hline Transverse & $J=1$ \\
\hline
\end{tabular}

Table 2.1: Terminology used to describe hadronic final states.

following the results of such QCD studies at the $\tau$ mass scale. The strange quark mass can be determined by invoking the QCD sum rules and exploiting the difference between $u d$ and $u s$ vector and/or axial-vector correlators, whose spectral functions are measurable in hadronic $\tau$ decay.

The $\tau$ hadronic decay rate ratio, $R_{\tau}$, can be both measured experimentally (it is calculated in terms of weighted integrals involving the spectral functions, and the spectral functions are directly related to the measured invariant mass spectra of the final state hadrons) and calculated theoretically using the equivalent operator product expansion (OPE) contour integrals, as discussed in Section 2.4.5. The basic idea is that by equating these integrals using the finite energy sum rule (FESR) relationship, measurable quantities obtained from experiment (spectral functions) can be equated with calculable quantities from theory (correlators), involving the unknown parameter $m_{s}$. Consequently, $m_{s}$ can be determined, as discussed in Section 2.4.8.

Theorists ideally want the total strange spectral function determined independent of resonance structure, separated into vector and axial-vector contributions. However, experimentally it is difficult to separate into vector and axial-vector components, but there are many interesting QCD topics that can be studied using the $V+\mathrm{A}$ sum without embarking on this difficult separation. The three such applications Maltman discussed in detail are [2]: 
$\diamond$ The investigation of the nature of duality violation in QCD at intermediate scales, and its relation to the determination of $\alpha_{S}$.

$\diamond$ The extraction of the strange quark mass, $m_{s}$, from flavour breaking differences of flavour $u d$ and $u s$ data.

$\diamond$ The determination of the dimension $D=6$ term in the operator product expansion for the flavour $u d V-$ A correlator difference.

The strange quark mass, $m_{s}$, is one of the free parameters in the Standard Model and appears in many phenomenological calculations, for example the $C P$ violating kaon parameters $\varepsilon^{\prime} / \varepsilon$ [43-48]. The last bullet point concerns the evaluation of the chiral limit values of the $K \rightarrow \pi \pi$ electroweak penguin matrix elements which also provides information about the $\varepsilon^{\prime} / \varepsilon$ ratio but is not considered here; it is described in detail in [2].

In the above models, the strange $\tau$ decay rate is sensitive to $m_{s}$ which can thus be experimentally inferred. Since $m_{s}$ is relatively large with respect to the non-strange $u$ and $d$ quark masses, it is possible to break chiral symmetry; in the massless $S U(3)_{f}$ limit this would not happen. This introduces a mass dependence into the perturbative QCD prediction of the total strange hadronic width of the $\tau$. A combination of strange and nonstrange modes can be found using the total rates, along with the moments of the relevant spectral functions, such that the dominant massless perturbative contribution cancels. The strange quark mass can subsequently be derived using a combined fit without perturbative corrections using only these non-perturbative contributions.

Improving the large statistical uncertainties in us spectral data above the $K^{*}$ region provides a way of subtracting the poorly converging longitudinal part of the us vector and axial-vector correlators in the OPE representation. In turn, this provides a check of the optimisation method of the finite energy sum rule extraction of the strange quark mass [49].

There are a few reasons why improved experimental measurements are important to the FESR extraction of $m_{s}$. The first is that previous measurements of the spectral func- 
tions have been limited by statistics: in the $K^{*}$ region the uncertainties are around the 6-8\% level and above $\sim 1 \mathrm{GeV}^{2}$ they increase to around 20-30\% [2]. Improving these will therefore reduce the error on $m_{s}$ which is extracted using the experimental data. Moreover, the OPE does not handle the scalar $\left(J^{P}=0^{+}\right)$contribution well and so currently the $m_{s}$ determination suffers from a highly conservative uncertainty due to the poor understanding of this contribution on the theory side. If the corresponding scalar spectral function could be measured experimentally, it would be possible to perform a "longitudinal subtraction" which, even with a $100 \%$ uncertainty associated with it, would correspond to a much smaller uncertainty than that associated with the badly-behaved longitudinal OPE representation. In other words, any reasonable measurement of the scalar spectral function from experiment will enable theorists to use this, via the FESR relationship which equates experimental quantities with theoretical quantities, to subtract off the scalar contribution on the theory side. To date, however, the presence of a scalar contribution to strange hadronic $\tau$ decay has not been observed experimentally. The scalar contribution is expected to be manifest as $K_{0}^{*}(1430)^{-}$which decays primarily to $K \pi$. Even if not observed in the higher statistics samples available at, for example, $B$ Factory experiments such as $B A B A R$, an improvement in the branching ratio limit by a factor of 5 of this scalar resonance would benefit the $m_{s}$ measurement. Currently the limit is $\mathcal{B}\left(\tau^{-} \rightarrow K_{0}^{*}(1430)^{-} \nu_{\tau}\right)<5 \times 10^{-4}$ measured by ALEPH [20].

In Chapter 6, a stepping-stone along the path to measuring the total strange spectral function and a stepping-stone towards a possible measurement (or limit) of $\mathcal{B}\left(\tau^{-} \rightarrow\right.$ $\left.K_{0}^{*}(1430)^{-} \nu_{\tau}\right)$ are presented. The first comes from the measurement and fits to the $K \pi$ invariant mass spectra. Since the spectral functions are directly related to the invariant mass spectra, these can, in principle, be calculated. However, it is really the total strange spectral function for $\tau^{-} \rightarrow$ (strange $)^{-} \nu_{\tau}$ that is most important as far as computing the decay rate is concerned. Once the invariant mass spectra from all the $\tau^{-} \rightarrow(\text { strange })^{-} \nu_{\tau}$ channels have been measured, then it will be possible to compute the total strange spectral function and therefore compute the strange component of $R_{\tau}$, that is $R_{\tau}^{\text {strange }}$. In this analysis we only provide one component of that, which corresponds to $\tau^{-} \rightarrow(K \pi)^{-} \nu_{\tau}$ decay. 
The other strange decay channels have not yet been studied at $B A B A R$, but would be good avenues to pursue so that ultimately a BABAR measurement of $R_{\tau}^{\text {strange }}$ can be presented. Since BABAR has a higher statistics sample over the whole spectral range than have been used in all analyses currently published, we would expect to obtain a more precise measurement of $R_{\tau}^{\text {strange }}$ which would lead to a smaller uncertainty on $m_{s}$ extracted from $\tau$ decay data than is currently obtainable. Secondly, in this analysis the scalar $K_{0}^{*}(1430)^{-}$ resonance is searched for by looking for an $s p$-interference effect. Whilst we observe that some such effect might be present in our data, we have neither the confidence in the statistics nor systematics at the precision needed to announce an observation. Since, as discussed in Section 2.4.9, it is non-trivial to assign what is "resonance" and what is "rest" for the scalar system we have omitted quoting a limit on $\mathcal{B}\left(\tau^{-} \rightarrow K_{0}^{*}(1430)^{-} \nu_{\tau}\right)$. To do so at this stage would be presumptuous: it would require considerably more effort to understand the spin separation of the resonance contributions at higher $K \pi$ mass as well as other backgrounds and resolution effects, and is beyond the scope of this analysis. Again, this would be a useful study to pursue.

\subsection{Quark mixing and the CKM matrix}

Both leptons and quarks participate in weak interactions through charged $V-\mathrm{A}$ currents. The quark mixing angle (Cabibbo angle, $\theta_{C}$ ) was introduced in 1963 to account for weak decays of strange particles [7]. Prior to this the observation of decays such as $K^{+} \rightarrow \mu^{+} \nu_{\mu}$ could not be catered for theoretically as a doublet of $u d$ and $c s$ would not lend itself to a weak current coupling to a $u$ and $\bar{s}$ as it does in $K^{+} \rightarrow \mu^{+} \nu_{\mu}$; in the $u d$ and $c s$ doublet scheme only transitions between $u \leftrightarrow d$ and $c \leftrightarrow s$ would be possible. In this simple scheme lepton-quark symmetry means that the weak interactions of quark families,

$$
\left(\begin{array}{l}
u \\
d
\end{array}\right) \text { and }\left(\begin{array}{c}
c \\
s
\end{array}\right),
$$

are identical to those of the lepton families,

$$
\left(\begin{array}{c}
\nu_{e} \\
e^{-}
\end{array}\right) \text {and }\left(\begin{array}{c}
\nu_{\mu} \\
\mu^{-}
\end{array}\right) \text {. }
$$


In the Standard Model, the weak coupling constant, $g_{W}$, is the same for flavour changing transitions $u \leftrightarrow d$ and $c \leftrightarrow s$ as it is for $e^{-} \leftrightarrow \nu_{e}$ and $\mu^{-} \leftrightarrow \nu_{\mu}$, for example in muon decay $\mu^{-} \rightarrow \nu_{\mu} e^{-} \bar{\nu}_{e}$. Transitions between the different generations are forbidden at this level of simplicity. However, the observation of kaon decays, such as $K^{+} \rightarrow \mu^{+} \nu_{\mu}$, to lighter mesons meant that this could not be the whole story; such decays require the charged current $s \rightarrow u$ transition. By allowing the quarks to mix, such observations can be accounted for in the Standard Model. Cabibbo introduced the $u d^{\prime}$ doublet and $c s^{\prime}$ doublet for this purpose. The $d^{\prime}$ and $s^{\prime}$ are given by:

$$
\begin{aligned}
& d^{\prime}=d \cos \theta_{C}+s \sin \theta_{C} \\
& s^{\prime}=-d \sin \theta_{C}+s \cos \theta_{C} .
\end{aligned}
$$

The Cabibbo angle has been measured experimentally to be $12.8^{\circ}$ [39].

Quark mixing was included in the Standard Model [4-6] resulting in the CKM (CabibboKobayashi-Maskawa) matrix model of quark mixing ${ }^{1}[7,8]$. The weak quark eigenstates are different from the quark mass eigenstates and the relationship between these eigenstates is given by the elements in the CKM matrix,

$$
V=\left(\begin{array}{ccc}
V_{u d} & V_{u s} & V_{u b} \\
V_{c d} & V_{c s} & V_{c b} \\
V_{t d} & V_{t s} & V_{t b}
\end{array}\right)
$$

The $V_{i j}$ parameterize the relative strengths of the transition of "down-like" quarks $(j=$ $d, s, b)$ to "up-like" quarks $(i=u, c, t)$. For example, $V_{u d}$ represents a charge raising vertex like $d \rightarrow W^{-} u$ and $V_{u d}^{*}$ represents a charge lowering vertex, $u \rightarrow W^{+} d$. The unitarity of the CKM matrix is apparent in the standard parameterization [50]:

$$
V=\left(\begin{array}{ccc}
c_{12} c_{13} & s_{12} c_{13} & s_{13} e^{-i \delta} \\
-s_{12} c_{23}-c_{12} s_{23} s_{13} e^{i \delta} & c_{12} c_{23}-s_{12} s_{23} s_{13} e^{i \delta} & s_{23} c_{13} \\
s_{12} s_{23}-c_{12} c_{23} s_{13} e^{i \delta} & -c_{12} s_{23}-s_{12} c_{23} s_{13} e^{i \delta} & c_{23} c_{13}
\end{array}\right)
$$

where $c_{i j} \equiv \cos \theta_{i j}, s_{i j} \equiv \sin \theta_{i j}$ and $\delta$ is the complex phase.

\footnotetext{
${ }^{1}$ For the remainder of this thesis quark mixing is implied and the primes on quark mixtures are dropped to ease the notation.
} 
The diagonal elements in the CKM matrix are near unity whilst the off-diagonal elements are small, but significantly not zero. In general, a $3 \times 3$ matrix requires eighteen real numbers to define it, but the CKM matrix only requires four; for three quark generations, the matrix elements may be expressed in terms of three real angles and one complex phase. It is the complex phase that forces the condition $V \neq V^{*}$ so that $C P$ violation occurs.

A brief mention about $C P$ violation seems unavoidable as it is the main physics motivation for the $B A B A R$ experiment, although not the main thrust of this analysis. BABAR has already made the first observation of $C P$ violation in the $B$ meson system [51]. Studies are also currently underway to investigate possible $C P$ violating effects in the $\tau$ sector. These look at the $s p$-interference between the $p$-wave $\tau$ decays through a $W^{ \pm}$and the $s$-wave $\tau$ decays through a possible charged Higgs boson, $H^{ \pm}$.

A condition resulting from the Standard Model is that $V^{\dagger} V=1$ and so a number of relations among elements of the CKM matrix can be made. Three of the most useful for understanding $C P$ violation are:

$$
\begin{aligned}
V_{u d} V_{u s}^{*}+V_{c d} V_{c s}^{*}+V_{t d} V_{t s}^{*} & =0 ; \\
V_{u s} V_{u b}^{*}+V_{c s} V_{c b}^{*}+V_{t s} V_{t b}^{*} & =0 ; \\
V_{u d} V_{u b}^{*}+V_{c d} V_{c b}^{*}+V_{t d} V_{t b}^{*} & =0 .
\end{aligned}
$$

Each of these three relations requires the sum of three complex quantities to be zero and this can be visualised as a triangle in the complex plane. Such triangles are known as "unitarity triangles", shown in Figure 2.1.

The area of each triangle is predicted to be the same and is a measure of the $C P$ asymmetry. In $B A B A R$ the most important equation, i.e. that relevant to the $B$ meson system, is Eq. (2.9). The angles of the corresponding triangle can be measured experimentally. The quantity $\sin 2 \beta$, where $\beta \equiv \arg \left[-V_{c d} V_{c b}^{*} / V_{t d} V_{t b}^{*}\right]$ is an angle in the unitarity triangle, was the first $C P$ violating quantity to be measured at BABAR. Currently measurements of $\sin 2 \alpha$, where $\alpha \equiv \arg \left[-V_{t d} V_{t b}^{*} / V_{u d} V_{u b}^{*}\right]$, are underway. If the angles of the triangle do 


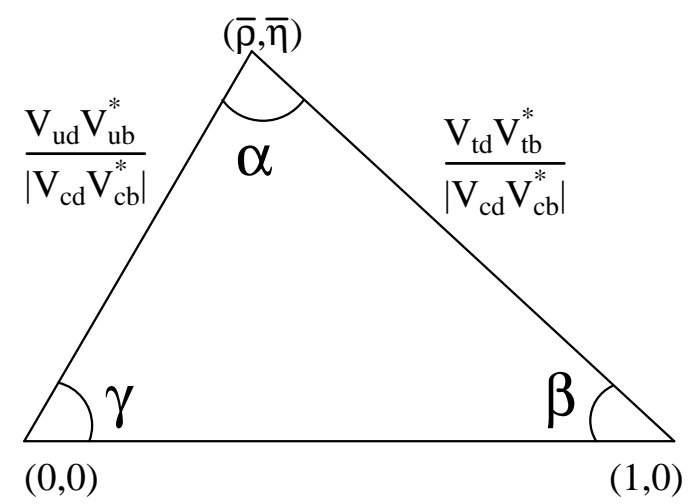

Figure 2.1: The rescaled unitarity triangle. All sides are divided by $\left|V_{c d} V_{c b}^{*}\right|$.

not sum to $\pi$ then there is evidence for physics beyond the Standard Model.

Further details about the main goals of the BABAR experiment, including the $C P$ physics goals, can be found in [52].

\subsection{Theoretical framework of $\tau$ decays}

\subsubsection{Hadronic $\tau$ decays}

Analogous to leptonic $\tau$ decays, the invariant mass amplitude for hadronic $\tau$ decays to a hadronic final state, $h$, whose initial quarks are labelled $i, j$, can be written in the form of a current-current interaction [53],

$$
\mathcal{M}\left(\tau^{-} \rightarrow h^{-} \nu_{\tau}\right)=\frac{G_{F}}{\sqrt{2}}\left|V_{i j}\right| L_{\mu} H^{\mu}
$$

where $G_{F}$ is the Fermi constant, $L_{\mu}$ is the leptonic current and $H_{\mu}$ is the hadronic current. An example of such a decay is $\tau^{-} \rightarrow \bar{K}^{0} \pi^{-} \nu_{\tau}$ whose Feynman diagram is shown in Figure 2.2. The leptonic current, with Dirac spinors $\bar{v}_{\nu_{\tau}}$ and $u_{\tau}$, is given by

$$
L_{\mu}=\bar{v}_{\nu_{\tau}} \gamma_{\mu}\left(1-\gamma_{5}\right) u_{\tau}
$$


and the hadronic current by

$$
H_{\mu}=\left\langle h^{-}\left|\left\{V_{\mu}(0)-A_{\mu}(0)\right\}\right| 0\right\rangle .
$$

In Eq. (2.12) the physical vacuum is denoted by 0. Hadronic final states are therefore created out of the QCD vacuum by the weak charged current.

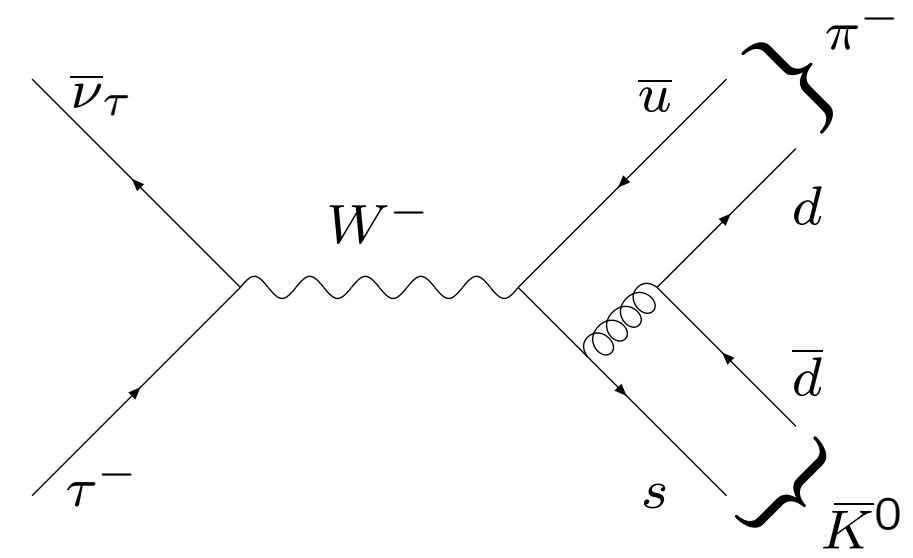

Figure 2.2: Feynman diagram of $\tau^{-} \rightarrow \bar{K}^{0} \pi^{-} \nu_{\tau}$ decay. The decay is Cabibbo suppressed.

The differential decay width can be written generally as

$$
\mathrm{d} \Gamma\left(\tau^{-} \rightarrow h^{-} \nu_{\tau}\right)=\frac{G_{F}^{2}}{4 m_{\tau}}\left|V_{i j}\right|^{2} L_{\mu \nu} H^{\mu \nu} \mathrm{d}(\text { LIPS })
$$

and can be related to the $\tau$ structure functions [54]. In Eq. (2.13), LIPS is a Lorentz invariant phase space element and the current tensors follow from squaring the invariant mass amplitude, $\mathcal{M}$, in the calculation. Using Eq. (2.13) it is possible to estimate the branching ratio $\mathcal{B}\left(\tau^{-} \rightarrow(\text { strange })^{-} \nu_{\tau}\right)$ quite simply within the framework of the Standard Model since a number of factors appear in both numerator and denominator, and therefore cancel in the branching ratio. The branching ratio $\mathcal{B}\left(\tau^{-} \rightarrow(\text { strange })^{-} \nu_{\tau}\right)$ is defined by:

$$
\mathcal{B}\left(\tau^{-} \rightarrow(\text { strange })^{-} \nu_{\tau}\right) \equiv \frac{\Gamma\left[\left(\tau^{-} \rightarrow(\text { strange })^{-} \nu_{\tau}\right)\right]}{\Gamma\left[\left(\tau^{-} \rightarrow(\text { anything })^{-} \nu_{\tau}\right)\right]} .
$$

There are four possibilities for the decay of the $\tau$ : two involve hadronic final states, i.e. $u d$ and $u s$, and two involve leptonic final states, i.e. $e$ and $\mu$. Since quarks can mix in the Standard Model, a factor of $\left|V_{u d}\right|^{2} \simeq \cos ^{2} \theta_{C} \simeq 0.95$ is introduced to accompany the 
hadronic current in the $u d$ case, and similarly a factor $\left|V_{u s}\right|^{2} \simeq \sin ^{2} \theta_{C} \simeq 0.05$ is present for the $u s$ case, where $\left|V_{u d}\right|$ and $\left|V_{u s}\right|$ are absolute CKM mixing elements and $\theta_{C}$ is the Cabibbo angle. For the leptonic current there is no mixing and so the equivalent "factor" is 1. Using Eq. (2.13), Eq. (2.14) can be recast in terms of these factors which, after some cancellation, yields:

$$
\begin{aligned}
\mathcal{B}\left(\tau^{-} \rightarrow(\text { strange })^{-} \nu_{\tau}\right) & \simeq \frac{3\left|V_{u s}\right|^{2}}{3\left|V_{u d}\right|^{2}+3\left|V_{u s}\right|^{2}+1+1} \\
& \simeq \frac{3 \sin ^{2} \theta_{C}}{5} \\
& \simeq 3 \%,
\end{aligned}
$$

where the 3 in front of the $\left|V_{i j}\right|^{2}$ factors correspond to the 3 possible quark colours. The estimated value of $3 \%$ agrees well with the experimental measurement, Table 1.2. This simple parton level description has to be refined in view of corrections such as final state interactions (hadronisation) of the quarks.

Theoretically, the hadronic $\tau$ decay width can be parameterized in terms of the spectral functions, $\rho(s)$ [55]. Experimentally, the spectral functions can be determined by measuring the invariant mass spectra of the hadronic final states, normalising them to their respective branching ratios and using the appropriate kinematic factor. The spectral functions are therefore obtained, for $J=1$ hadronic final states, using [20]

$$
\begin{aligned}
\rho_{V / A ; i j}^{(1)}(s) & =\frac{m_{\tau}^{2}}{6\left|V_{i j}\right|^{2} S_{\mathrm{EW}}} \frac{\mathcal{B}\left(\tau^{-} \rightarrow V^{-} / A^{-}(J=1)\right) \nu_{\tau}}{\mathcal{B}\left(\tau^{-} \rightarrow e^{-} \bar{\nu}_{e} \nu_{\tau}\right)} \\
& \times \frac{1}{N_{V / A ; i j}} \frac{\mathrm{d} N_{V / A ; i j}}{\mathrm{~d} s}\left[\left(1-\frac{s}{m_{\tau}^{2}}\right)^{2}\left(1+2 \frac{s}{m_{\tau}^{2}}\right)\right]^{-1}
\end{aligned}
$$

and, for $J=0$ hadronic final states, using

$$
\begin{aligned}
\rho_{V / A ; i j}^{(0)}(s) & =\frac{m_{\tau}^{2}}{6\left|V_{i j}\right|^{2} S_{\mathrm{EW}}} \frac{\mathcal{B}\left(\tau^{-} \rightarrow V^{-} / A^{-}(J=0)\right) \nu_{\tau}}{\mathcal{B}\left(\tau^{-} \rightarrow e^{-} \bar{\nu}_{e} \nu_{\tau}\right)} \\
& \times \frac{1}{N_{V / A ; i j}} \frac{\mathrm{d} N_{V / A ; i j}}{\mathrm{~d} s}\left(1-\frac{s}{m_{\tau}^{2}}\right)^{-2} .
\end{aligned}
$$

For each $\rho(s)$, the subscripts $V$ and $A$ are used to denote the vector and axial-vector spectral functions respectively and the superscripts give the spin $J$; transverse contributions are given by the $J=1$ spectral functions and longitudinal contributions by the 
$J=0$. As usual the absolute flavour $i j$ CKM matrix element is given by $\left|V_{i j}\right|$, and $S_{\mathrm{EW}}$ is an electroweak radiative correction. The quantity $\left(1 / N_{V / A ; i j}\right)\left(\mathrm{d} N_{V / A ; i j} / \mathrm{d} s\right)$ is the normalised invariant mass-squared distribution of the corresponding vector or axial-vector decay channels, and this can be measured using hadronic $\tau$ decay data. For example, ALEPH have made measurements of the invariant mass distributions using a number of $\tau$ decay channels involving strange hadronic final states and translated these measurements into the total strange spectral function in [20]. BABAR has begun similar analyses, of which the work presented in Chapter 6 is part.

Integrating Eq. (2.13) yields an expression for the $\tau$ hadronic decay rate in terms of the spectral functions $[55,56]$ :

$$
\begin{aligned}
& \Gamma\left(\tau^{-} \rightarrow h^{-} \nu_{\tau}\right)= \\
& \quad \frac{G_{F}^{2} m_{\tau}^{3}}{32 \pi^{2}} \int_{0}^{m_{\tau}^{2}} \mathrm{~d} s\left(1-\frac{s}{m_{\tau}^{2}}\right)^{2}\left\{\cos ^{2} \theta_{C}\left[\left(1+2 \frac{s}{m_{\tau}^{2}}\right)\left[\rho_{V ; u d}^{(1)}(s)+\rho_{A ; u d}^{(1)}(s)\right]+\rho_{A ; u d}^{(0)}(s)\right]\right. \\
& \left.\quad+\sin ^{2} \theta_{C}\left[\left(1+2 \frac{s}{m_{\tau}^{2}}\right)\left[\rho_{V ; u s}^{(1)}(s)+\rho_{A ; u s}^{(1)}(s)\right]+\rho_{V ; u s}^{(0)}(s)+\rho_{A ; u s}^{(0)}(s)\right]\right\} .
\end{aligned}
$$

Relative to non-strange ( $u d$ ) currents, strange (us) currents of $\tau$ decays are, from Eq. (2.18), suppressed by an amount $\left(\left|V_{u s}\right| /\left|V_{u d}\right|\right)^{2} \simeq \tan ^{2} \theta_{C}$.

The $K \pi$ spectral function is dominated by the $K^{*}(892)$ resonance, but smaller contributions from higher mass $K^{*}$ resonances have been observed [20]. Two parameterizations that have been used in practice to model wide hadronic resonances, for example the $\tau^{-} \rightarrow \rho^{-} \nu_{\tau}$ hadronic current, are:

$\diamond$ The Kühn-Santamaria model (KS) which is essentially a Breit-Wigner with energy dependent width [57].

$\diamond$ The Gounaris-Sakurai model (GS) which incorporates analyticity through finite width corrections [58].

At $B A B A R$ studies are underway which use these parameterizations in the non-strange 
sector $[59,60]$. These parameterizations should lend themselves to the $K \pi$ system too, although there are other ways to study the spectral distribution which might be better in the $K \pi$ case. For instance, for the $K_{0}^{*}(1430)^{-}$, which decays essentially entirely (or perhaps entirely, with no qualification) to $K \pi$, an $s$-dependent width relevant to the twobody $K \pi s$-wave (rather than $p$-wave) decay should be reasonable. However, in $K \pi$ scattering experiments such as LASS a non-trivial background, or indeed real structure, in the $K_{0}^{*}(1430)^{-}$region was observed [61-63]. This is likely to be true in the $\tau$ decay case too.

\subsubsection{Properties of the weak charged current}

$G$-parity is the quantum number corresponding to the conserved vector and axial-vector currents. The operation comprises isospin rotation followed by charge conjugation. Table 2.2 shows the properties of the weak current. In the non-strange sector, where $G$-parity is conserved, the properties given in Table 2.2 assert that a decaying $\tau$ lepton to a number of pions, each with $G=-1$, will proceed via the vector current if the number of pions is even or via the axial-vector if the number of pions is odd.

Conserved vector current (CVC) is the term used to describe the hypothesis that the weak current and its conjugate, together with the electromagnetic current, form an isospin triplet of conserved currents. For even $G$-parity final states only the vector current contributes. $\mathrm{CVC}$ therefore connects the decay rate to the cross-section for $e^{+} e^{-} \rightarrow$ hadrons.

In the case of strange hadronic final states it is not acceptable to neglect the strange quark mass, $m_{s}$, as can be done for the smaller $u$ and $d$ masses manifest in non-strange hadronic states. This means that it is possible to break chiral symmetry in the strange sector and therefore $G$-parity cannot be defined for strange hadronic states. As a consequence it is difficult to separate vector and axial-vector contributions in a manner analogous to the non-strange sector. CVC may be violated for decays with strange hadronic final states, i.e. the vector current is not necessarily conserved. 


\begin{tabular}{|c||c|c|}
\hline Property & Vector current & Axial-vector current \\
\hline \hline$I$ & 1 & 1 \\
\hline$G$ & +1 & -1 \\
\hline$J^{P}$ & $0^{+}$(strange), $1^{-}$ & $0^{-}, 1^{+}$ \\
\hline
\end{tabular}

Table 2.2: Properties of the weak current allowed particle final states: isospin $(I), G$-parity $(G)$ and spin-parity $\left(J^{P}\right)$. The vector current can produce scalar $\left(0^{+}\right)$and vector $\left(1^{-}\right)$hadronic final states, whilst the axial-vector current can produce pseudoscalar $\left(0^{-}\right)$and axial-vector $\left(1^{+}\right)$hadronic final states. If conserved vector current $(\mathrm{CVC})$ is obeyed, which is true for non-strange final states, the scalar $\left(0^{+}\right)$final state is not possible; the decay products of vector current interactions are always vectors $\left(1^{-}\right)$themselves. CVC is not obeyed for strange decays so the final states can be scalar $\left(0^{+}\right)$or vector $\left(1^{-}\right)$. G-parity is not a good symmetry in the case of strange final states.

\subsubsection{Operator product expansion and analyticity}

The operator product expansion (OPE) is used in a number of QCD calculations and is given by [64]

$$
i \int \mathrm{d} x e^{i q x} A(x) B(0) \simeq \sum_{k} C_{k}(q) \mathcal{O}_{k} .
$$

Eq. (2.19) represents the product $A B$ of the two local operators as a linear combination of the local operators $\mathcal{O}_{k}$ with coefficient functions $C_{k}(q)$, where $q$ is the total fourmomentum transfer of the system. Treating the OPE as an expansion in inverse powers of an energy-squared variable, $s$, the singularities of the product arise in the coefficients which are ordered according to the increasing exponent $k$ in $s^{-k}$, where $s=q^{2}=-Q^{2}$ is the usual Mandelstam variable [41].

In local quantum field theory, the product $A_{1}\left(x^{1}\right) \cdots A_{a}\left(x^{a}\right)$ of two (or several) field operators is singular for coinciding arguments. Of essential importance is the ability to define it in a neighbourhood. To this end, the operator product can be expanded in the form [65]

$$
A_{1}\left(x+x^{1}\right) \cdots A_{a}\left(x+x^{a}\right)=\sum_{k=0}^{n} f_{k}\left(x^{1}, \ldots, x^{a}\right) \mathcal{O}_{k}(x)+R_{n}\left(x, x^{1}, \ldots, x^{a}\right),
$$

where the remainder $R_{n}$ disappears in the limit $x^{j} \rightarrow 0$ as the functions $f_{k}$ become sin- 
gular, i.e. non-vanishing. This can then be generalised by assuming that any operator product can be represented as a series,

$$
A_{1}\left(x+x^{1}\right) \cdots A_{a}\left(x+x^{a}\right)=\sum_{k=0}^{\infty} f_{k}\left(x^{1}, \ldots, x^{a}\right) \mathcal{O}_{k}(x),
$$

which is asymptotic. This means that for every $N$ there exists a $k(N)$ such that the coefficients $f_{k}\left(\rho \lambda^{1}, \ldots, \rho \lambda^{a}\right)$ vanish faster than $\rho^{N}$ for all $k \geqslant k(N)$.

The OPE can be formulated within perturbation theory [66]. From this, conditions under which the OPE gives complete information on the short distance behaviour of the operator products can be found. In QCD, however, there is a strong non-perturbative component. To apply the OPE in QCD there is the problem of extending it to the non-perturbative regime. This issue has been faced since the development of the QCD sum rules [67]. For this purpose the OPE is combined with analyticity (regularity) and other non-perturbative aspects of QCD. Analyticity is used to replace the low energy integral by that along a circular contour of a sufficiently large radius in the complex s-plane [44]. This is necessary since Feynman diagrams, through which observables (including those describing high energy effects) are expressed, contain integration over small momenta where the validity of the OPE is expected to breakdown. An example of the success of this application is manifest in the finite energy sum rules (FESRs) and their relation to the hadronic $\tau$ decay spectral functions.

\subsection{4 $\tau$ hadronic decay rate ratio, $R_{\tau}$}

The ratio of the hadronic $\tau$ decay rate through the flavour, $f=i j=u d, u s$, vector $(V)$ and axial-vector $(A)$ current to the electronic decay rate is defined by [68]

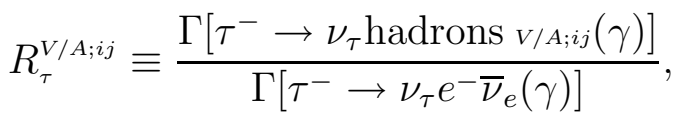

where $\gamma$ is used to indicate the presence of additional photons or lepton pairs. To first approximation $R_{\tau} \sim 3$, reflecting the fact that there are three possible quark colours. 
However, the hadronic decays are affected by radiative corrections of the strong interaction, for example virtual gluon exchange. Such corrections increase $R_{\tau}$ and scale with $\alpha_{S}$. Measuring deviations of $R_{\tau}$ from 3 therefore provides a way of measuring $\alpha_{S}$ itself.

Theoretically, the total hadronic width can be accurately calculated using the OPE along with analyticity. $R_{\tau}$ can then be expressed as $[68,69]$

$$
R_{\tau}=N_{C} S_{\mathrm{EW}}\left\{\left(\left|V_{u d}\right|^{2}+\left|V_{u s}\right|^{2}\right)\left[1+\delta^{(0)}\right]+\sum_{D \geqslant 2}\left[\left|V_{u d}\right|^{2} \delta_{u d}^{(D)}+\left|V_{u s}\right|^{2} \delta_{u s}^{(D)}\right]\right\},
$$

where $N_{C}$ is the number of quark colours (i.e. 3), $S_{\mathrm{EW}}$ is an electroweak radiative correction $\left(S_{\mathrm{EW}}=1.0201 \pm 0.0003\right)$, and $\delta_{u d}^{(D)}$ and $\delta_{u s}^{(D)}$ are corrections in the OPE dominated by terms proportional to $m_{s}^{2}$ and $m_{s}\langle q \bar{q}\rangle$ (the latter is a condensate correction). In the theoretical framework, the OPE is used to create a series of local gauge invariant operators of increasing dimension $D=2 n$ times appropriate inverse powers of $s$. A consequence of chiral symmetry is that the purely perturbative dimension-zero contribution, $\delta^{(0)}$, is identical for vector and axial-vector parts. For higher dimensions, $D \geqslant 2$, the vector and axial-vector contributions are averaged, $\delta_{i j}^{(D)} \equiv\left(\delta_{V ; i j}^{(D)}+\delta_{A ; i j}^{(D)}\right) / 2$. These averages contain implicit suppression factors of $1 / m_{\tau}^{D}$.

\subsubsection{Spectral weights and their relation to $\boldsymbol{R}_{\tau}$}

Hadrons produced in $\tau$ decays are created from the QCD vacuum via the charged weak current. This property means that all the physics is contained within the spectral functions. Moreover, the produced hadronic systems have isospin $I=1$ and spin-parity $J^{P}=0^{+}, 1^{-}$ $(V)$ and $J^{P}=0^{-}, 1^{+}(A)$. The spectral functions are directly related, normalised to their respective branching ratios and with kinematic corrections, to the invariant mass spectra of the hadronic final states. The $\tau$ hadronic decay rate ratio (Eq. (2.22)) can be written compactly in terms of weighted integrals over the spin $J=0$ and $J=1$ hadronic spectral functions [2].

From Eq. (2.18), an expression involving $R_{\tau}$ can be obtained by dividing through by the 
electronic decay width, numerical factors and CKM matrix elements: [2, 49]:

$$
\begin{aligned}
\frac{R_{\tau}^{V / A ; i j}}{\left[12 \pi^{2}\left|V_{i j}\right|^{2} S_{\mathrm{EW}}\right]} & =\int_{0}^{1} \mathrm{~d} y_{\tau}\left(1-y_{\tau}\right)^{2}\left[\left(1+2 y_{\tau}\right) \rho_{V / A ; i j}^{(0+1)}(s)-2 y_{\tau} \rho_{V / A ; i j}^{(0)}(s)\right] \\
& =\frac{-1}{2 \pi i} \oint_{\left|y_{\tau}\right|=1} \mathrm{~d} y_{\tau}\left(1-y_{\tau}\right)^{2}\left[\left(1+2 y_{\tau}\right) \Pi_{V / A ; i j}^{(0+1)}(s)-2 y_{\tau} \Pi_{V / A ; i j}^{(0)}(s)\right] \\
& \equiv\left[12 \pi^{2}\left|V_{i j}\right|^{2} S_{\mathrm{EW}}\right]^{-1} \int_{0}^{m_{\tau}^{2}} \mathrm{~d} s \frac{\mathrm{d} R_{\tau}^{V / A ; i j}(s)}{\mathrm{d} s},
\end{aligned}
$$

where $y_{\tau}=s / m_{\tau}^{2},\left|V_{i j}\right|$ is the CKM flavour $i j$ matrix element, $S_{\mathrm{EW}}$ is an electroweak correction, and $\rho_{V / A ; i j}^{(J)}(s)$ is the spectral function of the corresponding spin $J$ part of the flavour $i j V / A$ correlator, $\Pi_{V / A ; i j}^{(J)}(s)^{2}$. Figure 2.3 shows the integration contour of interest. The contour integral appearing in Eq. (2.24) follows from applying analyticity and the OPE as done by Pich [70] to measure the $\tau$ decay rate to determine the QCD running coupling constant, $\alpha_{S}$, at the $m_{\tau}$ scale. The spectral functions themselves are defined by

$$
\rho_{V / A ; i j}^{(J)}\left(q^{2}\right)=\frac{1}{\pi} \operatorname{Im} \prod_{V / A ; i j}^{(J)}\left(q^{2}\right),
$$

where $q$ is the 4-momentum of the hadronic system, i.e. $q^{2}$ corresponds to the invariant mass squared of the final state hadrons $\left(q^{2}=s\right)$.

Theoretical analysis of the hadronic $\tau$ decay width involves the two-point correlation functions associated with the time-ordered vector, $V_{i j}^{\mu}(x) \equiv \bar{q}_{j} \gamma^{\mu} q_{i}$ and axial-vector, $A_{i j}^{\mu}(x) \equiv \bar{q}_{j} \gamma^{\mu} \gamma_{5} q_{i}$, colour-singlet massless quark currents:

$$
\begin{aligned}
\Pi_{V ; i j}^{\mu \nu}\left(q^{2}\right) & \equiv i \int \mathrm{d}^{4} x e^{i q x}\left\langle 0\left|T\left\{V_{i j}^{\mu}(x) V_{i j}^{\nu}(0)^{\dagger}\right\}\right| 0\right\rangle ; \\
\Pi_{A ; i j}^{\mu \nu}\left(q^{2}\right) & \equiv i \int \mathrm{d}^{4} x e^{i q x}\left\langle 0\left|T\left\{A_{i j}^{\mu}(x) A_{i j}^{\nu}(0)^{\dagger}\right\}\right| 0\right\rangle .
\end{aligned}
$$

The correlators admit Lorentz decompositions and so can be broken up into separate longitudinal $(J=0)$ and transverse $(J=1)$ contributions:

$$
\begin{aligned}
\Pi_{V / A ; i j}^{\mu \nu}\left(q^{2}\right) & \equiv i \int \mathrm{d}^{4} x e^{i q x}\left\langle 0\left|T\left\{J_{V / A}^{\mu}(x) J_{V / A}^{\nu}(0)^{\dagger}\right\}\right| 0\right\rangle \\
& =\left(q^{\mu} q^{\nu}-q^{2} g^{\mu \nu}\right) \Pi_{V / A}^{(1)}\left(q^{2}\right)+q^{\mu} q^{\nu} \Pi_{V / A}^{(0)}\left(q^{2}\right),
\end{aligned}
$$

\footnotetext{
${ }^{2}$ Providing there are no kinematic singularities, the combination $\rho_{V / A ; i j}^{(0+1)}(s) \equiv \rho_{V / A ; i j}^{(0)}(s)+\rho_{V / A ; i j}^{(1)}(s)$ and $s \rho_{V / A ; i j}^{(0)}(s)$ correspond to the scalar correlators $\Pi_{V / A ; i j}^{(0+1)}(s) \equiv \Pi_{V / A ; i j}^{(0)}(s)+\Pi_{V / A ; i j}^{(1)}(s)$ and $s \Pi_{V / A ; i j}^{(0)}(s)$.
} 
where $J_{V / A}^{\mu}$ can be either the $V$ or $A$ current. For a given channel, the experimental decay distribution can be used to extract the kinematically-weighted linear combination of $J=(0+1)+(0)$ spectral functions.

Non-strange $(u d)$ hadronic $\tau$ decay data provide very detailed information on the sum $\rho_{V}^{(0+1)}(s)+\rho_{A}^{(0+1)}(s)$. For states containing only pions, $G$-parity allows unambiguous separation of the $V$ and $A$ components of this sum. Consequently the separate $V$ and $A$ terms as well as the difference $\Delta \rho \equiv \rho_{V}^{(0+1)}(s)-\rho_{A}^{(0+1)}(s)$ are known very accurately from experiment. A recent theoretical treatment of the non-strange $(u d)$ spectral functions is given in [71]. In the strange (us) sector things are not as simple since there are longitudinal contributions to hadronic $\tau$ decay via the vector current (Table 2.2). As a result CVC cannot be assumed to hold and $G$-parity cannot be invoked. Therefore, the ability to separate $V$ and $A$ components experimentally in the strange sector is much more difficult and as yet has not been done nor has a suitable method for doing so been presented.

The fact that vector and axial-vector currents are not conserved in $\tau$ decays with strange final states is one way of explaining why a massive vector, such as the $W$, can couple to spin 0 as well as spin 1 particles, i.e. that there are longitudinal contributions to $\tau$ decays to strange hadronic final states. In this description the inclusive sum over final states of a given total $s$ corresponds to the imaginary part of the relevant current-current correlator (i.e. the relevant spectral function). For non-strange $(u d)$ final states the vector current is conserved (CVC) since $\left(m_{u}-m_{d}\right)$ is so small it can be neglected. If the current is not conserved, which is the case for the strange (us) vector and strange axial-vector currents due to $\left(m_{u}-m_{s}\right)$ and $\left(m_{u}+m_{s}\right)$ being non-zero respectively, then the correlator has a part proportional to $\left(q^{\mu} q^{\nu}-q^{2} g^{\mu \nu}\right)$ for spin 1 , and a non-zero part proportional to $q^{\mu} q^{\nu}$ for spin 0 which arises from the coupling of each of $J^{\mu}$ and $J^{\nu}$ to a scalar state, which has to have a factor of momentum in the coupling to carry the Lorentz index. These terms can be clearly seen in Eq. (2.28). For the axial-vector channel, $\rho_{A ; i j}^{(0)}(s)$ is saturated by the flavour $i j$ Goldstone boson pole in the chiral limit and has non-Goldstone contributions proportional to $\left(m_{i}+m_{j}\right)^{2}$ [2]. Therefore, apart from $\rho_{A ; u d}^{(0)}(s)$, the $\pi$-pole contribution, 
all other $\rho_{A ; i j}^{(0)}(s)$ can be neglected. For the vector channel, $\rho_{V ; i j}^{(0)}(s)$ is proportional to $\left(m_{i}-m_{j}\right)^{2}$. Although $\rho_{V ; u d}^{(0)}$ can be safely neglected, $\rho_{V ; u s}^{(0)}$, the contribution at the $K$-pole, due to the relatively large strange quark mass, cannot. If the currents had been conserved then there would only be the spin 1 part of the correlator/spectral function, but when they are not conserved, which is borne out in practice for strange hadronic final states, then both spin 1 and spin 0 occur. The spin 0 part is, however, chirally-suppressed due to the factors of quark masses. These mass factors measure the degree of non-conservation and go some way to explaining why the expected branching ratio is small.

Cauchy's theorem, along with analyticity, implies that the correlators (П) that have no kinematic singularities satisfy finite energy sum rules (FESRs). The contour integral appearing in Eq. (2.24) therefore follows as a direct consequence of the general FESR relation, which for weight functions $w(s)$ analytic in the region $|s|<S$ of the complex $s$-plane and for any $s_{0}<S$ have the form [2]

$$
\int_{0}^{s_{0}} \mathrm{~d} s \rho(s) w(s)=\frac{-1}{2 \pi i} \oint_{|s|=s_{0}} \mathrm{~d} s \Pi(s) w(s) .
$$

In the case of Eq. (2.24), which is expressed in terms of $y_{\tau}$, the contour integral is along a circle of radius 1. Equivalently, in the complex $s$-plane the radius of the contour is $m_{\tau}^{2}$, as shown in Figure 2.3. If $s_{0}$ is sufficiently large in order that the correlators (П) may be approximated by their OPEs, i.e. so that contributions from non-perturbative effects confining quarks in hadrons are small, which is true as $s_{0} \rightarrow m_{\tau}^{2}$, there exists a relationship between spectral data and the OPE parameters. This assumption was made in formulating the contour integral for $\operatorname{Im} \Pi$ in the complex $s$-plane in Eq. (2.24). The analyticity property of the correlator $\Pi^{(J)}$ allows the evaluation of Eq. (2.24) since $\Pi^{(J)}$ is analytic in the complex $s$-plane everywhere except on the positive real axis, where singularities exist.

Experimental data provides access to the $u d$-us spectral difference and hence to integrals of the corresponding correlator difference. In other words, the LHS of Eq. (2.29) can be determined using the decay constants of the relevant scalar and pseudoscalar resonances, while the RHS can be evaluated using the OPE providing $s_{0}$ is large enough. At such 


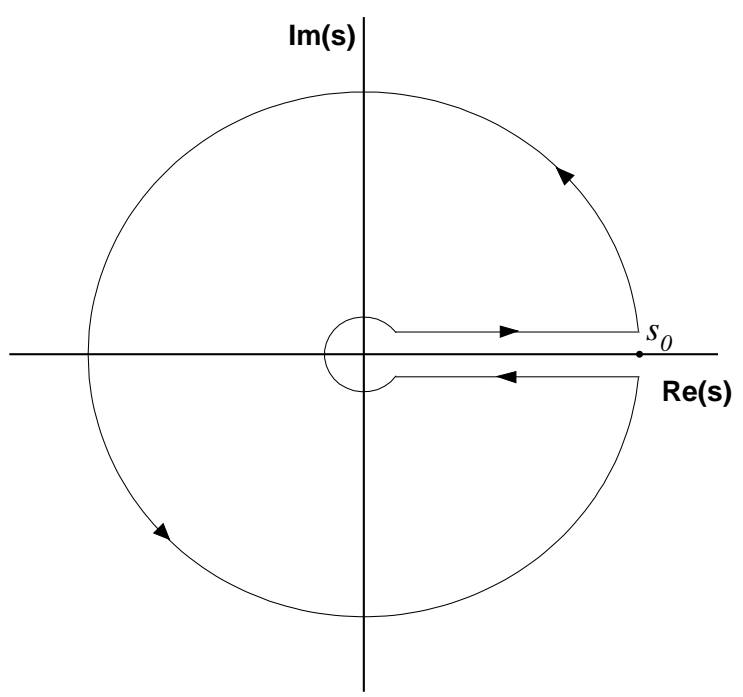

Figure 2.3: Integration contour in the complex s-plane. Analyticity is used to replace an integral along the cut by that along a circle of radius $m_{\tau}^{2}$.

large values of $s_{0}$ these differences are dominated by the $D=2$ term of the OPE which is proportional to $m_{s}^{2}$.

A modified version of Eq. (2.24) is obtained by multiplying the integrands of both the spectral and OPE representations of $R_{\tau}^{V / A ; i j}$ by the factor $\left(1-y_{\tau}\right)^{k} y_{\tau}^{l}$. This representation is called "the $(k, l)$ spectral weight sum rule". Such weighted integrals are known as moments. The advantage of recasting Eq. (2.24) in this manner is that a spin separation of the $i j=u s$ spectral data is no longer needed in order to evaluate the integral involving the spectral functions, i.e. an experiment need not undertake the spin separation so that the spectral side can be equated with the OPE side of the FESR relation (Eq. (2.29)). As mentioned before, because there is no such concept as $G$-parity for strange final states, separate $V$ and $A$ measurements are tough to make experimentally; applying the spectral weights therefore negates this necessity.

For any $s_{0} \leqslant m_{\tau}^{2}$ therefore, hadronic $\tau$ decay data can be used to evaluate the spectral integrals required for various $V$ and $A$ FESRs. $\alpha_{S}$ and $m_{s}$, which appear on the OPE side of the FESR relation, can be evaluated using spectral integrals providing that there 
exists a window of $s_{0}$ below $m_{\tau}^{2}$ such that the breakdown of the OPE representation of the relevant correlator ("duality violation") is negligible. Figure $2.4 a$ shows how the $(0,0)$ weighted spectral integrals vary for $s_{0} \leqslant m_{\tau}^{2}$. Figure $2.4 b$ shows the same thing for the $w_{10}$ weight, which was constructed by Maltman and Kambor [35] to have better convergence behaviour. Figure $2.4 a$ and Figure $2.4 b$ are made using the values from Table A.2. The origin of these values is described in Appendix A.
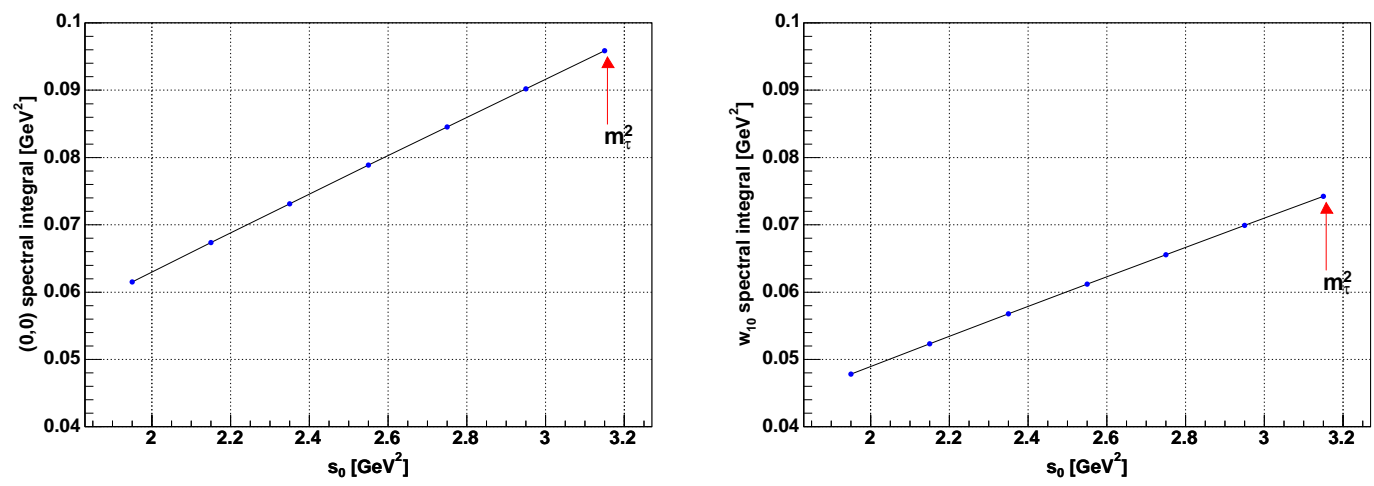

Figure 2.4: ud spectral integrals for different $s_{0}$ for the weight cases a) $(0,0)$ (left) and $\boldsymbol{b}) w_{10}$ (right). When $s_{0}=m_{\tau}^{2}$ this is the $R_{\tau}$ integral, up to constant factors.

\subsubsection{OPE regime of validity for hadronic $\tau$ decay data}

In order to use hadronic $\tau$ decay data to determine parameters appearing in the OPE it is crucial to verify that some of the scales kinematically accessible in $\tau$ decay lie in a region of validity of the OPE:

$\diamond$ For very large $s_{0}$ the OPE is expected to provide a reliable representation of hadronic correlators over the whole circle $|s|=s_{0}$ in the complex $s$-plane. The corresponding spectral function, $\rho\left(s_{0}\right)$, will also be well represented at large $s_{0}$. This is sometimes referred to as the regime of validity of "local duality" (LD) [2].

$\diamond$ Moving towards lower $s_{0}$, the OPE is expected to first show signs of a breakdown 
for those $s$ on the circle $|s|=s_{0}$ near the positive real axis because singularities exist on the positive real axis [72]. It is therefore thought that the OPE, while not reliable near the positive real axis, is reliable over most of the rest of the circle. The regime of "intermediate" scales for $s_{0}$ for which this is the case is sometimes referred to as the regime of validity of "semi-local duality" (SLD).

$\diamond$ At small $s_{0}$, the OPE is expected to be unreliable over the whole circle $|s|=s_{0}$. This is sometimes referred to as "duality violation".

Even though strong duality violations exist for the $s^{k}$-weighted FESRs for the $u d$ correlator, the FESR for $R_{\tau}^{V ; u d}$ (Eq. (2.24)) is in close agreement with experiment $[15,17] . R_{\tau}^{V ; u d}$ has the kinematic weight $\left(1-y_{\tau}\right)^{2}\left(1+2 y_{\tau}\right)$ and is actually a linear combination of four $s^{k}$-weighted FESRs. So, an obvious question is: given duality violations can exist, why in the case of $R_{\tau}^{V ; u d}$ should there be such close agreement between the FESR and the experimental data? The key to answering this question is that at the point $s=m_{\tau}^{2}$, where the circle $|s|=m_{\tau}^{2}$ crosses the positive real axis, the hadronic phase space comes to an end. Consequently the kinematic weight $\left(1-y_{\tau}\right)^{2}\left(1+2 y_{\tau}\right)$ has a double zero at $|s|=m_{\tau}^{2}$. OPE contributions from the part of the integration region near the positive real axis, where the validity of the OPE is expected to be in jeopardy, are therefore suppressed by this double zero. It is precisely this feature that is thought to explain the success of the $R_{\tau}^{V ; u d}$ FESR.

In a similar manner, other FESRs with suppressions of the OPE contributions from this region are also expected to be well satisfied, i.e. by localising the breakdown of the OPE to the vicinity of the positive real axis for scales $s_{0} \simeq m_{\tau}^{2}$.

\subsubsection{Determination of $\alpha_{S}$}

The QCD analysis of the non-strange inclusive $\tau$ decay width has led to precise measurements of the strong coupling constant at the $\tau$ mass scale, $\alpha_{S}\left(m_{\tau}^{2}\right)$. Using the FESR relation (Eq. (2.29)) the QCD phenomena of a $\tau$ with $s_{0}<m_{\tau}^{2}$ can be investigated. 
Theoretically, correlators that show a reduced level of duality violation are chosen as a starting point. This helps reduce the theoretical systematic error associated with such duality violation which is a difficult uncertainty to assess. A suitable correlator happens to come from the $u d V+\mathrm{A}$ combination. Another reason for this choice is to reduce the significant uncertainty associated with the gluon condensate which dominates the nonperturbative corrections on the OPE side. As it happens, weighting so as to suppress the $D=4$ term achieves this.

In Maltman's $\alpha_{S}$ extraction [2] these techniques are put into practice. $\alpha_{S}$ is extracted using the weighted FESR ${ }^{3}$ at $s_{0} \simeq m_{\tau}^{2}$, i.e. weighted with the factor $\left(1-y_{\tau}\right)^{2}\left(1+2 y_{\tau}\right)$. A weight with a double zero and the highest possible value for $s_{0}$ are chosen to increase the chance that duality violation effects are insignificant. The $D=8 \mathrm{OPE}$ contribution is not suppressed by any factor of $\alpha_{S}$ for the chosen weight. It does however scale as $s_{0}^{-3}$ which, since a high value of $s_{0}$ has been chosen, seems safe to neglect. The spectral integrals are used as the experimental input, which in the case of [2] come from the ALEPH measurements. In Maltman's analysis, the value for $\alpha_{S}$ obtained for the $D=0$ contribution is:

$$
\alpha_{S}\left(m_{\tau}\right)=0.345 \pm 0.026
$$

where the quoted uncertainty includes the quadrature combination of theoretical and experimental uncertainties. In practice, however, the ALEPH central value, that is considered both experimentally and theoretically robust, is currently the most common input used by theorists to a number of QCD calculations including the extraction of $m_{s}$ and the determination of $\left|V_{u s}\right|$. This value is [17]:

$$
\alpha_{S}\left(m_{\tau}\right)=0.334 \pm 0.022
$$

and the uncertainty is dominated by theory.

\footnotetext{
${ }^{3}$ Maltman actually uses "pinched"FESRs (pFESRs), but that is a technicality left to the reference [2].
} 


\subsubsection{Extraction of the strange quark mass, $m_{s}$}

The strange mass determination from hadronic $\tau$ decays depends sensitively on $\left|V_{u s}\right|$. Sensitivity to the strange quark mass can be enhanced by considering an $S U(3)_{f}$ flavour breaking difference. Defining $\Delta R_{\tau}^{i j} \equiv R_{\tau}^{V ; i j}+R_{\tau}^{A ; i j}$ it is clear that

$$
\Delta R_{\tau} \equiv \frac{R_{\tau}^{V / A ; u d}}{\left|V_{u d}\right|^{2}}-\frac{R_{\tau}^{V / A ; u s}}{\left|V_{u s}\right|^{2}}
$$

vanishes in the $S U(3)_{f}$ flavour limit. Consequently, in the difference, flavour independent uncertainties also vanish. By construction therefore, the $D=0$ (mass-independent) part of the perturbative contribution to the flavour breaking correlator difference $\Delta \prod_{V / A}^{(J)}(s) \equiv$ $\Pi_{V / A ; u d}^{(J)}(s)-\Pi_{V / A ; u s}^{(J)}(s)$ and spectral function difference $\Delta \rho_{V / A}^{(J)}(s) \equiv \rho_{V / A ; u d}^{(J)}(s)-\rho_{V / A ; u s}^{(J)}(s)$ cancels in the $S U(3)_{f}$ flavour limit. This leaves the $D=2$ term in the OPE of $\Delta \Pi_{V / A}^{(J)}(s)$ dominating which is proportional to $m_{s}^{2}$. Hadronic $\tau$ decay data can thus be used to determine $m_{s}$ by creating flavour breaking differences of $u d$ and $u s$ spectral integrals and equate these using the FESR relation (Eq. (2.29)) with the equivalent OPE contour integrals involving the unknown parameter $m_{s}$.

To extract $m_{s}$, not only must a rescaled flavour breaking difference be constructed using the decay distributions (Eq. (2.24)) by rescaling the $u d$ decay distribution by a factor $1 /\left|V_{u d}\right|^{2}$ and the us decay distribution by a factor $1 /\left|V_{u s}\right|^{2}$ as in Eq. (2.32), but also weighting must be imposed to counter the inability, at least so far, to separate the $V$ and $A$ currents experimentally. Weighted integrals of the difference of the rescaled spectral functions produce flavour breaking observables from which $m_{s}$ can be extracted $[2,21,49,73]$ :

$$
\Delta R_{\tau}^{(k, l)} \equiv \frac{R_{V+A ; u d}^{(k, l)}}{\left|V_{u d}\right|^{2}}-\frac{R_{V+A ; u s}^{(k, l)}}{\left|V_{u s}\right|^{2}},
$$

where

$$
R_{V+A ; i j}^{(k, l)} \equiv \int_{0}^{m_{\tau}^{2}} \mathrm{~d} s\left(1-y_{\tau}\right)^{k} y_{\tau}^{l} \frac{\mathrm{d} R_{V+A ; i j}(s)}{\mathrm{d} s} .
$$

In so doing, flavour independent uncertainties drop out yielding

$$
\Delta R_{\tau}^{(k, l)}=3 S_{\mathrm{EW}} \sum_{D \geqslant 2}\left(\delta_{u d}^{(k, l)(D)}-\delta_{u s}^{(k, l)(D)}\right),
$$


which results from substituting Eq. (2.23) into Eq. (2.33).

As yet, however, attempts to determine the strange quark mass using the FESR relation have been hindered by the poor convergence of the integrated OPE representation of the longitudinal contributions and the sensitivity of the $u d$-us spectral differences to the uncertainty in $\left|V_{u s}\right|^{2}$ and small changes in the measured us branching fraction. At present, the large us experimental uncertainties above the $K^{*}$ force the use of weights which in turn produce a large amount of $u d$-us cancellation, i.e. a $m_{s}$ sensitivity to $\left|V_{u s}\right|$. A recent unitarity fit for the CKM matrix gave the value $\left|V_{u s}\right|=0.2225 \pm 0.0021$, resulting in [73]

$$
m_{s}(2 \mathrm{GeV})=117 \pm 17 \mathrm{MeV} .
$$

Comparing this with the PDG central value $\left|V_{u s}\right|=0.2196 \pm 0.0026$, resulting in

$$
m_{s}(2 \mathrm{GeV})=103 \pm 17 \mathrm{MeV},
$$

shows that $m_{s}$ from the latter $\left|V_{u s}\right|$ value is about one standard deviation smaller. This highlights the extent of sensitivity of the strange quark mass measurement to $\left|V_{u s}\right|$.

With more experimental data there is expected to be a reduction in the level of high- $s$ suppressions which will allow the construction of alternative weights with a reduced level of $u d$-us cancellation. It is therefore hoped that the situation will improve with $\tau$ data from the $B$ Factory experiments, such as BABAR, where the statistics are much higher than in previous experiments.

\subsubsection{Longitudinal contributions to hadronic $\tau$ decay}

The determination of $m_{s}$ has been made using both the sum rule and lattice approaches, a summary of which is given in [10]. Currently, the most reliable sum rule approach is based on flavour breaking in hadronic $\tau$ decay [35]. The reason for this is two-fold:

$\diamond$ Spectral data from experiment is available over the whole kinematic range applicable to the sum rules. 
$\diamond$ Theoretically (OPE), flavour independent instanton and renormalisation effects that create uncertainties in analyses of the strange scalar and pseudoscalar channels cancel in forming the flavour breaking $\tau$ decay difference.

Unfortunately, the $\tau$ decay sum rules are complicated by the behaviour of the OPE representation of the longitudinal contributions to the inclusive decay rate.

Recent attempts to determine $m_{s}$ using hadronic $\tau$ decay data have been inclusive analyses based on the so-called $(k, 0)$ spectral weights from the set $(k, l)[35,73]$. However, the OPE representations of the longitudinal contributions appearing in these analyses not only suffer from the resulting poor convergence, but also have an unphysical $k$ dependence. Although this is within errors, it is disturbing. The consequence of this is a significant unphysical decrease in $m_{s}$ with increasing $k$.

Uncertainties in the experimental data are thought to give rise to this unphysical $k$ dependence and this behaviour may disappear upon improvements in experimental precision. In the past the ALEPH data has been most extensively used as input to the theoretical calculation. The lower moments are particularly sensitive to the higher energy region of the measured spectrum and so new experimental results would impact upon these. A recent analysis by CLEO [29] measured

$$
\mathcal{B}\left(\tau^{-} \rightarrow K^{-} \pi^{-} \pi^{+} \nu_{\tau}\right)=(3.84 \pm 0.14 \text { (stat) } \pm 0.38 \text { (syst) }) \times 10^{-3}
$$

The CLEO result gives a higher value for $\mathcal{B}\left(\tau^{-} \rightarrow K^{-} \pi^{-} \pi^{+} \nu_{\tau}\right)$ than the ALEPH measurement [11],

$$
\mathcal{B}\left(\tau^{-} \rightarrow K^{-} \pi^{-} \pi^{+} \nu_{\tau}\right)=(2.14 \pm 0.37 \text { (stat) } \pm 0.29 \text { (syst) }) \times 10^{-3}
$$

which has so far been the input for theoretical calculations. Theorists hope that this is corroborated by an updated OPAL measurement currently in progress. A previous OPAL result [32] gave

$$
\mathcal{B}\left(\tau^{-} \rightarrow K^{-} \pi^{-} \pi^{+} \nu_{\tau}\right)=(3.60 \pm 0.82 \text { (stat) } \pm 0.48 \text { (syst) }) \times 10^{-3}
$$


but the uncertainties are too large to assess whether it agrees better with the CLEO or ALEPH measurement. The CLEO measurement agrees better with theory and works towards removing the $k$ dependence. This is precisely why theorists hope that the updated OPAL result will be more comparable to the CLEO measurement.

In Maltman's treatment [35], additional sum rule constraints, decay constants of the excited resonances in the strange scalar and pseudoscalar channels can be determined and used to evaluate the longitudinal spectral contributions to the $(k, 0)$ sum rules. At small $k$ the uncertainties introduced by experimental input to the calculations dominates over theoretical uncertainties, but with increasing $k$ the converse is true. Here the poor convergence problem at scale $m_{\tau}^{2}$ lies with the OPE contribution involving the longitudinal contributions contained in the weighted product $w_{L}^{(k)}\left(y_{\tau}\right) \Pi_{V / A}^{(0)}(s)$. Making use of accurately known $\pi / K$-pole contributions, the longitudinal contributions can be determined with an accuracy at the few $\%$ level. As a result, longitudinal subtraction is possible which leaves just a weighted longitudinal plus transverse $(0+1)$ sum term, $w_{L+T}^{(k)}\left(y_{\tau}\right) \Pi_{V / A}^{(0+1)}(s)$, for which the OPE representation converges. For the $k=0$ case, i.e. the $(0,0)$ moment, the weight factors are those explicitly written in Eq. (2.24). More generally, for any $k$ $((k, 0)$ moments $)$ an additional weight factor of $\left(1-y_{\tau}\right)^{k}$ is used too.

Any determination of $m_{s}$ based on inclusive $(k, 0)$ spectral weight analyses of flavour breaking in hadronic $\tau$ decay have an unavoidable and unphysical dependence on $k$ resulting in an unphysical extraction of $m_{s}$. This problem has been shown to result from the unphysical behaviour with respect to $k$ of the OPE representations to the $u d$ and $u s$ correlators when truncated at $D=6$. If $D=6$ is justified, then the $D=2$ part of the OPE is to blame, the integrated contour of which is already known to be poorly converging.

In Maltman's opinion, the bad behaviour of the OPE representation of the longitudinal contributions prevents the reliable use of an inclusive analysis. Indeed, Maltman says that theorists are forced to make a theoretical evaluation of the longitudinal contributions to the spectrum. Experimentally, a measurement of the $\mathcal{B}\left(\tau^{-} \rightarrow K_{0}^{*}(1430)^{-} \nu_{\tau}\right)$ and the study of 
the spectral functions, including a partial wave separation of the $s$-wave $K_{0}^{*}(1430)^{-}$from the other $p$-wave $K^{*}$ resonances, particularly the $K^{*}(1410)^{-}$which has a branching ratio to $K \pi$ of $(6.6 \pm 1.3) \%$ [39], would be very useful: a longitudinal subtraction could be performed using experimental data ${ }^{4}$. However, the $K_{0}^{*}(1430)^{-}$has not yet been observed in hadronic $\tau$ decay data; so far there only exists a limit of $\mathcal{B}\left(\tau^{-} \rightarrow K_{0}^{*}(1430)^{-} \nu_{\tau}\right)<$ $5 \times 10^{-4}$ measured by ALEPH [20]. The ALEPH results, although in the $K^{*}$ region the uncertainties are around the 6-8\% level, above $\sim 1 \mathrm{GeV}^{2}$ they increase to around $20-30 \%[2]$.

At ALEPH, the $\mathcal{B}\left(\tau^{-} \rightarrow K_{0}^{*}(1430)^{-} \nu_{\tau}\right)$ limit was reached using a Breit-Wigner PDF to describe the $s$-wave state. A model involving a Breit-Wigner scalar contribution to the $\tau^{-} \rightarrow(K \pi)^{-} \nu_{\tau}$ vector current is described in [74]. Such a model is far from obvious; $K \pi$-scattering experiments such as LASS suggest a very different model that has a long non-trivial background, or possibly genuine structure, below the "resonance" [61-63]. E791 has also studied scalar contributions to $K \pi$ [75], and a comparison between the LASS and E791 results is discussed in [62]. What is certain is that the $s$-wave shape is not well understood and is a strong motivation for further analysis, particularly at $B$ Factory experiments where the statistics are larger. A case in point is the use of the LASS measurements to determine the $s$-wave contributions from $K \pi$-scattering [76,77]. These results are used as a handle on the longitudinal contributions in preference to those from ALEPH and used to extract $m_{s}$ and $\left|V_{u s}\right|$ in [73].

For $\mathcal{B}\left(\tau^{-} \rightarrow K_{0}^{*}(1430)^{-} \nu_{\tau}\right)$, Maltman predicts:

$$
\mathcal{B}\left(\tau^{-} \rightarrow K_{0}^{*}(1430)^{-} \nu_{\tau}\right) \sim 6 \times 10^{-5}
$$

with a conservative uncertainty of around $50 \%[2,35]$. This is approximately a factor of eight below the ALEPH upper bound. However, Maltman emphasises that even an improvement in the ALEPH limit to $\mathcal{B}\left(\tau^{-} \rightarrow K_{0}^{*}(1430)^{-} \nu_{\tau}\right)<1 \times 10^{-4}$, i.e. by a factor

\footnotetext{
${ }^{4}$ There is another $s$-wave $K^{*}$ resonance, $K_{0}^{*}(1950)^{-}$, which is approximately a factor 20 times smaller than the $K_{0}^{*}(1430)^{-}$contribution. Moreover, it is beyond the phase space limit in $\tau$ decay governed by $m_{\tau}$. It is therefore expected that in $\tau$ decay data one is well into the tails of the $K_{0}^{*}(1950)^{-}$resonance distribution and so it can be safely neglected.
} 
of five, would already be a significant improvement for theorists. In addition, following our discussion at Elba 2003 [3], Jamin has also made a preliminary calculation of $\mathcal{B}\left(\tau^{-} \rightarrow\right.$ $\left.K_{0}^{*}(1430)^{-} \nu_{\tau}\right)[78,79]$,

$$
\mathcal{B}\left(\tau^{-} \rightarrow K_{0}^{*}(1430)^{-} \nu_{\tau}\right)=(4.17 \pm 0.40) \times 10^{-4}
$$

which is more optimistic than Maltman's prediction and is close to the ALEPH limit [20]. Jamin emphasises that the $K_{0}^{*}(1430)^{-}$should be understood as a $K \pi$ system in a $s$-wave state and as such it is non-trivial to separate what is "resonance" and what is "rest" for the scalar system. The number given in Eq. (2.42) actually corresponds to the branching fraction into the $K \pi$ system in a $s$-wave state. As such it depends how the resonant and non-resonant contributions are separated. If an analysis were to really count the $s$-wave $K \pi$ system, then Eq. (2.42) should apply. However, using a parameterization of resonance plus background, Jamin expects that the number in Eq. (2.42) would be smaller and the branching fraction $\mathcal{B}\left(\tau^{-} \rightarrow K_{0}^{*}(1430)^{-} \nu_{\tau}\right)$ would depend on the particular parameterization of the spectrum. Neglecting these possible complications for now and using Eq. (2.42), Jamin estimates that measuring the corresponding rate to better than about $10 \%$ could have an important impact for the determination of the strange quark mass from scalar sum rules.

\subsubsection{Determining $\left|V_{u s}\right|$ from $R_{\tau}$}

Taking as input the strange quark mass, it is possible to determine $\left|V_{u s}\right|$ from the moments for $S U(3)_{f}$ flavour breaking differences of hadronic $\tau$ decay rates. In [73] they use the

flavour breaking $\tau$ sum rule on $\left|V_{u s}\right|$ itself. It is therefore possible to determine $\left|V_{u s}\right|$ using the same sum rule by assuming an average value for $m_{s}$ as extracted from other sources. The result they obtain is

$$
\left|V_{u s}\right|=0.2179 \pm 0.0045
$$


The uncertainty is currently dominated by the experimental measurement of $R_{\tau}$ for strange hadronic final states [11],

$$
R_{\tau}^{\text {strange }} \equiv R_{\tau}^{V+A ; u s}=0.1625 \pm 0.0066 .
$$

If it could be reduced by a factor of two, $\left|V_{u s}\right|$ from hadronic $\tau$ decays would be more precise than the current PDG value [39]. With a large enough sample of data, like that achievable at $B A B A R$, a precise measurement of the strange spectral function from hadronic $\tau$ decay data would allow a simultaneous extraction of both $\left|V_{u s}\right|$ and $m_{s}$. 


\section{3}

\section{The BABAR experiment}

\subsection{PEP-II}

\subsubsection{Overview}

Figure 3.1 shows the PEP-II linear accelerator based at SLAC. The design is described in detail in $[80,81]$. It consists of two storage rings that are injected with positrons of energy $3.1 \mathrm{GeV}$ in the low energy ring (LER) and electrons of energy $9.0 \mathrm{GeV}$ in the high energy ring (HER). The electrons and positrons collide at a centre of mass energy of $10.58 \mathrm{GeV}$, corresponding to the mass of the $\Upsilon(4 S)$ resonance. Decays from this resonance are almost all $B \bar{B}$ and so PEP-II is capable of producing many $B$ mesons. This is essential in order to study $C P$ violation in the $B$ meson system because the relevant decay channels have small, $\mathcal{O}\left(10^{-5}\right)$, branching ratios.

To achieve the necessary large data samples for such studies a high luminosity must be delivered. PEP-II has already surpassed its design luminosity of $3.0 \times 10^{33} \mathrm{~cm}^{-2} \mathrm{~s}^{-1}$ and consistently delivers over $4.0 \times 10^{33} \mathrm{~cm}^{-2} \mathrm{~s}^{-1}$. At this level, around $10^{8} \mathrm{~B}$ mesons are 
produced each year and because the cross-section for $\tau^{+} \tau^{-}$production through $e^{+} e^{-} \rightarrow$ $\tau^{+} \tau^{-}(0.89 \mathrm{nb})$ is similar to the $\Upsilon(4 S) B \bar{B}$ production cross-section $(1.1 \mathrm{nb})$ there are approximately the same number of $\tau^{+} \tau^{-}$pairs produced too. The cross-section for continuum $q \bar{q}$ production at this energy is around $2.5 \mathrm{nb}$. In the data-taking periods between October 1999 and July 2002, just over $91 \mathrm{fb}^{-1}$ of physics quality data has been collected at or near the $\Upsilon(4 S)$ resonance. Before the scheduled shut down at the end of June 2003 this number had risen to $125.4 \mathrm{fb}^{-1}$.

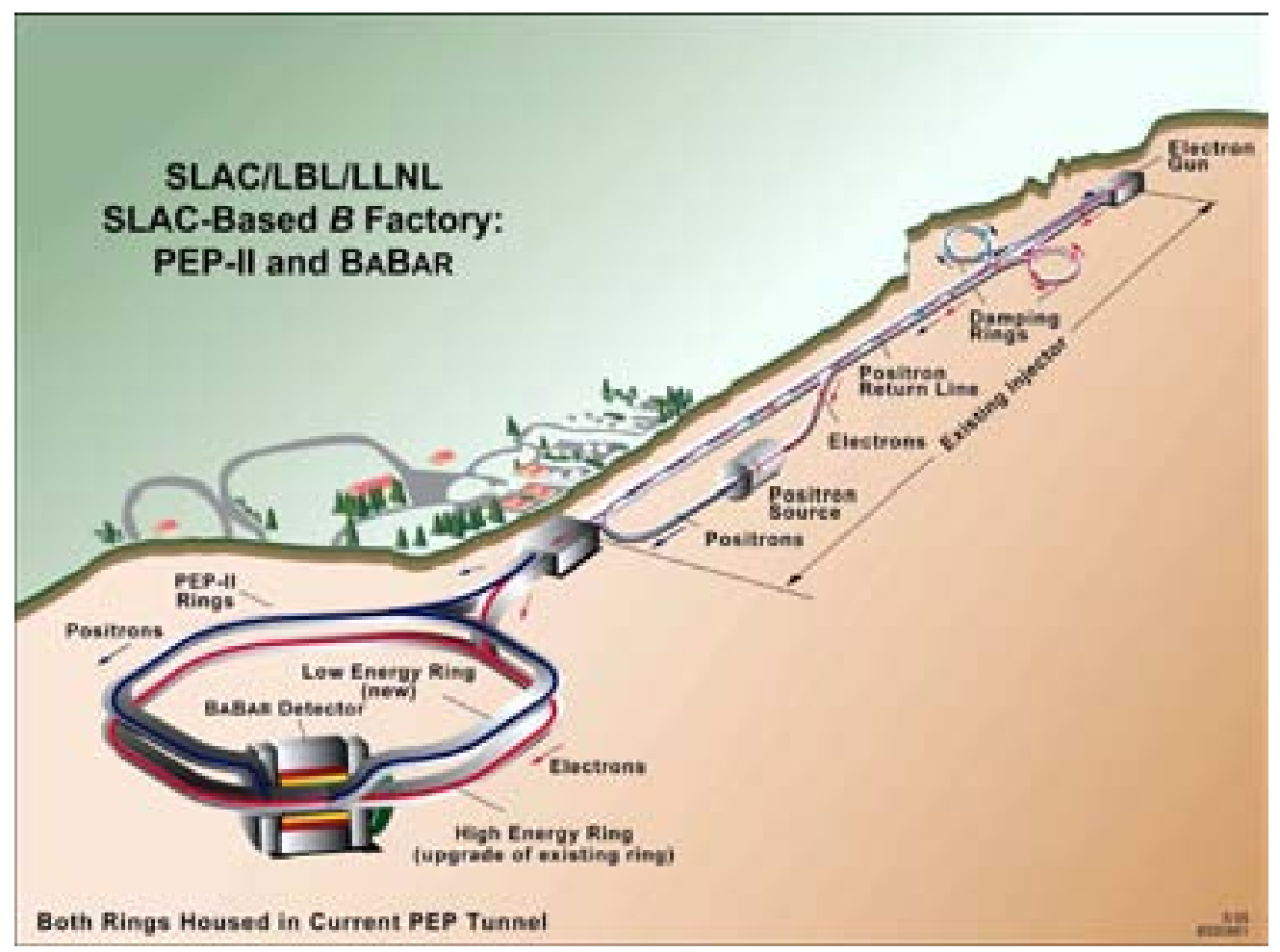

Figure 3.1: The PEP-II linear accelerator.

The $e^{+} e^{-}$collisions are asymmetric with a boost of $\beta \gamma=0.56$ along the collision axis. This means that the $B^{0}$ and $\bar{B}^{0}$ particles travel a short distance $(\sim 250 \mu \mathrm{m})$ before decaying, which makes their lifetimes easier to measure.

The SLAC linac is used as the main injector for PEP-II. It is necessary to "top off" the beam currents by injecting more current every 1-2 hours since there are losses in the rings. The time taken to increase the currents from $80 \%$ to $100 \%$ of their desired values is 
approximately two minutes. A complete fill is necessary if one or both of the beams are lost and takes approximately five minutes. Typical beam currents are $1 \mathrm{~A}$ in the HER and $1.7 \mathrm{~A}$ in the LER.

\subsubsection{The interaction region}

The design of the PEP-II interaction region is complex, due to the high luminosity and asymmetry of the PEP-II machine. Figure 3.2 shows a plan view of the interaction region.

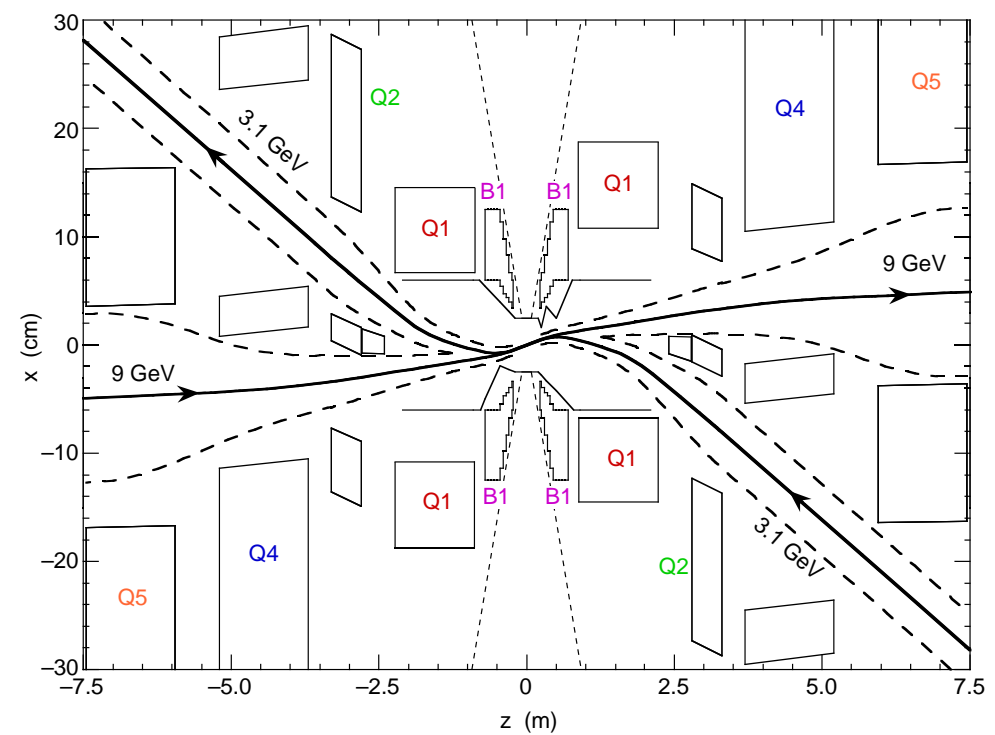

Figure 3.2: The PEP-II interaction region (exaggerated vertical scale). Q1-Q5 are focusing quadrupole magnets and B1 are separation dipoles.

It is essential that the beams collide only at the interaction point (IP). A sophisticated beam optics arrangement is implemented in order that the beams are successfully collided at the IP. Beam-beam interference is minimised by dividing the beams into a large number of low charge bunches; secondary collisions, which because of the bunch spacing would occur $62 \mathrm{~cm}$ from the IP, are avoided by bringing the beams together immediately before the IP and separated just after. In practice this is achieved using the separation dipole 
magnets (B1) which are placed close to the IP in order to displace the beams horizontally. This does, however, mean that these magnets are within the BABAR detector volume and so affect acceptance and backgrounds. Focusing of the beams is done by the quadrupole magnets: Q1 is the final focus for both beams; Q2 focuses the low energy beam, and; Q4 and Q5 focus the high energy beam. Only Q1 enters the detector volume, albeit only to a small extent.

\subsubsection{Machine backgrounds}

Large backgrounds are more prominent in the PEP-II accelerator than in many previous accelerators due to the large beam currents used to achieve the high luminosities. The beam backgrounds come from two main sources: synchrotron radiation due to constantly accelerating the charged particles by bending them in the magnetic field, and lost particle background produced by beam-gas interactions. Such large beam backgrounds provide an experimental challenge. Firstly, high occupancies in detector subsystems can occur which in turn leads to an increased amount of dead-time and therefore a loss in efficiency of detecting physics events. Secondly, subsystems must be radiation hard throughout the data-taking periods.

Synchrotron radiation effects are enhanced in the PEP-II design compared with most symmetric $e^{+} e^{-}$colliders due to the additional bending magnets near the IP. The geometry of the interaction region is designed so that the majority of the "fan radiation" passes through the detector without interacting. Copper masks are used to prevent such radiation interacting with the beam pipe.

The lost particle background is due to particles that fall outside the maximum momentum range of the storage rings hitting the beam pipe to produce electromagnetic showers. Interactions with beam-gas molecules are the main cause of this. This background can therefore be minimised by ensuring the gas pressure in the straight sections of the beam pipe near the interaction region is as low as possible. Collimators installed upstream from 
the detector in both directions help reduce backgrounds from particles hitting the beam pipe some distance from the IP.

\subsubsection{PEP-II performance}

During Run 1 (the data-taking periods between $22^{\text {nd }}$ October 1999 and $28^{\text {th }}$ October 2000) and Run 2 (the data-taking periods between $10^{\text {th }}$ February 2001 and $30^{\text {th }}$ June 2002) PEP-II performed exceptionally well. The design luminosity, both in terms of instantaneous luminosity and integrated daily luminosity, was exceeded in the first year of the experiment running. The peak luminosity delivered by PEP-II during this period was $4.602 \times 10^{33} \mathrm{~cm}^{-2} \mathrm{~s}^{-1}$. The record integrated luminosity over a 24 hour period was $308.8 \mathrm{pb}^{-1}$. During this time, PEP-II delivered an integrated luminosity of $98.58 \mathrm{fb}^{-1}$, of which BABAR recorded $93.80 \mathrm{fb}^{-1}$, out-performing a competing experiment, BELLE [82], that uses the KEKB accelerator [83] at KEK in Japan. The amount of data available for analysis after processing using 10 -series software is $91.6 \mathrm{fb}^{-1}$ of which $81.9 \mathrm{fb}^{-1}$ is onresonance and $9.6 \mathrm{fb}^{-1}$ is off-resonance, that is $40 \mathrm{MeV}$ below the $\Upsilon(4 S)$. This data, processed using 10-series software, was defined as the "summer 2002 data sample" and was used for a number of publications during most of 2002-3. The radiation doses received by all subsystems were also well within their budgets.

Unfortunately Run 3 (the data-taking periods between $8^{\text {th }}$ December 2002 and $30^{\text {th }}$ June 2003) encountered some reliability problems and the amount of data accumulated during this period is lower than expected, although daily luminosity records were broken. Nevertheless, the PEP-II delivered integrated luminosity for Run $1+$ Run 2 + Run 3 was $136.93 \mathrm{fb}^{-1}$, of which BABAR recorded $130.65 \mathrm{fb}^{-1}$ as shown in Figure 3.3. From Run 3 the extra amount of data available for analysis is $33.9 \mathrm{fb}^{-1}$ of which $31.4 \mathrm{fb}^{-1}$ is onresonance and $2.4 \mathrm{fb}^{-1}$ is off-resonance, therefore giving a Run $1+$ Run $2+$ Run 3 total of $125.4 \mathrm{fb}^{-1}$. This data has been processed (Run $1+$ Run 2 data reprocessed) using 
12-series software and this data set definition ${ }^{1}$ is now frozen for the next set of publications [84].

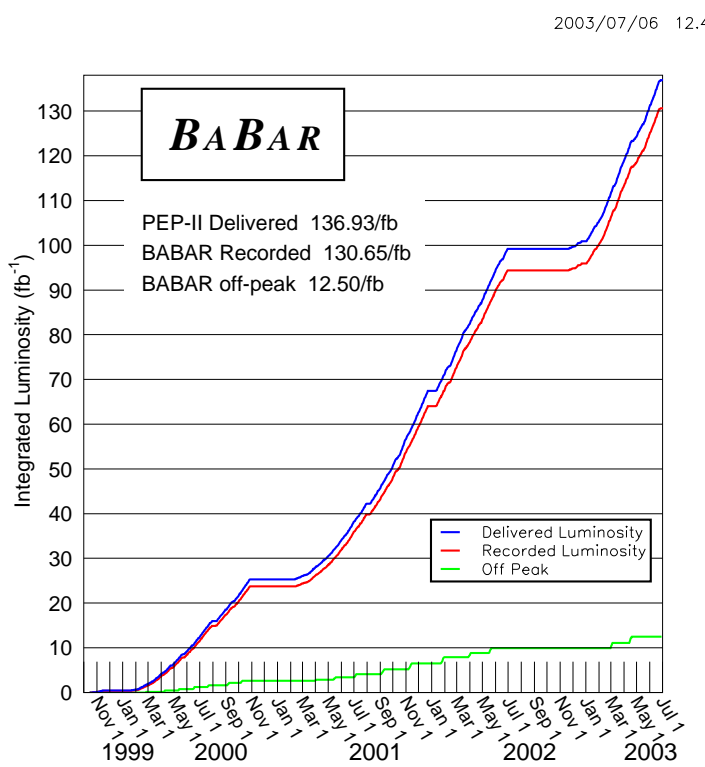

Figure 3.3: BABAR integrated luminosity during Run $1+$ Run $2+$ Run 3, the data-taking periods from October 1999 until June 2003.

\subsection{The BABAR detector}

\subsubsection{Overview}

The $B A B A R$ detector is highly complex and has to use many physics techniques to detect the various particles. A detailed description of the $B A B A R$ detector can be found in $[85,86]$.

To achieve the physics goals of the experiment, the detector must have excellent vertex resolution, a large centre of mass acceptance for particle detection and charged particle tracking over a large range of transverse momenta $(0.06-4 \mathrm{GeV} / \mathrm{c})$. It must also be able to identify charged particles $e, \mu, \pi, K, p$ and neutral particles, such as $\gamma$ and $\pi^{0}$, over the

\footnotetext{
${ }^{1} 12$-series data set defi nition: Run $1+$ Run 2: $91.607 \mathrm{fb}^{-1}\left(81.953 \mathrm{fb}^{-1}\right.$ on-resonance, $9.654 \mathrm{fb}^{-1}$ offresonance); Run 3: $33.829 \mathrm{fb}^{-1}$ (31.393 $\mathrm{fb}^{-1}$ on-resonance, $2.436 \mathrm{fb}^{-1}$ off-resonance).
} 
energy range $0.02-5 \mathrm{GeV}$.

Figure 3.4 and Figure 3.5 show the layout of the detector. In Figure 3.4 one can see that the higher energy electrons travel in the $+z$ direction and collide with the lower energy positrons that are travelling in the $-z$ direction. The collision occurs at the interaction point (IP). The detector has a number of subsystems, each designed to detect particular properties from which particle identification can be inferred. They are arranged in a layer ("onion") structure, Figure 3.5. Each subdetector is shown in Figure 3.4 and Figure 3.5. Their main physics roles are:

\section{$\diamond$ Silicon Vertex Tracker (SVT)}

The SVT provides a precise measurement of the position of charged particles with energies below $150 \mathrm{MeV}$. It consists of five cylindrical layers of silicon strip detectors and is located just outside the beam pipe. The spatial resolution of one of these silicon strip detectors is $80 \mu \mathrm{m}$.

\section{$\diamond$ Drift Chamber (DCH)}

The DCH is used for momentum measurement and track matching to the other subdetectors. Energy loss measurements $(\mathrm{d} E / \mathrm{d} x)$ help with particle identification for low energy tracks. The spatial resolution is $140 \mu \mathrm{m}$ and, for tracks with $p_{T}>$ $1 \mathrm{GeV} / c$, the momentum resolution is $0.3 \% \times p_{T}[\mathrm{GeV} / c]$.

\section{$\diamond$ Detector of Internally Reflected Čerenkov Light (DIRC)}

The DIRC is used to identify kaons for tagging purposes and also for $\pi / K$ separation at high energies. It uses quartz bars as Čerenkov radiators which transport the Čerenkov radiation out of the detector by total internal reflection. This radiation passes through the water expander at the back of the detector prior to detection by a photodiode array.

\section{$\diamond$ Electromagnetic Calorimeter (EMC)}

The EMC is designed to measure electromagnetic showers with excellent efficiency, energy and angular resolution over the energy range $0.02-9 \mathrm{GeV}$. It consists of a 
finely segmented array of thallium-doped caesium iodide (CsI(Tl)) crystals. The energy resolution, $\sigma_{E} / E$, is dependent on the energy: $\sigma_{E} / E \propto \frac{1}{\sqrt[4]{E}}$ approximately. So too the angular resolution has an energy dependence: $\sigma_{\theta=90^{\circ}}=\sigma_{\phi} \propto \frac{1}{\sqrt{E}}$. Since $\sigma_{\theta} \propto \frac{1}{\sin \theta}$, the $\theta$ resolution improves for tracks within the EMC volume that have $\theta \neq 90^{\circ}$.

\section{$\diamond$ Instrumented Flux Return (IFR)}

The iron flux return is the outer layer of the detector and is instrumented for the detection of $\mu$ and $K_{L}^{0}$ particles.

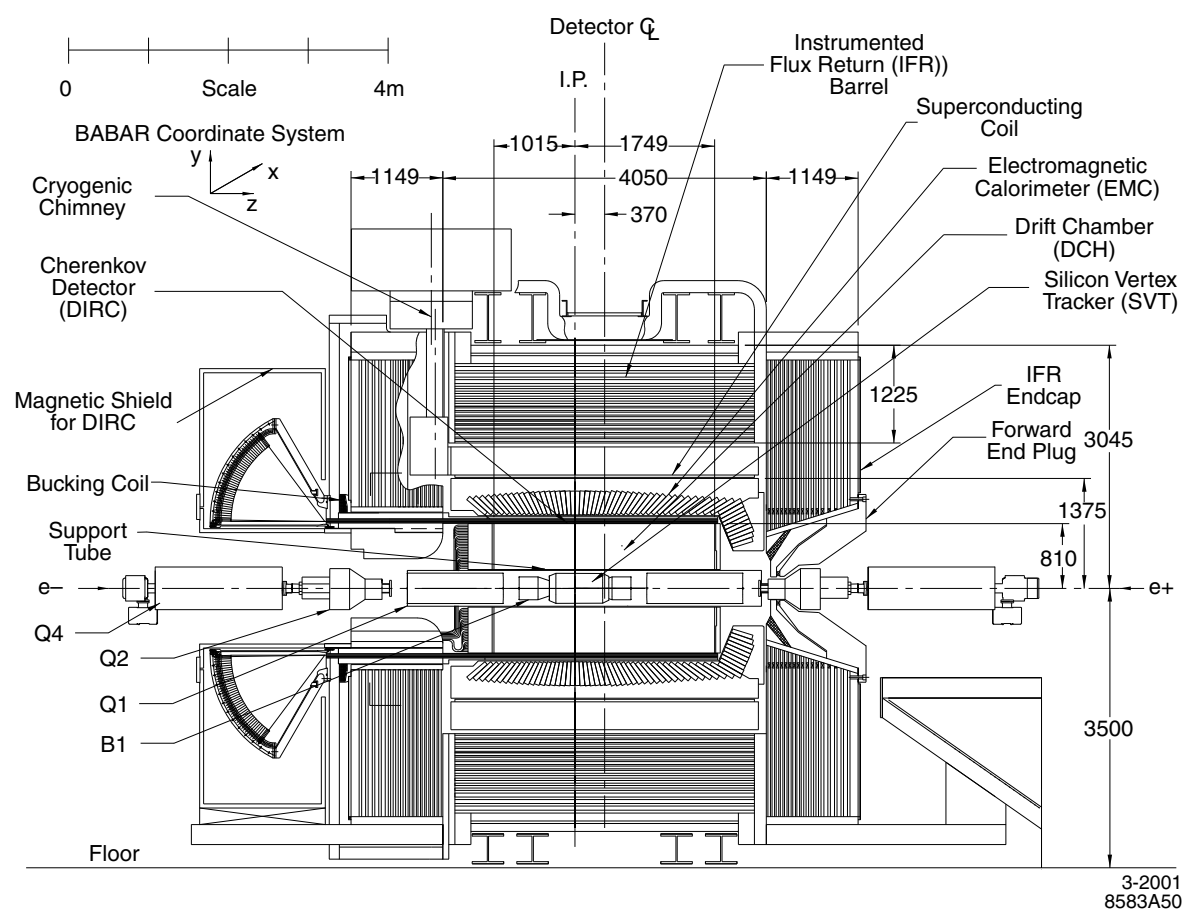

Figure 3.4: A side-view of the BABAR detector.

Due to momentum conservation, the resultant particles are generally forward-focused in the $+z$ direction so the priority for subsystems is to cover as much of this region as possible, but are limited by the beam pipe. This is why the EMC, for example, has endcaps on the forward end. 


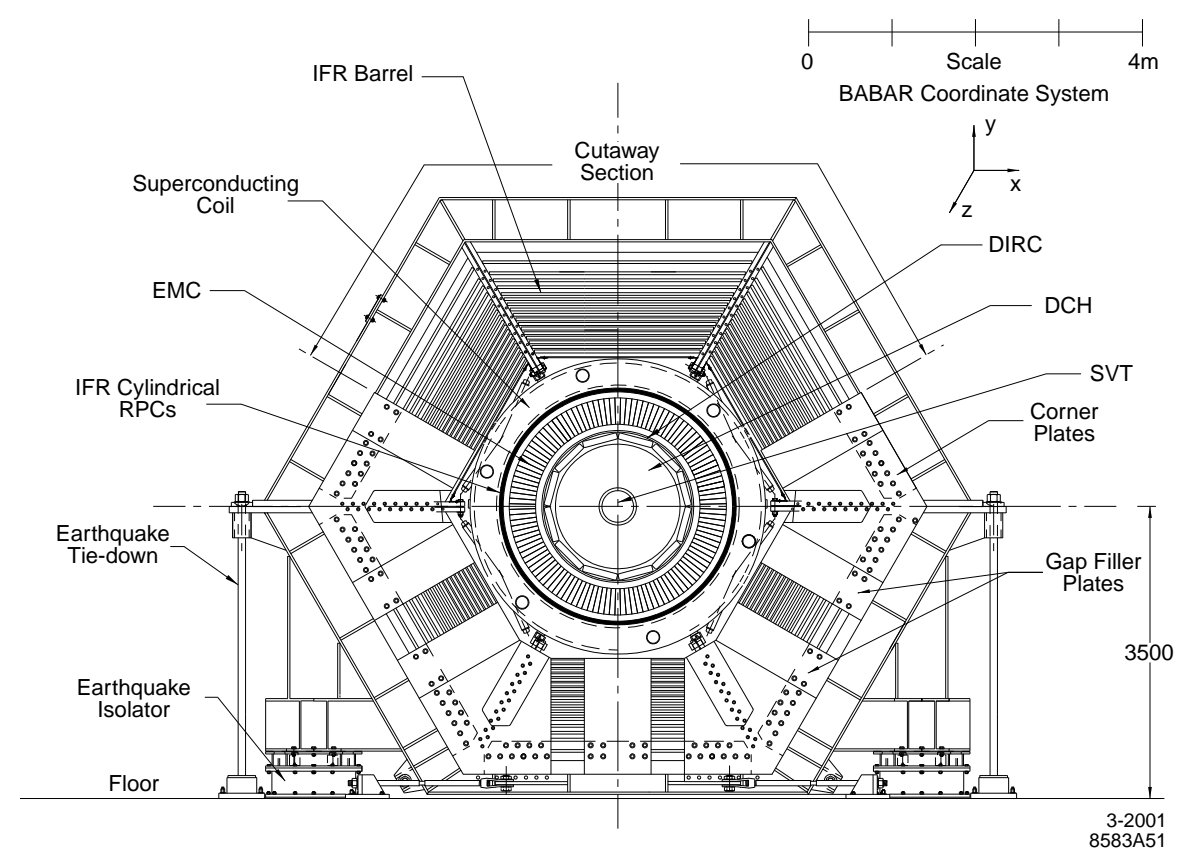

Figure 3.5: A cross-section view of the BABAR detector.

\subsubsection{The BABAR coordinate system}

$B A B A R$ uses a right-handed coordinate system with the origin at the IP and with the $z$-axis corresponding to the principal axis of the DCH. In both Figure 3.4 and Figure 3.5 the coordinate system is shown. The $y$-axis points vertically upwards and the $x$-axis points horizontally away from the centre of the PEP-II ring. The positive $z$ direction is the direction of the high energy (electron) beam with a slight shift in $x z$ of $20 \mathrm{mrad}$. This shift is a necessity of the PEP-II design in order to collide the beams from the two storage rings within the BABAR detector. $\theta$ and $\phi$ are the spherical polar coordinate relations to the Cartesian $x, y$ and $z$ coordinates. Therefore $\theta=0$ is along the $z$-axis whilst $\phi$ gives a measure of the direction perpendicular to the $z$-axis. 


\subsubsection{Silicon Vertex Tracker (SVT)}

\subsubsection{Physics requirements of the SVT}

Charged particle tracking at BABAR is made possible by the SVT and DCH subsystems which measure the momenta and angles of charged particles with high precision. The precision is sufficiently high that particle decay vertices can be reconstructed for particles with charged daughters. Track measurements are also important for the extrapolation to the DIRC, EMC and IFR. At lower momenta the SVT provides the principal measurements whereas for higher momenta the DCH measurements dominate. The success of the DIRC as particle identification apparatus depends critically on the uncertainties in the track parameters as measured by the SVT and DCH. These add to the uncertainty in the Čerenkov angle measurement and therefore uncertainties in the track parameters must be small compared to uncertainties in the Čerenkov angle, i.e. of order $1 \mathrm{mrad}$, especially at the highest momenta.

The Lorentz boost $(\beta \gamma=0.56)$ arising from the asymmetric beam energies means that the decay vertices of two $B$ mesons are expected to have a mean separation of $\sim 250 \mu \mathrm{m}$. The SVT measurements are therefore critical for measuring the time-dependent $C P$ asymmetry. To achieve sufficient resolution on the separation of the $B^{0}$ and $\bar{B}^{0}$ particles, Monte Carlo simulation studies have been conducted and show that the single vertex resolution along the $z$-axis should be better than $80 \mu \mathrm{m}$ [87,88]. Multiple scattering in the beryllium beam pipe limits the maximum achievable track parameter resolution. Good SVT resolution is also crucial to the $\tau$ lifetime measurement and for charm physics.

Tracks below $120 \mathrm{MeV} / c$ do not reach the DCH. The SVT is therefore solely responsible for their measurement and must be able to function as a standalone tracking device. Slow pions from $D^{*}$ meson decays are detected with a tracking efficiency of over $70 \%$ in the momentum range $50-120 \mathrm{MeV} / \mathrm{c}$ in the SVT only.

The SVT also aids particle identification. Not only does it provide the best measurement 
of track angle for high momentum tracks necessary for a good resolution of Čerenkov angle in the DIRC, but it also provides a measure of energy loss $(\mathrm{d} E / \mathrm{d} x)$.

\subsubsection{Design of the SVT}

The SVT consists of five concentric layers of double-sided silicon strip detectors divided azimuthally into 6, 6, 6, 16 and 18 modules respectively. On the opposite side of each sensor the strips are oriented orthogonally to one another: the $\phi$ measuring strips (" $\phi$ strips") run parallel to the beam and the $z$ measuring strips (" $z$ strips") are oriented transversely to the beam axis. Figure 3.6 shows a longitudinal and Figure 3.7 a transverse view of the SVT. The modules of layers 1, 2 and 3 are straight whereas the modules of layers 4 and 5 are arched. The arch structure increases solid angle coverage and reduces track incidence angles. However, the SVT acceptance is limited by the close proximity of the PEP-II beam optics as well as by mechanical supports, electronics and cabling. The resulting polar angle coverage is between $20.1^{\circ}-150.2^{\circ}$, with the lower limit governed by the $\mathrm{B} 1$ magnet positions.

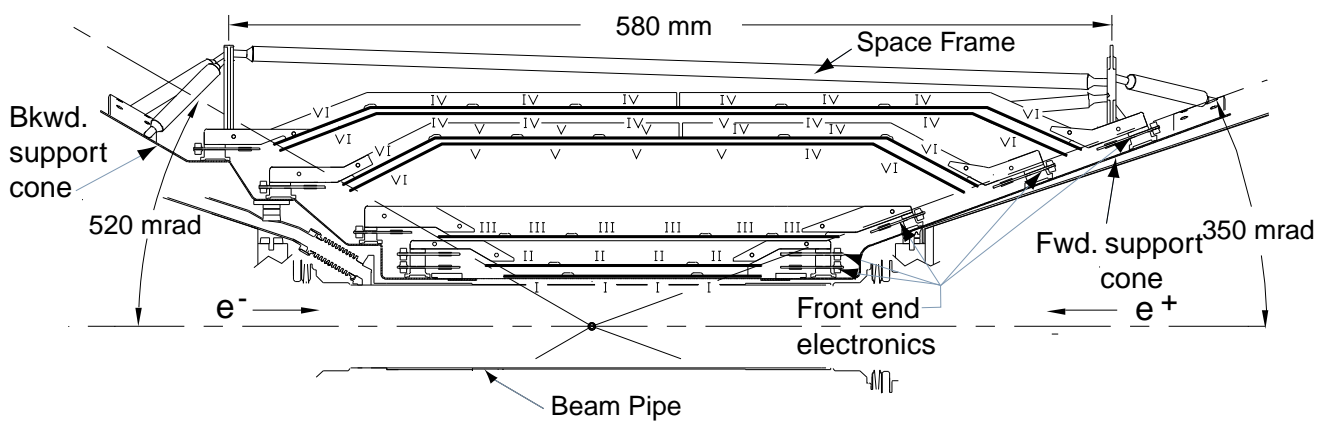

Figure 3.6: Longitudinal view showing the 5 layers of the SVT. The inner three layers are straight and the outer two layers are arched.

The modules are divided into electrically isolated forward and backward half-modules. The two inner layers' primary responsibility is to accurately measure the track angle. 


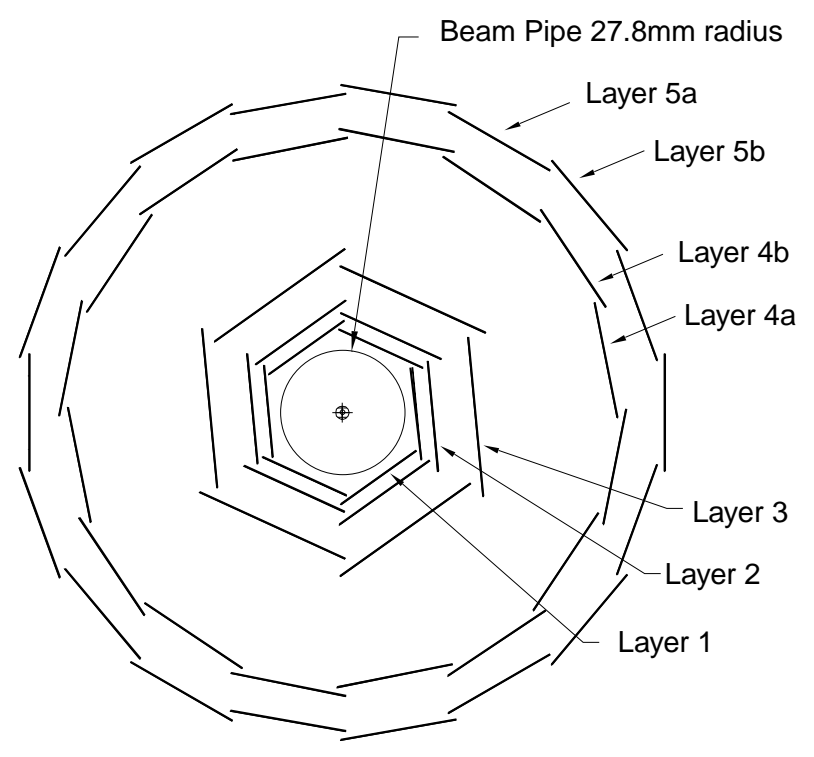

Figure 3.7: Transverse view showing the 5 layers of the SVT.

To maximise the accuracy, the measurement is made as close to the interaction point as possible so as to reduce the multiple scattering effects in the beam pipe. The two outer layers' main task is to align tracks with those found in the DCH. Additional tracking information is given by the third layer which is important for tracks that do not reach the DCH.

The SVT must be radiation hard throughout its working life. It must withstand an ionising radiation dose of $2 \mathrm{MRad}$. A radiation monitoring system that can absorb the beams is needed. The radiation dose is concentrated in the horizontal plane immediately outside the beam pipe. Here the average expected dose is $1 \mathrm{Rad} /$ day; elsewhere the average dose is expected to be an order of magnitude lower.

The electronic readout is performed by electronics attached to each half-module outside the detector acceptance. The front-end electronics use a time-over-threshold (TOT) method using the custom made ATOM ("A Time-Over-Threshold Machine") chip to determine the total charge deposited in a strip. A number of specifications had to be satisfied in designing the ATOM chip: a signal-to-noise ratio greater than 15 for minimum ionising 
particles; retain all signals from strips; an amplifier sensitive to both positive and negative charge; a programmable peaking time; an ability to accept random triggers with a programmable jitter and no dead time; radiation hardness greater than $2.5 \mathrm{MRad}$; small dimensions, i.e. to accommodate 128 channels in a chip of width $6 \mathrm{~mm}$. The signal from each strip is amplified and shaped. It is then compared to a threshold whose value depends on the background conditions. The TOT is logarithmically related to the charge deposited on the strips which enables a large dynamic range to be covered with only a small number of bits. The TOT is stored in a $11.5 \mu$ s trigger latency buffer (with a programmable jitter up to $\pm 1 \mu \mathrm{s})$. Upon receipt of a Level 1 accept command from the trigger system, the TOT and time is retrieved from the latency buffer by the data acquisition (DAQ) system.

Cooling is supplied to the SVT to remove heat generated by the electronics. The SVT must also be capable of operating in the $1.5 \mathrm{~T}$ magnetic field.

\subsubsection{Alignment of the SVT}

The alignment of the SVT is done in two steps: internally ("local alignment") which involves determining the relative positions of the 340 silicon sensors, and; with respect to the rest of the detector ("global alignment") as defined by the global coordinate system which is defined by the DCH. The local alignment constants are obtained using tracks from $e^{+} e^{-} \rightarrow \mu^{+} \mu^{-}$events, cosmic rays and high momentum hadronic events using only internal SVT information, i.e. using no DCH information, which effectively decouples the SVT and DCH alignment. They are changed relatively infrequently, typically after a magnet quenches or detector access, but can be changed when necessary. Data samples sufficient to perform local alignment are usually collected within one or two days of normal running.

After the local alignment is complete, the SVT is considered a rigid body. To obtain the global alignment constants, tracks with sufficient numbers of SVT and DCH hits are fitted twice: once using only the DCH information and again using only the SVT information. 
The six global alignment parameters, three translations and three rotations, are obtained by minimising the difference between track parameters obtained in these two fits. Since the DCH and SVT move a significant amount with respect to each other during datataking, this calibration is performed regularly ( $\sim 2-3$ hours) during the run. This rolling calibration procedure means that the resulting constants are used to reconstruct the data in the following run which ensures that track reconstruction is always performed with up-to-date global alignment constants.

\subsubsection{Performance of the SVT}

The SVT efficiency can be calculated for each half-module by comparing the number of associated hits to the number of tracks crossing the active area of the module. The combined hardware and software efficiency measured using data is $97 \%$. This figure excludes 9/208 defective readout sections but includes other defects such as broken AC coupling capacitors or dead channels on front-end chips. Since most of the tracks deposit charge in two or more strips these other defects tend not to cost efficiency since they only tend to affect one channel.

The spatial resolution of SVT hits is obtained by measuring the distance between the hit and the track trajectory in the plane of the sensor using high momentum tracks in twoprong events. The hit resolution is given by subtracting the uncertainty due to the track trajectory from the width of the residual distribution. The SVT hit resolution for $z$ and $\phi$ side hits as a function of track incidence angle for each of the five layers is shown in Figure $3.8 a$ and Figure $3.8 b$ respectively. Resolutions measured using the data agree well with Monte Carlo simulations. 

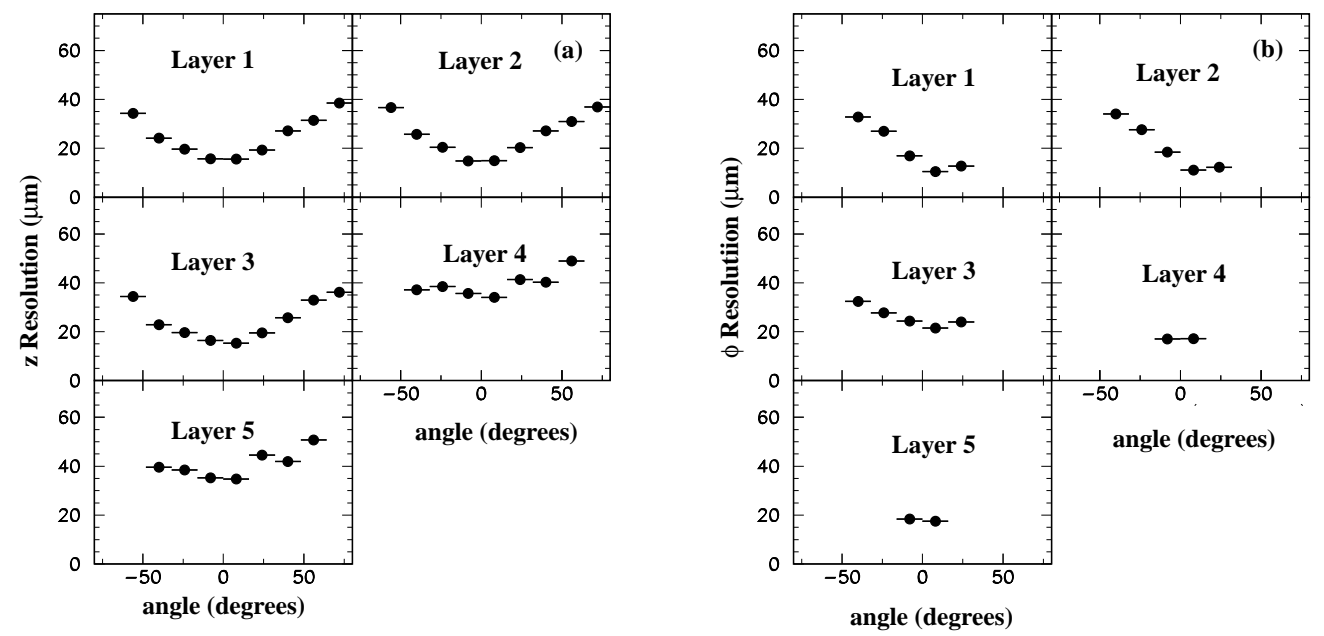

Figure 3.8: a) Track resolution in the $z$ coordinate (left) for each layer of the SVT as a function of track incidence angle. b) Track resolution in the $\phi$ coordinate (right) for each layer of the SVT as a function of track incidence angle. The plots for $\phi$ in layers 1-3 are asymmetric around $\phi=0$ due to the "pinwheel" design of the inner layers. As the outer layers subtend smaller angles than the inner layers there are fewer points in the outer layer plots.

\subsubsection{Drift Chamber (DCH)}

\subsubsection{Physics requirements of the DCH}

The main purpose of the DCH is the efficient detection of charged particles and the measurement of their momenta and angles with high precision. It is the primary tracking device of the BABAR detector. Moreover, the DCH complements the measurements of the impact parameter and the directions of the charged tracks provided by the SVT near the IP. The reconstruction of decay and interaction vertices outside the SVT volume, for example longer path length $K_{S}^{0}$ decays, relies solely on the DCH.

To achieve its physics goals, the DCH must be able to measure transverse momenta and positions effectively. It has a spatial hit resolution of $140 \mu \mathrm{m}$, and for tracks with $p_{T}>$ $1 \mathrm{GeV} / c$, the momentum resolution is $\sigma_{p_{T}} / p_{T}=(0.13 \pm 0.01) \% \cdot p_{T}[\mathrm{GeV} / c] \oplus(0.45 \pm$ 
$0.03) \%$. The longitudinal position of tracks is known with a resolution of $\sim 1 \mathrm{~mm}$.

The DCH must also provide $\mathrm{d} E / \mathrm{d} x$ information for use in particle identification. Such capability is particularly important at transverse momenta below $700 \mathrm{MeV} / c$ where the DIRC is ineffective. In addition, the solid angle coverage of the DCH should be as large as possible. It should also present the minimum amount of material possible in front of the other subsystems. Furthermore, the DCH provides one of the main inputs to the Level 1 trigger.

\subsubsection{Design of the DCH}

A longitudinal view of the DCH is shown in Figure 3.9. The design consists of a $276 \mathrm{~cm}$ long cylinder. By comparison the diameter of the $\mathrm{DCH}$ is relatively small: the inner radius is $23.6 \mathrm{~cm}$ and the outer radius is $80.9 \mathrm{~cm}$. There are 40 layers of small hexagonal cells providing up to 40 spatial and ionisation loss measurements for charged particles with $p_{T}>180 \mathrm{MeV} / c$. Coverage in the forward direction is increased by positioning the $\mathrm{DCH}$ asymmetrically about the IP.

The total thickness of material in the DCH is $1.08 \% X_{0}$ at normal incidence, where $X_{0}$ is the radiation length. A $1 \mathrm{~mm}$ thick beryllium inner surface presents $0.28 \% X_{0}$ to a track traversing it at $90^{\circ}$ to the beam axis. Material in the inner cylindrical wall is kept thin to aid the matching of the SVT and DCH tracks, to improve the track resolution and reduce the background from converted photons and interactions. The main structural element of the outer wall consists of two $1.6 \mathrm{~mm}\left(0.6 \% X_{0}\right)$ thick layers of segmented carbon fibre on a honeycomb core. The thin outer wall minimises the amount of material in front of the EMC and DIRC so as not to degrade their performance. At the ends there are flat endplates made from aluminium. The backward endplate thickness is $24 \mathrm{~mm}$. Directly behind the backward endplate are the preamplifier and digitiser electronics. The design of the forward endplate is slightly different in order to reduce the amount of material in front of the EMC endcap. Therefore, although an inner section of the forward endplate is 
$24 \mathrm{~mm}$ thick, the outer section (beyond a radius of $46.9 \mathrm{~mm}$ ) has a reduced thickness of $12 \mathrm{~mm}$.

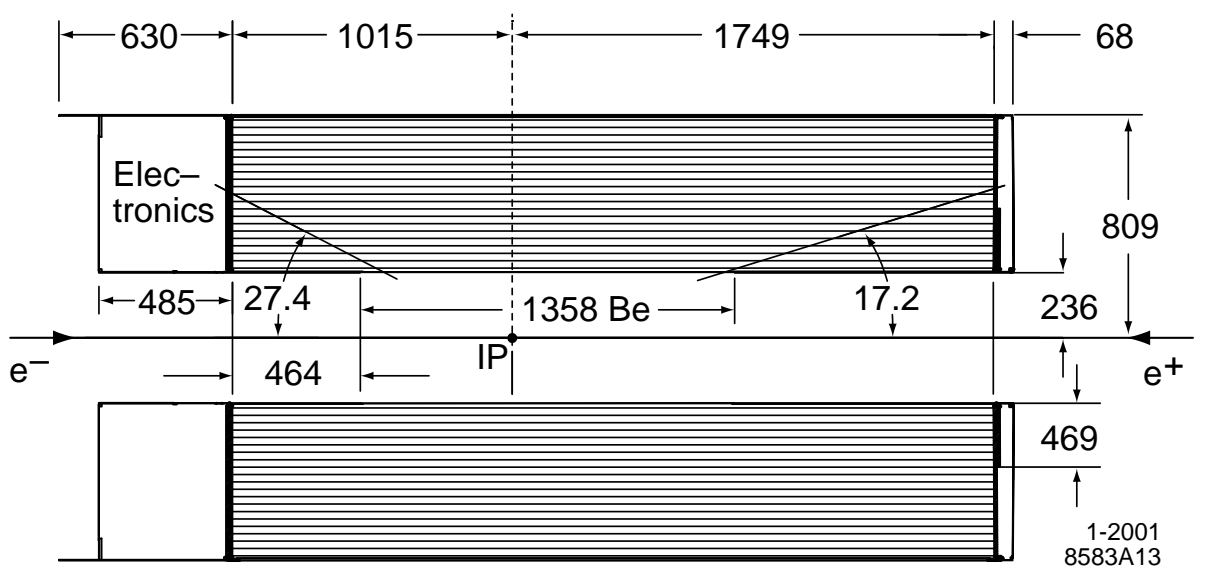

Figure 3.9: Longitudinal section of the BABAR DCH. All dimensions are in mm.

The gas mixture ratio helium:isobutane is $80: 20$. This particular mixture has a radiation length that is five times larger than commonly used argon-based gases. The gas mixture provides a smaller Lorentz angle which results in a uniform time-distance relationship. Moreover, a short drift time is achieved whilst minimising the amount of material in front of the other detector subsystems. The result is good spatial and $\mathrm{d} E / \mathrm{d} x$ resolution. In total, the wires and gas mixture contribute $0.2 \% X_{0}$ of material for a normal incidence track.

The DCH consists of a total of 7,104 small hexagonal drift cells arranged in 40 cylindrical layers. A drift cell is typically $11.9 \mathrm{~mm}$ in the radial direction by approximately $19.0 \mathrm{~mm}$ in the azimuthal direction. The layers are grouped by four into ten superlayers with each superlayer having the same wire orientation and equal numbers of cells. The stereo angles of the superlayers alternate between axial (A) and stereo $(\mathrm{U}, \mathrm{V})$ pairs in the order AUVAUVAUVA as shown in Figure 3.10. The stereo angles vary between $\pm 45 \mathrm{mrad}$ in the innermost superlayer to $\pm 76 \mathrm{mrad}$ at the outside.

The sense wires are $20 \mu \mathrm{m}$ in diameter, made of gold-plated tungsten-rhenium tensioned to a weight of $30 \mathrm{~g}$ and designed to operate at $1960 \mathrm{~V}$. At this voltage, and with the 
80:20 helium:isobutane gas mixture, a typical avalanche gain of approximately $5 \times 10^{4}$ is obtained. Tungsten-rhenium has a higher linear resistivity $(290 \Omega / \mathrm{m})$ compared with pure tungsten $(160 \Omega / \mathrm{m})$, but is used nonetheless as it has the advantages of being stronger and gives a better surface quality. Gravity causes a $200 \mu \mathrm{m}$ deflection at mid-length.

The field wires are $120 \mu \mathrm{m}$ and $80 \mu \mathrm{m}$ gold-plated aluminium and are at ground potential. They are tensioned with $155 \mathrm{~g}$ to match the gravitational sag of the sense wires to within $20 \mu \mathrm{m}$. The field-shaping wires at superlayer boundaries are at $340 \mathrm{~V}$.

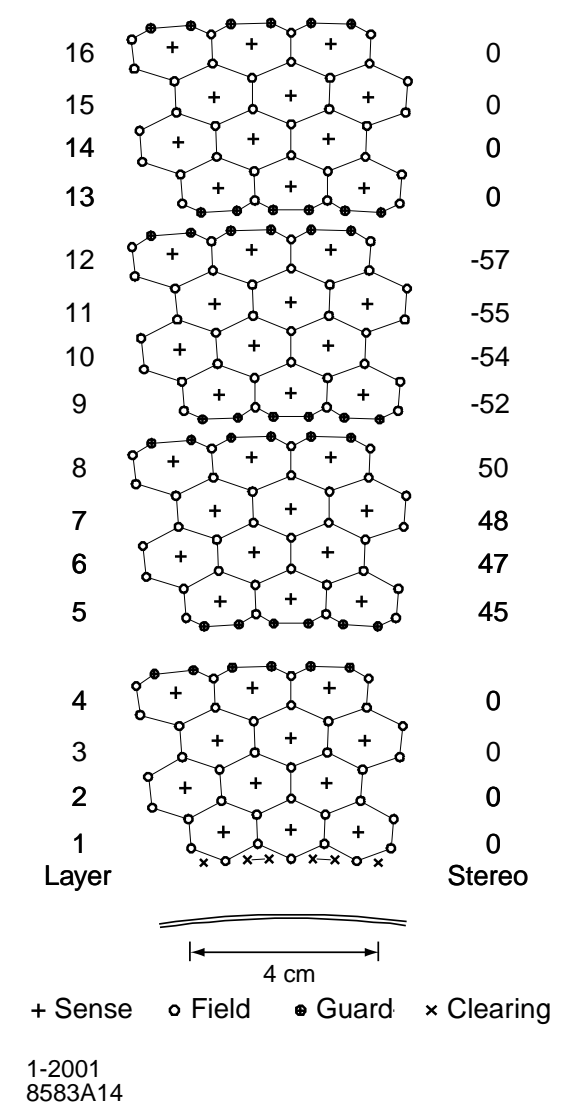

Figure 3.10: Cell layout of drift cells for the first four innermost superlayers $(A U V A)$ in the DCH. The layer number is given in the left hand column and the stereo angles of the sense wires in $\mathrm{mrad}$ are shown in the right hand column.

The spatial position of a track is calculated from the drift time measurement. To measure the drift time the electronics check for a leading edge of the signal from the sense wire. The signal is digitised with a $1 \mathrm{~ns}$ resolution. $\mathrm{d} E / \mathrm{d} x$ information for charged particles 
traversing the DCH is obtained by measuring the total charge deposited in each drift cell. The feature extraction algorithm measures the charge collected per signal cell. Individual measurements are corrected for gain variations, pedestal-subtracted and integrated over a period of approximately $1.8 \mu \mathrm{s}$. Numerous corrections are applied before data from all 7,104 channels are sent to the Level 1 trigger system. These corrections include changes in gas pressure and temperature, differences in cell geometry and charge collection, signal saturation due to space charge build-up, non-linearities in the most probable energy loss at large dip angles, and variation of cell charge collection as a function of entrance angle. The sampling frequency is $3.75 \mathrm{MHz}$.

\subsubsection{Performance of the DCH}

Samples of $e^{+} e^{-}$and $\mu^{+} \mu^{-}$events are used in order to relate the measured drift time to the drift distance. For each hit on a DCH wire, the drift distance is estimated by computing the point of closest approach between the wire and the track. To avoid bias, the fit does not use the hit on the wire under consideration. The estimated drift distances and measured drift times are averaged over all wires in a layer, although data are accumulated separately for tracks passing to the left and to the right of a sense wire.

Measurements of $\mathrm{d} E / \mathrm{d} x$ are also calibrated to remove biases in the total charge deposited in a cell arising from changes in gas pressure and temperature, from differences in cell geometry and from variations according to the entrance angle of the track into the cell. Figure 3.11 shows $\mathrm{d} E / \mathrm{d} x$ measurements as a function of momentum together with the expected Bethe-Bloch [89] curves for the five charged particle types superimposed. Particle identification is achieved using the $\mathrm{d} E / \mathrm{d} x$ measurements. Below $0.6 \mathrm{GeV} / c, \pi / K$ separation is good as shown in Figure 3.12. The root-mean-squared $\mathrm{d} E / \mathrm{d} x$ resolution for Bhabha events is typically $7.5 \%$, limited by the number of samples and Landau fluctuations. It is near to the expected value of $7 \%$.

In practice the voltage applied to the sense wires has been slightly different from the 


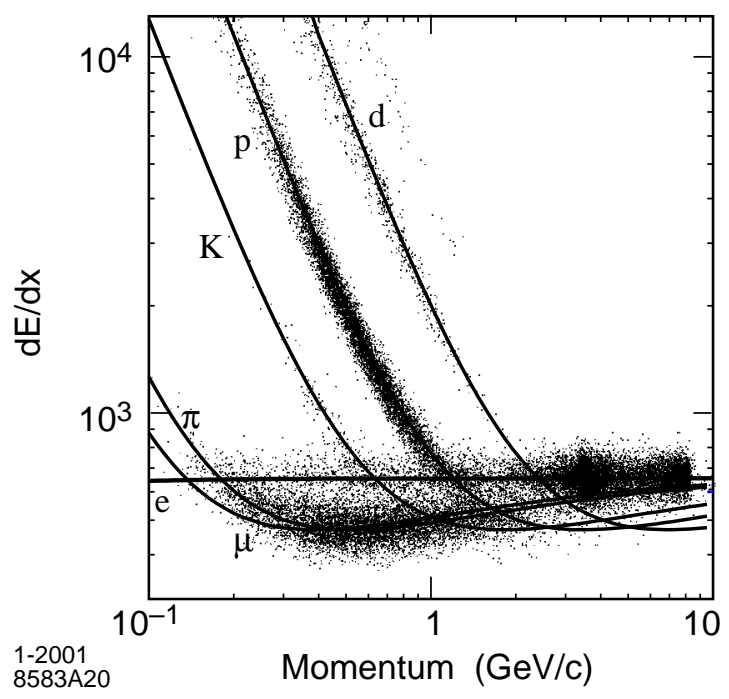

Figure 3.11: Measurement of $\mathrm{d} E / \mathrm{d} x$ in the DCH as a function of track momenta. The curves show the Bethe-Bloch predictions derived from selected control samples of each particle type.

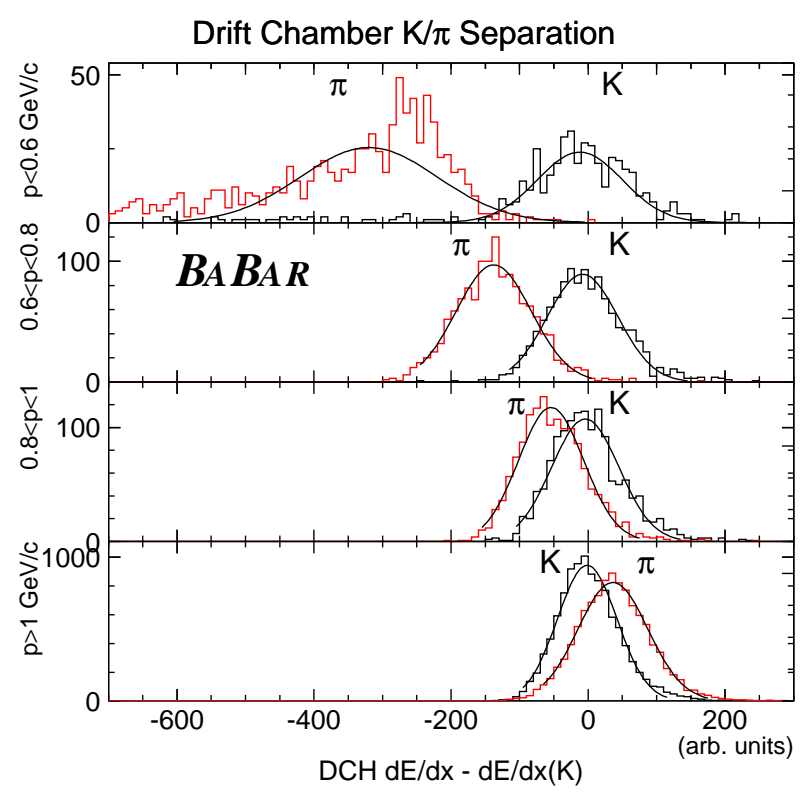

Figure 3.12: $D C H \mathrm{~d} E / \mathrm{d} x$ used for $\pi / K$ separation in track momenta, $p$, bins. For $p<0.6 \mathrm{GeV} / c$ the $\pi / K$ separation is particularly good.

design voltage of $1960 \mathrm{~V}$. For the first part of Run 1 a voltage of $1900 \mathrm{~V}$ was used after the chamber was slightly damaged during the commissioning phase. The wires in the 
damaged region (which covered $10 \%$ of superlayer 5 and $4.2 \%$ of superlayer 6 ) were disconnected so that the chamber could be operated at $1960 \mathrm{~V}$ in the latter part of 2000. Since January 2001 the sense wires have been operated at a voltage of $1930 \mathrm{~V}$.

\subsubsection{Charged particle tracking performance}

\subsubsection{Track reconstruction}

The reconstruction of charged particle tracks relies on SVT and DCH information. Charged tracks are defined by five parameters $\left(d_{0}, \phi_{0}, \omega, z_{0}, \tan \lambda\right)$ and their associated error matrix with respect to the DCH (global) coordinate system [90]. The parameters are defined in Table 3.1. $d_{0}$ and $\omega$ are signed quantities; their sign depends upon the charge of the track. The track finding and fitting procedures employ the Kalman filter algorithm [91]. In the procedure a detailed distribution of material in the detector and full map of the magnetic field is catered for.

\begin{tabular}{|c||c|}
\hline Parameter & Definition \\
\hline \hline$d_{0}$ & Distance from the POCA to the $z$-axis in the $x y$-plane. \\
$\phi_{0}$ & Azimuthal angle of the track at the POCA. \\
$\omega=1 / p_{T}$ & Curvature of the track at the POCA. \\
$z_{0}$ & Distance in the $z$-direction from the POCA to the origin. \\
$\tan \lambda$ & Tangent of the dip angle relative to the transverse plane. \\
\hline
\end{tabular}

Table 3.1: Definitions of the five track parameters, defined with respect to the DCH (global) coordinate system. POCA is used to abbreviate point of closest approach.

The offline track reconstruction uses information stored in the event from the Level 3 (L3) trigger and tracking algorithm. By fitting to $d_{0}, \phi_{0}$ and $t_{0}$ on the track segments in the DCH superlayers, an improved event start time value of $t_{0}$ is obtained. To select tracks, a helix parameterization is used in the fits to the hits found by the L3 track finding algorithm. Additional DCH hits associated with these tracks are searched for; $t_{0}$ is improved further 
by using only the tracks with associated hits. Two more sophisticated tracking procedures are applied whose algorithms primarily use track segments that have not already been assigned to other tracks. These benefit from a progressively cleaner tracking environment due to the iteratively improving value of $t_{0}$. The aim of these refinements is to find tracks that do not either pass through the whole DCH or originate from the IP. At the end of this process, the tracks are again fitted using a Kalman filter method.

The resulting tracks are then extrapolated into the SVT. SVT track segments that are consistent with the expected error in the extrapolation through the material and inhomogeneous magnetic field are added. Those track segments whose residuals are smallest and which contain hits in the largest number of SVT layers are kept and a Kalman fit is performed to the full set of DCH and SVT hits.

Two complementary standalone track finding algorithms make use of any remaining unassociated SVT hits. The first reconstructs tracks beginning with triplets of space points (matched $\phi$ and $z$ hits) in layers 1, 3 and 5 of the SVT, and adding consistent space points from the other layers. A good track requires a minimum of four space points. This algorithm is efficient over a wide range of $d_{0}$ and $z_{0}$. The second SVT track reconstruction algorithm starts with circle trajectories from $\phi$ hits and adds $z$ hits attempting to form helices. Sensitivity to large combinatorics and missing $z$ information for some of the SVT modules is reduced by this algorithm.

The final track reconstruction stage attempts to combine tracks found in either the DCH or SVT only. The aim is to recover any tracks scattered in the support tube material.

\subsubsection{Tracking efficiency}

Tracking efficiency measurements rely on specific final states and the ability to decouple the SVT and DCH track reconstruction. Measurements of the DCH efficiency have been made looking at the transverse momentum, polar and azimuthal angular dependence in 
multi-track events. The absolute DCH tracking efficiency is determined as the ratio of the number of reconstructed DCH tracks to the number of tracks detected in the SVT that fall within the acceptance of the DCH. At the design voltage of $1960 \mathrm{~V}$ it is found to be $(98 \pm 1) \%$ per track with $p_{T}>200 \mathrm{MeV} / c$ and $\theta>500 \mathrm{mrad}$.

The standalone SVT tracking algorithms have a high efficiency, even for low $p_{T}$ tracks. For the majority of these slow pions the resolution is limited by multiple scattering, but the production angle can be determined from the signals in the innermost layers of the SVT. Close agreement between the data and Monte Carlo $p_{T}$ spectra has enabled the detection efficiency to be derived from the Monte Carlo and shows that the SVT is a useful particle identification tool down to $p_{T} \sim 50 \mathrm{MeV} / c$.

\subsubsection{Track parameter resolutions}

The resolution of the five track parameters is monitored during online reprocessing (OPR) using Bhabha and dimuon events. Further investigation is done offline using tracks from multihadron events and cosmic ray muons. Cosmic rays that are recorded during normal data-taking provide a simple handle with which to measure the track parameter resolution. The upper and lower halves of the cosmic ray tracks are fit as two separate tracks and the track resolution is derived from the difference in the resulting track parameters. Table 3.2 shows the resolutions defined as the full width at half maximum of the distribution of differences between separate fits to the two halves of the tracks.

\begin{tabular}{|c||c|}
\hline Parameter & Resolution \\
\hline \hline$d_{0}$ & $23 \mu \mathrm{m}$ \\
$\phi_{0}$ & $0.43 \mathrm{mrad}$ \\
$z_{0}$ & $29 \mu \mathrm{m}$ \\
$\tan \lambda$ & $0.53 \times 10^{-3}$ \\
\hline
\end{tabular}

Table 3.2: Resolutions of charged track parameters measured using cosmic ray muons with $p_{T}>3 \mathrm{GeV} / c$. 
Using multihadron events, the resolution can be determined from the difference between the measured values of $d_{0}$ and $z_{0}$, and the coordinates of the vertex reconstructed from other tracks in the event. The resolution of $d_{0}$ and $z_{0}$ depends on $p_{T}$, as shown in Figure 3.13. To avoid bias from particle decays, only the negative side of the distributions are used.

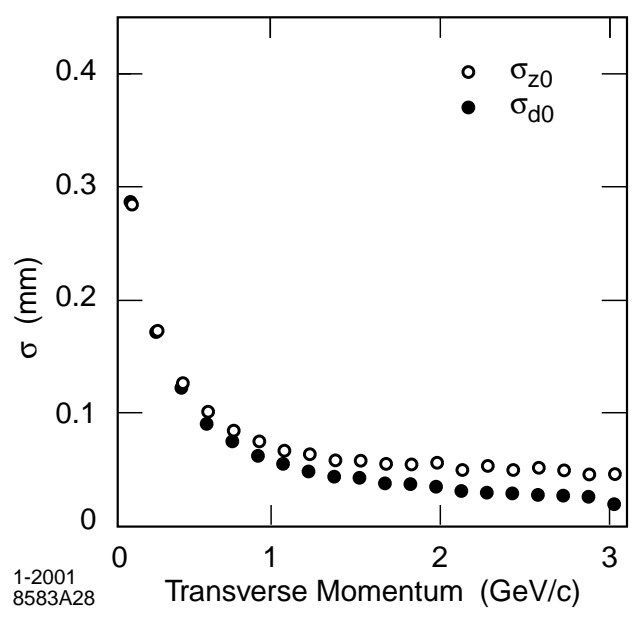

Figure 3.13: Resolution in the track parameters $d_{0}$ and $z_{0}$ as a function of transverse momentum. The data are from multihadron events and are corrected for the effects of particle decays.

\subsubsection{Detector of Internally Reflected Čerenkov Light (DIRC)}

\subsubsection{Physics requirements of the DIRC}

The DIRC has two primary roles: flavour tagging and particle identification. For $C P$ violation studies it is necessary to be able to tag the flavour of one $B$ meson via the cascade decay $b \rightarrow c \rightarrow s$, as well as fully reconstruct the other $B$ meson decay. The DIRC has an exceptional ability for $\pi / K$ separation. The momenta of the kaons used in the $B$ flavour tagging extend to $2 \mathrm{GeV} / c$, with most below $1 \mathrm{GeV} / c$, whilst the reconstruction of rare two-body decays $B^{0} \rightarrow \pi^{+} \pi^{-}$and $B^{0} \rightarrow K^{+} \pi^{-}$must be well-separated, which requires charged hadron identification for particles with momenta between $1.7-4.2 \mathrm{GeV} / \mathrm{c}$. More- 
over, the tracks' momenta and polar angles are highly correlated; higher momenta occur at more forward angles due to the Lorentz boost.

For tracks with $p_{T}>700 \mathrm{MeV} / c$ the $\mathrm{DCH} \mathrm{d} E / \mathrm{d} x$ measurements are no longer good enough to separate kaons and pions. The DIRC is the primary particle identification system for higher $p_{T}$ particles. At $p_{T}<750 \mathrm{MeV} / c$ the IFR is less efficient at identifying muons so the DIRC must also complement the IFR in this momentum region. The DIRC must therefore have a fast signal response in order to deal with high luminosities and must also tolerate high background levels.

Since the DIRC is located inside the EMC, it has to be both thin and uniform in terms of radiation length. This not only reduces degradation of the calorimeter energy resolution, but also reduces the size and therefore cost of the EMC.

\subsubsection{Design of the DIRC}

The DIRC uses the fact that the magnitudes of angles are preserved upon reflection from a flat surface. Consequently, Čerenkov photons trapped in the radiator by total internal reflection can be detected using photomultiplier tubes (PMTs). Even though this detection is done outside the detector volume the Čerenkov angle information remains intact. The DIRC principle is shown in Figure 3.14.

The DIRC radiator material is synthetic, fused silica (quartz) in the form of long, thin bars with rectangular cross-section. Fused silica was chosen as it is resistant to ionising radiation, has a long attenuation length, low chromatic dispersion within the wavelengths accepted by the DIRC, and admits an excellent optical finish on the surface of the bars. The bars are arranged in a barrel-like configuration with a water filled standoff box (SOB) at the back of the detector instrumented with PMTs.

The fused silica has a high refractive index $(n=1.473)$. A charged particle traversing a bar at velocity $\beta \geqslant 1 / n$ radiates a cone of Čerenkov photons at an angle of $\theta_{C}=1 / n \beta$, 


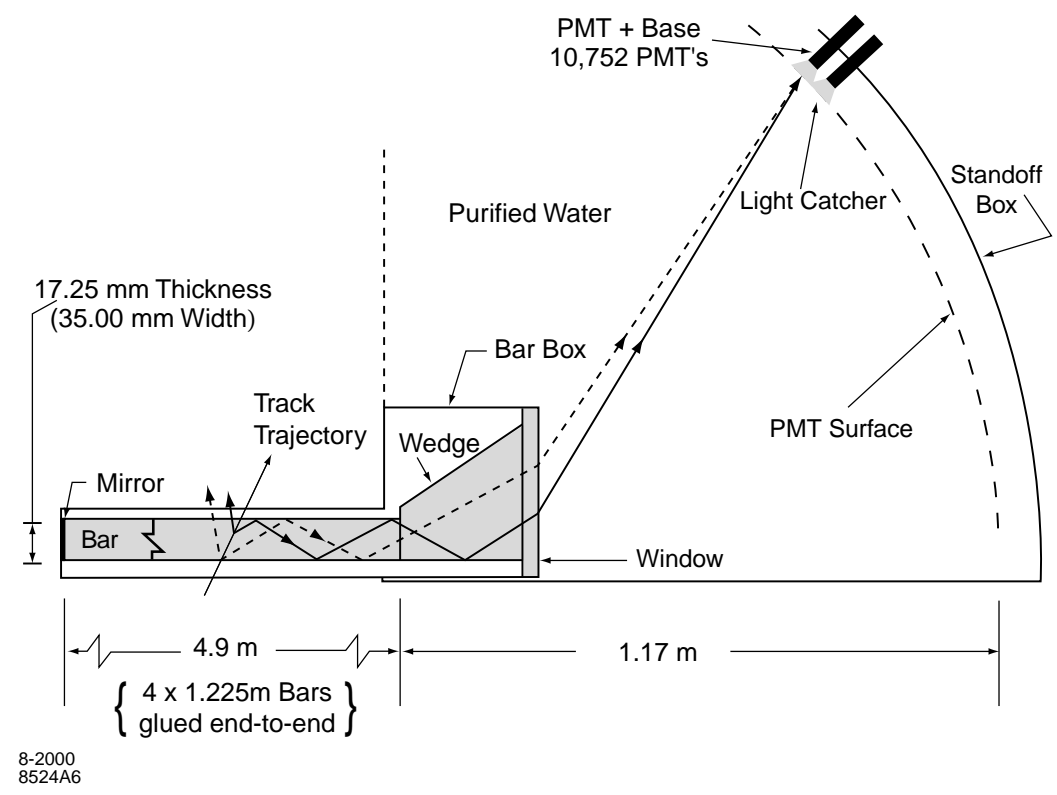

Figure 3.14: The DIRC fused silica and imaging section. Čerenkov photons produced by charged particles traversing the quartz bars travel along the bars via total internal reflection before arriving at an instrumented standoff box. The Čerenkov angle, $\theta_{C}$, is maintained during their journey.

where $\beta=v / c, v$ is the velocity of the particle in the medium and $c$ is the velocity of light in vacuum. For particles with $\beta \simeq 1$, some photons always lie within the total internal reflection limit and will successively be transported to either one or both ends of the bar, depending on the incidence angle of the particle. Forward going photons are reflected backwards by a mirror at the front end. When the Čerenkov light reaches the backward end of the bar, it expands for $1.17 \mathrm{~m}$ in the SOB and produces a Čerenkov ring image. The distance from the end of the bar to the PMTs is $\sim 1.17 \mathrm{~m}$, which together with the size of the bars and PMTs, gives a geometric contribution to the single photon Čerenkov angle resolution of $\sim 7 \mathrm{mrad}$. The highly purified water of the SOB has a refractive index ( $n \simeq 1.346$ ) and chromaticity index close to that of the quartz bars. This minimises the total internal reflection and dispersion at the quartz/water transition. Water also has the advantage of being cheap.

Each of the 144 quartz bars is $17 \mathrm{~mm}$ thick, $35 \mathrm{~mm}$ wide and $4.9 \mathrm{~m}$ long. The bars are 
arranged in a 12 sided barrel formation; each set of 12 bars is placed in a hermetically sealed "bar box" made of very thin aluminium. Within a bar box, the 12 bars are optically isolated from each other by a $\sim 150 \mu \mathrm{m}$ air gap.

Each PMT, of which there are 10,752 in 12 sectors lining the SOB, has a diameter of $29 \mathrm{~mm}$ and lies closely packed inside the water volume. The surface that they lie on is approximately toroidal. A trapezoidal wedge is glued to the readout end of each bar in order to reduce the required instrumentation area by folding one half of the Čerenkov ring image onto the other half. The wedge is made from the same material as the bar and the geometry is: $91 \mathrm{~mm}$ long, $33 \mathrm{~mm}$ wide and a trapezoidal profile $(27 \mathrm{~mm}$ at the high end of the bar and $79 \mathrm{~mm}$ at the light exit). A slight ( $\sim 6 \mathrm{mrad}$ ) upward displacement minimises the displacement of the downward reflected image due to the finite length of the bar. Hexagonal concentrators located in front of the PMTs increase the total effective surface area to around $90 \%$. Magnetic shielding is used to reduce the effect of the magnetic field on the PMTs.

Spreading the PMTs over such a large area ensures that at most only one photon per event hits them. As a result, only timing information is needed to characterise a PMT hit. Each of the 168 boards of the front-end electronics contains one time-to-digital converter (TDC) and two 8-channel custom chips which act as discriminators to time the PMT pulses. The boards are situated on the outside of the SOB. The TDCs digitise the time information and place the data into a buffer. This buffer is read out when a Level 1 accept command is received from the DAQ system.

\subsubsection{Performance of the DIRC}

The DIRC offers excellent $\pi / K$ separation power. From the measured single track resolution versus momentum in dimuon events and the difference between the expected Čerenkov angles of charged pions and kaons, it is possible to infer the $\pi / K$ separation power. The expected $\pi / K$ separation in $B^{0} \rightarrow \pi^{+} \pi^{-}$is shown in Figure 3.15. The ex- 
pected separation power between $3 \mathrm{GeV} / c$ kaons and pions is $\sim 4.2 \sigma$, which is within $15 \%$ of the design goal.

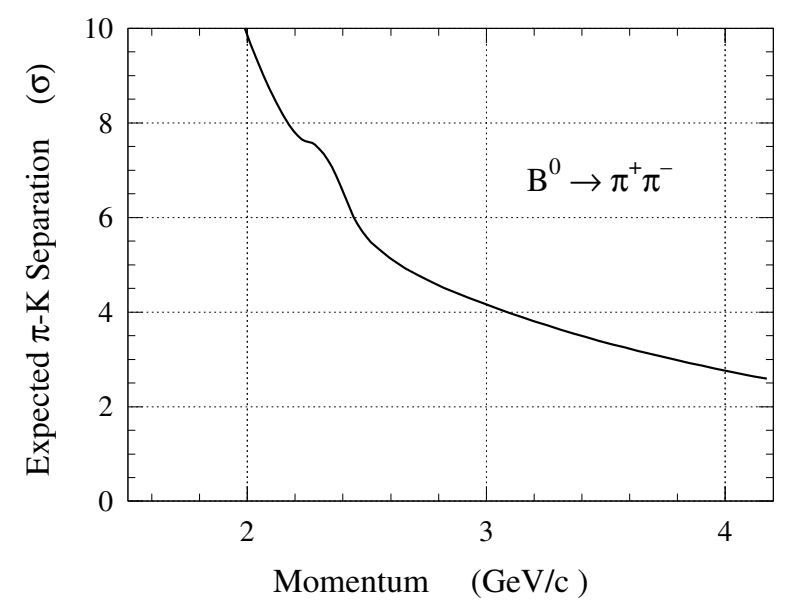

Figure 3.15: Expected $\pi / K$ separation in $B^{0} \rightarrow \pi^{+} \pi^{-}$events versus track momentum inferred from the Čerenkov angle resolution and the number of $\check{C}$ erenkov photons per track measured in dimuon events.

Figure 3.16 shows the advantage of invoking the DIRC as a tool for particle identification. In Figure 3.16 the $K \pi$ invariant mass spectra are shown both with and without the use of the DIRC for kaon identification for measuring the $K \pi$ mass in $D^{0}$ decays. The efficiency for correctly identifying a charged kaon and misidentifying a pion as a kaon have been determined using $D^{0} \rightarrow K^{-} \pi^{+}$decays selected kinematically from an inclusive $D^{*}$ sample. The result obtained was a mean kaon selection efficiency of $(96.2 \pm 0.2$ (stat) $) \%$ and corresponding pion misidentification rate is $(2.1 \pm 0.1$ (stat) $) \%$.

Overall, the DIRC has been performing reliably since its commissioning. About $99.7 \%$ of all PMTs and electronics channels are operating with nominal performance. The overall DIRC performance is close to that predicted by Monte Carlo simulations. 


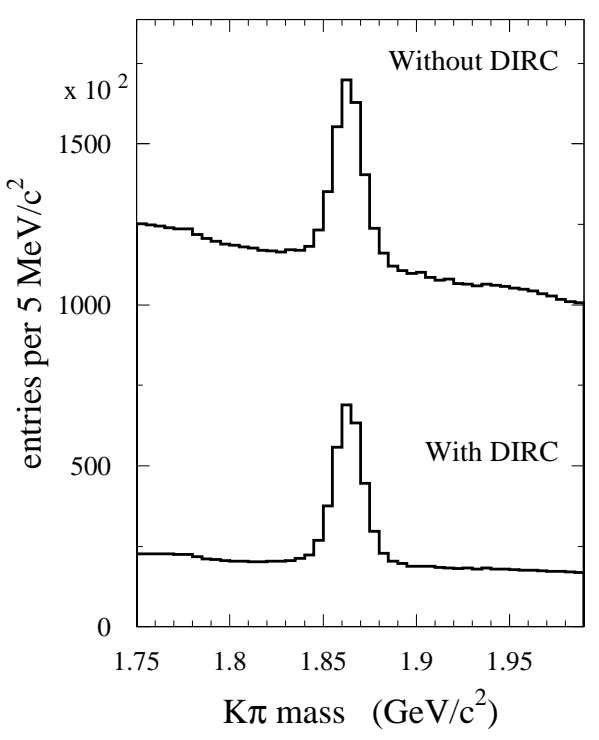

Figure 3.16: $K \pi$ invariant mass spectrum in the decay $D^{0} \rightarrow K^{-} \pi^{+}$without (top) and with (bottom) using DIRC information for kaon identification.

\subsubsection{Electromagnetic Calorimeter (EMC)}

\subsubsection{Physics requirements of the EMC}

The purpose of the EMC is to measure electromagnetic showers with excellent efficiency and both energy and angular resolution over the energy range $0.02-9 \mathrm{GeV}$. This capability allows the detection of photons from which neutral particles such as $\pi^{0} \mathrm{~s}$ and $\eta^{0} \mathrm{~s}$ can be reconstructed. The lower energy bound is set by this requirement. As well as the photons from decaying neutral particles, photons from electromagnetic and radiative QED processes can be detected. The QED processes $e^{+} e^{-} \rightarrow e^{+} e^{-}(\gamma)$ and $e^{+} e^{-} \rightarrow \gamma \gamma$ are needed for calibration and luminosity determination. They set the upper energy bound.

Even stricter energy resolution requirements $(\sim 1-2 \%)$ are imposed by the measurements of $B$ mesons containing more than one $\pi^{0}$, e.g. $B^{0} \rightarrow \pi^{0} \pi^{0}$, in order to achieve a high signal-to-background ratio. Below $2 \mathrm{GeV}$ the $\pi^{0}$ mass resolution is dominated by the energy resolution. At higher energies the angular resolution dominates and is required to 
be typically a few mrad.

The EMC also plays a vital role in electron identification. This is important for flavour tagging of neutral $B$ mesons via semileptonic decays, the reconstruction of vector mesons like the $J / \psi$ as well as semileptonic and rare decays of $B$ and $D$ mesons and also $\tau$ leptons.

\subsubsection{Design of the EMC}

The EMC comprises a barrel section and a conical forward endcap, necessary to cope with the forward-focused tendency of particle momenta due to the asymmetric beam energies. It is a hermetically sealed, total absorption calorimeter composed of a finely segmented array of 6,580 scintillating crystals made from caesium iodide doped with thallium (CsI(Tl)). $\mathrm{CsI}(\mathrm{Tl})$ was chosen as the scintillating material because of its high light yield and small Molière radius, confirmed by its proven success in the CLEO calorimeter. Some properties of the $\mathrm{CsI}(\mathrm{Tl})$ material are listed in Table 3.3. A high light yield helps achieve a good energy resolution and a small Molière radius helps the angular resolution. The crystals have a front face size of about $5 \mathrm{~cm}$ which is comparable to the Molière radius. The short radiation length allows showers to be contained in a relatively compact design. Moreover, the light yield and emission spectrum permit the efficient use of silicon photodiodes that are matched to the spectrum of the scintillation light. The silicon photodiodes are used for readout of the crystals. They work well in the $1.5 \mathrm{~T}$ magnetic field.

A cross-sectional view of the EMC is shown in Figure 3.17. The EMC has full coverage in azimuth and has a polar angle range of $15.8^{\circ} \leqslant \theta \leqslant 141.8^{\circ}$ corresponding to a solid angle coverage of $90 \%$ in the centre of mass system. This range equates to $-0.775 \leqslant$ $\cos \theta \leqslant 0.962$ in the LAB frame, corresponding to $-0.916 \leqslant \cos \theta^{*} \leqslant 0.895$ in the CMS frame. The EMC is positioned off-centre in $z$ and extends $2.3 \mathrm{~m}$ forwards and $1.56 \mathrm{~m}$ backwards from the IP. 


\begin{tabular}{|c||c|}
\hline Parameter & Values \\
\hline \hline Radiation length & $1.85 \mathrm{~cm}$ \\
Molière radius & $3.8 \mathrm{~cm}$ \\
Density & $4.53 \mathrm{~g} / \mathrm{cm}^{3}$ \\
Light yield & $50,000 \gamma / \mathrm{MeV}$ \\
Light yield temp. coeff. & $0.28 \% /{ }^{\circ} \mathrm{C}$ \\
Peak emission $\lambda_{\max }$ & $565 \mathrm{~nm}$ \\
Refractive index $\left(\lambda_{\max }\right)$ & 1.80 \\
Signal decay time & $680 \mathrm{~ns}(64 \%)$ \\
& $3.34 \mu \mathrm{s}(36 \%)$ \\
Radiation hardness & $10^{3}-10^{4} \mathrm{Rad}$ \\
\hline
\end{tabular}

Table 3.3: Properties of CsI(Tl) used to make the EMC scintillating crystals.

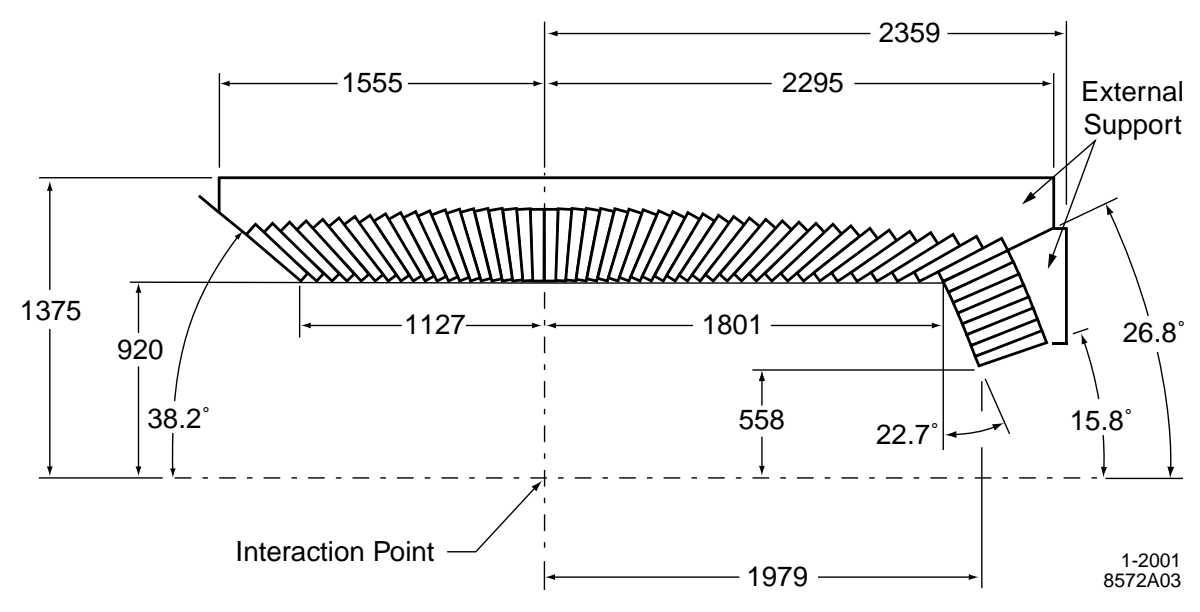

Figure 3.17: Longitudinal cross-section showing the EMC geometry (dimensions in $\mathrm{mm}$ ). The detector is axially symmetric about the z-axis.

Within the barrel section there are 5,760 crystals divided in $\theta$ into 48 distinct rings with 120 identical crystals in each. The inner radius is $91.9 \mathrm{~cm}$ and the outer radius is $135.6 \mathrm{~cm}$. Since the showers penetrate deeper in the forward direction, the crystal lengths vary with polar angle from $29.76 \mathrm{~cm}$ at the backward end to $32.55 \mathrm{~cm}$ at the forward end. The crystals are arranged into modules, each containing 21 crystals ( 7 in $\theta$ and 3 in $\phi$ ). In the endcap there are 820 crystals arranged in 8 rings, giving a total of 6,580 crystals. The crystals have a tapered trapezoidal cross-section with the length of the crystals increasing 
from $29.6 \mathrm{~cm}$ in the backward to $32.4 \mathrm{~cm}$ in the forward direction to limit the effects of shower leakage from increasingly higher energy particles. Preshowering is minimised by supporting the crystals at the outer radius with only a thin gas seal at the front. The barrel and outer five rings of the endcap have less than $0.3-0.6$ radiation lengths of material in front of the crystal faces. The three rings closest to the barrel each have 120 crystals in $\theta$, the next three have 100 crystals and the innermost two have 80 crystals each. All the crystals in the endcap are $32.55 \mathrm{~cm}\left(17.6 X_{0}\right)$ long, except for the inner ring which, due to space restrictions, is one radiation length shorter. There is also a ninth ring inside the endcap which contains lead shielding to reduce the effect of beam background on the endcap crystals.

Each crystal is read out by two silicon photodiodes glued to the back face. A preamplifier circuit receives the output and passes it on to an analogue-to-digital converter (ADC) board which digitises it into a 10-bit dynamic range. The data are sent via a high speed fibre optic Glink to the calorimeter readout modules (ROMs) in the BABAR electronics house. Data are collected continuously by untriggered personality cards (UPCs). A feature extraction algorithm is used to find the peak energy and time from a $1 \mu \mathrm{s}$ data sample before and after a Level 1 accept signal has been received. The data on the UPCs are also used to provide trigger tower sums for the Level 1 calorimeter trigger (EMT).

The energy resolution of a homogeneous crystal calorimeter can be parameterized empirically as the sum of two terms added in quadrature:

$$
\frac{\sigma_{E}}{E}=\frac{a}{\sqrt[4]{E[\mathrm{GeV}]}} \oplus b,
$$

where $E$ and $\sigma_{E}$ refer respectively to the photon energy and its RMS error measured in $\mathrm{GeV}$. The energy dependent term, $a$, is dominant at low energies. It arises primarily due to fluctuations in photon statistics but is also affected by electronic noise from the photon detector and other electronics. Moreover, backgrounds generated by the beam lead to large numbers of additional photons that add to the noise. The constant term, $b$, is dominant at energies above $1 \mathrm{GeV}$. It arises due to the non-uniformity in light collection, leakage or absorption in the material between and in front of the crystals, and uncertainties 
in the calibrations. At low energy, the energy resolution of the EMC is measured directly with a radioactive source; at high energy, Bhabha scattering is used.

The angular resolution is determined by the transverse crystal size and the distance from the interaction point. Its measurement is based on the analysis of $\pi^{0}$ and $\eta^{0}$ decays to two photons of approximately equal energy. The resolution varies between about $12 \mathrm{mrad}$ at low energies to $3 \mathrm{mrad}$ at high energies. The angular resolution can be parameterized as the sum of a constant term and an energy dependent term:

$$
\sigma_{\theta=90^{\circ}}=\sigma_{\phi}=\frac{c}{\sqrt{E[\mathrm{GeV}]}} \oplus d .
$$

For tracks in the EMC volume away from $90^{\circ}$, the $\theta$ resolution improves:

$$
\sigma_{\theta}=\frac{\sigma_{\theta=90^{\circ}}}{\sin \theta} .
$$

\subsubsection{EMC reconstruction algorithms}

An electromagnetic shower coming from an electron or photon will typically spread over many crystals, forming a cluster of energy deposits. A cluster is defined as the energy deposit caused by one or more particles interacting in the EMC. It is a contiguous set of crystals with recorded energy. At least one seed crystal with an energy above $10 \mathrm{MeV}$ is needed to make a cluster. Surrounding crystals are included in the cluster if their energy exceeds $1 \mathrm{MeV}$ or if they are contiguous neighbours (including corners) of a crystal that has an energy over $3 \mathrm{MeV}$. These thresholds arise from a compromise which balances the requirements of good energy resolution with the data volume limitations of the DAQ system. The EMC contributes to particle identification (PID) using the total energy of clusters and their lateral distribution.

Clusters can be divided into bumps. A bump is defined as a local maximum within a cluster and is intended to correspond to the fraction of the cluster energy deposited by a single particle interaction. Clusters, therefore, frequently contain more than one bump, i.e. if there is more than one local maximum within it. In this case, pattern recognition 
algorithms are employed to assign the correct energy to each bump. The algorithm also determines whether the bump comes from a charged or neutral particle.

Local energy maxima within a cluster are realised if the energy $E_{\text {LocalMax }}$ exceeds that of their neighbours and satisfies the condition $0.5(N-2.5)>E_{\mathrm{NMax}} / E_{\text {LocalMax }}$, where $E_{\mathrm{NMax}}$ is the highest energy of any of the $N$ neighbouring crystals with an energy above $2 \mathrm{MeV}$. An iterative procedure is used to determine the energy of each bump, with each crystal being assigned a weight. The bump energy is then the sum of the weighted crystal energies, running over all the crystals in the cluster. Their positions are calculated using a centre-of-gravity method with logarithmic weights. This method leads to a systematic bias in the polar angle due to the non-projectivity of the crystals. An offset correction of $-2.6 \mathrm{mrad}$ for $\theta>90^{\circ}$ and $+2.6 \mathrm{mrad}$ for $\theta<90^{\circ}$ is therefore applied.

A bump is associated with a charged particle by projecting a track to the inner face of the EMC. The distance between the track impact point and the centroid of a bump is calculated. If it is consistent with the angle and momentum of the track, the bump is associated with the charged particle. Bumps that are not associated to any tracks are assumed to be from neutral particles. On average, 15.8 clusters are detected per hadronic event, of which 10.2 are unassociated.

\subsubsection{Performance of the EMC}

The EMC output is continuously monitored and calibrated. A fibre optic light-pulser system is used daily to monitor short term changes in the detector response at the $0.15 \%$ level. There is also a source calibration performed weekly. A neutron generator provides a low energy flux of neutrons which irradiates a liquid source of Fluorinert being pumped around the crystals, producing the isotope ${ }_{7}^{16} \mathrm{~N}$ and an $\alpha$ particle. The $\beta$-decay process ${ }_{7}^{16} \mathrm{~N} \rightarrow{ }_{8}^{16} \mathrm{O}^{*}+{ }_{-1}^{0} \beta$ ensues, the half-life of which is 7 seconds. There is then a radiative

transition, ${ }_{8}^{16} \mathrm{O}^{*} \rightarrow{ }_{8}^{16} \mathrm{O}+\gamma$, to the ground state with the emission of a $6.13 \mathrm{MeV}$ photon. As this energy is precisely known, the EMC energy resolution at low energies 
can be determined to within $0.25 \%$.

Calibration is also done using physics processes themselves. Bhabha events provide an effective calibration method at high energy $(\sim 7.5 \mathrm{GeV})$ since the actual energies of the electrons can be calculated from their polar angles. Radiative Bhabha events and $\pi^{0}$ decays are also used as calibration tools over a wide energy range.

In Figure $3.18 a$ the EMC energy resolution as a function of energy is shown. The parameters of Eq. (3.1) extracted from this fit to the energy dependence yield $a=(2.32 \pm 0.30) \%$ and $b=(1.85 \pm 0.12) \%$. These values agree with the detailed Monte Carlo simulations which include electronic noise and beam background but are not quite as good as design expectations ( $a=1 \%, b=1.2 \%)$.

The angular resolution parameters in Eq. (3.2) have been extracted from the fit to an empirical parameterization of the energy dependence, Figure 3.18b. The values of the parameters are $c=(3.87 \pm 0.07) \mathrm{mrad}$ and $d=(0.00 \pm 0.04) \mathrm{mrad}$, which compare favourably with the design values ( $c=3 \mathrm{mrad}, d=2 \mathrm{mrad}$ ). The fitted values are slightly better than expected from Monte Carlo simulations.

For $B \bar{B}$ events, a reconstructed measured $\pi^{0}$ mass of $135.1 \mathrm{MeV} / c^{2}$ is stable to better than $1 \%$ over the whole photon energy range. The $\pi^{0}$ resolution of $6.9 \mathrm{MeV} / c^{2}$ agrees well with the Monte Carlo prediction obtained from the detailed detector simulation. These values are extracted from a fit to the data as shown in Figure 3.19. A slight improvement in the $\pi^{0}$ resolution (6.5 MeV/c $c^{2}$ ) is observed in low occupancy $\tau^{+} \tau^{-}$events for $\pi^{0}$ energies below $1 \mathrm{GeV}$. 

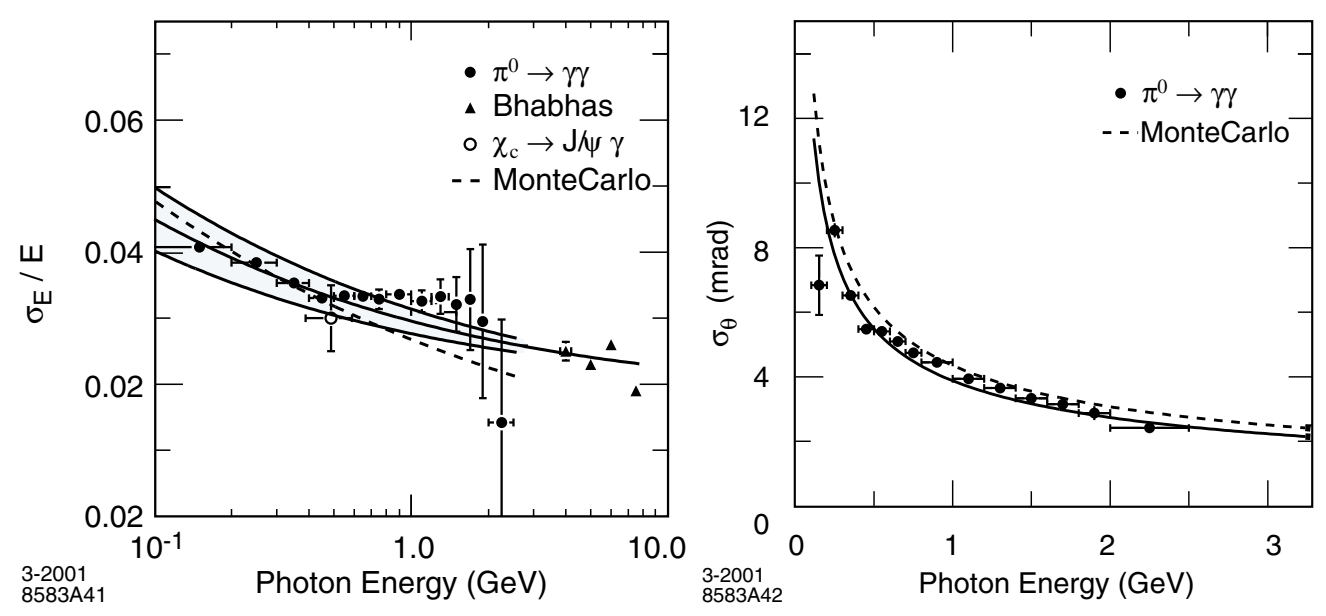

Figure 3.18: a) Energy resolution (left) of the EMC measured for photons and electrons from various physics processes. The solid curve is a fit to Eq. (3.1) and the shaded area represents the RMS error of the fit. b) Angular resolution (right) of the EMC measured for photons from $\pi^{0}$ decays. The solid curve is a fit to Eq. (3.2).

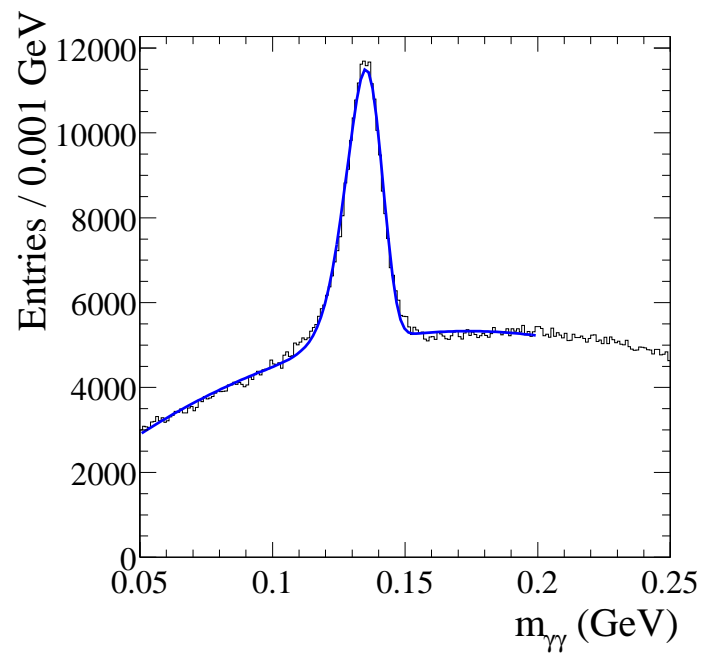

Figure 3.19: Invariant mass in $\mathrm{GeV} / c^{2}$ of two photons in $B \bar{B}$ events. The energies of the photons are required to be above $30 \mathrm{MeV}$ and the $\pi^{0}$ energy above $300 \mathrm{MeV}$. A fit to the data is shown by the solid line. 


\subsubsection{Instrumented Flux Return (IFR)}

\subsubsection{Physics requirements of the IFR}

The purpose of the IFR is to identify muons with high efficiency and purity, and to detect neutral hadrons (mainly $K_{L}^{0}$ particles and neutrons) over a wide range of momenta and angles. Reliable muon identification is useful for $B$ flavour tagging via semileptonic decays, reconstructing vector mesons like the $J / \psi$, and to study semileptonic and rare decays of $B$ and $D$ mesons and also $\tau$ leptons. IFR information is therefore important to many $B A B A R$ analyses. For example, $K_{L}^{0}$ detection allows exclusive $B$ decays, which include $C P$ eigenstates, to be studied.

The IFR is required to have large solid angle coverage, good efficiency and background rejection for muons with momenta down to below $1 \mathrm{GeV} / c$. For neutral hadron detection, high efficiency and angular resolution are the most important specifications.

\subsubsection{Design of the IFR}

The IFR is the outermost active part of the BABAR detector. It uses the steel flux return of the $1.5 \mathrm{~T}$ magnetic field as a muon filter and hadron absorber. Between the gaps of the finely segmented steel of the hexagonal barrel and end doors of the flux return are resistive plate chambers (RPCs), Figure 3.20. The steel is segmented into 18 plates of increasing thickness; $2 \mathrm{~cm}$ for the inner nine and $10 \mathrm{~cm}$ for the outermost plates. This segmentation was determined by Monte Carlo simulation studies to optimise both muon and neutral hadron detection. There is a $3.5 \mathrm{~cm}$ nominal gap between the plates in the inner layers of the barrel and $3.2 \mathrm{~cm}$ gap elsewhere. The solid angle coverage goes down to $300 \mathrm{mrad}$ in the forward direction and $400 \mathrm{mrad}$ in the backward direction.

There are 19 RPC layers in the barrel and 18 layers in the endcap. In addition, there are two layers of cylindrical RPCs installed between the EMC and the magnet cryostat to 


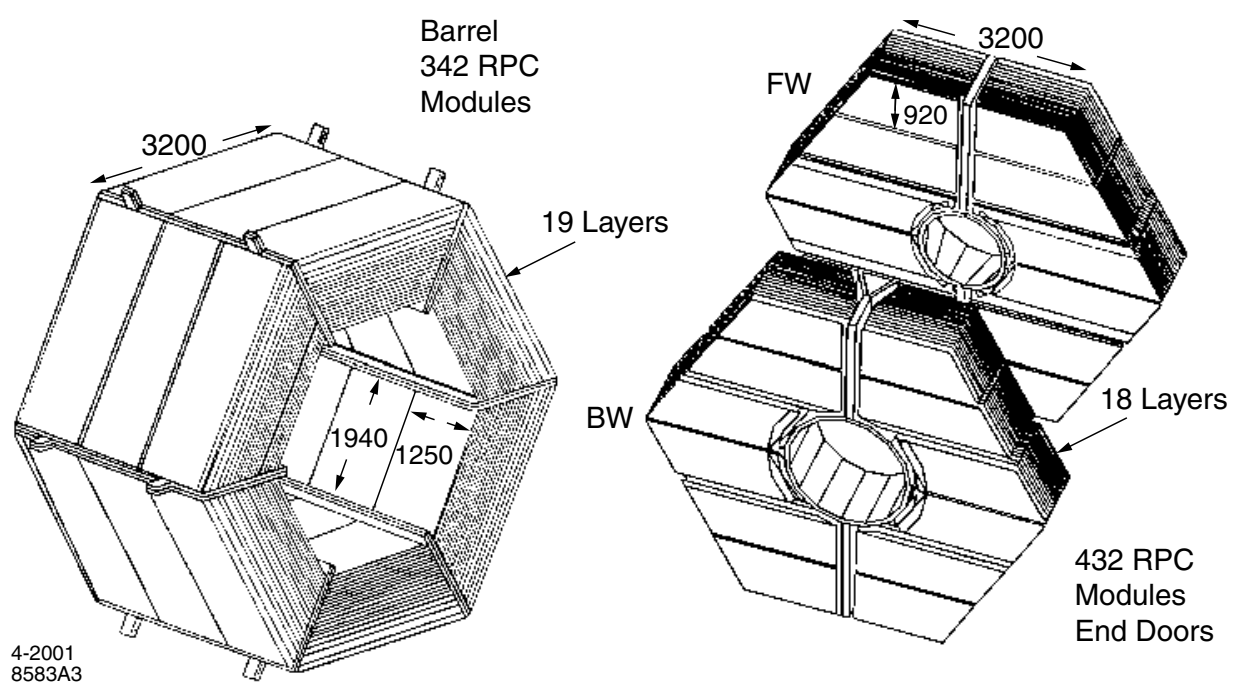

Figure 3.20: Geometry of the IFR showing the barrel and end doors (dimensions in $\mathrm{mm}$ ). The shape of the RPCs can also be seen.

detect particles exiting the EMC and to help associate IFR clusters to tracks. Each side of each layer in the barrel is split into three modules in $z$. In the endcap, each layer is split vertically into two modules; each half of which contains six modules.

Figure 3.21 shows a cross-section of an RPC. RPCs detect streamers of ionising particles via capacitive readout strips. They are particularly good due to their simple, low cost construction, possibility of covering odd shapes with minimal dead space, and offer large signals and fast response so that the time resolution is $\operatorname{good}(\sim 1-2 \mathrm{~ns})$ and the front-end electronics are simple and reliable.

The RPCs consist of $2 \mathrm{~mm}$ thick plates of bakelite (phenolic polymer) that are separated by a uniform gap of $2 \mathrm{~mm}$ using insulating PVC spacers. Inside the gap there is a gas mixture of $56.7 \%$ argon, $38.8 \%$ freon-134a (1,1,1,2-tetrafluoroethane) and 4.5\% isobutane. The bulk resistivity of the bakelite sheets has been purposely tuned to $10^{11}-10^{12} \Omega \mathrm{cm}$. A graphite coating on the external surfaces achieves a surface resistivity of $100 \mathrm{k} \Omega /$ square. Of these two electrolytic surfaces, the lower electrode is grounded whilst the upper electrode is connected to a high voltage supply $(\sim 8 \mathrm{kV})$. A protective mylar film is used to 


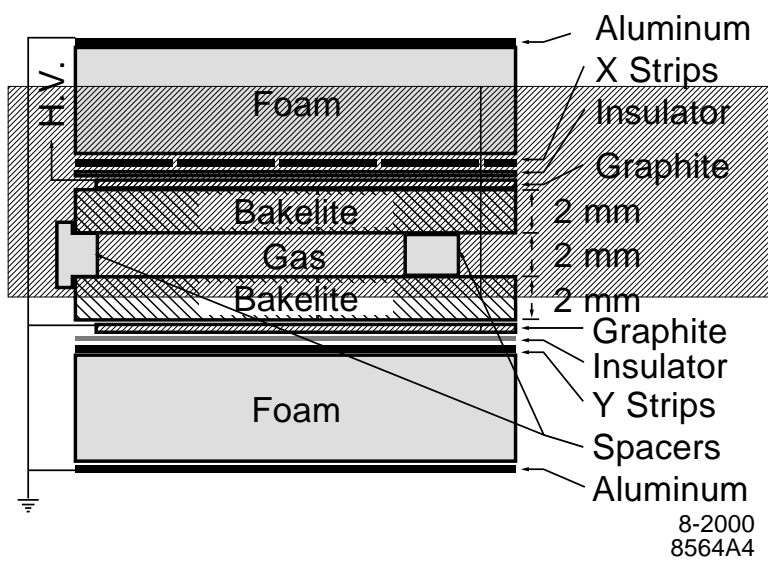

Figure 3.21: Cross-sectional view of a planar RPC.

insulate the electrodes. The bakelite surfaces facing the gap are treated with linseed oil.

An induced pulse is captured by external electrodes made of aluminium strips, labelled "X strips" and "Y strips" in Figure 3.21. Three-dimensional position information from the two orthogonal strips is obtained by making use of the finite radial thickness of the RPC. In the barrel, the pitch of the strips that measure the $z$-coordinate and $\phi$-coordinate are $38.5 \mathrm{~mm}$ and between $19.7-32.8 \mathrm{~mm}$ respectively. In the endcap, the pitch of the strips that measure the $x$-coordinate and $y$-coordinate are $38.0 \mathrm{~mm}$ and $28.3 \mathrm{~mm}$ respectively. Each front-end readout card (FEC) services data from sixteen channels, i.e. from the aluminium strips. The total number of channels is close to 53,000. The FECs select active channels and send the data to a time digitisation circuit (TDC). Even and odd numbered strips are connected to the FECs so that the failure of a card does not result in a total loss of signal. The TDC boards exploit the excellent time resolution of the RPCs. The TDC output is stored in buffers which are read out when a Level 1 accept command is received.

\subsubsection{Performance of the IFR}

The muon detection efficiency and pion misidentification probability from studies on clean control samples of muons and pions are shown in Figure 3.22. Using the selection 
corresponding to Figure 3.22, 90\% muon efficiency was achieved with a pion misidentification probability ("fake rate") between $6-8 \%$ in the momentum region $1.5-3.0 \mathrm{GeV} / \mathrm{c}$. Decays in flight contribute about $2 \%$ to the pion misidentification probability. Employing a tighter selection can halve the hadron misidentification making the sample purer, but at the expense of efficiency; the muon detection efficiency is reduced to $\sim 80 \%$.
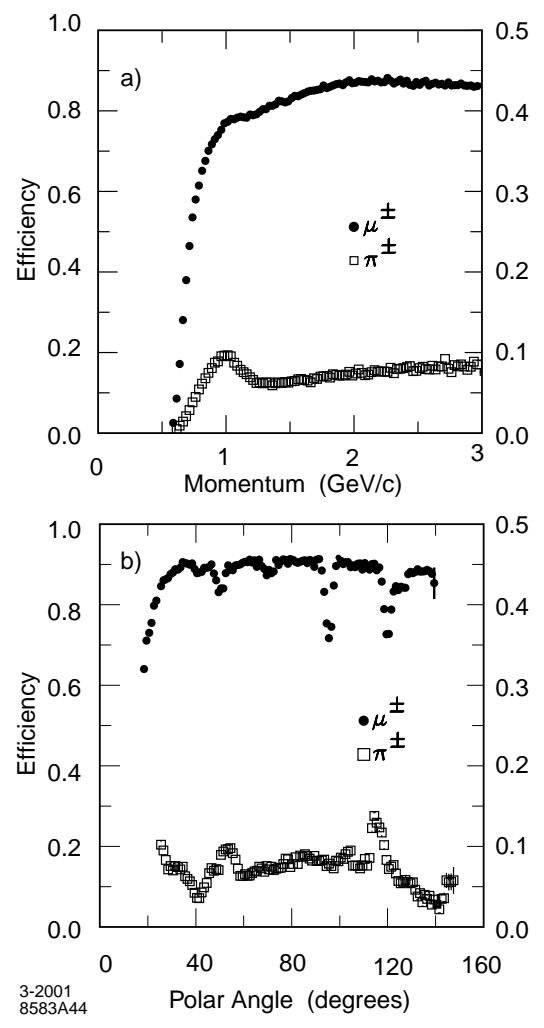

Figure 3.22: Muon efficiency (left scale) and pion misidentification probability (right scale) as a function of LAB momentum (top) and polar angle (bottom) (for $1.5<p[\mathrm{GeV} / c]<3.0$. A loose muon selection criteria was used .

$K_{L}^{0} \mathrm{~s}$ and other neutral hadrons interact in the steel of the IFR and can be identified as clusters unassociated with a charged track. According to Monte Carlo predictions, about $64 \%$ of $K_{L}^{0} \mathrm{~s}$ above $1 \mathrm{GeV} / c$ produce a cluster in the cylindrical RPC or a cluster with hits in two or more planar layers. Unassociated clusters whose angular separation is less than $0.3 \mathrm{rad}$ are combined into a composite cluster originating from showers that spread into adjacent sectors of the barrel, several sections of the end doors and/or the cylindrical RPC. Multiple clusters from large fluctuations in hadronic showers are also combined by 
this procedure. Although the direction of the neutral hadron can be determined using the event vertex and the centroid of the neutral cluster, no information on the cluster energy is obtainable.

A significant fraction of hadrons interact before reaching the IFR. Therefore, information from the EMC and the cylindrical RPCs is combined with IFR cluster information by matching the production angles. A good match (association) is obtained if the $\chi^{2}$ probability of the production angle match between neutral showers in the EMC and neutral hadrons detected in the IFR is greater than $1 \%$.

The angular resolution of the neutral hadron cluster can be estimated using a sample of $K_{L}^{0} \mathrm{~s}$ produced in the reaction $e^{+} e^{-} \rightarrow \phi \gamma \rightarrow K_{L}^{0} K_{S}^{0} \gamma$. The $K_{L}^{0}$ direction is inferred from the missing momentum calculated from the missing particles in the final state. The angular resolution derived from the IFR cluster information is found to be $\sim 60 \mathrm{mrad}$, whilst for $K_{L}^{0} \mathrm{~s}$ interacting in the EMC it is about a factor of two better.

A weekly estimate of the IFR efficiency is made using cosmic rays and colliding beam data. The muon detection efficiency has been getting progressively worse as a function of time due to an unexpected degradation of the RPCs, Figure 3.23. There are already a significant number of RPCs that have an efficiency of less than $10 \%$. Such RPCs are classified as "dead". A number of hypotheses exist as to why the RPCs are degrading so rapidly. The leading hypothesis attributes the blame to the chemistry of the linseed oil on the inside of the RPCs when exposed to an electric field. Droplets in the oil are visible and it is thought that these may be causing sparking and dark currents. Another, less popular, hypothesis blames gas flow problems.

A temporary remedy in place for most of $B A B A R$ Run 2 was to run the chambers in the opposite polarity to normal, at high voltage $(500 \mathrm{kV})$ and whilst flushing it with pure argon gas. Although this worked reasonably well for the chambers still active, it is not a satisfactory long term solution. The RPCs in the IFR endcap were replaced during the summer 2002 shutdown. It is planned to replace the RPCs in the entire barrel during a 

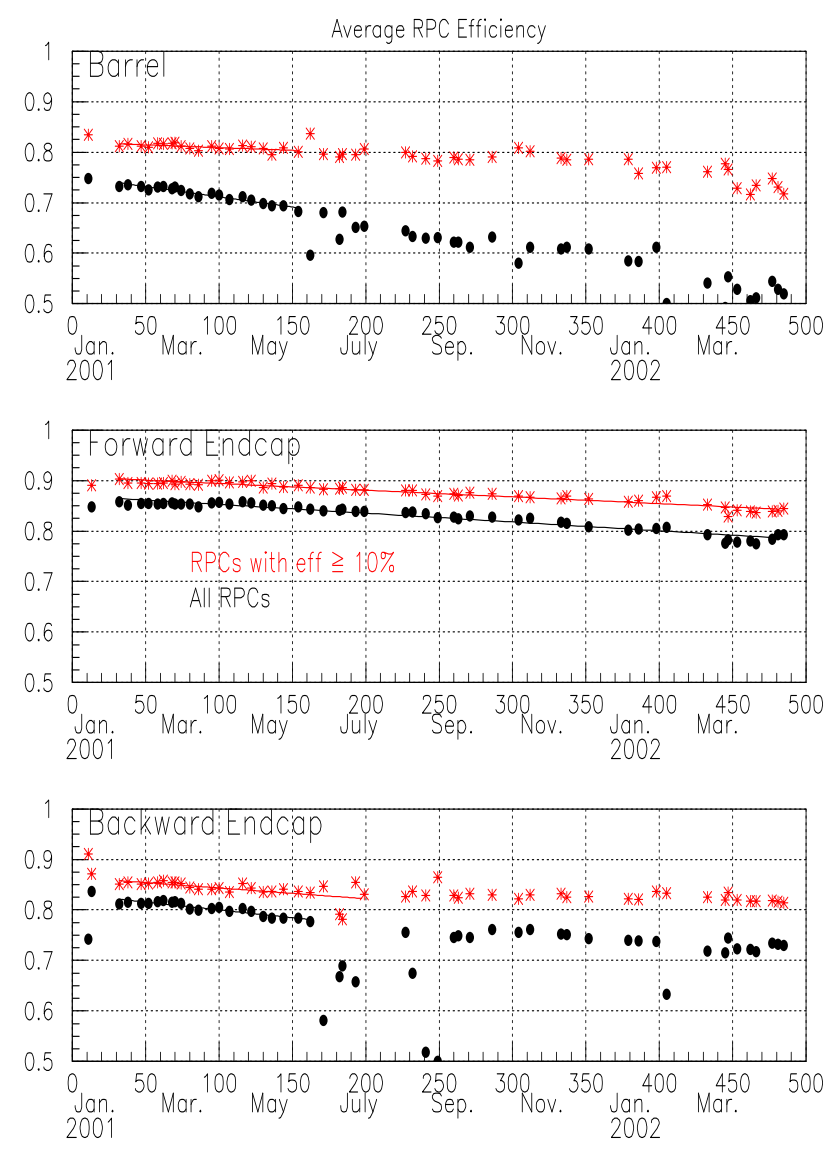

Figure 3.23: Degradation of the average RPC efficiency as a function of time for all chambers (black) and only active chambers, i.e. efficiency $\geqslant 10 \%$ (red).

long shutdown in 2004.

\subsubsection{Trigger}

The aim of the trigger system is to select the events of interest with a high, stable and wellunderstood efficiency, whilst at the same time reject background events and reduce the flow of information resulting from the very high bunch-crossing rate produced by PEP-II to a manageable event rate $(<120 \mathrm{~Hz})$ for logging and storage. At design luminosity, 
beam-induced background rates are $\sim 20 \mathrm{kHz}$ each for one or more tracks in the DCH with $p_{T}>120 \mathrm{MeV} / c$ or at least one EMC cluster whose energy is greater than $100 \mathrm{MeV}$.

The BABAR trigger operates in two stages. Firstly, the Level 1 (L1) trigger is implemented in hardware and, secondly, the Level 3 (L3) trigger is implemented in the software and operates after all the data from an event have been assembled. The total trigger efficiency is required to be in excess of $99 \%$ for all $B \bar{B}$ events and at least $95 \%$ for continuum events. Other event types can have less stringent requirements. For example, $e^{+} e^{-} \rightarrow$ $\tau^{+} \tau^{-}$events should have a trigger efficiency in the range $90-95 \%$, depending on the decay channel. The maximum allowed dead time for the trigger system is $1 \%$.

\subsubsection{Level $1(\mathrm{~L} 1)$ trigger}

The $\mathrm{L} 1$ trigger decision is based on charged tracks in the $\mathrm{DCH}$ above a preset transverse momentum, showers in the EMC and tracks detected in the IFR. It is implemented in the hardware. The part of the $\mathrm{L} 1$ trigger responsible for the $\mathrm{DCH}$ decision is called the drift chamber trigger (DCT), and that responsible for the EMC decision is called the electromagnetic calorimeter trigger (EMT). The instrumented flux return trigger (IFT) is used for triggering $\mu^{+} \mu^{-}$and cosmic rays, mostly for diagnostic purposes. All three provide input to the global trigger (GLT). Primitive trigger objects from the three triggers are sent to be combined by the GLT every $134 \mathrm{~ns}$. Events are accepted typically $\sim 1 \mathrm{kHz}$, but up to a maximum rate of $2 \mathrm{kHz}$ corresponding to the limit set by the DAQ. The GLT processes all trigger primitives to form specific triggers. Triggers are produced with a fixed latency window of 11-12 $\mu$ s after the $e^{+} e^{-}$collision and are delivered by the GLT to the fast control and timing system (FCTS). If a valid trigger remains, the FCTS issues a Level 1 accept command to initiate event readout. Efficiency studies can be done using data persisted from each trigger decision; some events are kept even if the L1 trigger lines have not been satisfied for efficiency studies. These events are known as L1 pass through events. 
The DCT primitives are defined in Table 3.4. Three types of trigger board comprise the DCT:

$\diamond$ Track Segment Finder (TSF): Looks for sets of contiguous DCH hits in a group of neighbouring cells ("pivot group").

$\diamond$ Binary Link Tracker (BLT): Links track segments to make long or short tracks. Long tracks have to reach DCH superlayer 10 and short tracks must reach DCH superlayer 5 .

$\diamond \boldsymbol{p}_{T}$ Discriminator (PTD): Uses detailed $\phi$ information on track segments found in axial superlayers to determine whether the segments are consistent with a track having a $p_{T}$ above a threshold value of $800 \mathrm{MeV} / c$.

\begin{tabular}{|c||c|c|c|}
\hline & Description & Origin & Threshold \\
\hline \hline$B$ & Short track reaching DCH superlayer 5. & BLT & $120 \mathrm{MeV} / c$ \\
$A$ & Long track reaching DCH superlayer 10. & BLT & $180 \mathrm{MeV} / c$ \\
$A^{\prime}$ & High $p_{T}$ track. & PTD & $800 \mathrm{MeV} / c$ \\
\hline
\end{tabular}

Table 3.4: Trigger primitives for the DCT. The thresholds are adjustable; typical values are given.

The EMT primitives are defined in Table 3.5. ADC counts (related to energies) are received from the EMC UPCs by the EMT. The endcap is divided into 40 towers, each forming a wedge in $\phi$ containing 19-22 crystals. For each tower, all crystal energies above a threshold of $20 \mathrm{MeV}$ are summed with those from its immediate neighbours and sent to the EMT with a "clock-4" tick (269 ns). A simple feature extraction (FEX) algorithm is applied to the resulting waveform to find the peak. The trigger processor boards (TPBs) determine the energies in the $40 \phi$ sectors, summing over various ranges in $\theta$, and their energies are compared with the thresholds of the trigger primitives (Table 3.5). An estimate of the time of the energy deposition and correction for the timing jitter are applied before transmitting the result to the GLT. 


\begin{tabular}{|c||c|c|}
\hline & Description & Threshold \\
\hline \hline$M$ & Minimum ionising cluster (all $\theta$ ). & $100 \mathrm{MeV}$ \\
$G$ & Intermediate energy cluster (all $\theta$ ). & $250 \mathrm{MeV}$ \\
$E$ & Cluster from high energy electron/photon (all $\theta$ ). & $700 \mathrm{MeV}$ \\
$X$ & Cluster from minimum ionising particle in forward endcap. & $100 \mathrm{MeV}$ \\
$Y$ & Cluster from high energy electron/photon in backward barrel. & $1 \mathrm{GeV}$ \\
\hline
\end{tabular}

Table 3.5: Trigger primitives for the EMT, all of which originate from the TPBS. The thresholds are adjustable; typical values are given.

The L1 trigger is designed so that the EMT and DCT are orthogonal. This is done so that the EMT efficiency can be determined from events that pass DCT-only trigger lines and vice versa. For $B \bar{B}$ events, both the EMT and DCT efficiencies are over $99 \%$ and the combined efficiency is greater than $99.9 \%$. High multiplicity multihadronic event topologies are prioritised by the 24 GLT trigger lines; more common calibration events, such as Bhabhas, are prescaled in order to reduce the trigger rate.

\subsubsection{Level $3(\mathrm{L3})$ trigger}

The L3 trigger is implemented in the software run on the online computer farm and the data are distributed such that each node processes one event at a time. The software comprises event reconstruction and classification, a set of event selection filters and monitoring. It is designed to refine and augment the $\mathrm{L} 1$ selection methods so that $2 \mathrm{kHz}$ input rate from the $\mathrm{L} 1$ trigger is reduced to a rate of around $100 \mathrm{~Hz}$. As information from the whole event is available, more sophisticated algorithms can be used than can be in the L1 trigger. Not only can timing information be used to reject background from other beam crossings, but also beam background can be reduced by examining track impact parameters and rejecting events not originating from the primary vertex.

The pattern recognition for the L3 DCH-based trigger, L3Dch, is done using a look-uptable and the L3 EMC-based trigger, L3Emc, identifies energy clusters with a sensitivity 
sufficient for finding minimum ionising particles. L3 trigger lines can be prescaled to keep the rate under control. The angular distributions of some calibration events, such as Bhabhas and two photon events, are prescaled so as to flatten. Some events are kept even though the L3 trigger lines are not satisfied in order to calculate efficiencies. Such events are known as $L 3$ pass through events.

\subsubsection{Online computing and data acquisition (DAQ)}

The BABAR online computing system comprises the DAQ chain from the front-end electronics (FEE), through the embedded processes in the DAQ system and the L3 trigger to the logging of the event. The infrastructure of the BABAR DAQ system is shown in Figure 3.24. Data are passed from the FEE of each subsystem to VME dataflow readout modules (ROMs) through optical fibres. The ROMs contain system-dependent firmware to carry out higher level feature extraction (FEX) which minimise backgrounds and noise. If a Level 1 accept is issued by the fast control and timing system (FCTS) the data are passed to the L3 trigger. Events passing L3 are written to a temporary (XTC) file, until they are processed by the online prompt reconstruction (OPR) code which is run on a dedicated Linux farm. The data are written to the event store by the OPR code. Currently the data are stored in two formats: in the Objectivity object-oriented database [92] and also in a ROOT-based I/O format called Kanga [93]. Users can access the data in either format within the software Framework.

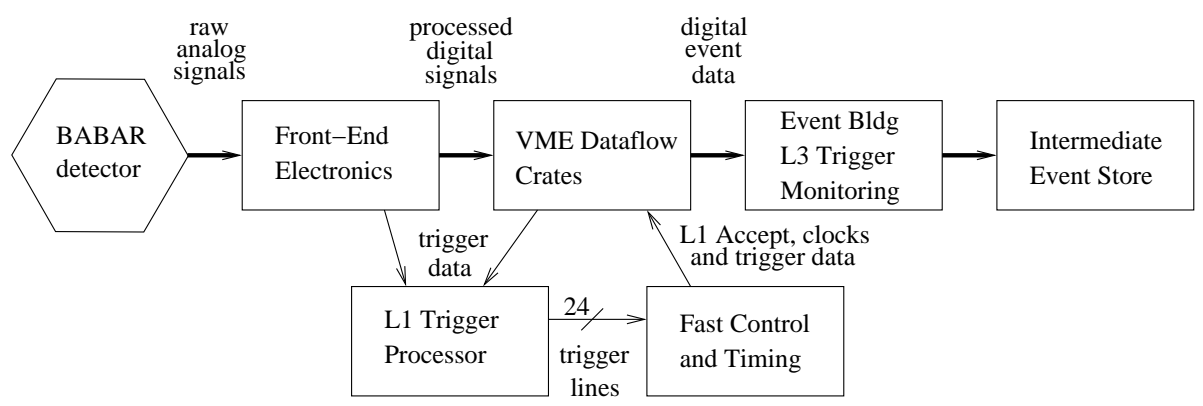

Figure 3.24: Schematic diagram of the BABAR DAQ system. 


\section{4 \\ The BABAR software, analysis tools \& data quality monitoring}

\subsection{Overview}

The BABAR software is highly complex and features simulation, reconstruction, particle identification, tagging, fitting and other analysis tools that complete the software chain. Although a detailed description of the BABAR computing environment can be found in [52], it is a rapidly evolving system that has come a long way since that publication. Updates to the computing system are, however, regularly publicised [94]. In this section a brief description of the key components relevant to this analysis is discussed.

In the past the programming language of choice for most high energy physics experiments was Fortran. BABAR was, however, the first major physics experiment to use $\mathrm{C}++[95,96]$, although others have since begun this endeavour too. Such a language allows for an object-oriented (OO) approach to software design. Object-oriented programming is particularly good for large complicated projects where code is written by 
many people. This is because analysis of a problem can be broken up into smaller parts which can be developed and tested separately. Such an approach makes for more efficient programming. $\mathrm{C}++$ is not the only language that allows $\mathrm{OO}$ to be utilised and if the choice of language were to be made again today other languages, such as Java, would also be strong candidates.

In BABAR there is a common Framework that supports the simulation, reconstruction and analysis software. Software is introduced as a package that does a specific job. The releases keep the software up-to-date so that improvements, fixes and new packages can be introduced to the users systematically. A central repository, CVS [97], keeps track of co-working versions. Data are stored in the Objectivity database [92] or as Kanga streams [93] and a global shared file system, AFS [98], is used. Data are skimmed from the database and can be stored at remote sites. Analyses can then be done locally.

\subsection{The Framework}

The Framework is the infrastructure for BABAR software. It has a "top-down" structure so that users can produce their own low-level algorithms for their particular analyses independently. The Framework is implemented as a class library. Not only does it describe the objects, but also their interactions with each other in a domain-specific manner.

Any analysis code written to interface with the Framework must be written as a welldefined module. A module is a $\mathrm{C}++$ class and the user creates an object of the module class type that is registered and then used in the execution of applications. Each module inherits from the AppModule class. A set of modules that are to be run together are organised into sequences and then into a path. The path is a complete executable.

The tool command language ( $\mathrm{tcl}$ ) [99] is used to change variables which define, enable and disable sequences of modules at run-time. Code does not therefore have to be recompiled and relinked each time. One executable binary image can therefore be used for a 
wide variety of purposes.

\subsection{Reconstruction}

Reconstructing particle candidates from individual hits in detector subsystems is done in two stages. Firstly, the reconstruction of individual tracks and photons is performed in the online prompt reconstruction (OPR) stage. This involves running the $\mathrm{E} \perp \mathrm{f}[100]$ software package over the files output from the DAQ system. The output of Elf is stored in a number of formats in an object-oriented database. The second stage is the reconstruction of composite particles. Such composites are not directly detected but are inferred by reconstructing their decay products. This stage is performed by the Beta [101] software package and a large quantity of derived software that is analysis specific.

\subsubsection{Available lists of charged tracks and neutral candidates}

Tracking algorithms are used by Elf in OPR to find charged tracks. The reconstructed tracks are categorised into different charged track lists that are stored in the micro database so that they can be accessed in subsequent analyses. Similarly, the bumps and clusters detected by the EMC are categorised by Elf into neutrals lists, depending on whether they have charged tracks associated with them or not. The lists of charged and neutral objects available in the micro database are defined in Table 4.1. All charged tracks in the micro lists are assigned a default mass based on a pion hypothesis, i.e. a mass of $139.57 \mathrm{MeV} / \mathrm{c}^{2}$.

\subsubsection{Reconstruction of composite candidates}

The presence of many particles relies on their being inferred from their decay products. This might arise if the particles are short-lived so that their tracks are not detected or are neutral and so do not leave tracks. A set of modules in the Beta analysis framework exist 
Chapter 4. The BABAR software, analysis tools \& data quality monitoring

\begin{tabular}{|c|c|}
\hline List name & Description \\
\hline ChargedTracks & $\begin{array}{c}\text { All charged tracks in } \\
\text { the SVT and/or DCH, } \\
\text { using pion mass hypothesis }\end{array}$ \\
\hline GoodTracks VeryLoose & $\begin{array}{c}\text { ChargedTracks with: } \\
\text { Momentum }<10 \mathrm{GeV} / c \\
\text { DOCA in } x y \text {-plane }<1.5 \mathrm{~cm} \\
\text { DOCA in } z<10 \mathrm{~cm}\end{array}$ \\
\hline GoodTracksLoose & $\begin{array}{c}\text { GoodTracks VeryLoose with: } \\
\qquad p_{T}>0.1 \mathrm{GeV} / c \\
\text { Number of } \mathrm{DCH} \text { hits } \geqslant 12\end{array}$ \\
\hline GoodTracksTight & $\begin{array}{c}\text { GoodTracksLoose with: } \\
\text { DOCA in } x y \text {-plane }<1 \mathrm{~cm} \\
\text { DOCA in } z<3 \mathrm{~cm}\end{array}$ \\
\hline CalorNeutral & $\begin{array}{l}\text { Single EMC bumps (including } \\
\text { single bump clusters) that } \\
\text { are not matched to any track }\end{array}$ \\
\hline CalorClusterNeutral & $\begin{array}{c}\text { Clusters of crystals } \\
\text { containing one or more } \\
\text { bumps }\end{array}$ \\
\hline GoodPhotonLoose & $\begin{array}{c}\text { CalorNeutral objects with: } \\
\text { Raw energy }>0.1 \mathrm{GeV} \\
\text { Number crystals } \geqslant 4 \\
\text { Cluster LAT }<0.8\end{array}$ \\
\hline
\end{tabular}

Table 4.1: Lists of charged tracks and neutral calorimeter objects produced in $O P R$ and stored in the BABAR micro database. DOCA abbreviates distance of closest approach.

to reconstruct composite particles from the lists in Table 4.1. The set of tools responsible for making lists of composite candidates is called CompositionTools [102].

The first step in the composition sequence is to calculate the invariant mass by 4-momenta 
addition for each possible combination of daughter comprising it:

$$
M^{\mathrm{inv}}=\sqrt{\left(\sum_{i} E_{i}\right)^{2}-\left(\sum_{i} \vec{p}_{i}\right)^{2}},
$$

where $M^{\text {inv }}$ is the invariant mass, and $E_{i}$ and $\vec{p}_{i}$ are the energy and 3-momenta of the $i^{\text {th }}$ daughter candidate respectively. If the reconstruction gets it right, a peak in the invariant mass spectrum is seen at the composite's mass. The width gives a measure of the resolution and the peak usually sits on a significant background due to combinatorics resulting from the algorithm.

\subsubsection{Vertexing and constraints}

A composite particle whose daughters are charged tracks can be vertexed to a common point by extrapolation, which uses a helix approximation to model the particle's trajectory. Beta allows for either geometric and/or kinematic fits to be performed. In the geometric fit, all the tracks are required to extrapolate back to the same point in space. Performing a kinematic fit demands that the total momentum be conserved at the decay vertex. By default, both geometric and kinematic fits are performed using an iterative $\chi^{2}$ minimisation technique. During fitting, the measured momenta of the daughter tracks are adjusted within their measurement errors. This can result in a slight change in the invariant mass of the reconstructed composite candidate. Sometimes the fits fail to converge and the composite particle's invariant mass is assigned by 4-momenta addition of the daughter candidates.

Detector resolution effects can be reduced by imposing constraints on the fit. Consider the case when a daughter of a composite particle is itself a composite. Constraining the mass of this daughter to the PDG mass when reconstructing the mother particle improves the resolution. This is particularly effective when the granddaughter particles are neutral, for example $\pi^{0} \rightarrow \gamma \gamma$; the photons do not leave tracks that can be extrapolated back to the decay vertex. 
Chapter 4. The BABAR software, analysis tools \& data quality monitoring

\subsubsection{Available lists of composite candidates}

The CompositionTools package [102] is a toolkit for use in production and analysis of BABAR data. CompositionTools is used to produce lists of composite BtaCandidates describing a given decay reaction. It uses existing lists of BtaCandidates, for example ChargedTracks and CalorNeutral, as well as other composite BtaCandidates to make new lists containing composites made from particles sharing some common criteria. The resulting BtaCandidates are then decay trees and geometric and kinematic constraints can be applied to the tree. These composite BtaCandidates are decay trees which combine tracking, neutral clusters, PID and vertexing/kinematic fitting.

Although there are many composite lists available, particularly important to this analysis is the KsDefault list. Its basis is the KsLoose list, which is a list of neutral composites (i.e. $K_{S}^{0}$ candidates) that contain associations to pairs of oppositely charged tracks from the ChargedTracks list, whose unfi tted mass is between $300-700 \mathrm{MeV} / \mathrm{c}^{2}$. The tracks are fitted, requiring them to originate from a common vertex, which changes their momenta a little. The advantage of fitting the tracks is that, by using the fitted track momenta, a better resolution for the composite's momentum is obtained. The KsDefault list is made with members of the KsLoose list whose $f$ tted mass is within $25 \mathrm{MeV} / \mathrm{c}^{2}$ of the PDG value, $m_{K_{S}^{0}}^{\mathrm{PDG}}=497.672 \pm 0.031 \mathrm{MeV} / c^{2}$ [39]. Another example is the piOAllLoose list which makes combinatorics for all $\pi^{0} \mathrm{~s}$ using the CalorNeutral list as input.

\subsubsection{Particle identification: PID selectors}

There are a number of selectors provided by the PID group to associate a charged track to particular particle type. In the case of electrons, information such as $\mathrm{d} E / \mathrm{d} x, E / p$ and EMC observables such as cluster/shower shapes are used (e.g. LAT and the Zernike moments such as Zernike-42, $A_{42}$ ); for muons, a typical signature is a small energy deposit 
in the EMC along with some number of IFR layers hit and that penetration into the IFR is consistent with a minimum ionising particle; for pions and kaons, $\mathrm{d} E / \mathrm{d} x$ information is used, particularly at lower momentum, but for tracks over approximately $700 \mathrm{MeV} / c$ the DIRC is the main source of PID information. The following references describe the original algorithms and performances of the electron [103], muon [104], pion [105] and kaon [106] selectors.

At present there are three methods used to make selectors: cut-based (Micro), likelihood ( $\mathrm{LH})$ and neural net (NN). Each selector has a number of criteria which allows the user to vary how loose or tight they want to make the selector they use depending on the purpose. In summary, Table 4.2 lists a number of selectors that are relevant to this analysis. The criteria chosen by the analyst are usually a compromise between the reduction in signal efficiency and the efficiency of the selector to get the selection correct with low misidentification probabilities. The PID group produce ntuples using the BetaPidCalib package [107]. PID tables [108] are then produced by the PID group from the BetaPidCalib ntuples which provide efficiencies and corresponding uncertainties in both real data and Monte Carlo data so that relative efficiencies can be computed to correct the Monte Carlo data to the real data ("efficiency corrections") for the various running periods of BABAR. This is necessary since imperfect detector simulation may mean that the selector performance differs between real data and Monte Carlo data; typically the efficiencies are overestimated in the Monte Carlo, perhaps due to degradation/faults during the data-taking periods that affect the real data but the simulation may not know about ${ }^{1}$. The efficiencies (and relative efficiencies) are a function of a track's 3-momentum $(\vec{p})$ and so the tables are binned in momentum $(p)$, polar angle $(\theta)$ and azimuthal angle $(\phi)$. By convention, quantities with reference to the laboratory frame are used to produce the tables. By default the PID tables are made with GoodTracksLoose tracks for electrons and muons, and ChargedTracks for pions and kaons. However, pions and kaons are selected from $D^{*}$ decays using only kinematic cuts. In order to achieve a reasonable pu-

\footnotetext{
${ }^{1}$ The Monte Carlo is updated from the conditions database with a "snapshot" of the detector conditions every month or so.
} 
rity, these cuts are quite tight. No matter which kind of track list is used as input, in the end only tracks with a good quality will survive the cuts. Further studies of the effect of changing the input track list with which tables are made might be a refinement to an analysis but are not necessarily critical, at least in a PID-weighting scheme.

To use the PID tables, there is a PID-killing module [108] that can be run before the user's analysis module to perform the procedure. Afterwards, the analyst then has a pre-PIDkilled set of Monte Carlo to work with. Another approach to using the PID-killing is to read in the PID tables directly and devise weights with which the Monte Carlo can be reweighted in order to produce any necessary correction.

\subsection{Simulation}

Monte Carlo simulated events are crucial to the vast majority of BABAR analyses. Physics events are generated and passed through a detector simulation, reconstruction and analysis code at which point they are comparable to the real data detected at the experiment. In order to achieve such realism in the simulation, the physics processes must be well modelled as must the $B A B A R$ detector itself, including the specifics of the materials and geometries comprising it. In BABAR the Monte Carlo simulation and reconstruction proceeds in four well-defined steps: event generation, detector material simulation (BOGUS), detector response simulation (SimApp) and reconstruction (BEAR). A number of software packages are in place to handle these essential aspects of the simulation. A summary of these packages is given below and a detailed description of the BABAR simulation can be found in [109].

Monte Carlo production is grouped into phases ( $\mathrm{SP} x$ ) based on a particular basis of releases of BABAR software. Most importantly for this analysis is the SP5 production which is based on Release-12. It began in January 2003 and is currently ongoing. 
Chapter 4. The BABAR software, analysis tools \& data quality monitoring

\begin{tabular}{|c|c|}
\hline PID selector & Description \\
\hline eMicroTight & $\begin{array}{c}500 \leqslant \mathrm{~d} E / \mathrm{d} x[\mathrm{MeV} / \mathrm{cm}] \leqslant 1000 \\
\left|A_{42}\right|<10 \\
0.75<E_{\text {cal }} / p<1.3 \\
\mathrm{LAT}<0.6\end{array}$ \\
\hline muMicroTight & $\begin{array}{c}0.05<E_{\text {cal }}[\mathrm{GeV}]<0.4 \\
N_{L} \geqslant 2 \\
\Delta \lambda<1 \\
\lambda>2.2 \\
\chi_{\text {trk }}^{2}<5 \\
\chi_{\text {fit }}^{2}<3 \\
T_{C}>0.3 \\
\bar{m}<8 \\
\sigma_{m}<4\end{array}$ \\
\hline piLHVeryTight & $\begin{array}{c}\text { likeKvsPi }>0.9 \\
\text { likeKvsPro }>0.20 \\
(p<0.40 \text { or } \text { not } \text { iselectron }) \\
\text { not } \text { ismuon }\end{array}$ \\
\hline
\end{tabular}

Table 4.2: Definitions of some of the many PID selectors available. eMicroTight: $\mathrm{d} E / \mathrm{d} x$ is the energy loss of the track in the material; $E_{\text {cal }}$ is the energy deposited in the EMC; $\left|A_{42}\right|$ is the Zernike-42 moment; $p$ is the track momentum (LAB); LAT is the lateral shower shape variable. muMicroTight: $N_{L}$ is the number of IFR hits; $\Delta \lambda \equiv \lambda_{\exp }-\lambda$ is the difference between the expected number of interaction lengths $\left(\lambda_{\exp }\right)$ which the track is expected to traverse in the BABAR detector under the muon hypothesis and the actual number of interaction lengths $(\lambda)$ traversed; $\chi_{\text {trk }}^{2}$ is the $\chi^{2} /$ dof of the IFR hit strips in the cluster with respect to the track extrapolation; $\chi_{\mathrm{fit}}^{2}$ is the $\chi^{2} /$ dof of the IFR hit strips with respect to a $3^{\text {rd }}$ order polynomial fit to the cluster; $T_{C}$ is the continuity of the IFR track (for $0.3<\theta<1$ tracks); $\bar{m}$ is the average multiplicity of hit strips per layer and $\sigma_{m}$ is the standard deviation of $m$. piLHVeryTight: likeKvsPi and likeKvsP ro are the kaon likelihood/pion likelihood and kaon likelihood/proton likelihood respectively; iselectron is a pass of the LH electron selector; ismuon is a pass of the very tight muon selector. These selectors are used in the $\tau^{-} \rightarrow K_{S}^{0} \pi^{-} \nu_{\tau}$ analysis. 


\subsubsection{Event generators}

The EvtGen package [110] generates many of the physics events in BABAR, particularly $B \bar{B}$ events. A file that gives the decay channels of interest along with the probability of each decay is input into the event generator. To achieve the correct angular distributions, the helicity states of the particles may too be input. For a given random seed, kinematically correct distributions are generated; the output of EvtGen is a set of 4-vectors and decay vertices for the decay products. Mixing and $C P$ violation can also be accounted for and parameters, such as polarisations of decay products and $C P$ violating phases, can be specified by users in the decay tables.

A number of other generators exist within the BABAR Framework and work in a similar manner. Particularly important for $\tau$ decays are the KoralB [111] and KK2f [112] generators, the latter of which is expected to be an improvement over the former due to its ability to handle higher order physics processes. Both KoralB and KK2f use the TAUOLA library for $\tau$ lepton decay [113]. Pre-SP5, only KoralB was used for generic $e^{+} e^{-} \rightarrow \tau^{+} \tau^{-}$Monte Carlo production. KK2 $\mathrm{f}$ is used as the principal generator of $\tau^{+} \tau^{-}$ events in SP5.

Further information about the generators and their interface to the Framework can be found in [114].

\subsubsection{BOGUS: detector simulation}

BOGUS (BABAR-Object-oriented-GEANT 4-based-Unified-Simulation) is the successor of BBsim which was used until 2001 (pre-SP4) for Monte Carlo production in BABAR. Whilst BBsim was a GEANT3-based program [115] written in Fortran, BOGUS uses the GEANT 4 simulation toolkit [116]. BOGUS, like GEANT 4, has an OO design and is written in $\mathrm{C}++$. However, GEANT 4 is not BABAR specific; it is a program by an international community of physicists with a large number of applications. 
Chapter 4. The BABAR software, analysis tools \& data quality monitoring

The GEANT 4 toolkit is designed to simulate the passage of particles through matter. Its application areas include high energy physics and nuclear experiments, medical, accelerator and space physics studies. It provides a complete set of tools for all the domains of detector simulation: geometry; tracking; detector response; run; event and track management; visualisation and a user interface [117]. An abundant set of physics processes handle the diverse interactions of particles with matter across a wide energy range. In addition, a large set of utilities, including a powerful set of random number generators, physics units and constants, Particle Data Group compliant particle management, as well as interfaces to event generators and to object-oriented databases, complete the toolkit.

BOGUS is essentially GEANT 4 applied to BABAR physics and the executable that is run is called BgsApp. This means that BOGUS has the detailed geometries of each subsystem of the BABAR detector, i.e. the specific materials, dimensions, positions and orientations. BOGUS steps charged and neutral particles through the detector and simulates the full variety of interactions and decays that each particle species may undergo. Information about tracks in sensitive components are called GTracks which are registered and stored in the database. Single interactions of a simulated particle with the detector, whether it is some charge deposited at a DCH wire, or an energy deposit in a calorimeter crystal, are known as GHits.

Tracking approximations are used in the simulation. Whilst minimum energy cuts (in units of energy) were used in BBsim, range cuts (in units of length) are used in BOGUS. This is a cut on the range of a particle's secondary. In both simulations, when a particle is prepared for a step, if its energy (BBsim) or expected range (BOGUS) is less than the cut value it is not tracked. All of its remaining energy is deposited at that place.

BOGUS also uses the GEANT 4 physics models to describe the decays of long-lived particles such as kaons, including $K_{S}^{0}$.

A paper containing validation studies of the GEANT 4-based BABAR detector simulation was presented at CHEP 2003 [118]. Incidentally, I conducted the EMC validation study 
Chapter 4. The BABAR software, analysis tools \& data quality monitoring

presented in that paper using an SP4 sample of radiative Bhabha events to study energy deposit and electromagnetic shower (cluster) shape variables between real data and GEANT 4 Monte Carlo.

\subsubsection{SimApp: detector response simulation}

The next stage in the simulation is provided by S imApp which digitises the BOGUS output by simulating the detector electronics and applying the calibration. In the actual BABAR detector, once a particle has left a deposit of energy or charge in a readout channel, each subsystem has its own array of electronics to process this information and transmit it to the DAQ system. SimApp is designed to simulate this detector response. The SimApp package contains software implementations of the algorithms used in the electronics hardware for each of the detector subsystems. Moreover, the trigger system is simulated: if a Level 1 accept is issued, the GHits in each readout channel are processed and converted into digis. digis are also created from the real data which are stored in the raw database. Therefore, after digitising the simulated data, the output is equivalent to that from the actual detector and is ready to be passed to the reconstruction software, BEAR.

SimApp uses cyclic triggers to cater for the backgrounds that arise when taking real data. These can take the form of electronics noise and beam backgrounds. In the actual BABAR DAQ system, a Level 1 accept is issued each second which initiates all the subsystems to read out their information. It is often unlikely that there is a genuine physics event taking place in the detector at the time of readout. The digis from these cyclic events are overlaid onto the digis from the generated physics event to simulate the background hits in the detector. This background mixing allows reconstruction of background tracks and hence improving correspondence between simulation and data [119]. 
Chapter 4. The BABAR software, analysis tools \& data quality monitoring

\subsubsection{BEAR: reconstruction}

In the simulation, tracks and photons are reconstructed using the BEAR package, and the produced tracks are called GTracks. Almost identical reconstruction code is run on the digis produced by the simulation as those produced by the experiment, i.e. BEAR has much of its code in common with $\mathrm{Elf}$, the package used for real data reconstruction. This code performs the final track reconstruction and fitting, cluster reconstruction and track matching and association of tracks with Čerenkov photons. Reconstructed data are then written to the database in a number of formats. In the Monte Carlo it is possible to know exactly how a particle began its life and see how it is manifest after the detector simulation has been applied. BEAR persists the true particle types, positions, momenta and energies. This information is known as Monte Carlo truth.

\subsubsection{MOOSE: combining the simulation chain}

A recent development combines the simulation chain into one step instead of the threestep BgsApp-SimApp-BEAR procedure. This tool is called MOOSE (Monolithic ObjectOriented Simulation Executable). All of the SP4 Monte Carlo uses the three-step procedure, but for SP5 MOOSE is being used. The advantage of the new method is that intermediate data structures need not be persisted.

\subsubsection{Monte Carlo production}

In order to enhance the statistical significance it is useful to produce as much simulated data as possible. Ideally this would amount to much more than in the real data, although in practice one-to-one correspondence is more realistic. This is, of course, governed by restrictions such as CPU hours, disk space and prioritisation of resources and is a computationally challenging operation. Monte Carlo production, although organised centrally, 
Chapter 4. The BABAR software, analysis tools \& data quality monitoring

relies on many sites producing simulated events around the world, many of which are in the UK. Weekly, the simulated events are exported ("swept") to the SLAC database.

In order to achieve the best realism in the Monte Carlo as possible, additional information about the detector's state must be incorporated at the time of Monte Carlo production. This information is picked up from the conditions database. Examples of these include calibration constants that differ between calibrations and the change in the DCH operating voltage, which significantly impacts on tracking efficiency. Furthermore, background conditions can change as a result of slight changes in the PEP-II configuration. A snapshot of the detector configuration is taken each month and is loaded from the conditions database when producing that month's Monte Carlo. In SP4 production, about 2,000 events per month of specific signal decays for a host of analyses were produced along with large samples of generic $\tau^{+} \tau^{-}, B \bar{B}, q \bar{q}$ and Bhabha events. The generic samples produced correspond at least one-to-one with real data. To complete the conditions matching, the cyclic background digis overlaid on the simulated events are those recorded during the corresponding month.

\subsection{Data storage and quality assurance}

The quality of the data is assessed at numerous stages. Firstly, the Data Quality Monitor (DQM) shifter checks that the data are of physics quality during data-taking. The primary DQM responsibility is to check that all the subsystems are functioning correctly. A set of live plots are cross-checked with reference plots during each run and the DQM shifter flags the data as good or bad. The data are checked again during reprocessing (OPR) after which a decision is taken on whether the data are of sufficient quality to keep.

Two primary databases are used as event stores: Objectivity and Kanga. In the Objectivity database there are several levels of detail stored from Elf output. In decreasing order of size these are known as raw, reco, micro, nano and tag. Currently being developed is the 
mini database that fits in between reco and micro and it is expected that most analyses, that presently rely most heavily on the micro database, will convert to using the mini database. The purpose of each database is:

$\diamond$ Raw

to store the digis. These are the hits in each detector subsystem, including time information, for a given event.

$\diamond$ Reco

to store objects such as tracks and neutral clusters that have been processed and combined from several digis. In principle, each reco object should correspond to a physical particle. However, there is still a good deal of information from the specific subdetectors which is useful for detector efficiency studies.

$\diamond$ Mini

to provide information about reconstructed objects from the real data, different track mass hypotheses, Monte Carlo information about GTracks and more, in addition to the micro database equivalents. A detailed description is given in [120].

$\diamond$ Micro

to store lists of BtaCandidates, the basic reconstructed objects. Only the most important information from each subsystem is stored, for example the number of IFR layers hit by a track, the Čerenkov angle and number of photons produced by a track in the DIRC. Most current BABAR analyses are based on micro data.

\section{$\diamond$ Nano/tag}

to provide a means of fast filtering at the start of an analysis job. Contains basic information about the whole event, e.g. number of charged tracks, that can be used for a loose, faster preselection. (Doing anything other than a loose preselection at the nano level could cause problems due to subtle differences in definitions between the nano and mico databases.) Based on whether events are selected or not a tagbit 
specifying a pass or fail can be written into the tag database. During a later skim of the data, failed events can quickly be removed.

Most important for this analysis are the tag and micro databases and a detailed description of what is available in these databases can be found in [121]. In the future, the mini, which is designed to work within the new computing model, will replace the micro as the primary analysts' database [120]. In this analysis, the Beta job, which performs some vertexing, particle reconstruction and loose cuts, is run on the micro data. The quantities of interest are output into ntuples, which are essentially large tables containing the desired quantities, for example momenta and vertex positions, for the reconstructed candidates in the events. These ntuples can be produced in either PAW or ROOT format, although in ROOT they appear as "trees". This analysis uses the ROOT format which is discussed in Section 4.7 .

\subsection{TauUser analysis package}

TauUser is a Beta-based analysis package producing a common set of tools for a number of tau analyses and is documented in detail in $[122,123]$. It is currently used to produce a common set of ntuples with broad cuts. Users can run their analyses at the ntuple level using the data (real and simulated) processed by TauUser. Not only does TauUser allow the user to run interactive analyses in ROOT (or PAW) using the many useful quantities filled during processing, but also make use of reconstructed quantities agreed by the Tau/QED Analysis Working Group (AWG). For example, there is a set of reconstructed $\pi^{0}$ variables that have been studied by the AWG to provide a more suitable $\pi^{0}$ reconstruction for tau physics than the default list does. These are included with the package.

The analysis is done in three modules: 
Chapter 4. The BABAR software, analysis tools \& data quality monitoring

$\diamond$ TauNanoFilter: a fast filter which processes the nano database information.

$\diamond$ TauMain: produces the basic ntuple and makes preselection cuts.

$\diamond$ MyTauAnalysis: allows the user to edit and add their own functions which are executed in TauMain.

\subsection{Data analysis with ROOT}

The ROOT system [124] provides a set of object-oriented frameworks with all the functionality needed to handle and analyse large amounts of data in an efficient way. ROOT is written in $\mathrm{C}++$ and provides a number of shared libraries that a user can use within their programs. ROOT code can be pre-compiled in the usual way (e.g. using gcc or egs compilers) to make an executable or can be interpreted at run-time using the CINT C++ interpreter. The interpreter allows for fast prototyping of the macros since it removes the time consuming compile/link cycle.

Useful features of ROOT include: the command line interpreter (CINT); histogramming and fitting; 2D/3D graphics; I/O; collection classes; script processing and graphical user interfaces (GUIs). Advanced features include: thread support; parallel processing (PROOF); run-time type identification (RTTI) and socket and network communication.

In ROOT's predecessor, PAW [125], ntuples containing essentially arrays of numbers can just be stored. These can be manipulated using Fortran routines. In ROOT, however, whole objects can be stored in a TTree, ROOT's improved version of the ntuple. Not only can data be accessed, but also member functions of that object's type. For example, storing a TLorentzVector object allows the $\mathrm{M}()$ or Beta () method to be called in future macros which reuse code to calculate the mass or $\beta$ respectively of the 4-vector. Operators are overloaded to allow 4-vector addition and other manipulation, such as dot and cross products, to be conducted in a single line of code. Likewise boosts to other 
frames of reference can be done effortlessly with the Boost () method. This is just one example of the many ways encapsulation, inheritance and the class hierarchy structure of ROOT's OO design benefits the end user. Users can also write their own shared-object libraries that can be linked against the ROOT libraries (or loaded in the case of CINT) to do their favourite tasks over and over again if desired.

\subsection{Fitting the data}

\subsubsection{Maximum likelihood}

\subsubsection{Maximum likelihood estimation}

Maximum likelihood (ML) is used as a method of estimation. It is described in detail in [126-128]. The distribution of a set of variables (e.g. experimental measurements), $x_{i}$, can be described by a normalised probability density function (PDF), $P\left(x_{i} ; a\right)$, for a set of parameters $a$. For a data sample $\left\{x_{1}, \ldots, x_{N}\right\}$, the maximum likelihood estimator $\hat{a}$ is the value of $a$ for which the likelihood function,

$$
\mathcal{L}\left(x_{1}, \ldots, x_{N} ; a\right)=\prod_{i=1}^{N} P\left(x_{i} ; a\right),
$$

is a maximum. This is also true if $P$ is a combination of other normalised PDFs, for example a linear combination of signal and background.

In practice, it is easier to maximise the logarithm of the likelihood function,

$$
\ell=\ln \mathcal{L}=\sum_{i=1}^{N} \ln P\left(x_{i} ; a\right),
$$

which is the sum (rather than product) of the probabilities. Moreover, in many cases differentiating Eq. (4.3) and setting it to zero is the easiest way to find the maximum:

$$
\left.\frac{\mathrm{d} \ln \mathcal{L}}{\mathrm{d} a}\right|_{a=\hat{a}}=0
$$




\subsubsection{Errors on the ML estimators}

In the limit of large $N$, i.e. a large number of measurements, the probability distribution for $\hat{a}$ is Gaussian; a consequence of the central limit theorem (CLT), described, for example, in [126]. For this to be exactly true, the second derivative $\left(\mathrm{d}^{2} \ln \mathcal{L} / \mathrm{d} a^{2}\right)$ must be a constant. However, a good approximation is achieved if $\left(\mathrm{d}^{2} \ln \mathcal{L} / \mathrm{d} a^{2}\right)$ only changes a little in the range of $a$ in the vicinity of the true value $a_{0}$. Taylor expanding $\ell$ around its maximum, $\ell_{\max }$, gives:

$$
\ell=\ell_{\max }+\frac{1}{2 !} \ell^{\prime \prime}\left(\delta a^{2}\right)+\cdots,
$$

where the $\mathcal{O}\left(l^{\prime}\right)$ term is zero by Eq. (4.4). From Eq. (4.5), the log likelihood is a parabola whose maximum occurs at $a_{0}$. Using Eq. (4.3), introducing a constant, $-1 / k$, for the second derivative, and integrating and exponentiating yields:

$$
\mathcal{L}\left(x_{1}, \ldots, x_{N} ; a\right) \sim \exp \left(-\frac{\left(a-a_{0}\right)^{2}}{2 k}\right) .
$$

The likelihood function obtained from the data is Gaussian. For Gaussian $\mathcal{L}$, the best estimate of the error is obtained from

$$
\ell\left(a_{0} \pm \delta a_{0}\right)=\ell_{\max }-0.5 .
$$

\subsubsection{Extended maximum likelihood}

Extended maximum likelihoods (EMLs) are described in detail in [129]. EMLs differ from the standard MLs in that the normalisation of the PDF is allowed to vary; in the ML method the integrated PDF is always normalised to 1. EMLs are therefore useful for problems in which the number of events obtained is itself a relevant measurement. If the function is such that its size and shape can be independently varied, then the estimates given by the EML method are identical to the standard ML estimators, though care must be taken in the interpretation of the errors. However, if the function does not have this property, then EML can give better results. 
The normalisation in EML fits is related to the event yield. A Poisson distribution with mean $=$ variance $=\nu$ is used to represent the event yield. The likelihood then becomes

$$
\mathcal{L}(\nu ; a)=\frac{e^{-\nu} \nu^{N}}{N !} \prod_{i=1}^{N} P\left(x_{i} ; a\right)=\frac{e^{-\nu}}{N !} \prod_{i=1}^{N} \nu P\left(x_{i} ; a\right) .
$$

The log likelihood is:

$$
\ell(\nu ; a)=\sum_{i=1}^{N} \ln P\left(x_{i} ; a\right)-\nu+N \ln \nu,
$$

where the $N$ ! term has been dropped as it is independent of $a$. The expected number of events, $\nu$, is then a parameter in the fit. In a branching fraction analysis, this technique is particularly useful for determining the yields in different categories of events. From the fit, the extraction of the number of signal and background events based on suitable models (PDFs) of the data can be done.

\subsubsection{RooFit: an interface to Minuit}

Since 1975, Minuit [130] has become widely used as a minimisation package that searches parameter space to find the minimum of a given function. For the case of maximum likelihood fitting, the function to be minimised is $-\ell=-\ln \mathcal{L}$.

The RooF it [131,132] software package provides a user-friendly interface to the ROOT Minuit library, and also makes use of other ROOT libraries for fitting, histogram plotting, data management, etc. RooF it is capable of performing maximum likelihood and extended maximum likelihood fits, including handling of the normalisation. Numerous PDF models are provided in the tools (e.g. Gaussian, Breit-Wigner) and the user is able to create their own PDFs. Operations such as combining these PDFs (e.g. addition, multiplication, convolution) are possible and it is also possible to overlay the component PDFs comprising the combined PDF on the same plot. This is useful for looking at signal and background or DIRC PID likelihoods for example. Simultaneous fits to different samples with different PDFs that share some parameters can be made. In addition, the fit values 
can be extracted and used to make a graph with errors over the range of fitted slices of a particular quantity.

A really useful feature of RooFit is the ease with which "toy Monte Carlo" can be generated for mock studies. 


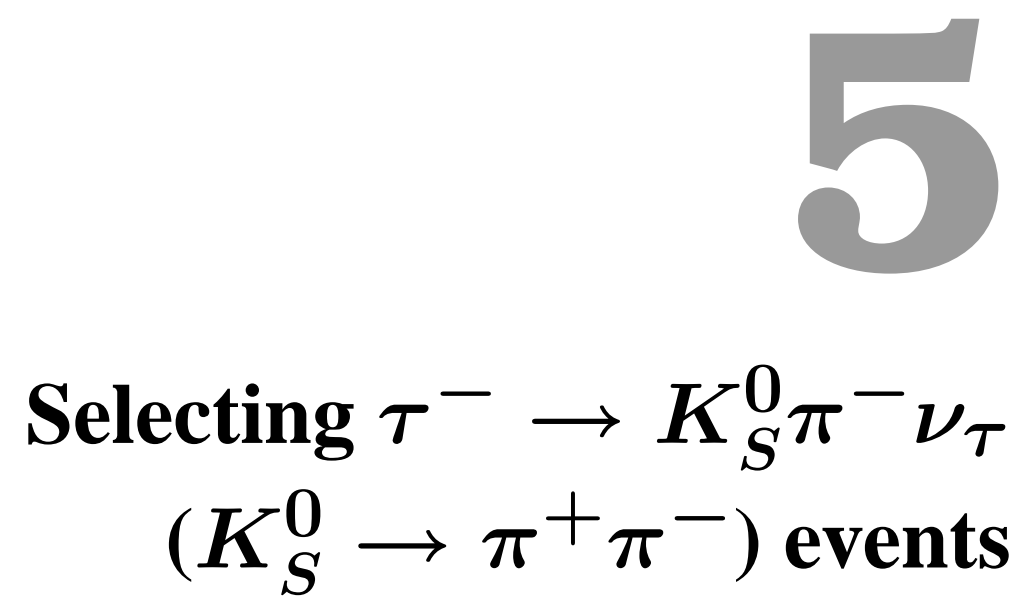

\subsection{Physics data and Monte Carlo samples}

Only data flagged as "good-runs" in the quality assurance were used to ensure that the detector was fully operational and working as expected for the collections analysed. Such data are called "physics data" as its passing the quality assurance means it is of physics quality. The physics data used in this analysis are from the Run $1+2+3$ data set processed in the 12-series. These data amount to $113.3 \mathrm{fb}^{-1}$ on-resonance and $12.1 \mathrm{fb}^{-1}$ offresonance, i.e. $125.4 \mathrm{fb}^{-1}$ in total [84]. In practice, these numbers were slightly smaller at the time of ntuple production due to a few database problems. The actual data luminosities analysed are given in Table 5.1.

The corresponding 12-series (SP5) Monte Carlo samples used in this analysis are listed in Table 5.2. For a complete set of available SP5 Monte Carlo samples see [133]. The SP5 $e^{+} e^{-} \rightarrow \tau^{+} \tau^{-}$events used were generated using the KK2f event generator [112]. 
The branching fractions used by KK2 $f$ in SP5 production are based on PDG 2002 [39] averages (not fitted values) with a unitarity constraint imposed ${ }^{1}$, and are listed explicitly in Table B.1 in Appendix B. Using these branching fractions it is possible to calculate the number of signal $\tau^{ \pm} \stackrel{K^{* \pm}}{\longrightarrow} K_{S}^{0} \pi^{ \pm} \bar{\nu}_{\tau}\left(K_{S}^{0} \rightarrow \pi^{+} \pi^{-}\right)$generated from the total number of $\tau^{+} \tau^{-}$pairs generated $\left(N_{\tau \tau}^{\text {gen }}\right)$ and the PDG branching fractions ${ }^{2}$ :

$$
\begin{aligned}
N_{\text {sig }}^{\text {gen }} & =2 \times N_{\tau \tau}^{\text {gen }} \times \mathcal{B}\left(\tau^{ \pm} \stackrel{K^{* \pm}}{\longrightarrow} K^{0} \pi^{ \pm} \bar{\nu}_{\tau}\right) \times \mathcal{B}\left(K^{0}=K_{S}^{0}\right) \times \mathcal{B}\left(K_{S}^{0} \rightarrow \pi^{+} \pi^{-}\right) \\
& =2 \times 156776000 \times 0.90 \% \times 50 \% \times 68.60 \% \\
& =9.679 \times 10^{5}
\end{aligned}
$$

The signal branching fraction has been checked at the generator level using a sample of $10 \mathrm{M}$ generic $\tau^{+} \tau^{-}$decays, of which 93 decayed with each $\tau$ decaying as $\tau^{ \pm} \stackrel{K^{* \pm}}{\longrightarrow}$ $K_{S}^{0} \pi^{ \pm} \bar{\nu}_{\tau}\left(K_{S}^{0} \rightarrow \pi^{+} \pi^{-}\right)$(i.e. "double $K^{*}$ decay") [134]. Using these data, the branching fraction is approximately $\sqrt{93 / 10^{7}}=0.305 \%$ which is consistent with the PDG 2002 value (Eq. (1.5)).

\begin{tabular}{|c||c|}
\hline Physics data & $\mathcal{L}\left(\mathrm{fb}^{-1}\right)$ \\
\hline \hline Run 1 + Run 2 (on) & 81.40 \\
Run 1 + Run 2 (off) & 9.55 \\
Run 3 (on) & 31.06 \\
Run 3 (off) & 2.39 \\
\hline TOTAL & 124.4 \\
\hline
\end{tabular}

Table 5.1: Amount of 12-series on-resonance and off-resonance physics data processed to make the TauUser ntuples.

${ }^{1}$ The unitarity constraint raises the generator value of $\mathcal{B}\left(\tau^{ \pm} \stackrel{K^{* \pm}}{\longrightarrow} K^{0} \pi^{ \pm} \bar{\nu}_{\tau}\right)$ slightly to $0.90 \%$, compared with the PDG fi tted value of $\mathcal{B}\left(\tau^{ \pm} \stackrel{K^{* \pm}}{\longrightarrow} K^{0} \pi^{ \pm} \bar{\nu}_{\tau}\right)=(0.89 \pm 0.04) \%$.

${ }^{2}$ For $\mathcal{B}\left(\tau^{ \pm} \stackrel{K^{* \pm}}{\longrightarrow} K^{0} \pi^{ \pm} \bar{\nu}_{\tau}\right)$, the value input into the generator is used (Table B.1). 
Chapter 5. Selecting $\boldsymbol{\tau}^{-} \rightarrow \boldsymbol{K}_{S}^{0} \boldsymbol{\pi}^{-} \boldsymbol{\nu}_{\boldsymbol{\tau}}\left(\boldsymbol{K}_{S}^{0} \rightarrow \boldsymbol{\pi}^{+} \boldsymbol{\pi}^{-}\right)$events

\begin{tabular}{|c||c|c|c|}
\hline Monte Carlo sample & Number of events generated & $\sigma(\mathrm{nb})$ & $\mathcal{L}\left(\mathrm{fb}^{-1}\right)$ \\
\hline \hline$\tau^{+} \tau^{-}$ & $156,776,000$ & 0.89 & 176.2 \\
$u d s$ & $299,501,000$ & 2.09 & 143.3 \\
$c \bar{c}$ & $185,828,000$ & 1.30 & 142.9 \\
$B^{0} \bar{B}^{0}$ & $245,532,000$ & 0.54 & 454.7 \\
$B^{+} B^{-}$ & $220,494,000$ & 0.54 & 408.3 \\
\hline
\end{tabular}

Table 5.2: Generated Monte Carlo samples in SP5 (12-series) production.

\subsection{Event preselection}

\subsubsection{TauUser preselection}

This analysis uses a set of ntuples produced with TauUser V00-11-03 using release analysis-14a. The amount of physics data processed by TauUser in this production is shown in Table 5.1 and of Monte Carlo data in Table 5.2. The topology of $e^{+} e^{-} \rightarrow \tau^{+} \tau^{-}$events is characterised by a pair of back-to-back narrow jets with low particle multiplicity. An event is split into two hemispheres using the thrust axis of the event created by summing the momenta of the GoodTracksVeryLoose and neutrals in the event. TauUser classifies events into topologies based on the number of GoodTracksVeryLoose in each hemisphere, i.e. the dot product of the momentum of each of the GoodTracksVeryLoose with the thrust axis is taken and which hemisphere the track is in depends on whether this result is positive or negative. Even though the event topology is defined by the number of GoodTracksVeryLoose, additional ChargedTracks are still retained in the event even if they do not overlap the GoodTracksVeryLoose list (Table 4.1). Important to this analysis are the 1-1, 1-2 and 1-3 topologies since $K_{S}^{0}$ events are characterised by a displaced vertex due to the relatively long lifetime of the $K_{S}^{0}$ and so genuine $K_{S}^{0} \rightarrow \pi^{+} \pi^{-}$events can be made from each of those topologies so long as there are the necessary number of ChargedTracks. Events in which both taus decay to a $K_{S}^{0}$ are removed by the topology requirements. Defining a "one-prong side" is necessary to tag the non-signal side of the event as a lep- 
ton, something which is done later to reduce hadronic backgrounds.

TauUser applies preselection cuts in two stages: firstly using the nano information for fast filtering and, secondly, using the micro information. A list of the TauUser preselection can be found below:

$\diamond$ Pass one of the trigger flags: L3OutDch or L3OutEmc (nano).

$\diamond$ Stream19 tagbit set (nano).

$\diamond$ Between 2 and 11 charged tracks (nano).

$\diamond$ Between 2 and 9 good tracks (nano).

$\diamond$ Between 2 and 9 charged tracks, i.e. ChargedTracks (micro).

$\diamond$ Between 2 and 8 good tracks, i.e. GoodTracksVeryLoose (micro).

$\diamond$ All topologies on (micro).

$\diamond$ Thrust $\geqslant 0.85$ (micro).

$\diamond$ Between 0 and 10 AWG $\pi^{0}$ candidates (micro).

$\diamond$ Between 0 and 20 piOAll Loose candidates (micro).

$\diamond$ Between 0 and 20 CalorNeutrals (micro).

This preselection is intentionally very loose so that it can be used to produce ntuples suitable for a number of tau analyses and not just this one. One of the key features of tau events that this preselection exploits is the low track multiplicity of tau decays. $B$ decays, which are very common at BABAR, tend to have higher track multiplicities $(\gtrsim 12)$ and so can be cut away at a very early stage. 
Chapter 5. Selecting $\boldsymbol{\tau}^{-} \rightarrow \boldsymbol{K}_{S}^{0} \pi^{-} \boldsymbol{\nu}_{\tau}\left(\boldsymbol{K}_{S}^{0} \rightarrow \boldsymbol{\pi}^{+} \boldsymbol{\pi}^{-}\right)$events

\subsubsection{Additional preselection - skim ntuple}

In addition to the TauUser preselection, which acts as a wide preselection for many analyses, further preselection requirements were imposed to make a skim of the TauUser ntuples with a view to studying the $K^{*-} \rightarrow K_{S}^{0} \pi^{-}$system. According to PDG 2002 [39], by far the two largest branching fractions of the $K_{S}^{0}$ are $\mathcal{B}\left(K_{S}^{0} \rightarrow \pi^{+} \pi^{-}\right)=(68.60 \pm$ $0.27) \%$ and $\mathcal{B}\left(K_{S}^{0} \rightarrow \pi^{0} \pi^{0}\right)=(31.40 \pm 0.27) \%$. In this analysis only $K_{S}^{0} \rightarrow \pi^{+} \pi^{-}$ events are reconstructed. Therefore, in order to vastly reduce the size of the data sample at an early stage, by reducing backgrounds whilst preserving the majority of signal events, the following additional preselection cuts were imposed:

$\diamond$ Combine 1-1, 1-2 and 1-3 topologies (based on GoodTracksVeryLoose) and discard the rest.

$\diamond N_{\text {ch }}=4$, i.e. the number of charged tracks in the event from the ChargedTracks list is exactly 4.

$\diamond N_{K_{S}^{0}}$ cands $\neq 0$, i.e. there is at least 1 candidate in the KsDefault list.

$\diamond \sum Q_{i}=0$, i.e. the net charge of all the charged tracks from the ChargedTracks list is exactly 0 .

Using these additional preselection criteria on a 500,000 event sub-sample of all SP5 generic $e^{+} e^{-} \rightarrow \tau^{+} \tau^{-}$events gives the results shown in Table 5.3. The notation $\tau^{-} \stackrel{K^{*-}}{\longrightarrow}$ $K_{S}^{0} \pi^{-} \nu_{\tau}$, where the $K^{*-}$ is explicitly included, is to emphasise the fact that in the Monte Carlo only a resonant (in fact only the $K^{*}(892)$ resonance) contribution is simulated and so all of the $\tau^{-} \rightarrow K_{S}^{0} \pi^{-} \nu_{\tau}$ events come from a $K^{*-}$ resonance. From Table 5.3 it can be seen that the efficiencies of these additional preselection cuts are around $50 \%, 50 \%$ and $70 \%$ for the 1-1, 1-2 and 1-3 topologies respectively and, at the same time, the generic $\tau$ sample is reduced by approximately $95 \%$ showing a large reduction in $\tau$ backgrounds. Moreover, these cuts also act to reduce the non- $\tau$ backgrounds which leads to a substantial reduction in the overall data sample size at the preselection stage. 
Chapter 5. Selecting $\boldsymbol{\tau}^{-} \rightarrow \boldsymbol{K}_{S}^{0} \boldsymbol{\pi}^{-} \boldsymbol{\nu}_{\boldsymbol{\tau}}\left(\boldsymbol{K}_{S}^{0} \rightarrow \boldsymbol{\pi}^{+} \boldsymbol{\pi}^{-}\right)$events

\begin{tabular}{|c||c|c|c||c|c|c|}
\hline \multicolumn{1}{|c||}{ Monte Carlo truth } & \multicolumn{3}{c||}{ Before } & \multicolumn{3}{c|}{ After } \\
\hline After TauUser & $1-1$ & $1-2$ & $1-3$ & $1-1$ & $1-2$ & $1-3$ \\
\hline$\tau^{+} \rightarrow K_{S}^{0} X^{+} \bar{\nu}_{\tau}$ & 161958 & 10587 & 64467 & 1536 & 352 & 8260 \\
\hline \multicolumn{1}{|c||}{$\tau^{+} \rightarrow K_{S}^{0} X^{+} \bar{\nu}_{\tau}\left(\geqslant 1 K_{S}^{0} \rightarrow \pi^{+} \pi^{-}\right)$} & 221 & 235 & 844 & 120 & 117 & 593 \\
$\tau^{+} \rightarrow K_{S}^{0} X^{+} \bar{\nu}_{\tau}\left(\geqslant 1 K_{S}^{0} \rightarrow \pi^{0} \pi^{0}\right)$ & 479 & 44 & 150 & 6 & 2 & 40 \\
\hline$\tau^{+} \stackrel{K^{*+}}{\longrightarrow} K_{S}^{0} \pi^{+} \bar{\nu}_{\tau}$ & 363 & 128 & 468 & 67 & 64 & 310 \\
$\tau^{+} \stackrel{K^{*+}}{\longrightarrow} K_{S}^{0} \pi^{+} \bar{\nu}_{\tau}\left(K_{S}^{0} \rightarrow \pi^{+} \pi^{-}\right)$ & 117 & 112 & 414 & 65 & 64 & 301 \\
$\tau^{+} \stackrel{K^{*+}}{\longrightarrow} K_{S}^{0} \pi^{+} \bar{\nu}_{\tau}\left(K_{S}^{0} \rightarrow \pi^{0} \pi^{0}\right)$ & 240 & 15 & 54 & 2 & 0 & 9 \\
\hline$\tau^{-} \rightarrow K_{S}^{0} X^{-} \nu_{\tau}$ & 682 & 244 & 941 & 117 & 104 & 570 \\
$\tau^{-} \rightarrow K_{S}^{0} X^{-} \nu_{\tau}\left(\geqslant 1 K_{S}^{0} \rightarrow \pi^{+} \pi^{-}\right)$ & 209 & 222 & 836 & 112 & 104 & 559 \\
$\tau^{-} \rightarrow K_{S}^{0} X^{-} \nu_{\tau}\left(\geqslant 1 K_{S}^{0} \rightarrow \pi^{0} \pi^{0}\right)$ & 473 & 26 & 125 & 8 & 1 & 26 \\
\hline$\tau^{-} \stackrel{K^{*-}}{\longrightarrow} K_{S}^{0} \pi^{-} \nu_{\tau}$ & 333 & 113 & 487 & 58 & 49 & 306 \\
$\tau^{-} \stackrel{K^{*-}}{\longrightarrow} K_{S}^{0} \pi^{-} \nu_{\tau}\left(K_{S}^{0} \rightarrow \pi^{+} \pi^{-}\right)$ & 106 & 101 & 431 & 56 & 48 & 301 \\
\hline$\tau^{-} \stackrel{K^{*-}}{\longrightarrow} K_{S}^{0} \pi^{-} \nu_{\tau}\left(K_{S}^{0} \rightarrow \pi^{0} \pi^{0}\right)$ & 224 & 12 & 56 & 2 & 1 & 5 \\
\hline
\end{tabular}

Table 5.3: Monte Carlo true decay modes using a 500,000 event sub-sample of generic $e^{+} e^{-} \rightarrow \tau^{+} \tau^{-}$events before and after the additional preselection cuts $\left(N_{\mathrm{ch}}=4, N_{K_{S}^{0}}\right.$ cands $\left.\neq 0, \sum Q_{i}=0\right)$. The initial number of events, i.e. those surviving the preselection imposed by TauUser is listed in the first row for each of the topologies: 1-1, 1-2 and 1-3. $X$ is used to represent any system of particles. Note that the $\tau^{ \pm} \rightarrow K_{S}^{0} X^{ \pm} \nu_{\tau}$ breakdown into $K_{S}^{0} \rightarrow \pi^{+} \pi^{-}$and $K_{S}^{0} \rightarrow \pi^{0} \pi^{0}$ can have overlapping events since the $X$ itself can contain additional $K_{S}^{0}$ particles, e.g. in decay modes with two $K_{S}^{0} \mathrm{~s}$. Moreover, the ultimate signal decay mode is highlighted in yellow.

\subsection{Selection procedure}

\subsection{1 $\quad K_{S}^{0} \rightarrow \pi^{+} \pi^{-}$reconstruction}

The identification of $\tau$ decays with $K_{S}^{0} \rightarrow \pi^{+} \pi^{-}$can be achieved with high efficiency and good mass resolution. For the $\tau^{-} \rightarrow \bar{K}^{0} \pi^{-} \nu_{\tau}$ channel, $50 \%$ consists of $K_{L}^{0}$ and $50 \%$ of $K_{S}^{0} . K_{L}^{0}$ decays have a signature of a large energy deposit (cluster) in the BABAR IFR without an associated track pointing to the cluster. (EMC information is also used 
to calculate the fraction of neutral hadrons interacting with the CsI in the EMC before reaching the IFR.) Due to efficiency problems with the IFR it was decided not to use the $K_{L}^{0}$ channel. For the $K_{S}^{0}$ channel, there are two decay modes: $K_{S}^{0} \rightarrow \pi^{0} \pi^{0}(\mathcal{B} \simeq 32 \%)$ and $K_{S}^{0} \rightarrow \pi^{+} \pi^{-}(\mathcal{B} \simeq 68 \%)$. Because of wanting the best possible $K_{S}^{0}$ mass resolution, only the latter is considered in this analysis.

The decay $K_{S}^{0} \rightarrow \pi^{+} \pi^{-}$is reconstructed using two oppositely charged tracks from the ChargedTracks list of all the reconstructed tracks in the event that are in the same hemisphere and overlap the KsDefault list. The KsDefault list contains pairs of oppositely charged tracks that have an invariant mass within $25 \mathrm{MeV} / c^{2}$ of the PDG value, $m_{K_{S}^{0}}^{\mathrm{PDG}}=497.672 \pm 0.031 \mathrm{MeV} / c^{2}$ [39]. This list is made by vertexing the tracks and in so doing a more precise value of the $\pi^{+} \pi^{-}$invariant mass is achieved than by merely adding the charged tracks' 4-momenta before vertexing. Some background is also removed this way.

Since the momenta of the $K_{S}^{0}$ daughters are changed by the fitting procedure they must be recalculated because, by default, the track momenta of the ChargedTracks, even if there is an overlap with the KsDefault list, are those that do not have any vertex constraint imposed. This means that whilst one benefits from the better mass resolution by using the fitted mass from the KsDefault list (i.e. because it was computed with the vertex constraint), the momenta of the daughter tracks do not correspond to the fitted candidates since the unfitted values are returned by default. To retrieve the corresponding (i.e. vertex-constrained) track momenta, the fit procedure is performed again and the refitted values retained. A three-dimensional vertex fit without kinematic constraint is used to constrain the $K_{S}^{0}$ candidate's daughters to originate from a common vertex by requiring a success status from the GeoKin vertex fitter [135]. As a consequence the $\pi^{+} \pi^{-}$invariant mass made my summing the refitted $K_{S}^{0}$ daughter momenta is identical to that obtained directly from the KsDefault list, as it should be.

Figure 5.1 shows the $K_{S}^{0}$ mass for candidates from the KsDefault list without any 
additional selection. Clearly the $K_{S}^{0}$ signal sits on a large background: the list contains a large number of non- $K_{S}^{0}$ combinations of $\pi^{+} \pi^{-}$.

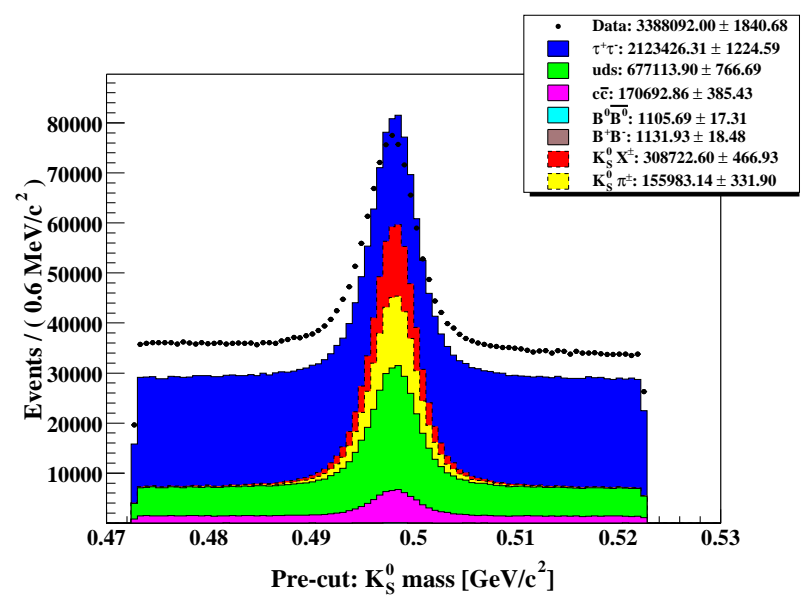

Figure 5.1: $K_{S}^{0}$ mass $\left(\pi^{+} \pi^{-}\right.$combinations) from the KsDefault list before any additional selection. Clearly the $K_{S}^{0}$ signal sits on a large background. The Monte Carlo is plotted cumulatively. $K_{S}^{0} X^{ \pm}$and $K_{S}^{0} \pi^{ \pm}$are components of $\tau^{+} \tau^{-}$.

Figure 5.2 and Figure 5.3 show the distributions of a number of quantities used to select good $K_{S}^{0} \rightarrow \pi^{+} \pi^{-}$candidates. To this end, the following quality cuts are applied:

$\diamond$ Only $1 K_{S}^{0}$ candidate in the KsDefault list.

$\diamond$ GeoKin vertex fitter successfully fits the $K_{S}^{0}$ daughters.

$\diamond \Delta^{\text {sep }}<3 \mathrm{~mm} . \Delta^{\text {sep }}$ is the separation of the $K_{S}^{0}$ daughters from each other at their respective points of closest approach.

$\diamond \chi_{\text {prob }}^{2}>5 \% \cdot \chi_{\text {prob }}^{2}$ is the $\chi^{2}$ fit probability of the $K_{S}^{0}$ daughters.

$\diamond\left|d_{z}^{K_{S}^{0}}\right|<3 \mathrm{~cm} . \quad d_{z}^{K_{S}^{0}}$ is the distance in $z$ between the point of closest approach (POCA) on the collision axis to the $K_{S}^{0}$ trajectory POCA and the interaction point (IP). The resolution in $z$ of the IP measurement is approximately $0.83 \mathrm{~cm}$.

$\diamond d_{x y}^{K_{S}^{0}}<1 \mathrm{~mm} . d_{x y}^{K_{S}^{0}}$ is the radial $(x y)$ distance between the POCA on the collision axis to the $K_{S}^{0}$ trajectory POCA and the IP. 
Chapter 5. Selecting $\boldsymbol{\tau}^{-} \rightarrow \boldsymbol{K}_{S}^{0} \boldsymbol{\pi}^{-} \boldsymbol{\nu}_{\boldsymbol{\tau}}\left(\boldsymbol{K}_{S}^{\mathbf{0}} \rightarrow \boldsymbol{\pi}^{+} \boldsymbol{\pi}^{-}\right)$events

$\diamond L_{x y z}>1.5 \mathrm{~cm} . L_{x y z}$ is the $3 \mathrm{D} K_{S}^{0}$ decay length of the $K_{S}^{0}$ candidate defined to be the distance between the $K_{S}^{0}$ decay vertex and the $K_{S}^{0}$ point of closest approach to the collision axis. It is turned into a signed quantity by taking the dot product of the decay length and $K_{S}^{0}$ LAB 3-momentum unit vectors. (By convention, the decay length vector pointing from the $K_{S}^{0}$ production point to the $K_{S}^{0}$ decay point is positive.) The motivation for making a signed quantity is so that anything negative can immediately be discarded as not being from a $K_{S}^{0}$; it is likely to be background from primary vertices and combinatorics (random-tracks).

$\diamond\left|\cos \Theta_{\text {hel }}^{\pi^{+}}\right|<0.97$. $\cos \Theta_{\text {hel }}^{\pi^{+}}$is the cosine of the angle of the $\pi^{+}$from the decaying $K_{S}^{0}$ in the $K_{S}^{0}$ rest frame with respect to the direction of the $K_{S}^{0}$ in the LAB frame. Bhabha spikes in the data can occur when electrons are misidentified as pions and a pair of oppositely charged electrons are wrongly made into a $K_{S}^{0}$ candidate under the default pion mass hypothesis. These spikes are clearly visible in the $\left|\cos \Theta_{\text {hel }}^{\pi^{+}}\right| \geqslant 0.97$ region. Similar spikes are observed, to a lesser extent, in the $\tau$ Monte Carlo sample due to conversions. For $\left|\cos \Theta_{\text {hel }}^{\pi^{+}}\right| \gtrsim 0.8$ there is an excess of real data over simulated data due to $\Lambda(u d s)$ decays in the data that do not exhibit the same helicity behaviour as their simulated counterparts. In particular, the decay $\Lambda \rightarrow p \pi^{-}$can be mistakenly reconstructed as a $K_{S}^{0}$ by misidentifying a proton as a pion. This decay mode has the largest branching fraction of all $\Lambda$ decays: $\mathcal{B}\left(\Lambda \rightarrow p \pi^{-}\right)=(63.9 \pm 0.5) \%$ [39]. Removing the $\Lambda$ events this way is quite expensive; there is approximately a $20 \%$ net loss of $K_{S}^{0}$ in so doing. Later in this analysis a lepton tag is imposed on the one-prong side of the event which drastically cuts the amount of $u d s$ background and so a cut of $\left|\cos \Theta_{\text {hel }}^{\pi^{+}}\right|<0.8$ is no longer necessary; $\left|\cos \Theta_{\text {hel }}^{\pi^{+}}\right|<0.97$ is chosen to remove Bhabhas (photon conversions).

At the time of production, no radiative Bhabha events were available in SP5. Radiative Bhabha events account to a large extent for the excess in data over the cumulative Monte Carlo samples in the sidebands of the $K_{S}^{0}$ mass window in Figure 5.1. When this was studied with Release-10 data and SP4 Monte Carlo, no radiative Bhabha events survived 

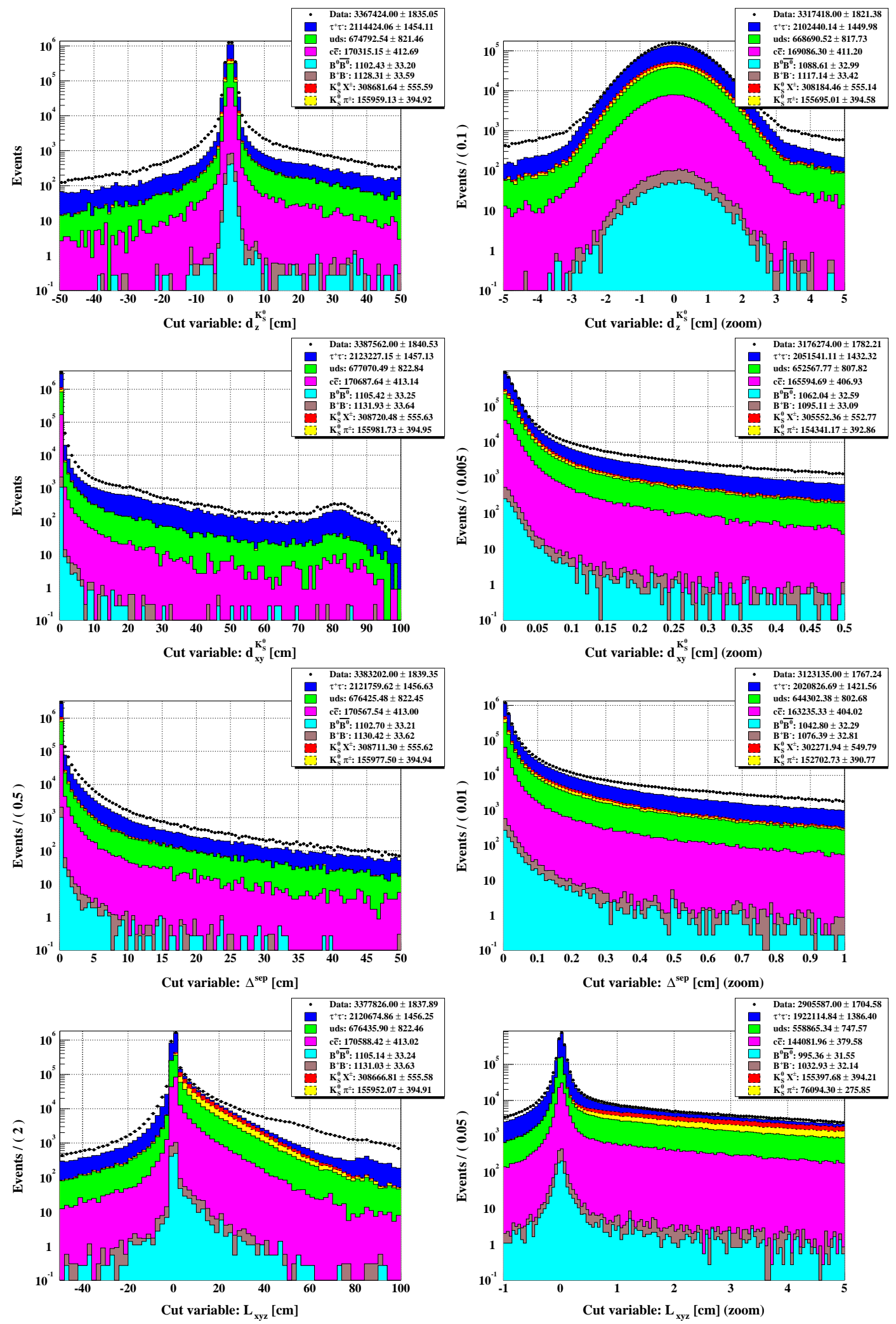

Figure 5.2: $K_{S}^{0}$ selection cut variables. The distributions of the cut variables are shown on a log scale (left) with a zoomed-in view around the cut region (right). 
Chapter 5. Selecting $\boldsymbol{\tau}^{-} \rightarrow \boldsymbol{K}_{S}^{0} \boldsymbol{\pi}^{-} \boldsymbol{\nu}_{\boldsymbol{\tau}}\left(\boldsymbol{K}_{S}^{\mathbf{0}} \rightarrow \boldsymbol{\pi}^{+} \boldsymbol{\pi}^{-}\right)$events
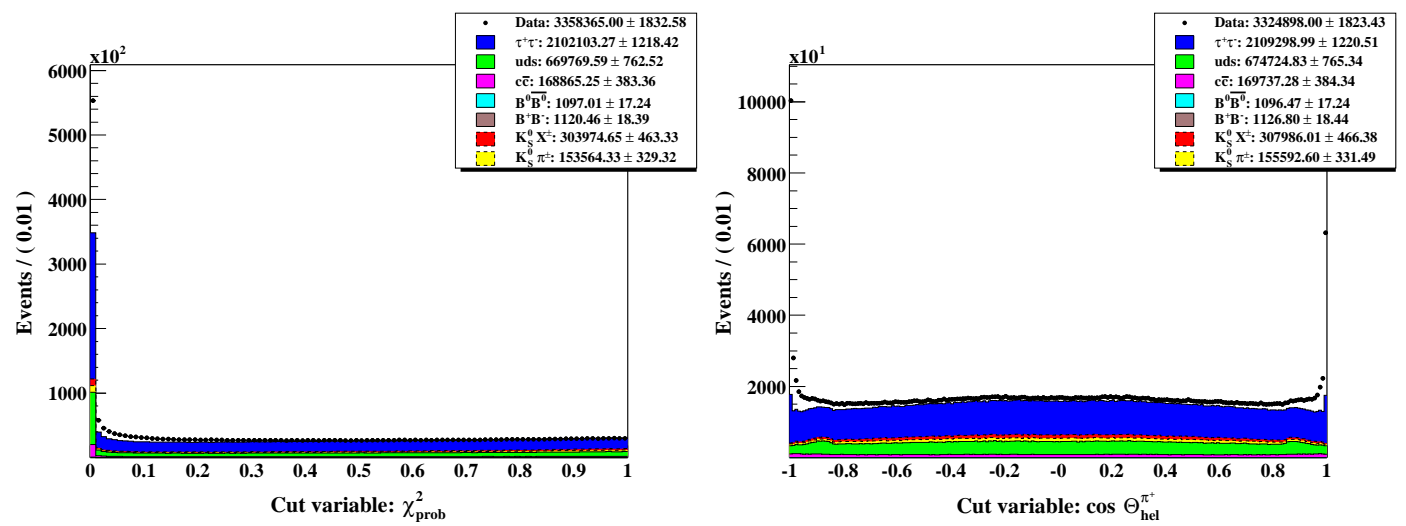

Figure 5.3: $K_{S}^{0}$ selection cut variables. The distributions of the $\chi_{\mathrm{prob}}^{2}$ and $\cos \Theta_{\text {hel }}^{\pi^{+}}$cut variables shown on a linear scale.

this selection. Whilst this is still expected to be true, this should be checked again for the Release-12 data used here once the SP5 radiative Bhabha Monte Carlo becomes available.

\subsubsection{Lepton tagged samples}

Genuine $K_{S}^{0}$ are ubiquitous and exist in a number of decay modes reconstructed at BABAR. More must be done to select $\tau^{ \pm} \rightarrow K_{S}^{0} X^{ \pm} \nu_{\tau}$ events and reduce backgrounds from genuine $K_{S}^{0}$ decays from non- $\tau$ decay modes. A particular source of non- $\tau$ decays including $K_{S}^{0} \mathrm{~s}$ is observed in the $q \bar{q}$ Monte Carlo, i.e. from hadronic decays. In order to reduce $q \bar{q}$ background, tagging on the one-prong (i.e. non-signal) side of the event can be implemented. In this analysis two samples are created using lepton tags. The first sample uses the eMicroTight selector to tag the one-prong side as an electron and the second sample uses the muMicroTight selector to tag the one-prong side as a muon. Since these samples are mutually exclusive, a combined sample is made by simply adding these two together. However, it is important to have information about the two tagged samples individually due to differences in the efficiencies of the selectors which is important, for example, for branching fraction calculations. The selector efficiency differences are discussed in Section 5.6.2. 
Chapter 5. Selecting $\boldsymbol{\tau}^{-} \rightarrow \boldsymbol{K}_{S}^{0} \pi^{-} \boldsymbol{\nu}_{\tau}\left(\boldsymbol{K}_{S}^{0} \rightarrow \boldsymbol{\pi}^{+} \boldsymbol{\pi}^{-}\right)$events

\subsubsection{Identifying the bachelor pion}

The bachelor charged track is associated with the $K_{S}^{0}$ by invoking an algorithm to match a helix to a line resulting in the distance of closest approach of the charged track (helix trajectory) to the $K_{S}^{0}$ (line). The momenta are recomputed at the point of closest approach and the 4-momenta of the $K_{S}^{0}$ and charged track are summed, with a pion mass hypothesis used for the charged track. After this, the piLHVeryTight selector is applied to the bachelor charged track to identify it as a pion. This drastically reduces the $\tau^{-} \rightarrow K^{-} K^{0} \nu_{\tau}$ background.

\subsubsection{Veto $\pi^{0} \mathrm{~s}$}

Two neutral energy cuts are used to reduce the background from events containing unreconstructed $\pi^{0} \mathrm{~s}$. The first is a looser cut on the whole event neutral energy: any event with $\geqslant 1 \mathrm{GeV}$ is rejected. The second cut is tighter; attempting to constrain the neutral energy associated with the signal candidate decay mode. For each neutral candidate whose momentum is within $90^{\circ}$ of the composite $K_{S}^{0} \pi^{ \pm}\left(K^{*}\right)$ candidate momentum, the neutral candidate momentum 4-vector is added together. This quantity is called the " $K^{*}$-side neutral energy", and is required to be less than $250 \mathrm{MeV}$. Even with this cut, there are still around $5 \%$ of selected events that have at least one entry in the piOAllLoose list (i.e. at least one $\pi^{0}$ candidate), which is made from pairs of neutral candidates. Almost all of this $5 \%$ are signal events and so should not be cut away. (A cut of zero $\pi^{0}$ candidates in the piOAllLoose list instead of this neutral cut gives a smaller value of efficiency times purity and so the neutral cut is made in preference: it is a better trade-off between efficiency and purity.)

The distributions of the event neutral energy and $K^{*}$-side neutral energy before selection cuts are shown in Figure 5.4. 

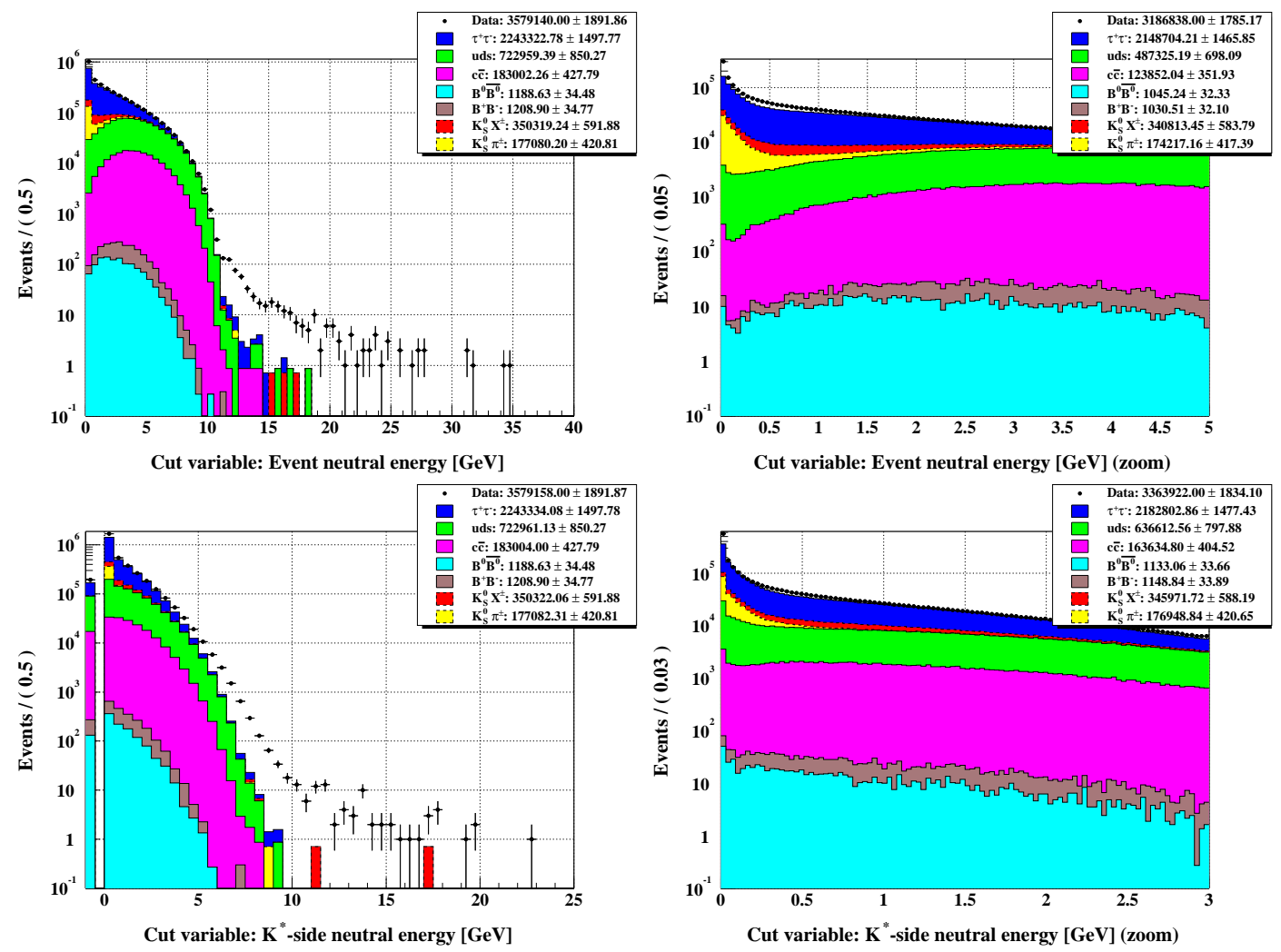

Figure 5.4: Neutral energy cut variables. Neutral energy in the whole event is shown on the top and $K^{*}$-side neutral energy on the bottom. The right hand plots show a zoomed-in view around the cut region of the corresponding plot on its immediate left. (The bin at -1 in the $K^{*}$-side neutral energy plot is filled when the bachelor track is not associated to the $K_{S}^{0}$, i.e. there is no $K^{*}$-side so to speak.) 


\subsection{Signal efficiency}

The signal is defined as $\tau^{-} \rightarrow K_{S}^{0} \pi^{-} \nu_{\tau}\left(K_{S}^{0} \rightarrow \pi^{+} \pi^{-}\right)$. The overall signal efficiency of the selected sample is given by:

$$
\varepsilon_{\mathrm{sig}}=\frac{N_{\mathrm{sig}}^{\mathrm{sel}}}{N_{\mathrm{sig}}^{\mathrm{gen}}},
$$

where $N_{\text {sig }}^{\text {gen }}$ is the number of signal events generated and $N_{\text {sig }}^{\text {sel }}$ is the unweighted number of signal events selected, i.e. without luminosity scaling and data/MC efficiency corrections. The values of $N_{\text {sig }}^{\text {sel }}$ obtained are 23,153,15,285 and 38,438 for the $e$-tag, $\mu$-tag and combined samples respectively. The statistical uncertainty associated with the signal efficiency, as predicted by the Monte Carlo, is given by:

$$
\sigma_{\varepsilon}=\sqrt{\frac{N_{\mathrm{sig}}^{\mathrm{sel}} N_{\mathrm{sig}}^{\overline{\mathrm{sel}}}}{\left(N_{\mathrm{sig}}^{\mathrm{sel}}+N_{\mathrm{sig}}^{\overline{s e l}}\right)^{3}}} .
$$

In Eq. (5.3), $N_{\text {sig }}^{\text {sel }}$ and $N_{\text {sig }}^{\overline{s e l}}$ are the numbers of signal events selected and not selected respectively after the selection procedure has been applied (but no luminosity scaling nor efficiency correction) ${ }^{3}$. The signal efficiencies for the selected samples are given in Table 5.4.

\begin{tabular}{|c||c|}
\hline Sample & $\varepsilon_{\text {sig }}[\%]$ \\
\hline \hline$e$-tag & $2.392 \pm 0.016$ \\
$\mu$-tag & $1.579 \pm 0.013$ \\
Combined & $3.971 \pm 0.020$ \\
\hline
\end{tabular}

Table 5.4: Efficiencies $\left(\varepsilon_{\mathrm{sig}}\right)$ of selecting $\tau^{-} \rightarrow K_{S}^{0} \pi^{-} \nu_{\tau}\left(K_{S}^{0} \rightarrow \pi^{+} \pi^{-}\right)$events in the e-tag, $\mu$-tag and combined (e-tag $+\mu$-tag) samples after selection and PIDweighting.

Table 5.5 shows a breakdown of the number of events surviving each of the selection cuts. In addition to these cuts there is the $\eta_{\mathrm{PID}}<10 \%$ requirement described in Section 5.7.3 that leads to the final efficiency quoted in Table 5.4.

$$
{ }^{3} N_{\mathrm{sig}}^{\overline{\mathrm{sel}}}=N_{\mathrm{sig}}^{\mathrm{gen}}-N_{\mathrm{sig}}^{\mathrm{sel}}
$$




\begin{tabular}{|c|c|c|c|c|c|c|c|c|}
\hline \multirow[t]{2}{*}{ CUT } & \multicolumn{7}{|c|}{ NUMBER OF EVENTS } & \multirow{2}{*}{\begin{tabular}{|c} 
EFFICIENCY [\%] \\
$\tau^{ \pm} \stackrel{K^{* \pm}}{\longrightarrow} K_{S}^{0} \pi^{ \pm} \bar{\nu}_{\tau}$
\end{tabular}} \\
\hline & Data & $\tau^{ \pm} \stackrel{K^{* \pm}}{\longrightarrow} K_{S}^{0} \pi^{ \pm} \bar{\nu}_{\tau}$ & $\tau$ backgrounds & $u \bar{u}, d \bar{d}, s \bar{s}$ & $c \bar{c}$ & $B^{+} B^{-}$ & $B^{0} \bar{B}^{0}$ & \\
\hline AllEvents & & \multirow{2}{*}{\multicolumn{2}{|c|}{$\begin{array}{r}156,776,000 \\
75,576,290\end{array}$}} & $259,974,766$ & $161,670,360$ & $66,293,649$ & $66,148,200$ & \\
\hline TauUser preselection & & & & $15,676,024$ & $5,534,835$ & 54,889 & 66,021 & \\
\hline Additional preselection & $3,579,168$ & 250,745 & $2,925,776$ & 832,792 & 210,279 & 4,005 & 4,385 & 25.91 \\
\hline No. $K_{s}^{0}=1($ KsDefault $)$ & $3,388,092$ & 220,869 & $2,785,860$ & 779,976 & 196,133 & 3,750 & 4,079 & 22.81 \\
\hline Associate $K_{S}^{0}$ to bachelor & $3,200,259$ & 219,916 & $2,678,622$ & 699,797 & 177,441 & 3,297 & 3,597 & 22.72 \\
\hline$K_{S}^{0}$ daughters' fit success & $3,193,905$ & 219,849 & $2,615,375$ & 698,991 & 177,250 & 3,295 & 3,587 & 22.71 \\
\hline$\chi_{\text {prob }}^{2}>5 \%$ & $2,524,992$ & 197,276 & $2,255,233$ & 591,311 & 150,019 & 2,554 & 2,771 & 20.38 \\
\hline$\left|d_{z}^{K_{s}^{0}}\right|<3 \mathrm{~cm}$ & $2,498,427$ & 197,042 & $2,244,828$ & 588,666 & 149,536 & 2,546 & 2,762 & 20.36 \\
\hline$d_{x y}^{K_{S}^{0}}<1 \mathrm{~mm}$ & $2,399,863$ & 193,171 & $2,198,387$ & 574,531 & 145,962 & 2,502 & 2,700 & 19.96 \\
\hline$\Delta^{\text {sep }}<3 \mathrm{~mm}$ & $2,368,948$ & 191,840 & $2,181,755$ & 570,259 & 144,871 & 2,479 & 2,671 & 19.81 \\
\hline$L_{x y z}>1.5 \mathrm{~cm}$ & 382,577 & 154,361 & 163,283 & 154,441 & 40,025 & 331 & 426 & 15.95 \\
\hline$\left|\cos \Theta_{\text {hel }}^{\pi^{+}}\right|<0.97$ & 325,894 & 151,923 & 149,477 & 151,822 & 39,441 & 325 & 415 & 15.70 \\
\hline Event neutral energy $<1 \mathrm{GeV}$ & 135,267 & 106,364 & 67,329 & 17,647 & 1,699 & 14 & 75 & 10.99 \\
\hline$K^{*}$-side neutral energy $<250 \mathrm{MeV}$ & 93,040 & 92,800 & 34,568 & 10,759 & 834 & 9 & 39 & 9.59 \\
\hline$e$-tag or $\mu$-tag & 40,134 & 46,087 & 16,785 & 463 & 238 & 1 & 20 & 4.76 \\
\hline Bachelor PID & 29,923 & 41,116 & 6,343 & 249 & 129 & 1 & 12 & 4.25 \\
\hline
\end{tabular}

Table 5.5: Numbers of events surviving the cuts. The cumulative selection efficiency for the signal mode $\tau^{ \pm} \stackrel{K^{* \pm}}{\longrightarrow} K_{S}^{0} \pi^{ \pm} \bar{\nu}_{\tau}\left(K_{S}^{0} \rightarrow \pi^{+} \pi^{-}\right)$is displayed in the last column. 
Chapter 5. Selecting $\boldsymbol{\tau}^{-} \rightarrow \boldsymbol{K}_{S}^{0} \pi^{-} \boldsymbol{\nu}_{\tau}\left(\boldsymbol{K}_{S}^{0} \rightarrow \boldsymbol{\pi}^{+} \boldsymbol{\pi}^{-}\right)$events

\section{$5.5 \tau$ backgrounds}

Figure 5.5 shows the decay modes of the selected events from the Monte Carlo truth information. The fully inclusive $\tau^{+} \tau^{-}$(blue) number represents all the $\tau$ decay modes selected from everything generated. These modes correspond to those in Table B.1. $K_{S}^{0} X^{ \pm}$(red) represents a semi-inclusive number: all $\tau$ decay modes containing at least one $K_{S}^{0}$ and anything else. As almost all of the fully inclusive $\tau^{+} \tau^{-}$(blue) are covered by the $K_{S}^{0} X^{ \pm}$ (red) events, it shows that most of the remaining backgrounds are from $\tau$ decays including at least one $K_{S}^{0}$. This means that the $K_{S}^{0}$ selection procedure is working extremely well. Finally, the signal $\tau^{ \pm} \stackrel{K^{* \pm}}{\longrightarrow} K_{S}^{0} \pi^{ \pm} \bar{\nu}_{\tau}\left(K_{S}^{0} \rightarrow \pi^{+} \pi^{-}\right)$decay mode (yellow) is shown as part (very nearly all) of mode 7 .

Because some of the background decay modes have large uncertainties associated with them, the individual amounts of each background as a contribution to the total $\tau^{+} \tau^{-}$ number are used to estimate the systematic uncertainty associated with the $\tau$ backgrounds. This is discussed in Section 5.7.6.
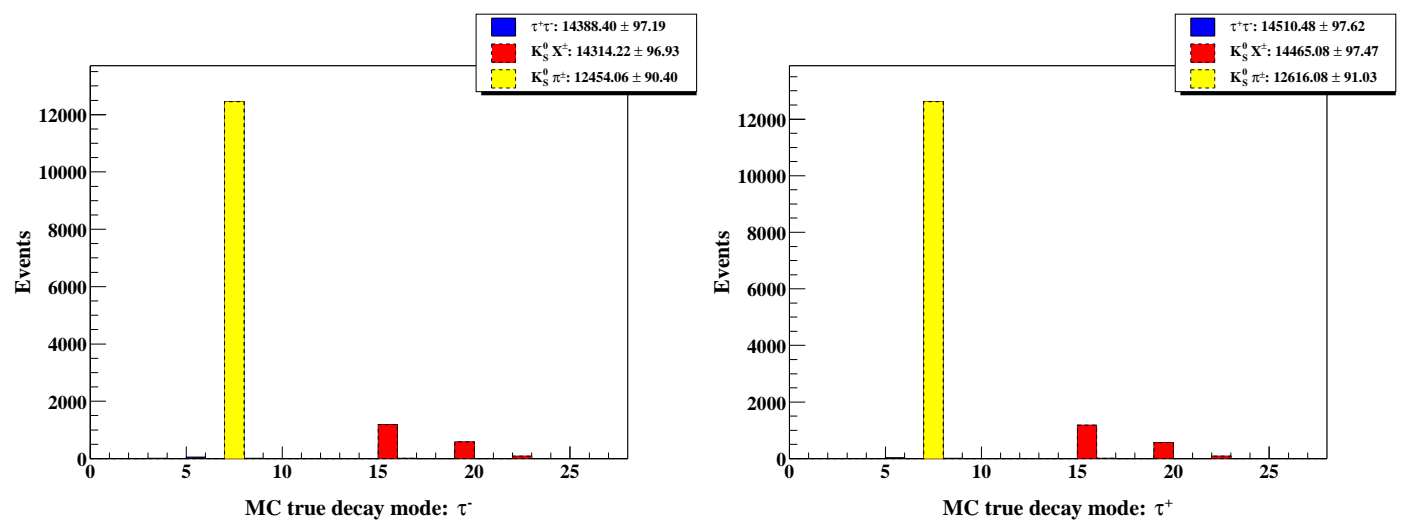

Figure 5.5: $\tau^{-}$(left) and $\tau^{+}$(right) decay mode contributions from the Monte Carlo truth. Each bin represents a particular decay mode (Table B.1). The largest background contributions are from $\tau^{-} \rightarrow K^{0} \pi^{-} \bar{K}^{0} \nu_{\tau}$ (mode 15), $\tau^{-} \rightarrow$ $\pi^{-} \bar{K}^{0} \pi^{0} \nu_{\tau}$ (mode 19) and $\tau^{-} \rightarrow K^{-} K^{0} \nu_{\tau}$ (mode 22). The signal, that comprises part of mode 7 , is also shown (yellow). 
Chapter 5. Selecting $\boldsymbol{\tau}^{-} \rightarrow \boldsymbol{K}_{S}^{0} \pi^{-} \boldsymbol{\nu}_{\tau}\left(\boldsymbol{K}_{S}^{0} \rightarrow \boldsymbol{\pi}^{+} \boldsymbol{\pi}^{-}\right)$events

\subsection{Efficiency corrections}

Since imperfect detector simulation may mean that the reconstruction/selection efficiencies differ between real data and Monte Carlo data, some efficiency corrections are applied to the Monte Carlo data.

\subsection{1 $K_{S}^{0}$ efficiency correction}

For Run $1+2$ in Release-10 and SP4 Monte Carlo, the " $K_{S}^{0}$ Efficiency Task Force" have produced tables binned in $(\mathrm{LAB})$ transverse momentum $\left(p_{T}\right)$, polar angle $(\theta)$ and $L_{x y}$ (actually called $\mathrm{d} r$ in the tables), where $L_{x y}$ is the 2-dimensional ( $x y$ ) decay length of the $K_{S}^{0}$, for various $K_{S}^{0}$ selection criteria and drift chamber voltages [136]. It is possible to read in the efficiencies and uncertainties from the appropriate tables and weight each selected $K_{S}^{0}$ by the corresponding data/MC correction. However, tables have not yet been produced for Release-12 data and SP5 Monte Carlo, so here we use a weightedaverage from the " $K_{S}^{0}$ Efficiency Task Force" for the Release-10 data and SP4 MC. They suggest a global correction (weight) of $0.98 \pm 0.03$ be applied to the Monte Carlo data [136]. This procedure could be refined once the new tables are produced by doing a bin-by-bin weighting of the selected $K_{S}^{0} \mathrm{~s}$ using the tables. So doing should reduce the conservative systematic uncertainty adopted from the current approach. The discussion of the systematic is deferred to Section 5.7.2.

\subsubsection{PID-weighting Monte Carlo}

The selector performance differs between real data and Monte Carlo data and so efficiency corrections to correct the Monte Carlo data to the real data are applied. One approach to making these corrections is to use the PID-killing module [108], which uses efficiency tables ("PID tables") for each selector, that can be run before the user's analysis module. 
Afterwards, the analyst has a pre-PID-killed Monte Carlo data set to work with. An alternative approach is to read in the PID tables directly and devise weights with which the Monte Carlo can be reweighted in order to produce any necessary correction. It is this latter approach that is used here.

The PID selectors used in this analysis are the eMicroTight, muMicroTight and piLHVeryTight selectors, described in Section 4.3.4. PID contributes to the systematic uncertainties associated with the branching fraction measurement $\mathcal{B}\left(\tau^{-} \rightarrow \bar{K}^{0} \pi^{-} \nu_{\tau}\right)$. Lepton selectors are used to tag the non-signal side of the event since a lepton signature is a means of reducing hadronic backgrounds. Hadron selectors are used on the signal side of the event to identify the $\tau^{-} \rightarrow$ (hadrons) $)^{-} \nu_{\tau}$ final state of interest.

The performances of the eMicroTight, muMicroTight and piLHVeryTight selectors are summarised in Table 5.6 and a brief description is in the following sections. A more detailed version can be found in [137] which supports the $\mathcal{B}\left(\tau^{-} \rightarrow(K \pi)^{-} \nu_{\tau}\right)$ document [138]. Since the PID tables have finite binning in $p, \theta$ and $\phi^{4}$, there are implicit cuts applied in the reweighting procedure. For example, if the efficiency correction of a track is sought from an unavailable PID table bin, the event is rejected: no reliable PID information is available for the track. Later, in Section 6.1 (Figure 6.1 and Figure 6.2), there is an illustration of this point.

\subsubsection{1 eMicroTight performance}

A like-for-like comparison of Release-12 real data and SP5 simulated data is presented. Like-for-like means that the same BetaPidCalib selector is used to produce both the real data and simulated data ntuples from which the PID tables are generated. For electrons, the BetaPidCalib radiative Bhabha $(e e \gamma)$ selector is used. The efficiencies and relative efficiencies of the eMicroTight selector to select $e^{-}$and $e^{+}$tracks as a function of LAB momentum $(p)$ are shown, averaged over the whole period 2000-3, in Figure 5.6.

\footnotetext{
${ }^{4}$ In practice the PID tables cover all $\phi$.
} 
Chapter 5. Selecting $\boldsymbol{\tau}^{-} \rightarrow \boldsymbol{K}_{S}^{0} \boldsymbol{\pi}^{-} \boldsymbol{\nu}_{\boldsymbol{\tau}}\left(\boldsymbol{K}_{S}^{\mathbf{0}} \rightarrow \boldsymbol{\pi}^{+} \boldsymbol{\pi}^{-}\right)$events

\begin{tabular}{|c||c|c|c|c|}
\hline Selector & Selected particle & Data compared & Year(s) & $\left\langle\varepsilon_{\text {data }} / \varepsilon_{\mathrm{MC}}\right\rangle[\%]$ \\
\hline \hline eMicroTight & $e^{-}$ & Release-12/SP5 & $2000-3$ & $98.683 \pm 0.009$ \\
\hline eMicroTight & $e^{+}$ & Release-12/SP5 & $2000-3$ & $98.897 \pm 0.012$ \\
\hline muMicroTight & $\mu^{-}$ & Release-12/SP5 & $2000-3$ & $87.765 \pm 0.098$ \\
\hline muMicroTight & $\mu^{+}$ & Release-12/SP5 & $2000-3$ & $87.949 \pm 0.094$ \\
\hline piLHVeryTight & $\pi^{-}$ & Release-10/SP4 & 2001 & $98.577 \pm 0.167$ \\
\hline piLHVeryTight & $\pi^{+}$ & Release-10/SP4 & 2001 & $98.325 \pm 0.162$ \\
\hline
\end{tabular}

Table 5.6: Mean relative efficiencies of real data/simulated data $\left(\left\langle\varepsilon_{\mathrm{data}} / \varepsilon_{\mathrm{MC}}\right\rangle\right)$ for the selectors used in this analysis. The real data are denoted by "Release$x$ " and the Monte Carlo simulated data by "SPx". The mean relative efficiency corresponds to an average efficiency correction ("PID-weight") that is applied to the Monte Carlo in order that the selector performs similarly to how it does for real data. (In practice, this weight is not applied globally but individually for each track.)

\subsubsection{2 muMicroTight performance}

A like-for-like comparison of Release-12 real data and SP5 simulated data is presented. For muons, the BetaPidCalib $\mu \mu \gamma$ selector is used. The efficiencies and relative efficiencies of the muMicroTight selector to select $\mu^{-}$and $\mu^{+}$tracks as a function of LAB momentum $(p)$ are shown, averaged over the whole period 2000-3, in Figure 5.7.

\subsubsection{3 piLHVeryTight performance}

For pions, the BetaPidCalib $D^{*}$ selector is used to make like-for-like comparisons between real data and simulated data. As yet there are no SP5 simulated data tables. In fact, the only simulated table available for pions selected with the BetaPidCalib $D^{*}$ selector uses SP4 Monte Carlo with 2001 conditions. To get the relative efficiencies as a function of $p$, this table is compared with the Release-10 real data tables for 2001 .

The efficiencies and relative efficiencies of the piLHVeryTight selector to select $\pi^{-}$ tracks as a function of $p$ are shown in Figure 5.8. Release-10 real data and SP4 Monte 
Chapter 5. Selecting $\boldsymbol{\tau}^{-} \rightarrow \boldsymbol{K}_{S}^{0} \boldsymbol{\pi}^{-} \boldsymbol{\nu}_{\boldsymbol{\tau}}\left(\boldsymbol{K}_{S}^{\mathbf{0}} \rightarrow \boldsymbol{\pi}^{+} \boldsymbol{\pi}^{-}\right)$events
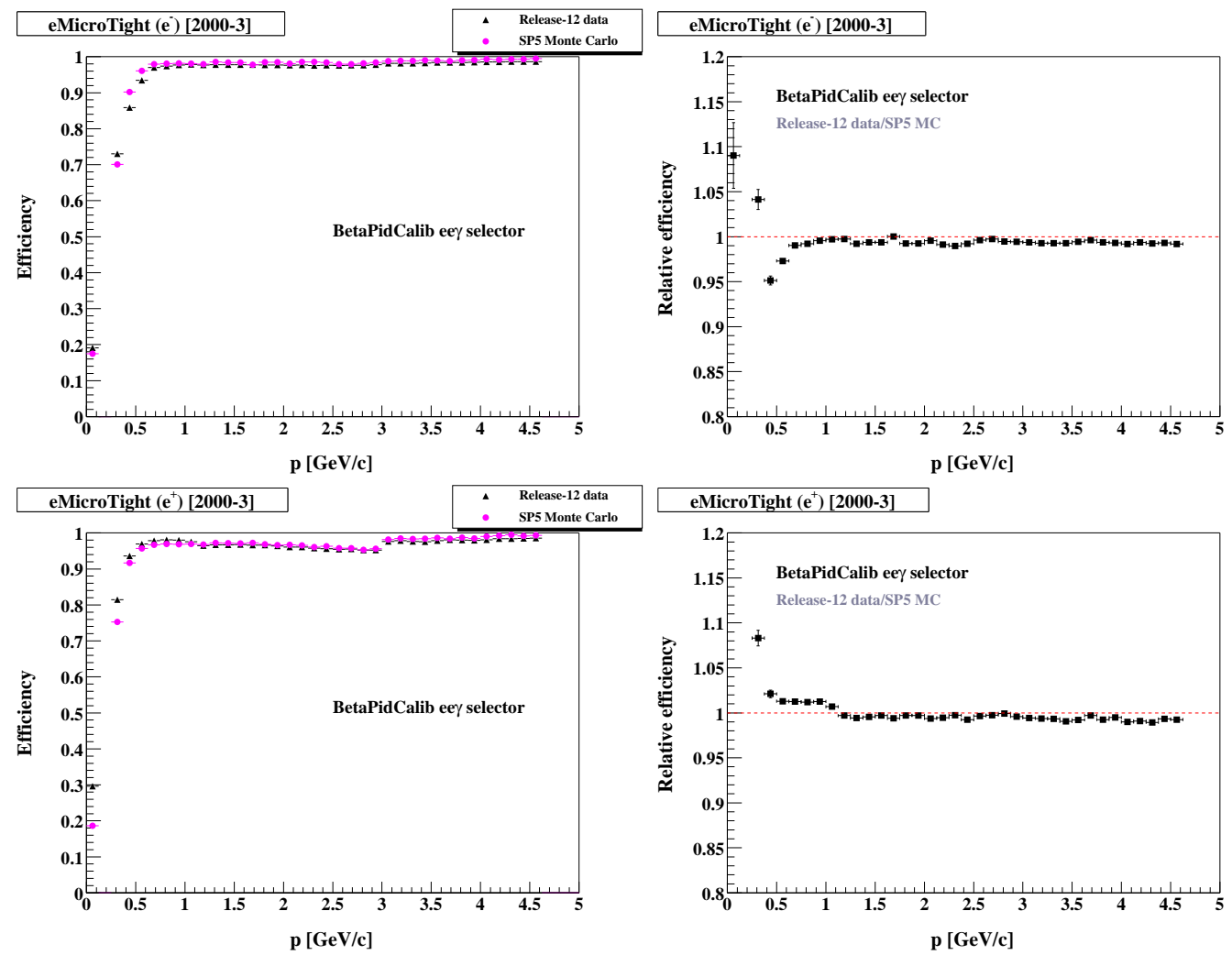

Figure 5.6: Efficiencies (left) and relative efficiencies (right) of $e^{-}$selection (top) and of $e^{+}$selection (bottom) as a function of LAB momentum ( $p$ ) for the eMicroTight selector operating on a sample of Release-12 data and SP5 Monte Carlo selected by the BetaPidCalib ee $\gamma$ selector.

Carlo is primarily compared in the absence of SP5 Monte Carlo tables. However, superimposing the Release-12 real data, it can be seen that the selector performance is stable between the two releases. We expect that the SP5 Monte Carlo tables will show a similar stability of the piLHVeryTight efficiency, or even improvement due to "fine-tuning" of the simulation, between releases.

\subsubsection{Average efficiency correction}

The total data/MC efficiency correction, $\varepsilon_{\text {corr }}$, is made by combining the $K_{S}^{0}$ and PID efficiency corrections. $\varepsilon_{\text {corr }}$ is used to weight the MC and the distribution is shown in 
Chapter 5. Selecting $\boldsymbol{\tau}^{-} \rightarrow \boldsymbol{K}_{S}^{0} \boldsymbol{\pi}^{-} \boldsymbol{\nu}_{\boldsymbol{\tau}}\left(\boldsymbol{K}_{S}^{0} \rightarrow \boldsymbol{\pi}^{+} \boldsymbol{\pi}^{-}\right)$events
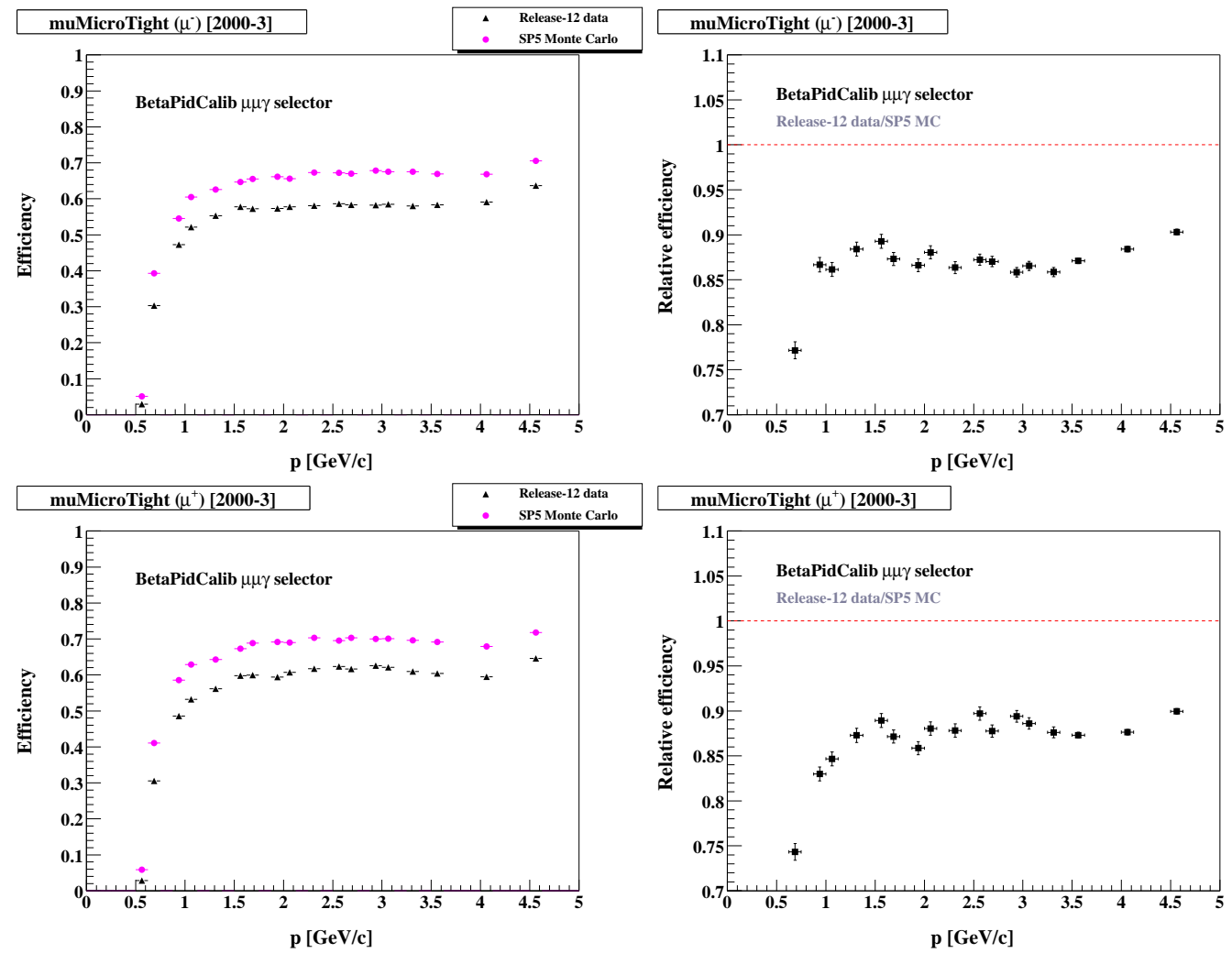

Figure 5.7: Efficiencies (left) and relative efficiencies (right) of $\mu^{-}$selection (top) and of $\mu^{+}$selection (bottom) as a function of LAB momentum ( $p$ ) for the eMicroTight selector operating on a sample of Release-12 data and SP5 Monte Carlo selected by the BetaPidCalib $\mu \mu \gamma$ selector.

Figure 5.9 for the $e$-tag, $\mu$-tag and combined samples. The respective average values obtained are $96.2 \%, 86.6 \%$ and $92.4 \%$ for each of those samples.

\subsection{Systematic uncertainties}

Multiplicative uncertainties affect how the observed signal yields are translated into branching fraction measurements. Table 5.8 lists the multiplicative systematic uncertainties considered in this analysis and the sources of these systematics are described below.

$B A B A R$ has a number of working groups established in order to evaluate common sys- 
Chapter 5. Selecting $\boldsymbol{\tau}^{-} \rightarrow \boldsymbol{K}_{S}^{0} \boldsymbol{\pi}^{-} \boldsymbol{\nu}_{\boldsymbol{\tau}}\left(\boldsymbol{K}_{S}^{\mathbf{0}} \rightarrow \boldsymbol{\pi}^{+} \boldsymbol{\pi}^{-}\right)$events
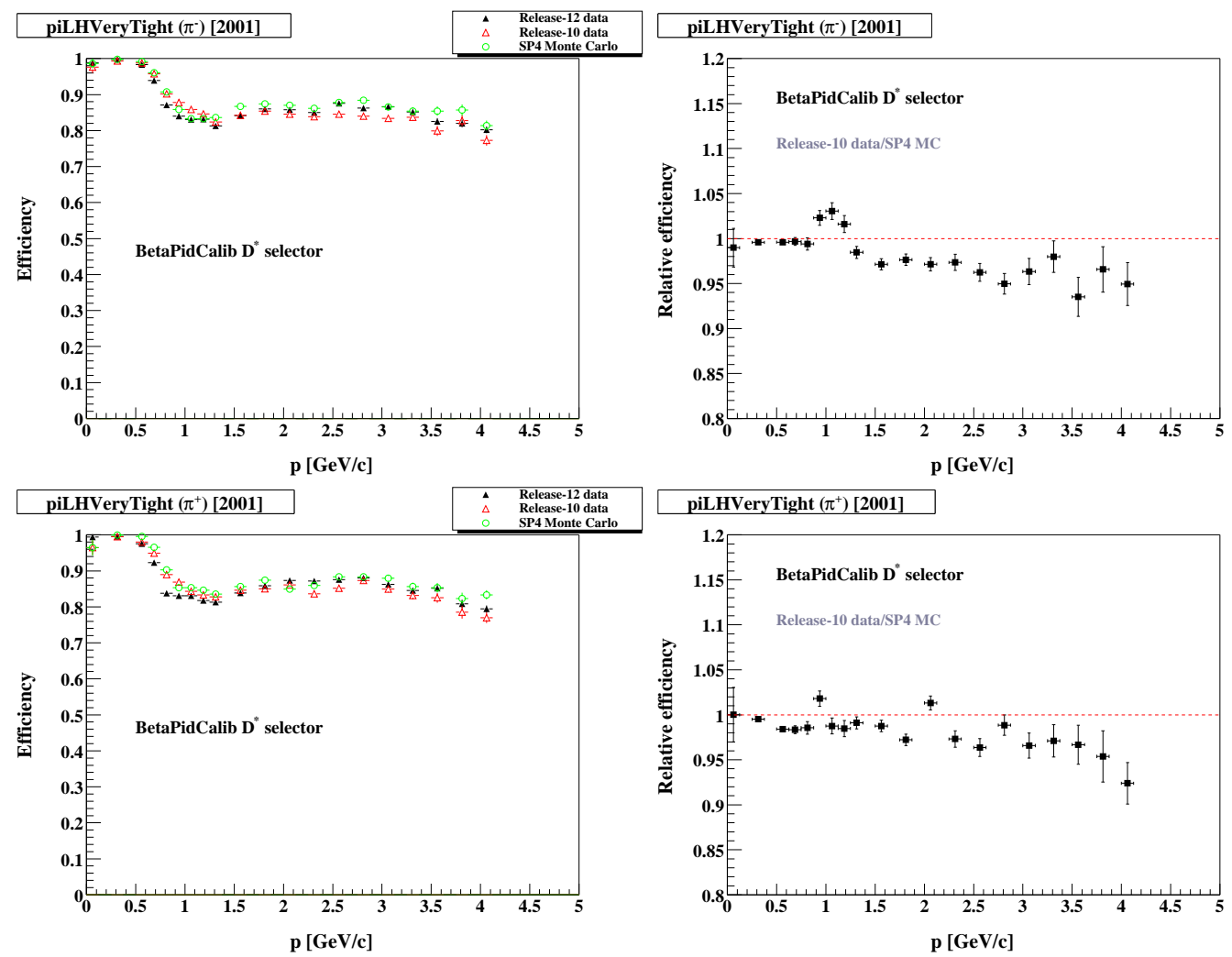

Figure 5.8: Efficiencies (left) and relative efficiencies (right) of $\pi^{-}$selection (top) and of $\pi^{+}$selection (bottom) as a function of LAB momentum ( $p$ ) for the eMicroTight selector operating on a sample of Release-10 data and SP4 Monte Carlo selected by the BetaPidCalib D* selector. The Release-12 data is also overlayed in the efficiency plot.

tematics for analyses. In assessing the various systematic uncertainties we have made use of the results of a number of independent studies by fellow members of the BABAR collaboration. These include the "Tracking Efficiency Task Force" [139] and the " $K_{S}^{0}$ Efficiency Task Force" [136, 140]. Since the 12-series data and SP5 MC has only recently become available, these studies are based on Release-10 data and SP4 MC. However, we expect uncertainties assigned in these documents to give a conservative estimate of the Run 1+2 Release-12 data and SP5 MC values; validation work already indicates that the efficiencies have improved over the corresponding Release-10 data and SP4 MC. For Run 3, some of the uncertainties have increased and we attribute this to different detector conditions. Where appropriate, we estimate the combined Run $1+2+3$ uncertainty as a 
Chapter 5. Selecting $\boldsymbol{\tau}^{-} \rightarrow \boldsymbol{K}_{S}^{0} \boldsymbol{\pi}^{-} \boldsymbol{\nu}_{\boldsymbol{\tau}}\left(\boldsymbol{K}_{S}^{\mathbf{0}} \rightarrow \boldsymbol{\pi}^{+} \boldsymbol{\pi}^{-}\right)$events

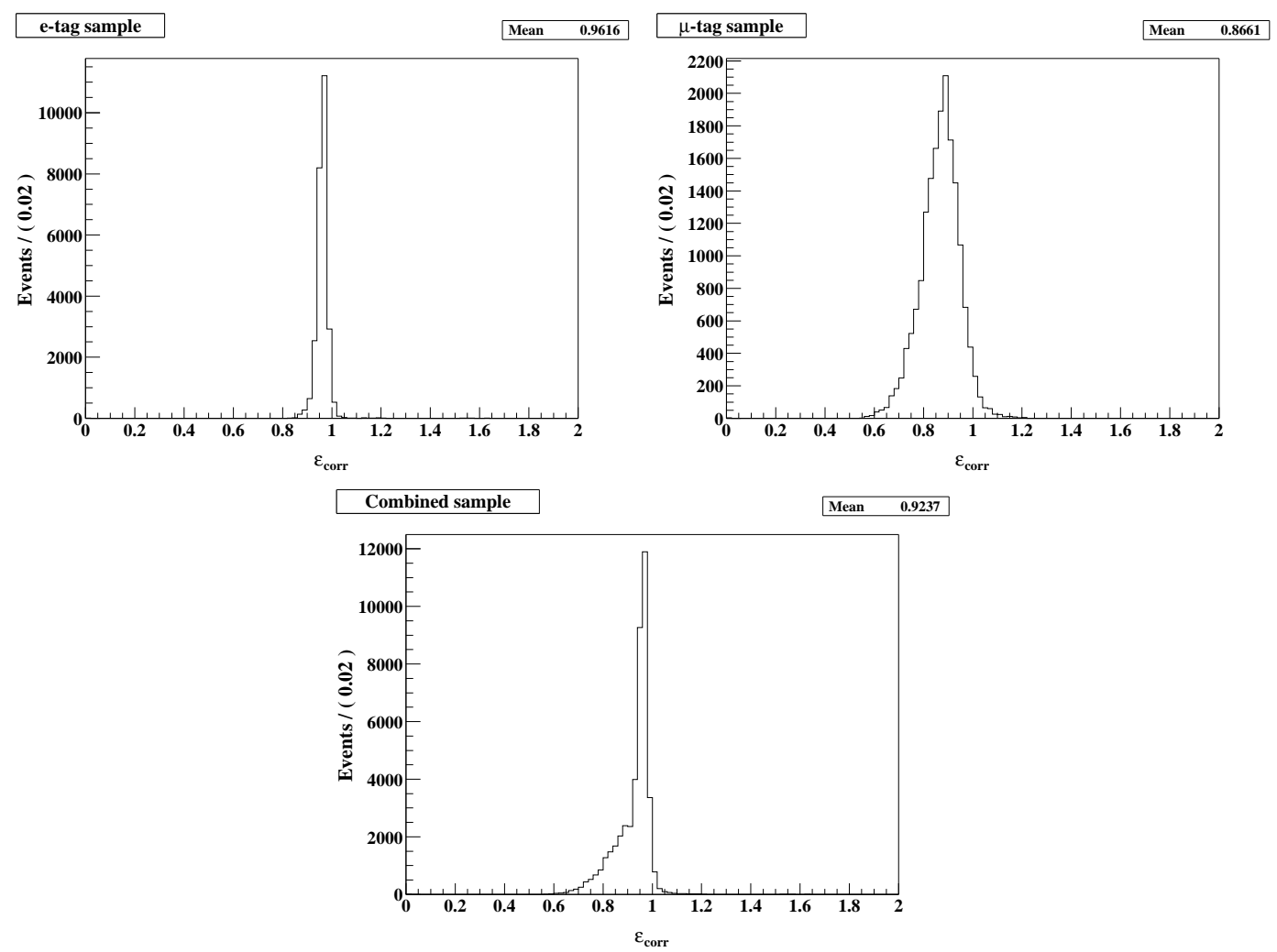

Figure 5.9: Efficiency corrections $\left(\varepsilon_{\text {corr }}\right)$ used to weight the Monte Carlo so that the efficiencies are comparable to the real data.

luminosity weighted mean of the Run $1+2$ and Run 3 uncertainty values.

In this section we make use of a thorough systematic study done for a $B$-physics analysis involving $K_{S}^{0} \mathrm{~s}$ [141]. That analysis shares some common systematics with this analysis and the advantage of using their results (where possible) is that they use some updated numbers for Run 3 Release-12 data and SP5 systematics. Moreover, that analysis is mature: it has been through collaboration-wide review and is in PRL [142]. For some other systematics, we appeal to a recent analysis from the Tau/QED AWG itself which has been through collaboration-wide review and submitted to PRL [143]. There are also some systematics evaluated especially for this analysis. 
Chapter 5. Selecting $\boldsymbol{\tau}^{-} \rightarrow \boldsymbol{K}_{S}^{0} \pi^{-} \boldsymbol{\nu}_{\tau}\left(\boldsymbol{K}_{S}^{0} \rightarrow \boldsymbol{\pi}^{+} \boldsymbol{\pi}^{-}\right)$events

\subsubsection{Tracking efficiency}

The signal efficiency estimate is susceptible to bias caused by physics data and MC simulation discrepancies. For ChargedTracks originating within $1.5 \mathrm{~cm}$ in $x y$ from the beam spot, the relative track reconstruction efficiency for Release-10 data/SP4 MC is $1.000 \pm 0.005$ [139]. In other words, they found that the data/MC agreement is good to $\pm 0.5 \%$ per track for low multiplicity events. We use this number as a conservative estimate of the ratio of tracking efficiency uncertainties between Run 1+2 Release-12 data and SP5 MC. For Run 3, Release-12 data vs SP5 MC comparisons by the "Tracking Efficiency Task Force" indicate the uncertainty may be twice that; the mean relative uncertainty of the data/MC track efficiency ratio for Run $1+2+3$ is therefore estimated to be a luminosity weighted mean of $\pm 0.7 \%$ per track [141]. In the case of $\tau^{-} \rightarrow K_{S}^{0} \pi^{-} \nu_{\tau}$ decays, this corresponds to a correlated uncertainty of $\pm 1.4 \%\left(0.993^{2}=0.986\right)$ on the relative efficiency of the event (excluding the $K_{S}^{0}$ daughter tracks: the $K_{S}^{0}$ efficiency is considered separately and already includes a tracking uncertainty).

\subsection{2 $K_{S}^{0} \rightarrow \pi^{+} \pi^{-}$reconstruction efficiency}

Since the majority of $K_{S}^{0} \mathrm{~s}$ decay beyond $1.5 \mathrm{~cm}$, and the $K_{S}^{0}$ daughters do not necessarily extrapolate to the interaction point, it is expected that the data/MC tracking efficiency ratio uncertainty is different to the $\pm 0.7 \%$ per track. For Release- 10 data and SP4 MC, the uncertainty in the GoodTracksVeryLoose and ChargedTracks relative (data/MC) tracking efficiency for hadron tracks, including daughter pions from $K_{S}^{0} \mathrm{~s}$, is a correlated uncertainty of $\pm 1.3 \%$ per $K_{S}^{0}$ daughter track, leading to $\pm 2.6 \%$ per $K_{S}^{0}$ [136]. The uncertainty in the efficiency of $K_{S}^{0}$ identification is a function of flight distance and momentum, after applying the $K_{S}^{0}$ efficiency corrections. This is obtained from the maximum variation of mean efficiency upon applying alternative parameterizations/cut values for the $K_{S}^{0}$ efficiency computation [140]; for Release-10 Run $1+2$ data and SP4 MC, the " $K_{S}^{0} E$ Efficiency Task Force" evaluate this uncertainty as $\pm 3 \%$ [136]. We use these numbers as a 
conservative estimate of the corresponding Run 1+2 Release-12 data and SP5 MC uncertainty. For Run 3, data vs SP5 comparisons by the "Tracking Efficiency Task Force" indicate the uncertainty may be twice those; the mean relative track efficiency uncertainty for Run $1+2+3$ is therefore estimated to be a luminosity weighted mean of $\pm 1.6 \%$ per $K_{S}^{0}$ daughter track, leading to $\pm 3.8 \%$ per $K_{S}^{0}[141]$.

\subsubsection{PID selectors}

The efficiency correction (data/MC relative efficiency) and corresponding uncertainty is calculated for each track by doing a bin-by-bin comparison with the PID efficiency correction tables for the selectors used. For example, for an e-tag signal event two selectors are used: eMicroTight and piLHVeryTight. On the tag-side of the event the eMicroTight selector is used, so that track's LAB 3-momentum $(\vec{p})$ is passed to a function which returns the relative (data/MC) efficiency and uncertainty in the corresponding eMicroTight correction table bin. Likewise, the bachelor track on the signal side of the event is compared to the corresponding piLHVeryTight data/MC PID table bin. $\eta_{\text {PID }}$, is defined as the total quadrature combination of the relative uncertainties of each track's efficiency correction used to reconstruct the signal event. In the above example that is the quadrature sum of the eMicroTight data/MC efficiency correction relative uncertainty plus the piLHVeryTight data/MC efficiency correction relative uncertainty.

Figure 5.10 shows $\eta_{\mathrm{PID}}$ for the $e$-tag, $\mu$-tag and combined ( $e$-tag $+\mu$-tag) samples. A cut $\left(\eta_{\mathrm{PID}}<10 \%\right)$ was used in the PID-weighting scheme to ensure that this systematic is not biased by outliers and, moreover, that the PID obtained is reliable (well measured). The total PID systematic attributed for each of the $e$-tag, $\mu$-tag and combined samples is $1.34 \%, 5.36 \%$ and $2.94 \%$ respectively. 
Chapter 5. Selecting $\boldsymbol{\tau}^{-} \rightarrow \boldsymbol{K}_{S}^{0} \boldsymbol{\pi}^{-} \boldsymbol{\nu}_{\boldsymbol{\tau}}\left(\boldsymbol{K}_{S}^{\mathbf{0}} \rightarrow \boldsymbol{\pi}^{+} \boldsymbol{\pi}^{-}\right)$events

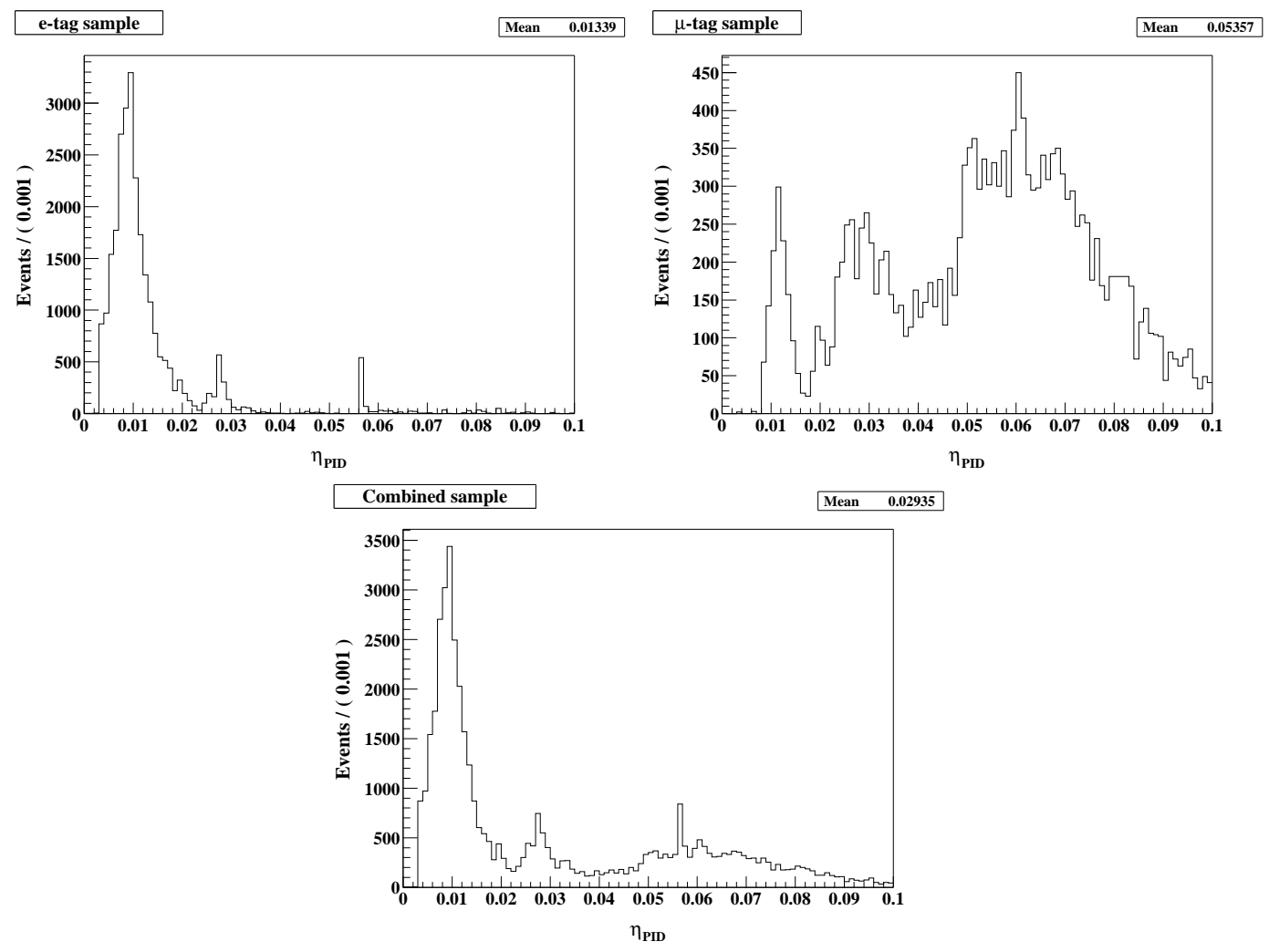

Figure 5.10: $\eta_{\mathrm{PID}}$ for the e-tag, $\mu$-tag and combined samples. The mean value is used as the systematic uncertainty attributed to PID.

\subsubsection{Luminosity and $\tau^{+} \tau^{-}$cross-section}

The relative uncertainty associated with the $\tau^{+} \tau^{-}$pair production cross-section is $2.2 \%$ and is correlated with the luminosity determination relative uncertainty of $1.2 \%$. This correlation arises since the luminosity is determined from $e^{+} e^{-} \rightarrow \mu^{+} \mu^{-}$data which assumes a known production cross-section for this process. This cross-section is calculated using KK2 $\mathrm{f}$, which is the same tool used for determining the $e^{+} e^{-} \rightarrow \tau^{+} \tau^{-}$cross-section, and so the uncertainties in the two cross-sections are highly correlated. Accounting for the correlation, a value of $2.3 \%$ is adopted [143]. 
Chapter 5. Selecting $\boldsymbol{\tau}^{-} \rightarrow \boldsymbol{K}_{S}^{0} \pi^{-} \boldsymbol{\nu}_{\tau}\left(\boldsymbol{K}_{S}^{0} \rightarrow \boldsymbol{\pi}^{+} \boldsymbol{\pi}^{-}\right)$events

\subsubsection{Monte Carlo statistics}

The uncertainty due to Monte Carlo statistics is calculated by dividing Eq. (5.3) by Eq. (5.2). Using the values from Table 5.4 this is computed as $0.67 \%$ for the $e$-tag, $0.82 \%$ for the $\mu$-tag and $0.50 \%$ for the combined ( $e-\operatorname{tag}+\mu$-tag) samples respectively.

\subsection{6 $\tau$ backgrounds}

In the selected sample there are a number of backgrounds from non-signal $\tau$ decay modes. The largest contributions are from $\tau^{-} \rightarrow K^{0} \pi^{-} \bar{K}^{0} \nu_{\tau}$ (mode 15) and $\tau^{-} \rightarrow \pi^{-} \bar{K}^{0} \pi^{0} \nu_{\tau}$ (mode 19), which have branching ratios input into the generator equivalent to the values in Table B.1. Because these modes are not fully reconstructed by the analysis procedure, the non-signal $\tau$ backgrounds tend to be concentrated towards lower mass $\left(\lesssim 0.8 \mathrm{GeV} / c^{2}\right)$. Since these modes have not yet been precisely measured, the branching ratio used as input to the Monte Carlo comes with a significant uncertainty. This uncertainty feeds into the total systematic uncertainty of this analysis. In order to evaluate this systematic, a weighted-sum of the $\tau$ backgrounds is constructed using the Monte Carlo truth for $\tau$ Monte Carlo passing the selection criteria and the uncertainties for each background mode taken from the PDG 2002 [39]. The overall estimated uncertainty due to the $\tau$ backgrounds is given by:

$$
\Delta^{\tau-\mathrm{bkg}}=\sqrt{\sum_{i}\left(w_{i} \frac{\sigma_{i}^{\mathrm{PDG}}}{\mathcal{B}_{i}^{\mathrm{PDG}}}\right)^{2}},
$$

where $w_{i}$ is the weight of background mode $i$, that is the fraction of events of type mode $i$ in the total number of $\tau$ events. $\mathcal{B}_{i}^{\mathrm{PDG}}$ is the branching ratio and $\sigma_{i}^{\mathrm{PDG}}$ is the uncertainty of decay mode $i$ from PDG 2002. Table 5.7 shows the weights and uncertainties of the $\tau$ background modes remaining in the selected sample. The resulting systematic uncertainty ( $\Delta^{\tau-\mathrm{bkg}}$ ) attributed to the $\tau$ background modes is estimated as $1.4 \%$ and is consistent between each of the tagged samples. 
Chapter 5. Selecting $\boldsymbol{\tau}^{-} \rightarrow \boldsymbol{K}_{S}^{0} \boldsymbol{\pi}^{-} \boldsymbol{\nu}_{\boldsymbol{\tau}}\left(\boldsymbol{K}_{S}^{0} \rightarrow \boldsymbol{\pi}^{+} \boldsymbol{\pi}^{-}\right)$events

\begin{tabular}{|c||c|c|c|}
\hline Mode & Decay channel & $w / 10^{-3}$ & $\frac{\sigma^{\text {PDG }}}{\mathcal{B}^{\text {PDG }}}[\%]$ \\
\hline \hline 2 & $\tau^{-} \rightarrow \mu^{-} \bar{\nu}_{\mu} \nu_{\tau}$ & 0.016 & 0.35 \\
3 & $\tau^{-} \rightarrow \pi^{-} \nu_{\tau}$ & 0.263 & 0.99 \\
4 & $\tau^{-} \rightarrow \pi^{-} \pi^{0} \nu_{\tau}$ & 0.064 & 0.55 \\
5 & $\tau^{-} \rightarrow a_{1}^{-} \nu_{\tau}$ & 2.689 & 1.05 \\
6 & $\tau^{-} \rightarrow K^{-} \nu_{\tau}$ & 0.043 & 3.35 \\
7 & $\tau^{-} \rightarrow K^{*-} \nu_{\tau}($ non-sig) & 0.213 & 8.04 \\
8 & $\tau^{-} \rightarrow 2 \pi^{-} \pi^{0} \pi^{+} \nu_{\tau}$ & 0.436 & 2.29 \\
14 & $\tau^{-} \rightarrow K^{-} \pi^{-} K^{+} \nu_{\tau}$ & 0.080 & 11.88 \\
15 & $\tau^{-} \rightarrow K^{0} \pi^{-} \bar{K}^{0} \nu_{\tau}$ & 75.591 & 18.24 \\
16 & $\tau^{-} \rightarrow K^{-} K^{0} \pi^{0} \nu_{\tau}$ & 0.880 & 12.90 \\
18 & $\tau^{-} \rightarrow K^{-} \pi^{-} \pi^{+} \nu_{\tau}$ & 0.041 & 15.15 \\
19 & $\tau^{-} \rightarrow \pi^{-} \bar{K}^{0} \pi^{0} \nu_{\tau}$ & 36.739 & 10.81 \\
22 & $\tau^{-} \rightarrow K^{-} K^{0} \nu_{\tau}$ & 5.324 & 10.39 \\
\hline
\end{tabular}

Table 5.7: $\tau$ background uncertainties from PDG 2002 and their weights in the selected sample. (Note that these numbers correspond to pre-weighted (luminosity and PID) events.)

\subsubsection{Systematics summary}

The total multiplicative systematic uncertainty is the quadrature sum of the individual sources described above and summarised in Table 5.8. The total systematic uncertainties for the $e$-tag, $\mu$-tag and combined (e-tag+ $\mu$-tag) samples are $5.1 \%, 7.3 \%$ and $5.7 \%$ respectively. 
Chapter 5. Selecting $\boldsymbol{\tau}^{-} \rightarrow \boldsymbol{K}_{S}^{0} \pi^{-} \boldsymbol{\nu}_{\tau}\left(\boldsymbol{K}_{S}^{0} \rightarrow \boldsymbol{\pi}^{+} \boldsymbol{\pi}^{-}\right)$events

\begin{tabular}{|c||c|c|c|}
\hline Systematic & $e$-tag & $\mu$-tag & Combined \\
\hline \hline Trk eff. & \pm 1.4 & \pm 1.4 & \pm 1.4 \\
$K_{S}^{0}$ eff. & \pm 3.8 & \pm 3.8 & \pm 3.8 \\
PID & \pm 1.34 & \pm 5.36 & \pm 2.94 \\
$\mathcal{L} / \sigma_{\tau}$ & \pm 2.3 & \pm 2.3 & \pm 2.3 \\
MC statistics & \pm 0.67 & \pm 0.82 & \pm 0.50 \\
$\tau$ backgrounds & \pm 1.4 & \pm 1.4 & \pm 1.4 \\
\hline TOTAL $[\%]$ & \pm 5.1 & \pm 7.3 & \pm 5.7 \\
\hline
\end{tabular}

Table 5.8: The sources of multiplicative systematic uncertainty considered for $\tau^{-} \rightarrow K_{S}^{0} \pi^{-} \nu_{\tau}$ decays. 


\section{Studies of $\tau^{-} \rightarrow K_{S}^{0} \pi^{-} \nu_{\tau}$ decay}

\subsection{Reconstructed and identified tracks}

\subsubsection{Lepton (one-prong) tagged track}

The Monte Carlo has been scaled to the data using the appropriate luminosities and the efficiency corrections described in Section 5.6 have been applied. To ensure that the efficiency corrections are behaving sensibly, Figure 6.1 shows the $p, \theta$ and $\phi$ distributions for the electron track selected with the eMicroTight selector and the muon track with the muMicroTight selector. Within the systematic uncertainties summarised in Table 5.8, the real and simulated data agree well.

\subsubsection{Pion (bachelor) identified track}

Figure 6.2 shows the $p, \theta$ and $\phi$ distributions for the bachelor track identified as a pion with the piLHVeryTight selector. Again, within the systematic uncertainties summarised 

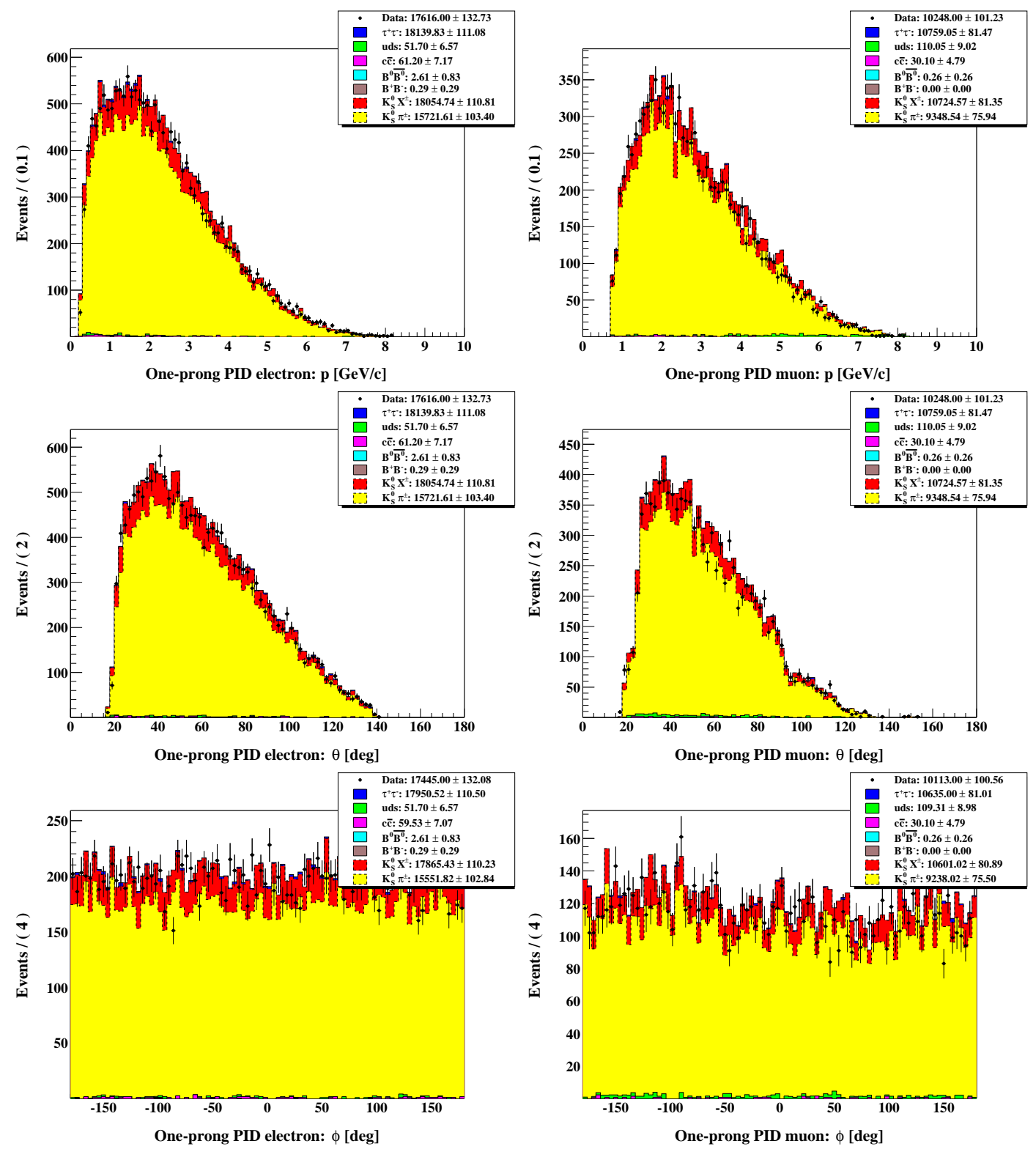

Figure 6.1: One-prong (tag-side) particle kinematic distributions for electron tagged events using the eMicroTight selector (left) muon tagged events using the muMicroTight selector (right). 
in Table 5.8, the real and simulated data agree well.
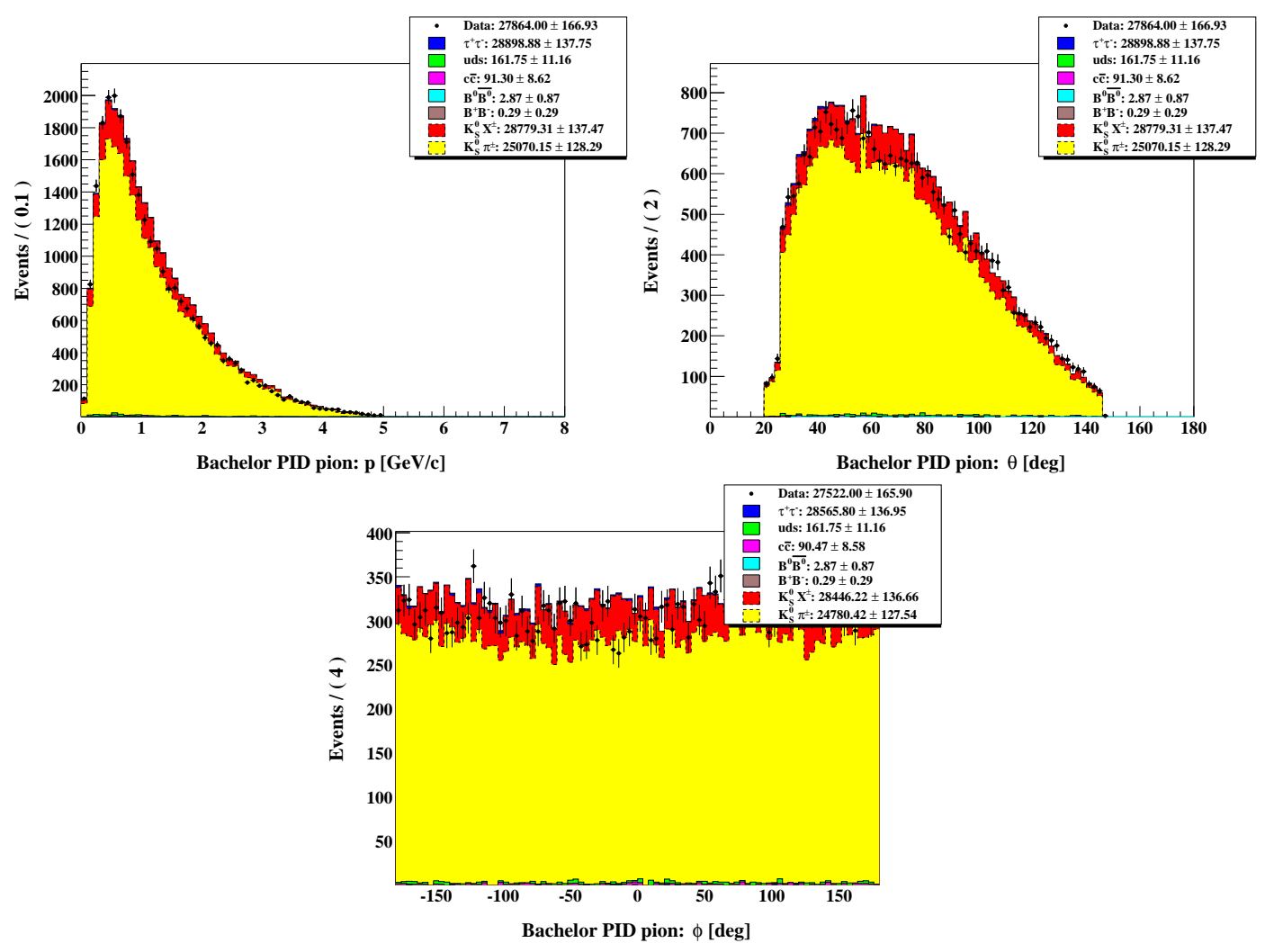

Figure 6.2: Bachelor particle kinematic distributions for the signal-side track associated with the $K_{S}^{0}$ and identified as a pion with the piLHVeryTight selector. The sharp cuts in the $\theta$ distribution correspond to the ends of the PID table bins at $20.05^{\circ}$ and $146.10^{\circ}$.

\subsection{Selected $K_{S}^{0}$ properties}

\subsection{1 $K_{S}^{0} \operatorname{mass}$}

Figure 6.3 shows the $K_{S}^{0}$ mass distribution of the selected events. The purity of the $K_{S}^{0}$ selection from $\tau$ decays is $99.6 \%$. An unbinned extended maximum likelihood fit to the $K_{S}^{0}$ mass in data is performed using a double Gaussian probability density function (PDF) for the $K_{S}^{0}$ signal, allowing the means and widths of the Gaussians to float, and a Chebychev 
PDF for any accidental background (from Figure 6.3, any accidental background is all but non-existent). Both of these functions are defined in the RooF it $[131,132]$ package described in Section 4.8.2. A Chebychev function is used for the non- $K_{S}^{0}$ background because it has better orthogonality and convergence properties than "ordinary" polynomials ${ }^{1}$ [126]. Figure 6.4 shows the result of the fit to the real data. The fitted mass is higher than the PDG value, $m_{K_{S}^{0}}^{\mathrm{PDG}}=497.672 \pm 0.031 \mathrm{MeV} / c^{2}$ [39], by approximately $4 \sigma$. This seems to be a consistent feature of BABAR analyses of the $K_{S}^{0}$. The mass of the $K_{S}^{0}$ from the Monte Carlo data is slightly higher still. Neither of these discrepancies are fully understood, but they do not affect this analysis.

\subsection{2 $K_{S}^{0}$ momentum and angular distributions}

Figure 6.5 shows the (LAB) transverse momentum $\left(p_{T}\right)$, polar angle $(\theta)$ and $2 \mathrm{D}$ flight length $\left(L_{x y}\right)$ of the selected $K_{S}^{0}$. The $K_{S}^{0}$ efficiency correction tables are binned in these quantities, as described in Section 5.6.1. The real and simulated data agree well even though a global correction, rather than a bin-by-bin weighting, has been used.

Since the $K_{S}^{0}$ is a pseudoscalar $\left(J^{P}=0^{-}\right)$it should have no angular dependence exhibited in a distribution of the helicity cosine, $\cos \Theta_{\text {hel }}^{\pi^{+}}$. Because of detector acceptance this distribution is not flat, as shown in Figure 6.6. Since $\cos \Theta_{\text {hel }}^{\pi^{+}}$should (theoretically) be flat, it was hoped to use the $\cos \Theta_{\text {hel }}^{\pi^{+}}$plot as a diagnostic tool during selection to check that the selected $K_{S}^{0}$ were not being angularly biased by the selection procedure. This is important so that the simulation does not have to work so hard to get a good match with real data, which could lead to problems later in the $K \pi s$-wave search. Instead, because in practice $\cos \Theta_{\text {hel }}^{\pi^{+}}$is not flat, the dihedral angle $\left(\phi_{\mathrm{D}}\right)$ is used as a diagnostic tool. The dihedral angle is defined as the angle between the normal to the plane identified by the bachelor $\pi$ and $K_{S}^{0}$ momenta and the normal to the plane identified by the $K_{S}^{0}$ decay products' momenta. It is expected that this $\phi_{\mathrm{D}}$ distribution should be flat. Figure 6.6 also

\footnotetext{
${ }^{1}$ A linear polynomial could be chosen for the background PDF. Since in this case the backgrounds are so small this distinction is insignifi cant.
} 

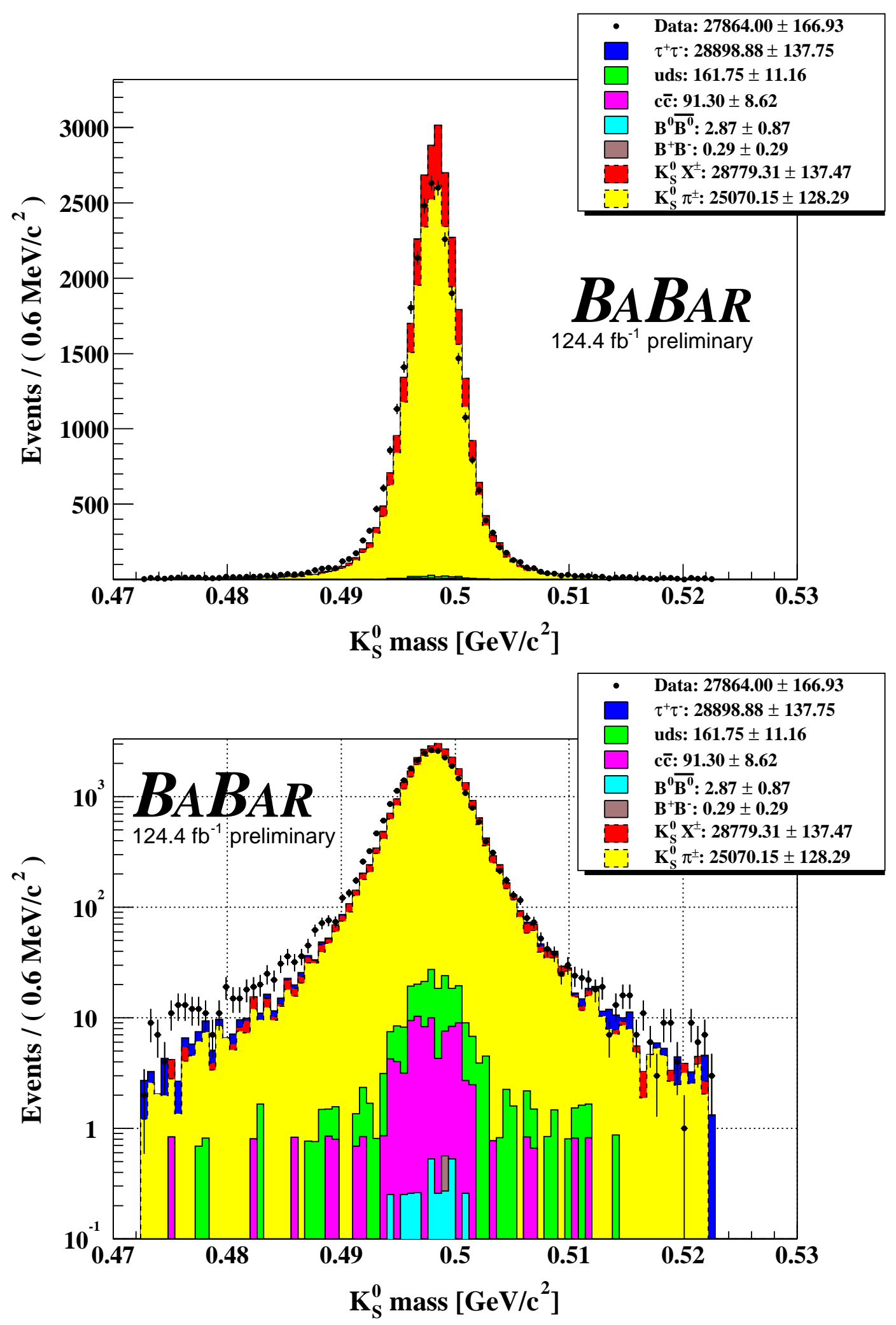

Figure 6.3: Reconstructed $K_{S}^{0}$ mass after selection and efficiency corrections. 


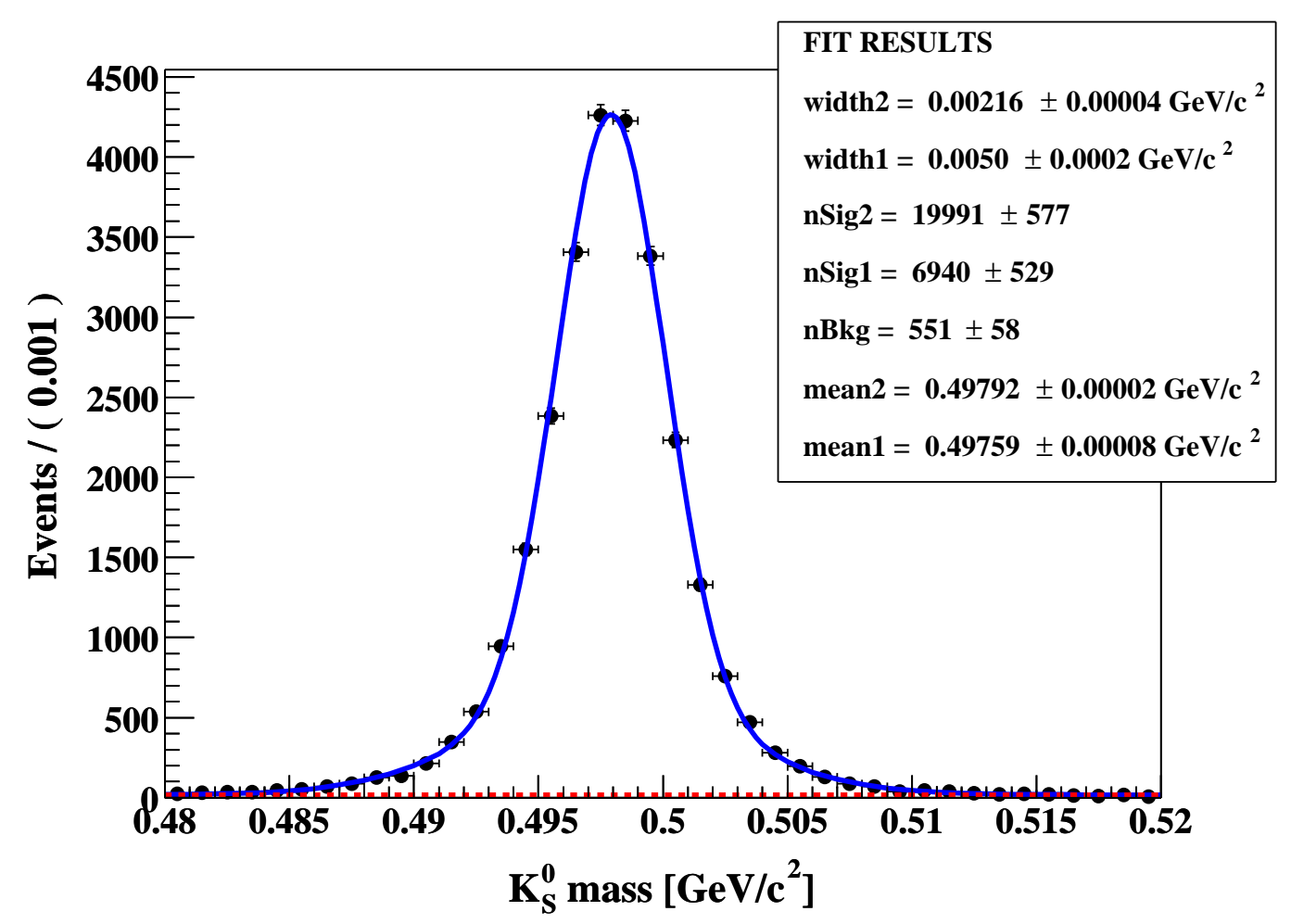

Figure 6.4: Fit to the reconstructed $K_{S}^{0}$ mass in real data. Double Gaussian signal PDF and Chebychev background PDF. The Chebychev component is shown as the red dashed line. 

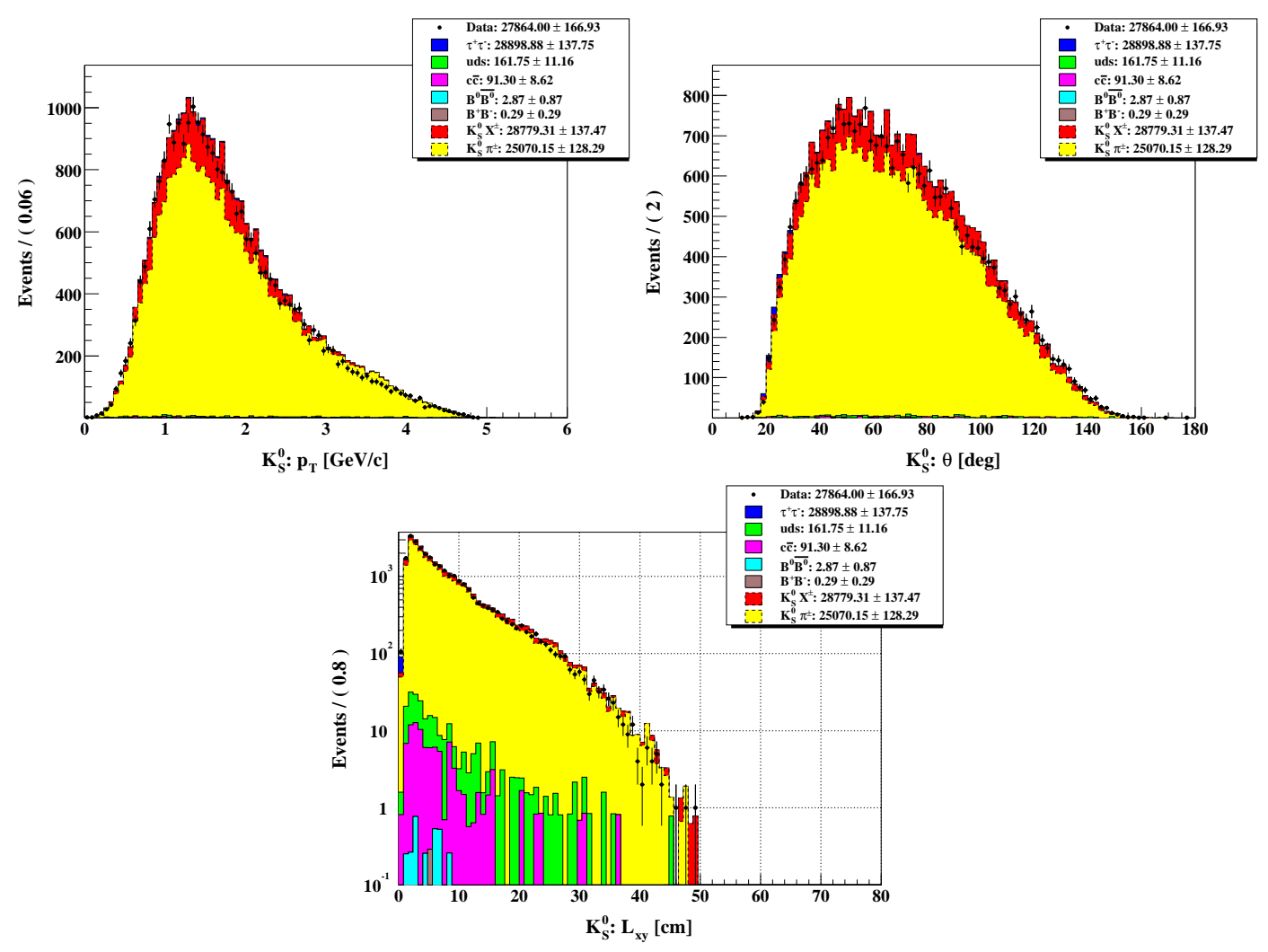

Figure 6.5: $p_{T}, \theta$ and $2 D$ decay length $\left(L_{x y}\right)$ of selected $K_{S}^{0}$ particles. The quantities are in the LAB system. 
shows the distribution of $\phi_{\mathrm{D}}$ for the selected $K_{S}^{0}$ particles, which is flat within the expected uncertainties.
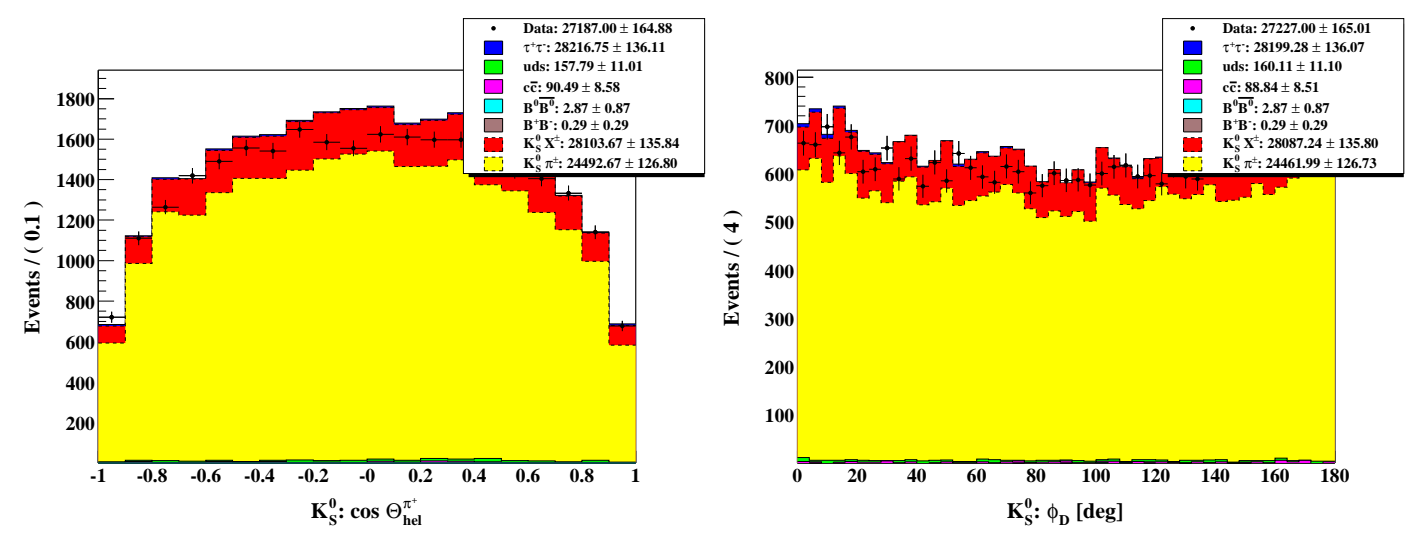

Figure 6.6: $K_{S}^{0}$ helicity cosine, $\cos \Theta_{\text {hel }}^{\pi^{+}}$, (left) and dihedral angle, $\phi_{\mathrm{D}}$, (right).

\subsection{3 $K_{S}^{0}$ decay distributions}

Figure 6.7 shows the 3D decay length $\left(L_{x y z}\right)$ and proper decay time (in PDG $K_{S}^{0}$ mean lifetime units) distributions for the selected $K_{S}^{0}$ candidates. There is a very good match between real data and Monte Carlo data over the range of the plots. Since proper decay time is in $K_{S}^{0}$ lifetime units, its slope should (on average) be -1. It is. However, just using 1-3 events ${ }^{2}$ as defined by TauUser (i.e. based on GoodTracksVeryLoose) can significantly alter the slope: the $K_{S}^{0}$ selection is therefore biased and so should be avoided.

\footnotetext{
${ }^{2}$ Just using 1-3 events, for instance, preferentially selects $K_{S}^{0}$ candidates with a shorter decay length (and therefore shorter proper decay time) because of the DOCA requirements demanded on all three tracks (GoodTracksVeryLoose) on the signal side. Particularly interesting was using 1-1 and 1-3 events, i.e. missing out 1-2 events, which puts a dip at around $1.5-2.0 \mathrm{~cm}$ in the plot of proper decay time.
} 

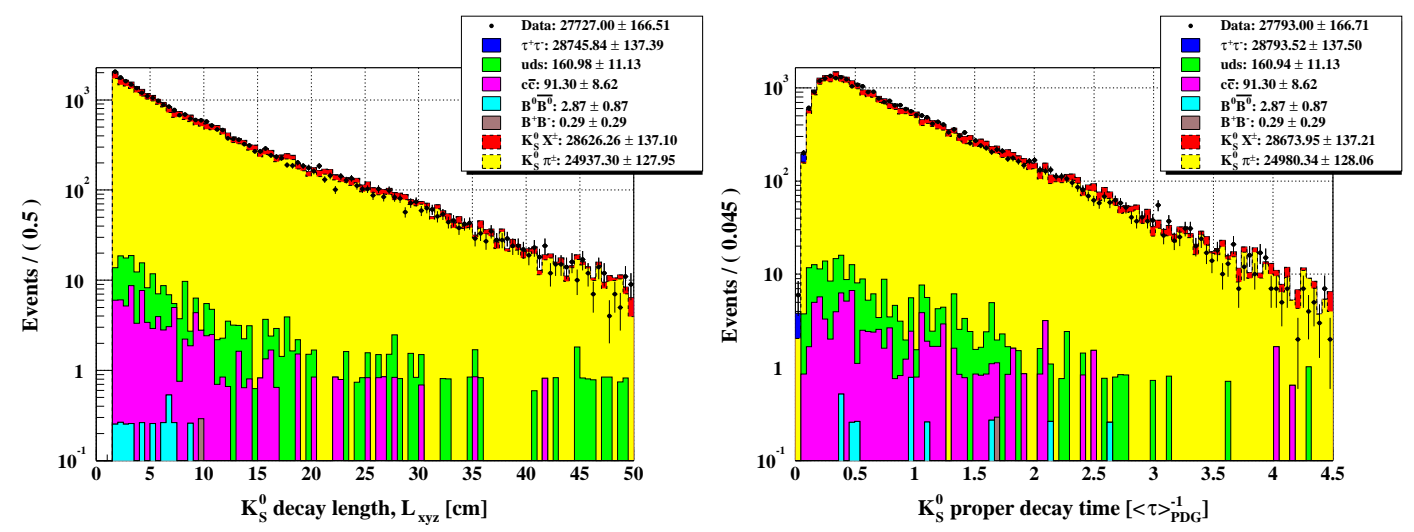

Figure 6.7: $K_{S}^{0} 3 D$ decay length, $L_{x y z}$, (left) and proper decay time in $K_{S}^{0}$ mean lifetime units (right) for the selected $K_{S}^{0} \mathrm{~s}$. On average, the slope of the proper decay time distribution is -1 .

\section{3 $\mathcal{B}\left(\tau^{-} \rightarrow \bar{K}^{0} \pi^{-} \nu_{\tau}\right)$}

$\mathcal{B}\left(\tau^{-} \rightarrow \bar{K}^{0} \pi^{-} \nu_{\tau}\right)$ can be computed by replacing ${ }^{3}$ the $\tau^{-} \stackrel{K^{*-}}{\longrightarrow} K_{S}^{0} \pi^{-} \nu_{\tau}\left(K_{S}^{0} \rightarrow \pi^{+} \pi^{-}\right)$ signal efficiency, $\varepsilon_{\mathrm{sig}}$, with the $\tau^{-} \rightarrow \bar{K}^{0} \pi^{-} \nu_{\tau}$ signal efficiency, $\varepsilon_{\mathrm{sig}}^{\prime}$. The redefined signal efficiency is therefore given by:

$$
\varepsilon_{\text {sig }}^{\prime}=\varepsilon_{\text {sig }} \times \mathcal{B}\left(K^{0}=K_{S}^{0}\right) \times \mathcal{B}\left(K_{S}^{0} \rightarrow \pi^{+} \pi^{-}\right),
$$

where the values of $\varepsilon_{\text {sig }}$ are taken from Table 5.4 and the relative uncertainty associated with $\varepsilon_{\text {sig }}^{\prime}$ is identical to that associated with $\varepsilon_{\text {sig }}$. The motivation for doing this at this stage is that measurements of $\mathcal{B}\left(\tau^{-} \rightarrow \bar{K}^{0} \pi^{-} \nu_{\tau}\right)$ appear explicitly in the PDG review [39] and so quoting this number allows an easier comparison with other experiments to be made. The resulting efficiency $\left(\varepsilon_{\text {sig }}^{\prime}\right)$ values are $0.820 \%, 0.542 \%$ and $1.362 \%$ for the $e$-tag, $\mu$-tag and combined samples respectively.

The branching ratio $\mathcal{B}\left(\tau^{-} \rightarrow \bar{K}^{0} \pi^{-} \nu_{\tau}\right)$ is given by:

$$
\mathcal{B}\left(\tau^{-} \rightarrow \bar{K}^{0} \pi^{-} \nu_{\tau}\right)=\frac{1}{2 N_{\tau \tau}} \frac{N_{\text {data }}-N_{\text {bkg }}}{\varepsilon_{\text {sig }}^{\prime}},
$$

\footnotetext{
${ }^{3}$ This effectively reverses what was done in Eq. (5.1) which was adopted since $\tau^{-} \rightarrow \bar{K}^{0} \pi^{-} \nu_{\tau}\left(K_{S}^{0} \rightarrow\right.$ $\pi^{+} \pi^{-}$) decays are actually reconstructed in this analysis.
} 
where $N_{\tau \tau}$ is the number of $\tau^{+} \tau^{-}$pairs expected in the data, $N_{\text {data }}$ is the number of selected events in real data, $N_{\mathrm{bkg}}$ is the number of selected events in the background Monte Carlo. The number of $\tau^{+} \tau^{-}$pairs expected in the data is

$$
N_{\tau \tau}=\sigma_{\tau} \mathcal{L}_{\text {data }}=110,683,856
$$

and is subject to correlated uncertainties in the cross-section and luminosity as discussed in Section 5.7.4. Table 6.1 shows the numbers of real data and Monte Carlo data that are used in Eq. (6.2) to calculate $\mathcal{B}\left(\tau^{-} \rightarrow \bar{K}^{0} \pi^{-} \nu_{\tau}\right)$. The signal number corresponds to the decay $\tau^{ \pm} \stackrel{K^{* \pm}}{\longrightarrow} K_{S}^{0} \pi^{ \pm} \bar{\nu}_{\tau}$ followed by $K_{S}^{0} \rightarrow \pi^{+} \pi^{-}$and is a component of the inclusive $\tau^{+} \tau^{-}$number.

\begin{tabular}{|c||c|c|c|}
\hline Data & $e$-tag & $\mu$-tag & Combined \\
\hline \hline Real & $17616.00 \pm 132.73$ & $10248.00 \pm 101.23$ & $27864.00 \pm 166.93$ \\
\hline$\tau^{+} \tau^{-}$ & $18139.83 \pm 111.08$ & $10759.05 \pm 81.47$ & $28898.88 \pm 137.75$ \\
$u d s$ & $51.70 \pm 6.57$ & $110.05 \pm 9.02$ & $161.75 \pm 11.16$ \\
$c \bar{c}$ & $61.20 \pm 7.17$ & $30.10 \pm 4.79$ & $91.30 \pm 8.62$ \\
$B^{0} \bar{B}^{0}$ & $2.61 \pm 0.83$ & $0.26 \pm 0.26$ & $2.87 \pm 0.87$ \\
$B^{+} B^{-}$ & $0.29 \pm 0.29$ & - & $0.29 \pm 0.29$ \\
\hline Signal & $15721.61 \pm 103.40$ & $9348.54 \pm 75.94$ & $25070.15 \pm 128.29$ \\
\hline
\end{tabular}

Table 6.1: Numbers of real and Monte Carlo data events remaining in the selected samples. The signal number is $\tau^{ \pm} \stackrel{K^{* \pm}}{\longrightarrow} K_{S}^{0} \pi^{ \pm} \bar{\nu}_{\tau}$ followed by $K_{S}^{0} \rightarrow \pi^{+} \pi^{-}$ and is a component of the inclusive $\tau^{+} \tau^{-}$number.

The resulting branching ratios obtained are shown in Table 6.2 for the $e$-tag, $\mu$-tag and combined samples. The systematic uncertainties quoted correspond to the total reached in Table 5.8. Since the systematic uncertainty associated with muon selection is so large, the benefit in statistics achieved by combining the $e$-tag and $\mu$-tag samples is out-weighed by the worsening of the systematic uncertainty. Therefore, the best measurement of $\mathcal{B}\left(\tau^{-} \rightarrow\right.$ $\left.\bar{K}^{0} \pi^{-} \nu_{\tau}\right)$ made here comes solely from the $e$-tag sample. Figure 6.8 shows how our best measurement of $\mathcal{B}\left(\tau^{-} \rightarrow \bar{K}^{0} \pi^{-} \nu_{\tau}\right)$ compares with the current world average from PDG 2002 [39]. Our preliminary result is the world's most precise measurement of $\mathcal{B}\left(\tau^{-} \rightarrow\right.$ $\left.\bar{K}^{0} \pi^{-} \nu_{\tau}\right)$ to date. 


\begin{tabular}{|c||c|}
\hline Sample & $\mathcal{B}\left(\tau^{-} \rightarrow \bar{K}^{0} \pi^{-} \nu_{\tau}\right)[\%]$ \\
\hline \hline$e$-tag & $0.830 \pm 0.005$ (stat) \pm 0.042 (syst) \\
$\mu$-tag & $0.725 \pm 0.007$ (stat) \pm 0.053 (syst) \\
Combined & $0.789 \pm 0.005$ (stat) \pm 0.045 (syst) \\
\hline
\end{tabular}

Table 6.2: $\mathcal{B}\left(\tau^{-} \rightarrow \bar{K}^{0} \pi^{-} \nu_{\tau}\right)$ measured in this analysis. The highlighted row gives our most precise measurement.

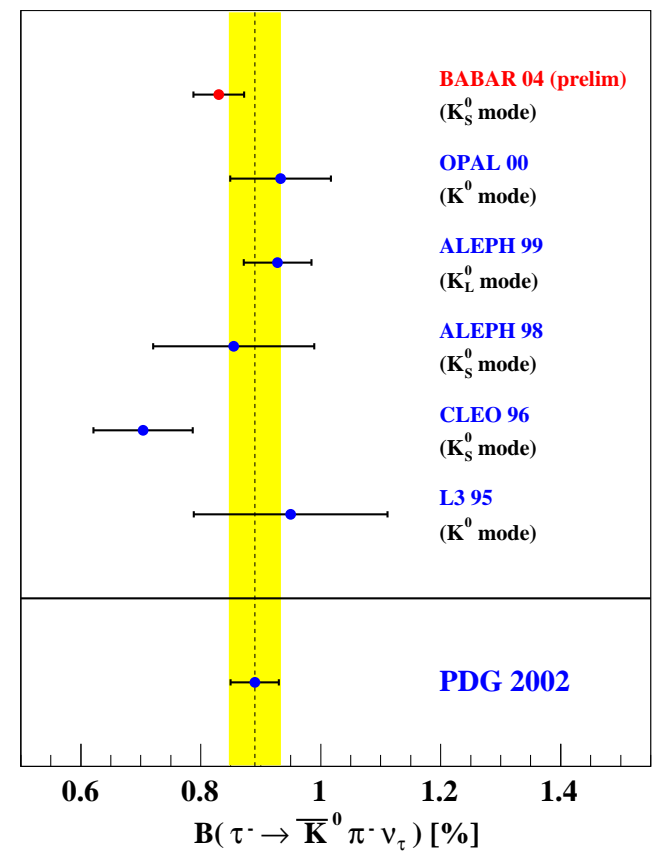

Figure 6.8: World measurements of $\mathcal{B}\left(\tau^{-} \rightarrow \bar{K}^{0} \pi^{-} \nu_{\tau}\right)$. This analysis provides the world's most precise measurement to date: $\mathcal{B}\left(\tau^{-} \rightarrow \bar{K}^{0} \pi^{-} \nu_{\tau}\right)=$ $(0.830 \pm 0.005$ (stat) \pm 0.042 (syst) $) \%$.

\section{4 $\tau^{-} \rightarrow K_{S}^{0} \pi^{-} \nu_{\tau}$ invariant mass spectra}

\subsection{1 $K \pi$ mass distribution}

Figure 6.9 shows the $K \pi$ mass spectrum from $\tau^{-} \rightarrow K_{S}^{0} \pi^{-} \nu_{\tau}$ decays. The real and simulated data agree well for almost all $K \pi$ mass, particularly in the $K^{*}(892)$ peak region. 
At lower mass $\left(\lesssim 0.8 \mathrm{GeV} / c^{2}\right)$, there is some discrepancy between the real and simulated data. This is, however, not too surprising since this region is where the $\tau$ backgrounds are highest and these come with large uncertainties themselves, as discussed in Section 5.5. In Section 6.4.2, the contributions from $\tau$ backgrounds are looked at in different regions of the $K \pi$ mass plot.

At higher mass ( $\gtrsim 1.2 \mathrm{GeV} / c^{2}$ ), where the $\tau$ backgrounds are less, there is some evident excess in the real data over the simulated data. This is likely to be a genuine physics difference: the only $K^{*}$ lineshape used in the simulation is that of $\tau^{-} \rightarrow K^{*}(892)^{-} \nu_{\tau}$ decay whose $p$-wave resonance is generated using a Breit-Wigner amplitude. Higher $K^{*}$ resonances $\left(K^{*}(1410)^{-}, K_{0}^{*}(1430)^{-}, K^{*}(1680)^{-}\right)$, and any possible non-resonant contributions, are not simulated in the Monte Carlo. Consequently, there are no interference terms in the parameterization used in the Monte Carlo. Therefore the excess in the data is likely to be due to any or all of these additional resonances. ALEPH too found similar behaviour at higher $K \pi$ mass [20]. This is discussed further in Section 6.4.3.

Figure 6.10 shows the $K \pi$ mass spectrum from $\tau^{-} \rightarrow K_{S}^{0} \pi^{-} \nu_{\tau}$ decays for the separate $e$-tag and $\mu$-tag samples. In the lower mass region $\left(\lesssim 0.8 \mathrm{GeV} / c^{2}\right)$ there is some excess in the data compared with Monte Carlo, probably due to uncertainties in the $\tau$ backgrounds fed into the simulation. At higher mass $\left(\gtrsim 1.2 \mathrm{GeV} / c^{2}\right)$ there is again some excess in the data which is, at first glance, more pronounced in the electron sample than the muon sample. However, looking at the ratio of the two samples normalised to the sum of weights in each, it seems that the electron and muon samples are entirely consistent, within the statistical uncertainties, over the whole $K \pi$ mass range as shown in Figure 6.11.

\subsection{2 $\tau$ backgrounds as a function of $K \pi$ mass}

Figure 6.12 shows the dominant backgrounds. Overall, the signal $\left(\tau^{-} \rightarrow K_{S}^{0} \pi^{-} \nu_{\tau} ; K_{S}^{0} \rightarrow\right.$ $\pi^{+} \pi^{-}$) purity of the combined sample is $87 \%$. In proportion to the signal, the highest $\tau$ 

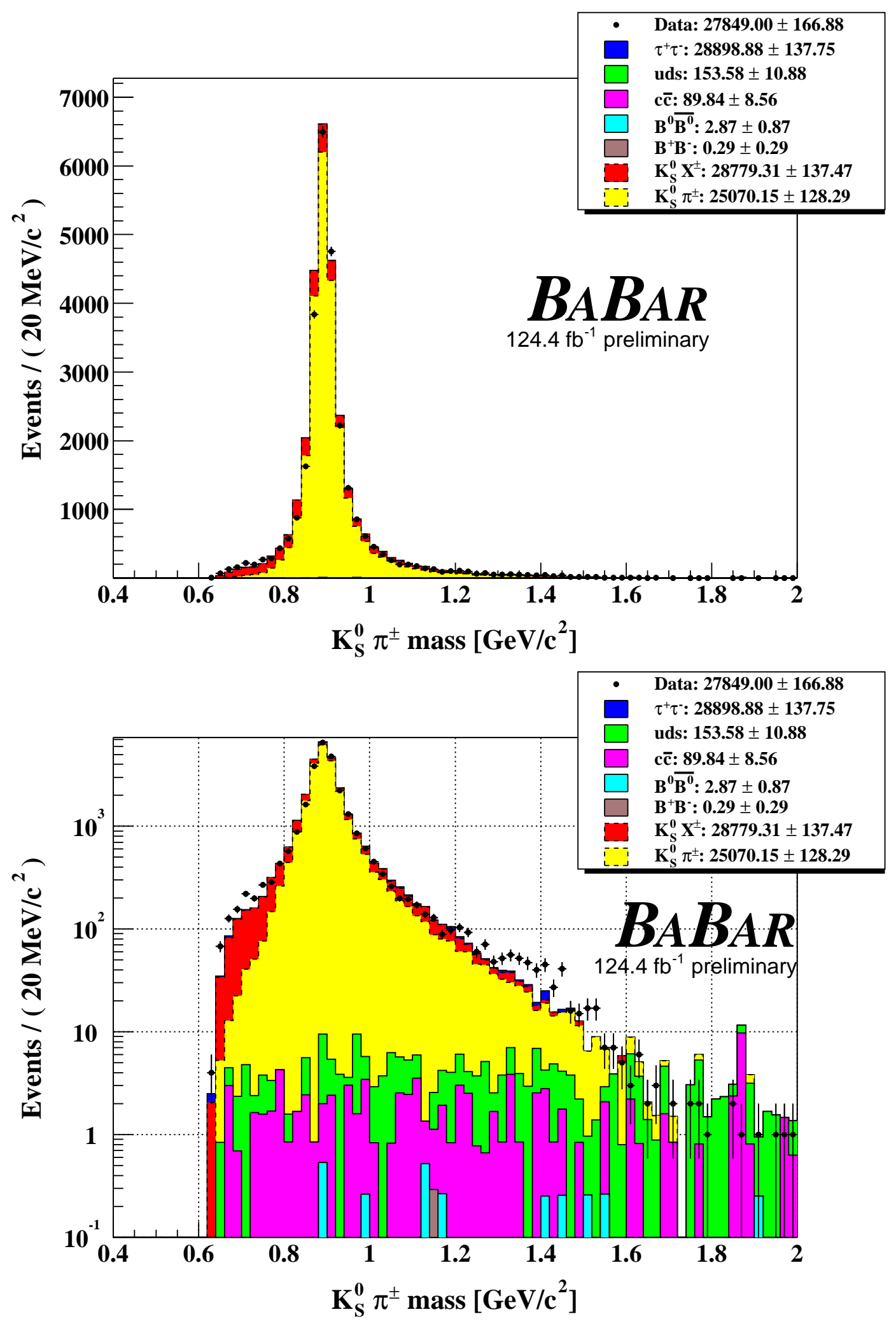

Figure 6.9: Reconstructed $K_{S}^{0} \pi^{ \pm}$mass after selection and efficiency corrections. 

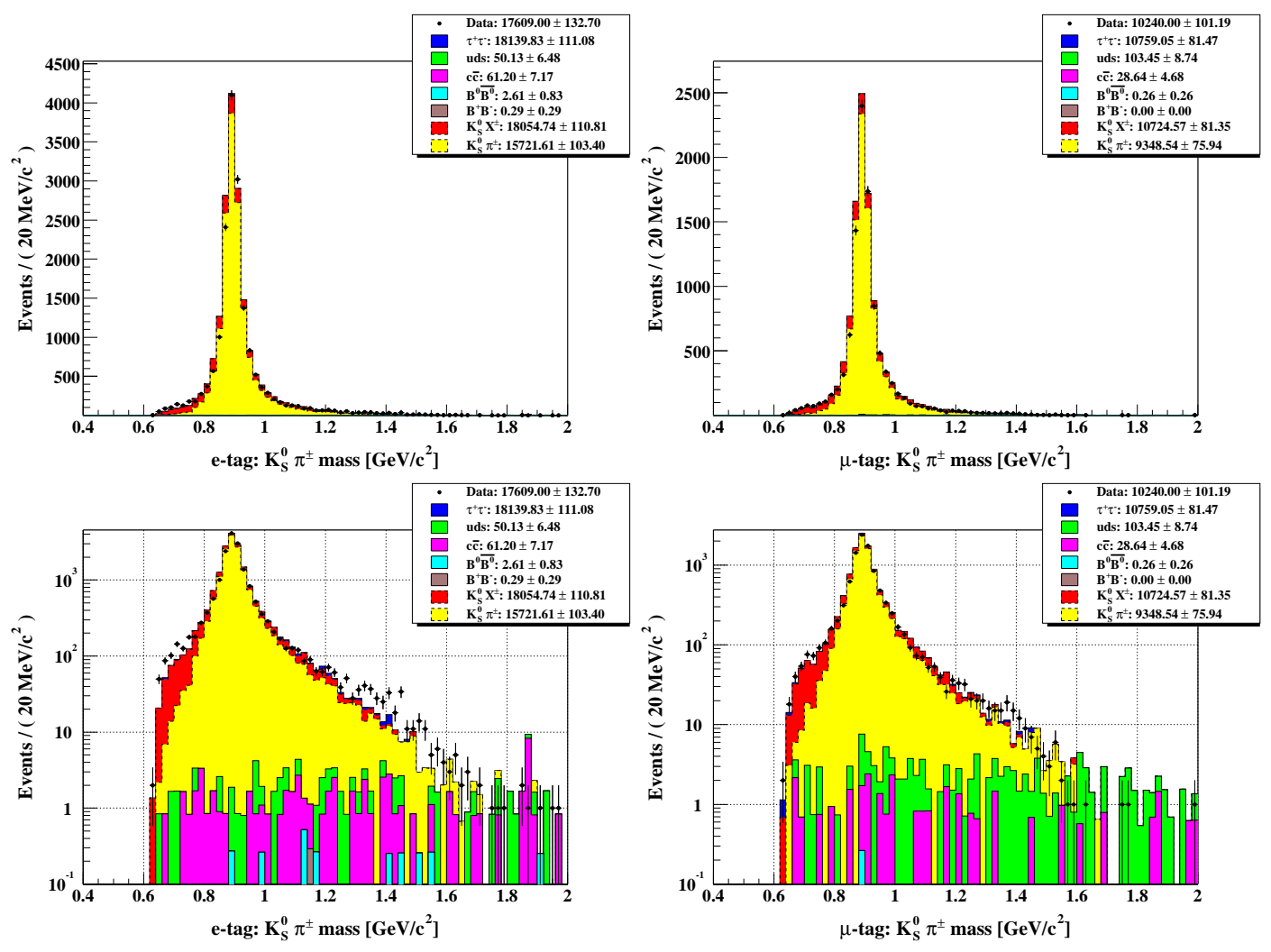

Figure 6.10: Reconstructed $K_{S}^{0} \pi^{ \pm}$mass after selection and efficiency corrections for the e-tag (left) and $\mu$-tag (right) samples.

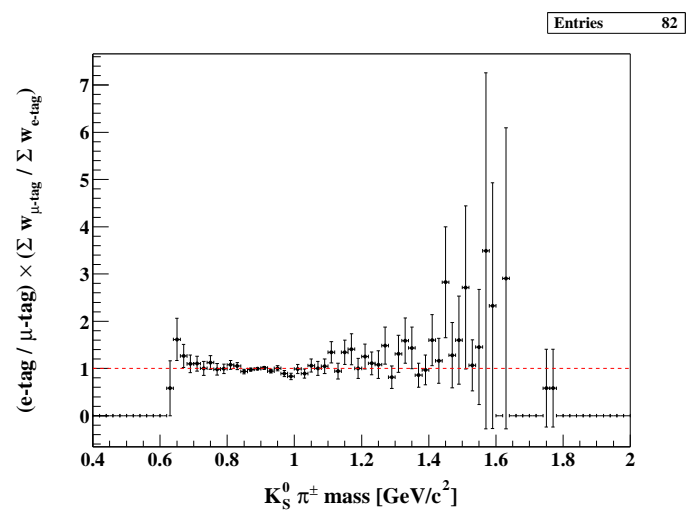

Figure 6.11: Ratio of the numbers of real data events from the e-tag sample divided by the $\mu$-tag sample vs $K_{S}^{0} \pi^{ \pm}$mass, normalised so that the sum of weights $\left(\sum w\right)$ in each sample is the same. Within the statistical uncertainties, the e-tag and $\mu$-tag samples have a ratio of 1 showing that the $K \pi$ mass spectra from the two samples are consistent with one another. 
backgrounds are in the lowest mass bin ${ }^{4}$, i.e. $(-, 0.8]$. In this interval the largest background contribution is from $\tau^{-} \rightarrow K^{0} \pi^{-} \bar{K}^{0} \nu_{\tau}$ (mode 15) decay, and the next largest is $\tau^{-} \rightarrow \pi^{-} \bar{K}^{0} \pi^{0} \nu_{\tau}$ (mode 19). En route to the higher mass region these contributions swap dominance and by the last bin, $(1.2,-)$, there is hardly any $\tau^{-} \rightarrow K^{0} \pi^{-} \bar{K}^{0} \nu_{\tau}$ whatsoever. In each of the four bins from lowest mass to highest mass the signal purities in $\tau$ Monte Carlo are 39\%, 91\%, $74 \%$ and $82 \%$ respectively. In the highest mass bin the largest background contribution is $\tau^{-} \rightarrow \pi^{-} \bar{K}^{0} \pi^{0} \nu_{\tau}$ and the next largest contribution is from $\tau^{-} \rightarrow a_{1}^{-} \nu_{\tau}$ decay which has a branching fraction to a three charged pion final state, as does the signal mode. In the generator, the branching fraction for this decay is $\mathcal{B}\left(\tau^{-} \rightarrow a_{1}^{-} \nu_{\tau} ; a_{1}^{-} \rightarrow \pi^{-} \pi^{-} \pi^{+}\right)=9.19 \%$ (Table B.1). Most of the $a_{1}^{-}$background is, however, cut away by the (signed) $3 \mathrm{D} K_{S}^{0}$ decay length cut $\left(L_{x y z}>1.5 \mathrm{~cm}\right)$ during selection: the $K_{S}^{0}$ in the signal mode has a reasonably long lifetime and so creates a displaced vertex as a handle to cut away primary vertices ${ }^{5}$. The relative proportion of $\tau^{-} \rightarrow K^{-} K^{0} \nu_{\tau}$ (mode 22) to signal is reasonably constant over all the $K \pi$ mass bins.

\subsubsection{Higher $K^{*}$ resonances}

The dominance of the $K^{*}(892)^{-}$resonance in the $K \pi$ mass spectrum is stark, as shown in Figure 6.9. Still, the mass resolution is good enough in the tails to suggest that there is some excess mass above the expected $K^{*}(892)^{-}$tail. One proposal is a contribution from the $K^{*}(1410)^{-}$vector state, which could exhibit $K^{*}(892)-K^{*}(1410)$ interference in an analogous manner to $\rho(770)-\rho(1450)$ in the $\pi^{-} \pi^{0}$ system, observations of which have been reported $[15,59]$. Our preliminary study and interpretation of the $p$-wave contributions to the $K \pi$ mass spectrum is given in Section 6.4.3.2.

The search for $s$-wave (longitudinal) contributions to the $K \pi$ mass spectrum is discussed

\footnotetext{
${ }^{4}$ Notation: (, is used to denote a non-inclusive interval limit; [, denotes an inclusive interval limit; -, means that there has been no restriction placed on that part of the interval (although the bins might run out, but that is a different issue).

${ }^{5}$ The $a_{1}^{-}$effectively comes from the IP since the lifetimes of the $\tau$ and the $a_{1}^{-}$are so short.
} 

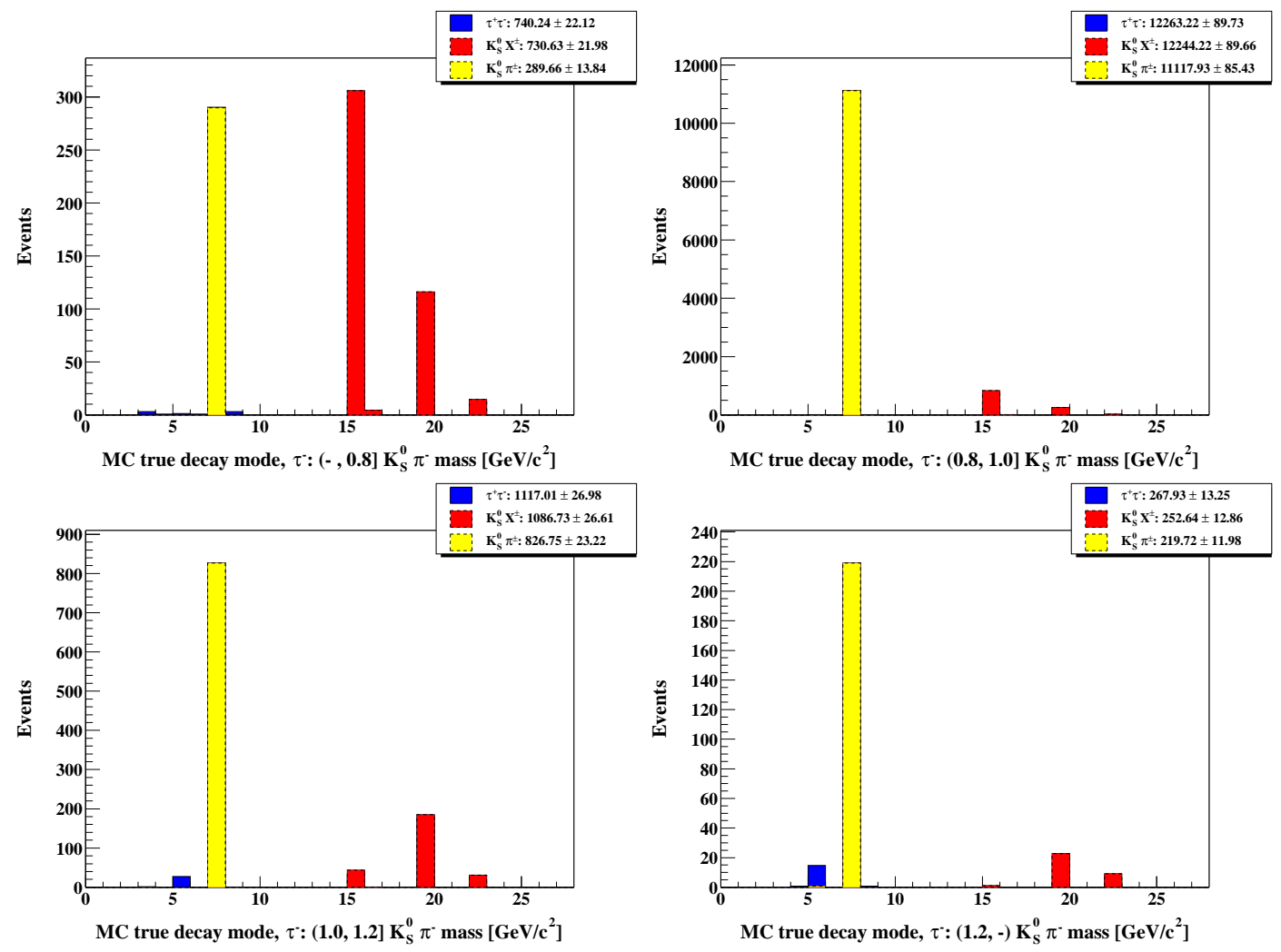

Figure 6.12: Signal (yellow) and $\tau$ backgrounds (other colours) in four $K \pi$ mass bins: $(-, 0.8],(0.8,1.0],(1.0,1.2],(1.2,-)$, where all masses are in $\mathrm{GeV} / c^{2}$ units. The $\tau$ backgrounds, in proportion to the signal, are highest in the lowest mass bin, i.e. $(-, 0.8]$. Shown for simulated $\tau^{-}$decays with each bin corresponding to a particular decay mode (Table B.1). 
in Section 6.4.3.3.

\subsubsection{Parameterization of the $K \pi$ mass spectrum}

The $K \pi$ final state is made via the weak vector current. Theoretically, the decay rate for $\tau^{-} \rightarrow(K \pi)^{-} \nu_{\tau}$ relative to the electronic width is given by [144]:

$$
\frac{\Gamma_{K \pi}}{\Gamma_{e}} \sim \int \frac{\mathrm{d} s}{m_{\tau}^{2}}\left(1-\frac{s}{m_{\tau}^{2}}\right)^{2}\left(1+2 \frac{s}{m_{\tau}^{2}}\right)\left(s-m_{+}^{2}\right)^{3 / 2}\left(s-m_{-}^{2}\right)^{3 / 2} s^{-3}\left|f_{1}(s)\right|^{2},
$$

where $m_{ \pm}=m_{K} \pm m_{\pi}$. The vector form factor is given by:

$$
f_{1}(s)=\frac{1}{1+\beta+\delta+\cdots}\left[B W_{K^{* \prime}}(s)+\beta B W_{K^{* \prime \prime}}(s)+\delta B W_{K^{* \prime \prime \prime}}(s)+\cdots\right],
$$

similar to the Kühn-Santamaria model [57] for pions, assuming real $\beta, \delta$ and a set of vector $K^{*}$ resonances. The Breit-Wigner propagators have the form:

$$
B W_{X}(s)=\frac{m_{X}^{2}}{m_{X}^{2}-s-i \sqrt{s} \Gamma(s)},
$$

where $X$ represents the applicable two meson resonance. The energy dependent width, $\Gamma(s)$, is given by:

$$
\begin{gathered}
\Gamma(s)=\Gamma_{X} \frac{m_{X}^{2}}{s}\left(\frac{p(s)}{p\left(m_{X}^{2}\right)}\right)^{2 L+1} ; \\
p(s)=\frac{1}{2 \sqrt{s}} \sqrt{\left(s-m_{+}^{2}\right)\left(s-m_{-}^{2}\right)},
\end{gathered}
$$

where $L$ is the $K-\pi$ orbital angular momentum, i.e. $L=1$ for $p$-wave.

This model was refined to include the scalar contribution in the parameterization [74]. The corresponding scalar form factor when there are two vector and one scalar resonance is given by:

$$
\begin{aligned}
f_{0}(s) & =\frac{m_{K}^{2}-m_{\pi}^{2}}{s} \frac{c_{1}}{1+\beta}\left[\frac{m_{K^{* \prime}}^{2}-s}{m_{K^{* \prime}}^{2}} B W_{K^{* \prime}}(s)+\frac{m_{K^{* \prime \prime}}^{2}-s}{m_{K^{* \prime \prime}}^{2}} B W_{K^{* \prime \prime}}(s)\right] \\
& +\frac{m_{K}^{2}-m_{\pi}^{2}}{m_{K_{0}^{*}}^{2}} c_{0} B W_{K_{0}^{*}}(s) \\
& \simeq\left(m_{K}^{2}-m_{\pi}^{2}\right)\left[\frac{c_{1}}{s}+\frac{c_{0}}{m_{K_{0}^{*}}} B W_{K_{0}^{*}}(s)\right]
\end{aligned}
$$


where $c_{1}$ and $c_{0}$ determine the strengths of the vector and scalar resonances respectively. $c_{0}$ is a number of order 1 since the coefficient proportional to the symmetry breaking has been factored out ${ }^{6}$.

\subsubsection{2 $K \pi p$-wave results}

Figure $6.13 a$ shows a fit to our background-subtracted $K \pi$ mass spectrum using the same parameterization as ALEPH [20]. This model assumes $K^{*}(892)-K^{*}(1410)$ interference. The form factor used is given by inserting these vector resonance contributions into Eq. (6.5) and the fit returns $\beta=-0.076 \pm 0.015$. For comparison, the ALEPH fit is shown in Figure 6.13b.
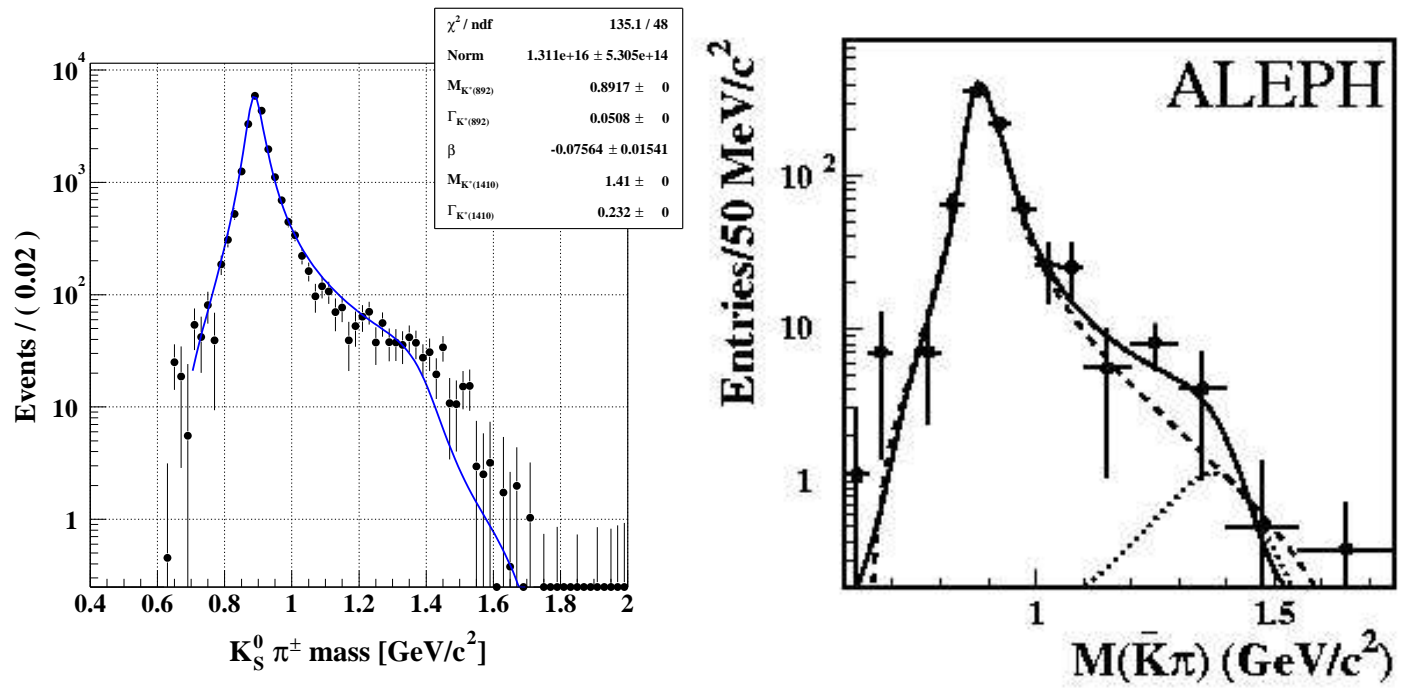

Figure 6.13: a) Fit to our background-subtracted $K \pi$ mass data using the $K^{*}(892)-K^{*}(1410)$ interference in the parameterization used by ALEPH (left). b) The result obtained by ALEPH fitting to the K $\pi$ mass spectrum [20] (right).

Our $K \pi$ mass spectrum benefits from higher statistics and therefore has more significance in the higher $K \pi$ mass region, where statistics are a premium, than has been achieved previously. It can be clearly seen from our distribution that a $K^{*}(1410)$ contribution

\footnotetext{
${ }^{6}$ In the $S U(3)_{f}$ limit, where $m_{K}=m_{\pi}$, the scalar contribution is zero.
} 
seems insufficient to explain the excess in mass over the $K^{*}(892)$ tail. One or both of these two possibilities seem the most plausible shortfalls in the model:

$\diamond$ The amplitude and phase cannot be varied separately.

$\diamond$ There is a contribution from the vector $K^{*}(1680)$ resonance, that is distorted because of the end of the phase space.

$\diamond$ There is a contribution from the scalar $K_{0}^{*}(1430)$ resonance.

In fact, there is some room for doubt about the extent to which the $K \pi$ mass spectrum is affected by the $K^{*}(1410)$ at all. In analogy with $\rho(1450)$, another proposal [145] is that the same kind of interference pattern as that from an interfering $\rho(1450)$ can be generated with a $\rho^{\prime}$ at $\sim 1600 \mathrm{MeV} / c^{2}$ and width of $\sim 400 \mathrm{MeV} / c^{2}$ [146]. The reason for doubting the $\rho(1450)$ as the interference generator, particularly in $\tau$ decay, is that the parameters used in the model stem from $\pi \pi$-scattering results which have interference information from the other waves to define phase as well as amplitude; the pion form factor in $\tau$ decay only has information on intensity if pure $p$-wave, and the corresponding mass and width parameters are sensitive to the assumptions made about the relative phase. In $J / \psi \rightarrow$ $\pi^{+} \pi^{-} \pi^{0}$ studies [146], each dipion was studied as if in a $p$-wave state, but the phase information was retrieved using Bose symmetry. The resulting mass and width values for the $\rho^{\prime}$ were in agreement with the original $\pi \pi$-scattering experiments. The interpretation given in [146] is that the $\rho^{\prime}$ is the $1^{-}$partner of the spin $3 \rho(1690)$ in the $d$-wave $q \bar{q}$ triplet ground state, where the quark spins add to 1 so that, with the $L=2 d$-wave orbital angular momentum, it is possible to make states of $J=3,2,1$ with negative $C$ and $P$.

For $1^{-}$strange meson states, the partner of the spin $3 K^{*}(1780)$ which would correspond to the $\rho^{\prime}$ would be the $K^{*}(1680)$. A suggestion is, therefore, to construct the $p$-wave amplitude as a coherent sum of the $K^{*}(892)$ and $K^{*}(1680)$ amplitudes with a relative strength and relative phase as parameters in the fit [145]. Maybe then destructive interference effects could yield the distribution we see. Since the $K^{*}(1410)$ couples 
only weakly to $K \pi, \mathcal{B}\left(K^{*}(1410) \rightarrow K \pi\right) \sim 7 \%$, it almost certainly cannot produce the behaviour we observe because of its low mass. Replacing it with the $K^{*}(1680)$, $\mathcal{B}\left(K^{*}(1680) \rightarrow K \pi\right) \sim 39 \%^{7}$, and allowing the relative amplitude to be a free parameter, yields a better fit to the data, Figure 6.14. In this case the fit returns $\beta=-0.079 \pm 0.013$ and $\beta=-0.059 \pm 0.010$ when using the PDG and LASS results respectively as input.
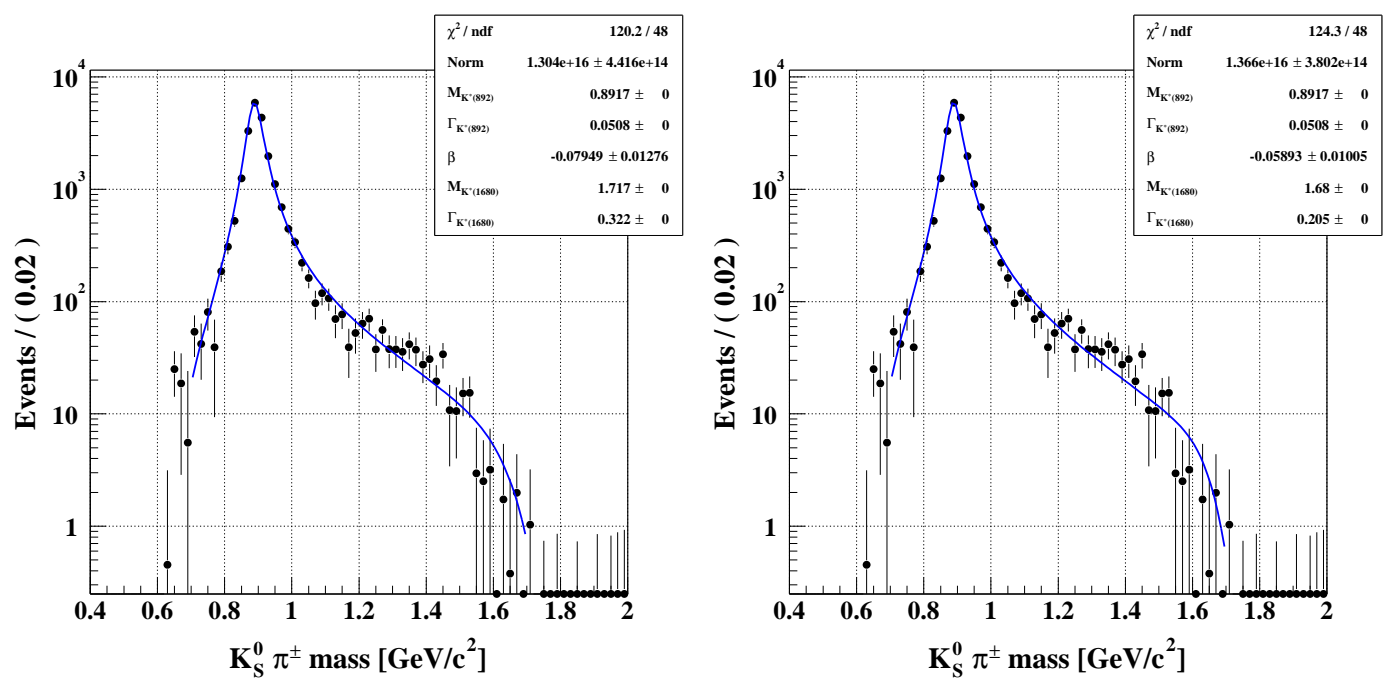

Figure 6.14: Fit to our background-subtracted $K \pi$ mass data using the $K^{*}(892)-K^{*}(1680)$ interference model. The PDG masses and widths are used to fix the masses and width of the $K^{*}$ resonances (left), except for $K^{*}(1680)$ where the LASS values have been used (right). There is very little difference.

Using a three vector resonance interference model, $K^{*}(892)-K^{*}(1410)-K^{*}(1680)$, does not produce any significant improvement upon the $K^{*}(892)-K^{*}(1680)$ interference model, which adds support to the claim that the $K^{*}(1410)$ contribution is small (or nonexistent). The $K^{*}(892)-K^{*}(1410)-K^{*}(1680)$ interference fit is shown in Figure 6.15. The values $\beta=-0.017 \pm 0.017, \delta=-0.075 \pm 0.014$ and $\beta=-0.028 \pm 0.017, \delta=$ $-0.055 \pm 0.011$ are returned when using the PDG and LASS results respectively as input.

Even though the fit to the $K \pi$ mass spectrum improves with a $K^{*}(1680)$ component, it is still not perfect. There could be a number of reasons for this which would merit

\footnotetext{
${ }^{7}$ Not only does $K^{*}(1680)$ have a branching ratio to $K \pi$, but it also has fractions to $K \rho(\sim 31 \%)$ and $K^{*}(892) \pi(\sim 30 \%)$ which could actually be partially reconstructed by our selection procedure.
} 

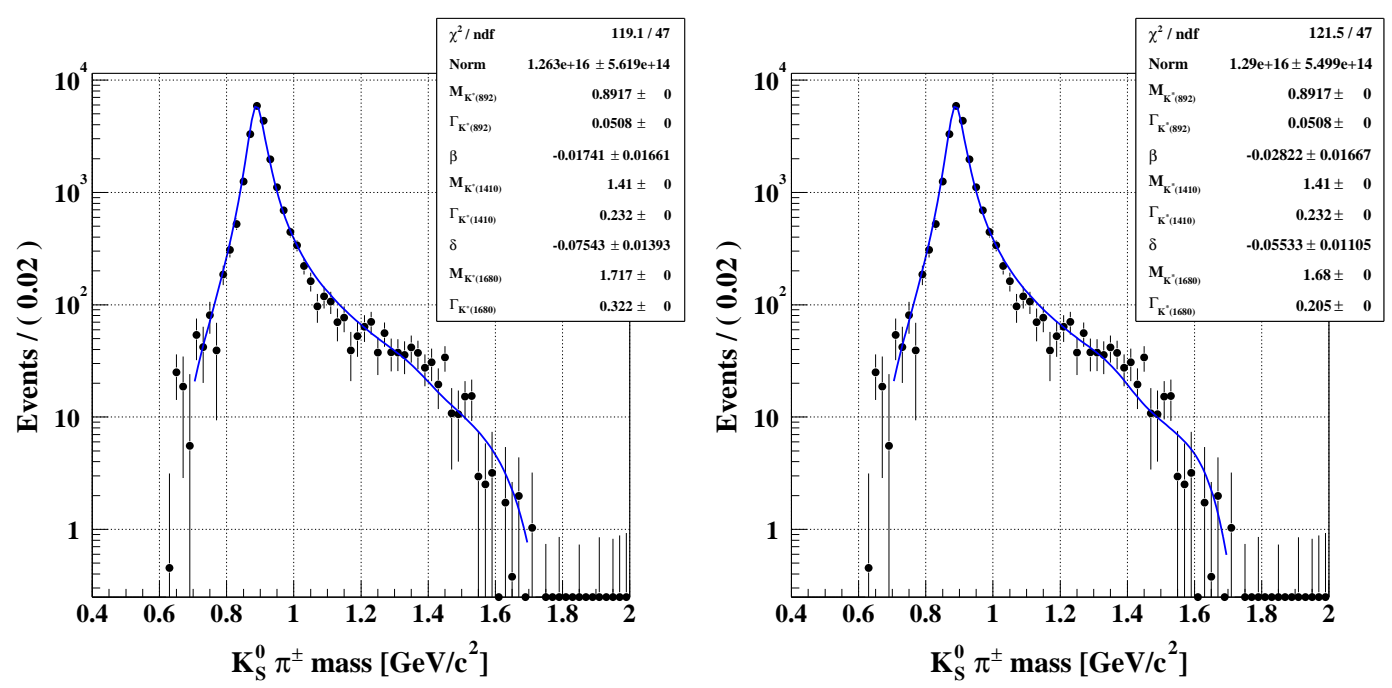

Figure 6.15: Fit to our background-subtracted $K \pi$ mass data using the $K^{*}(892)-K^{*}(1410)-K^{*}(1680)$ interference model. The PDG values are used to fix the masses and width (left), except for $K^{*}(1680)$ where the LASS values have been used (right).

further study. Firstly, there has been no inclusion of any $s$-wave component to the parameterization. Longitudinal contributions from a possible $K_{0}^{*}(1430)$ resonance might cause additional interference that we currently do not model. Eq. (6.9) suggests a parameterization for the scalar form factor. However, since we can only find a hint of a scalar contribution in our data (Section 6.4.3.3) we feel that we cannot justify applying this model at this stage. Moreover, results from $K \pi$-scattering experiments such as LASS give doubt as to the applicability of a Breit-Wigner PDF in the case of $s$-wave $[61,62]$. The second reason could be that no attempt has been made to unfold the detector effects in this analysis. In the fits we make, unsmeared Breit-Wigner PDFs are used to describe the vector resonances; their masses and widths are fixed to the PDG values. In making a background-subtracted plot as we do, we use (smeared) Monte Carlo as a way of subtracting the non- $K^{*}$ background from the real data. However, the peak in the data is still smeared by detector effects, whereas the fitted curves are not. 


\subsubsection{3 $K \pi s$-wave results}

Longitudinal contributions to the strange spectral functions are predicted by theory and so, in principle, should be observable in the $K \pi$ mass spectrum. The theoretical importance of the longitudinal contributions was discussed in Section 2.4.9. In $\tau^{-} \rightarrow(K \pi)^{-} \nu_{\tau}$ decay, the resonance expected to deliver the $s$-wave contribution is the $K_{0}^{*}(1430)$, which has not yet been observed in hadronic $\tau$ decay data. In fact, even theoretically its branching ratio in $\tau$ decay is expected to be small: $\mathcal{B}\left(\tau^{-} \rightarrow K_{0}^{*}(1430)^{-} \nu_{\tau}\right) \sim 6 \times 10^{-5}$, Eq. (2.41).

Since in our $K \pi$ mass spectrum we can see an excess in mass above the $K^{*}(892)$ tail, there may be some contribution from the $K_{0}^{*}(1430)$. In order to assess any possible $s$ wave component, it is necessary to try to separate it from the other ( $p$-wave) resonances that are in the $\tau^{-} \rightarrow(K \pi)^{-} \nu_{\tau}$ phase space. An attempt to do this has been made here by appealing to the difference in $J^{P}$. We first define the $K^{*}$ helicity cosine $\left(\cos \Psi_{\text {hel }}^{K_{S}^{0}}\right)$ as the cosine of angle between the $K_{S}^{0}$ from the $K^{*}$ in the $K^{*}$ rest frame and the LAB momentum vector of the $K^{*}$. Since there is still a fair amount of non- $K^{*}$ background in the $K \pi$ mass distribution, rather than looking at the forward-backward (FB) asymmetry, as defined by whether the $K_{S}^{0}$ from the decaying $K^{*}$ has a positive or negative helicity cosine, it was decided to weight each event by a normalised Legendre polynomial weight. The reason the original FB asymmetry idea was dropped is because background contributions are present in both numerator and denominator, which makes background subtraction difficult. So, a weighting scheme is chosen to see the difference between real and simulated data. We weight the $K \pi$ mass spectrum with weights corresponding to the normalised Legendre polynomials ${ }^{8}$ [147]:

$$
\begin{aligned}
Y_{0}\left(\cos \Psi_{\text {hel }}^{K_{S}^{0}}\right) & =\sqrt{\frac{1}{2}} ; \\
Y_{1}\left(\cos \Psi_{\text {hel }}^{K_{S}^{0}}\right) & =\sqrt{\frac{3}{2}} \cos \Psi_{\text {hel }}^{K_{S}^{0}} ; \\
Y_{2}\left(\cos \Psi_{\text {hel }}^{K_{S}^{0}}\right) & =\sqrt{\frac{5}{2}}\left(\frac{3}{2} \cos ^{2} \Psi_{\text {hel }}^{K_{S}^{0}}-\frac{1}{2}\right) .
\end{aligned}
$$

\footnotetext{
${ }^{8}$ Legendre polynomials [147]: $P_{0}(x)=1 ; P_{1}(x)=x ; P_{2}(x)=\frac{1}{2}\left(3 x^{2}-1\right)$.
} 
These are such that

$$
\int_{-1}^{1} Y_{i}^{2} \mathrm{~d}\left(\cos \Psi_{\text {hel }}^{K_{S}^{0}}\right)=1
$$

and $Y_{i}$ is orthogonal to $Y_{j}(i \neq j)$. Therefore, for a distribution with $N$ events which is quadratic in $\cos \Psi_{\mathrm{hel}}^{K_{S}^{0}}$, we can write

$$
\frac{\mathrm{d} N}{\mathrm{~d}\left(\cos \Psi_{\mathrm{hel}}^{K_{S}^{0}}\right)}=N\left\{\left\langle Y_{0}\right\rangle Y_{0}+\left\langle Y_{1}\right\rangle Y_{1}+\left\langle Y_{2}\right\rangle Y_{2}\right\},
$$

where

$$
\begin{aligned}
N\left\langle Y_{i}\right\rangle & =\int_{-1}^{1} Y_{i} \frac{\mathrm{d} N}{\mathrm{~d}\left(\cos \Psi_{\mathrm{hel}}^{K_{S}^{0}}\right)} \mathrm{d}\left(\cos \Psi_{\text {hel }}^{K_{S}^{0}}\right) \\
& \simeq \sum_{k=1}^{N} Y_{i}\left[\left(\cos \Psi_{\text {hel }}^{K_{S}^{0}}\right)_{k}\right] .
\end{aligned}
$$

With this choice of normalisation, $N\left\langle Y_{i}\right\rangle$ is plotted in each mass bin, i.e. the number of events in the bin times the average value of $Y_{i}$ for that bin. This is sometimes referred to as the "unnormalised $Y_{i}$ moment". Clearly, dividing by $N$ gives $\left\langle Y_{i}\right\rangle$, and this is called the "normalised $Y_{i}$ moment". The normalised $Y_{i}$ moment is therefore the average value of $Y_{i}$ for the bin in question. Using these definitions helps to keep proper track of the relative strength of the different contributions.

With these definitions in place, and based on the LASS observation (Figure 6.16), we can speculate on what we should see if there is indeed $s p$-interference in our data. (The LASS observation is a projection of any $\cos \Psi_{\mathrm{hel}}^{K_{S}^{0}}$ dependence and therefore corresponds to the data weighted with the $Y_{1}$ weight in our notation.) Any real $s p$-interference is proportional to the cosine of the $s-p$ phase difference. The $s$-wave phase should be changing slowly near the $K^{*}(892)$ resonance, while the $p$-wave phase changes rapidly; $p-s$ should be $\sim 0^{\circ}$ at $\sim 850 \mathrm{MeV} / c^{2}$, going through $90^{\circ}$ at $\sim 920 \mathrm{MeV} / c^{2}$, and increasing after that. It follows that the cosine sum should change from positive to zero to negative in going through this region if there is any true $s p$-interference. (The observed overall sign might be different, but it is the rapid change with mass which is important.) In order to argue that an $s$-wave contribution is present in our data we need to observe some such effect. 
Chapter 6. Studies of $\tau^{-} \rightarrow K_{S}^{0} \pi^{-} \nu_{\tau}$ decay

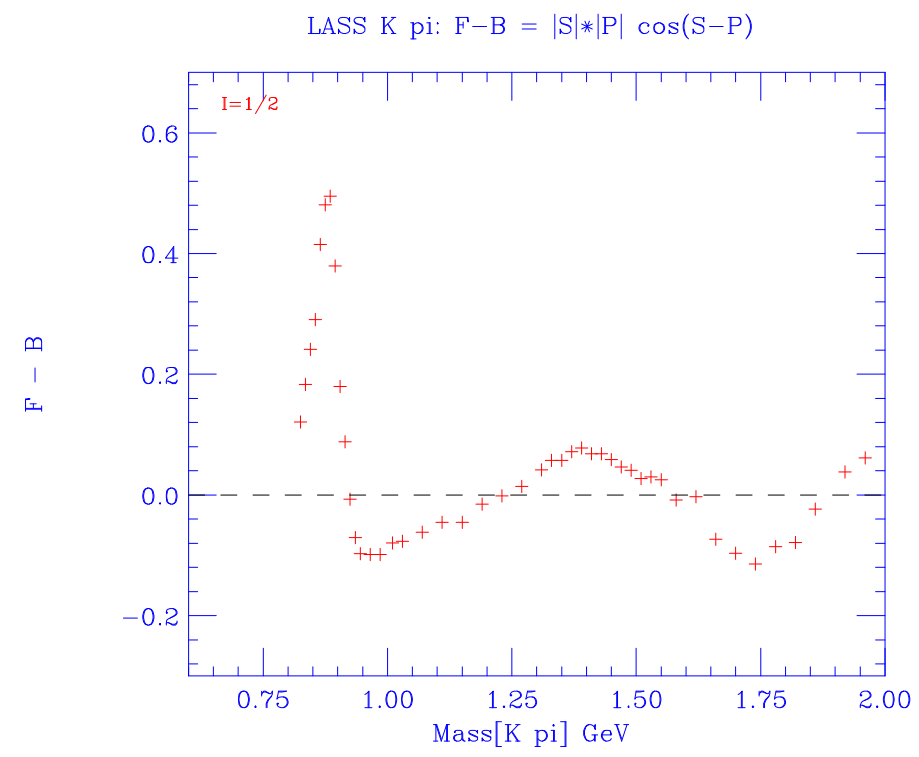

Figure 6.16: Using the LASS amplitude and phase results for $K \pi$ elastic scattering, the forward-backward asymmetry as a function of $K \pi$ mass is plotted [148]. This is essentially comparable with a $Y_{1}$-weighted plot in our notation, i.e. $\propto \cos \Psi_{\text {hel }}^{K_{S}^{0}}$. (Error bars not shown: they are tiny compared to those we obtain from $\tau^{-} \rightarrow(K \pi)^{-} \nu_{\tau}$ data. $)$

Figure 6.17 shows plots of the $Y_{0}, Y_{1}$ and $Y_{2}$-weighted $K \pi$ mass spectrum for our realminus-simulated data spectrum. Since there is no $s p$-interference modelled in the simulation, we would expect the $Y_{1}$-weighted distribution to be flat if there were no real $s p$-interference. Qualitatively, however, our $Y_{1}$-weighted distribution looks the same as Figure 6.16: there is a suggestion of $s p$-interference in our data. That said, at the level of statistics we have available at higher $K \pi$ mass, it is difficult to be convinced that this is not just either a statistical effect or a systematic problem since the normalisation between the data and Monte Carlo in making the real minus simulated data subtracted plot is, at the level of precision we demand, not the same. For example, the $Y_{2}$ weight should project out the $\cos ^{2} \Psi_{\text {hel }}^{K_{S}^{0}}$ dependence. Since there is a clear non-zero point in the $K^{*}(892)$ region we can see that we are not getting the normalisation perfect between the real data and Monte Carlo: this distribution should be zero there.

In this analysis we do not reach a conclusive result as to whether there is any longitudinal 
Chapter 6. Studies of $\boldsymbol{\tau}^{-} \rightarrow \boldsymbol{K}_{S}^{0} \pi^{-} \boldsymbol{\nu}_{\boldsymbol{\tau}}$ decay
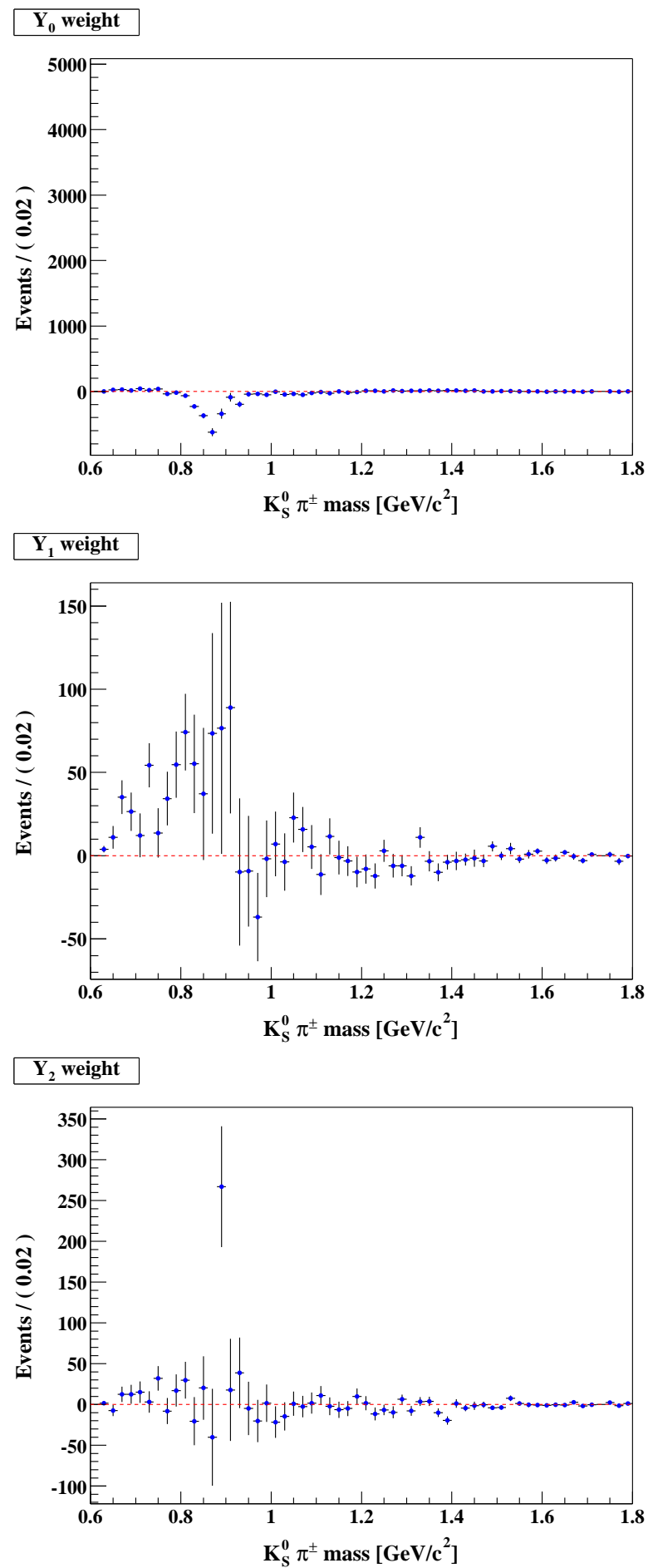

Figure 6.17: BABAR real-minus-simulated data weighted with the normalised Legendre polynomials (Eq. (6.10)). Qualitatively, the $Y_{1}$-weighted distribution shows a possible sp-interference effect; it is similar to the LASS observation, Figure 6.16. The $Y_{0}$ and $Y_{2}$ distributions suggest that our normalisation (used to perform the background subtraction) is not perfect. 
(s-wave) contribution to $\tau^{-} \rightarrow(K \pi)^{-} \nu_{\tau}$ decay. This is an avenue that would benefit from further study: theoretically these contributions cause problems in the OPE used to calculate $R_{\tau}$ from which $m_{s}$ and $\left|V_{u s}\right|$ are extracted. An experimental measurement of the $K \pi s$-wave spectral function (i.e. invariant mass spectrum) would enable theorists to perform the longitudinal subtraction they need to improve the measurements of $m_{s}$ and $\left|V_{u s}\right|$.

\subsection{Summary and outlook}

The aim of this analysis has been to study the decay $\tau^{-} \rightarrow K_{S}^{0} \pi^{-} \nu_{\tau}\left(K_{S}^{0} \rightarrow \pi^{+} \pi^{-}\right)$using the $B A B A R$ detector. Using $124.4 \mathrm{fb}^{-1}$ of data we present the preliminary result

$$
\mathcal{B}\left(\tau^{-} \rightarrow \bar{K}^{0} \pi^{-} \nu_{\tau}\right)=(0.830 \pm 0.005 \text { (stat) } \pm 0.042 \text { (syst) }) \%,
$$

which is the world's most precise measurement to date of this branching ratio, and is consistent with the PDG value [39]. This result, unlike most of the $\mathcal{B}\left(\tau^{-} \rightarrow \bar{K}^{0} \pi^{-} \nu_{\tau}\right)$ measurements currently listed in the PDG, is systematics dominated and so the biggest future improvement to this number should come from reducing the systematic uncertainties in the analysis.

In this work we have measured the $K \pi$ invariant mass distribution using $\tau^{-} \rightarrow K_{S}^{0} \pi^{-} \nu_{\tau}$ decays. This needs to be combined with results from the other decay channels with an overall net change of strangeness in order to compute the total strange spectral function which is necessary to calculate $R_{\tau}^{\text {strange }}$. Using the higher statistics data samples available at $B A B A R$ it is expected that a more precise value of $R_{\tau}^{\text {strange }}$ will be obtained than in previous experiments. Since $R_{\tau}^{\text {strange }}$ is used in the FESR extraction of $m_{s}$, smaller uncertainties associated with $R_{\tau}^{\text {strange }}$ will reduce the uncertainty on $m_{s}$.

Moreover, the $K \pi$ mass distribution we observe reveals excess contributions at higher $K \pi$ mass above the $K^{*}(892)$ tail. Whilst in the past this has been thought to be due to $K^{*}(892)-K^{*}(1410)$ interference, we find that the $K^{*}(1410)$, whose branching ratio to 
$K \pi$ is approximately $7 \%$, seems insufficient to explain the excess mass observed in the data. Instead, we perform a fit using a $K^{*}(892)-K^{*}(1680)$ interference model and find better agreement. The discrepancy that remains could be due to an $s$-wave contribution to the interference that is not parameterized in the model used, and/or detector smearing that is not accounted for in our fit. However, our search for an $s p$-interference effect has only whet the appetite for further study: qualitatively we see an interference effect similar to LASS, but we have neither the confidence in the statistics nor systematics at the level of precision needed to announce an observation in hadronic $\tau$ decay data with an overall net change of strangeness. Any $s$-wave component is expected to be solely due to a $K_{0}^{*}(1430)^{-}$resonance which decays mostly to $K \pi$. To date this resonance has not been observed in $\tau$ decay data, but any possible future measurement of its branching ratio and subsequent translation of the $s$-wave invariant mass spectrum into the $J=0$ spectral function would significantly improve the FESR extraction of $m_{s}$ by enabling theorists to employ a longitudinal subtraction technique.

In conclusion, both reducing the uncertainties in the higher $K \pi$ mass region and measuring the $s$-wave contribution to $K \pi$ data will be of benefit to theory, particularly the extraction of $m_{s}$ and $\left|V_{u s}\right|$ from $R_{\tau}$. 


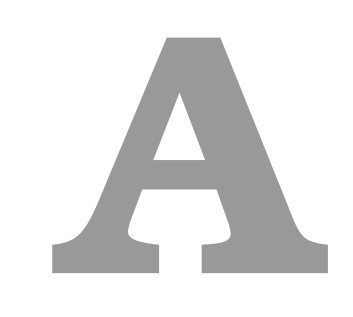

\section{Sensitivity of the OPE integrals to $m_{s}$}

The information contained below was kindly provided by Kim Maltman [149] and is included here for completeness.

\section{A.1 Outline}

Maltman's information is based on the integrated OPE information for the flavour $u d-u s$ difference that can be handled with reasonable to good reliability theoretically using the general FESR relation, Eq. (2.29). The OPE results quoted correspond to the RHS of this relation, for the $\Delta \prod_{V+A}^{(0+1)}(s) \equiv \prod_{V+A ; u d}^{(0+1)}(s)-\prod_{V+A ; u s}^{(0+1)}(s)$ correlator difference. The spectral integrals quoted below are the flavour $u d$ part of the corresponding difference on the LHS. Comparing the OPE integrals (RHS) with the $u d$ part of the LHS shows the impact of $m_{s}$ on the spectral integral since the difference is produced by the difference between $m_{s}$ and $\left(m_{u}+m_{d}\right) / 2$, and contributions associated with the light quark $(u, d)$ masses are negligible. 
Maltman also includes some information about reliability of the OPE representation since some apparent claims in the literature about the $(0,0)$ spectral weight case can be misleading. Rather than being the most reliable weight theoretically, Maltman suggests that it exhibits rather "dicey" convergence behaviour and that better weights can be found.

\section{A.2 Input values}

The integrated OPE results for the $\Delta \Pi_{V+A}^{(0+1)}(s) \equiv \prod_{V+A ; u d}^{(0+1)}(s)-\Pi_{V+A ; u s}^{(0+1)}(s)$ difference as a function of $m_{s}(2 \mathrm{GeV})$ are based on the following input:

1. $\alpha_{S}=0.334$.

2. $m_{s}(2 \mathrm{GeV})=(110 \pm 25) \mathrm{MeV}$ (range suggested by all current lattice and sum rule analyses), with 4-loop running for $\alpha_{S}\left(Q^{2}\right)$ and $m_{s}\left(Q^{2}\right)$ based on the input $\alpha_{S}$ value in (1).

3. $\langle s \bar{s}\rangle /\langle u \bar{u}\rangle=0.85$. Needed for $D=4$ contributions. The uncertainty is not large, and this uncertainty does not play any significant role. Remember that the gluon condensate contribution does not enter the flavour breaking difference.

4. $\rho_{\mathrm{VSA}}=1$, i.e. VSA for the $D=64$-quark VEVs (even if this is inaccurate by a large factor the $D=6$ contributions are still tiny compared to the dominant $D=2$ terms proportional to $m_{s}^{2}$ ).

5. Define $a \equiv \alpha_{S} / \pi$. The known OPE $D=2$ coefficients out to $\mathcal{O}\left(a^{2}\right)$, and an estimate for the $\mathcal{O}\left(a^{3}, a^{4}\right)$ coefficients [150], which are based on the PMS, FAC methods, and tested against known $\mathcal{O}\left(a^{3} n_{f}^{2}, a^{3} n_{f}, a^{4} n f^{3}, a^{4} n_{f}^{2}\right)$ contributions. The BCK02 $\mathcal{O}\left(a^{3}, a^{4}\right)$ coefficients [151] have been used in getting the $\mathcal{O}\left(a^{3}, a^{4}\right)$ integrated terms here. (The tabulated values in the summary use only up to the known terms, but some additional material about the bad convergence behaviour of the 
$(0,0)$ "kinematic" weight is included after the summary, and this information is relevant there.)

6. All $D=2$ and $D=4 \mathrm{OPE}$ contributions are evaluated using the contour improvement integration method.

\section{A.3 Results}

Results for two cases are presented:

1. The $(0,0)$ or "kinematic" weight case $\left(1-y_{\tau}\right)^{2}\left(1+2 y_{\tau}\right)$, where $y_{\tau}=s / s_{0}$, and $s_{0}$ is the endpoint of the corresponding spectral integral,

$$
\int_{0}^{s_{0}} \mathrm{~d} s \rho(s) w(s) \quad\left[\mathrm{GeV}^{2}\right],
$$

with $\Delta \rho_{V+A}^{(0+1)}(s) \equiv \rho_{V+A ; u d}^{(0+1)}(s)-\rho_{V+A ; u s}^{(0+1)}(s)$ and using natural units (i.e. $\left.c=1\right)$. Only the $J=(0+1)$ sum part of the spectral function difference is used because the other part $\left(J=0\right.$, weighted by $\left.w_{L}\left(y_{\tau}\right)=-2\left(y_{\tau}\right)\left(1-y_{\tau}\right)^{2}\right)$, is exceptionally badly behaved on the OPE side and cannot be dealt with. When $s_{0}=m_{\tau}^{2}$, one gets the $R_{\tau}$ type integral, up to constant factors.

2. $w_{10}\left(y_{\tau}\right)=1-y_{\tau}-y_{\tau}^{2}+2 y_{\tau}^{5}-y_{\tau}^{8}-y_{\tau}^{9}+y_{\tau}^{10}$, a weight designed by Maltman and Kambor [35] to have both less $u d-u s$ cancellation in the spectral integrals (hence smaller relative errors than the $(0,0)$ case) and better convergence behaviour of the integrated OPE $D=2$ series.

The integrated OPE for any input $m_{s}(2 \mathrm{GeV})$ can be obtained from Table A.1:

1. The $D=2$ contributions were generated using input $m_{s}(2 \mathrm{GeV})=115.4 \mathrm{MeV}$ for historical reasons. To get the corresponding contributions for any other $m_{s}(2 \mathrm{GeV})$ 


\begin{tabular}{|c||c|c|c|c|}
\hline Weight case & $s_{0}\left[\mathrm{GeV}^{2}\right]$ & $D=2$ & $D=4$ & $D=6$ \\
\hline \hline$(0,0)$ & 1.95 & 0.001669 & 0.000387 & -0.000040 \\
& 2.15 & 0.001824 & 0.000325 & -0.000033 \\
& 2.35 & 0.001930 & 0.000278 & -0.000028 \\
& 2.55 & 0.002005 & 0.000241 & -0.000024 \\
& 2.75 & 0.002057 & 0.000212 & -0.000020 \\
& 2.95 & 0.002093 & 0.000188 & -0.000018 \\
& 3.15 & 0.002119 & 0.000168 & -0.000015 \\
\hline \multirow{2}{*}{$w_{10}$} & 1.95 & 0.004019 & 0.001427 & -0.000013 \\
& 2.15 & 0.003900 & 0.001292 & -0.000011 \\
& 2.35 & 0.003792 & 0.001179 & -0.000009 \\
& 2.55 & 0.003694 & 0.001084 & -0.000008 \\
& 2.75 & 0.003605 & 0.001003 & -0.000007 \\
& 2.95 & 0.003524 & 0.000934 & -0.000006 \\
& 3.15 & 0.003449 & 0.000873 & -0.000005 \\
\hline
\end{tabular}

Table A.1: Integrated OPE D =2, 4, 6 contributions to the correlator difference for different $s_{0}$ and two weight cases.

value, simply rescale by the square of the new input value divided by 115.4 , e.g. for $m_{s}(2 \mathrm{GeV})=110 \mathrm{MeV}$, the tabulated values need to be rescaled by a multiplicative factor of:

$$
\left(\frac{110}{115.4}\right)^{2}=0.9084
$$

2. The $D=4$ contributions are associated with various products of quark masses and condensates (up to numerically tiny $m_{s}^{3} m_{u, d}$ corrections. These are determined by: (a) the Gell-Mann-Oakes-Renner (GMOR) relation for $\left\langle\left(m_{d}+m_{u}\right) u \bar{u}\right\rangle$ (known to be very accurate); (b) the known ratio of $m_{s}$ to $\left(m_{u}+m_{d}\right)$ [152], and; (c) the ratio $\langle s \bar{s}\rangle /\langle u \bar{u}\rangle$ (input above). Changing the input $m_{s}$ value therefore does not change the $D=4$ contributions.

3. Similarly, the $D=6$ contributions are not changed when the input $m_{s}$ value is changed (and they are very tiny anyway). 
Thus, the integrated OPE for any input $m_{s}(2 \mathrm{GeV})$ is obtained from the table entries via $\left(\frac{m_{s}(2 \mathrm{GeV})}{115.4}\right)^{2} \times[$ Table $D=2$ entry $]+[$ Table $D=4$ entry $]+[$ Table $D=6$ entry $]$.

In order to understand the meaning of Table A.1 for the analysis one has to understand the corresponding spectral integral of the flavour $u d V+A,(J=0)+(J=1)$ sum. (The corresponding flavour us sum spectral integrals are subtracted from the $u d$ integrals to get the flavour breaking difference used to get at $m_{s}$.) The corresponding $u d$ OPE integral has mass corrections dependent on $m_{u}$ and $m_{d}$, which are numerically tiny, and hence is dominated by its perturbative contribution ( $D=0$ ), with a quite small $D=4$ contribution due to the gluon condensate term. The $D=0$ term and the gluon condensate term are the same for the us OPE integrals and hence cancel in the difference. The size of the $u d$ spectral integral on its own, relative to the $u d-u s$ residual (which corresponds to the OPE integrals) above tells you the degree of cancellation expected when you form the $u d-u s$ spectral integral difference, as a function of $m_{s}$, and hence how well errors must be under control to make the analysis useful. The tabulated values in Table A.1 correspond to the integrals over the spectral function difference with no extra factors of $\pi, S_{\mathrm{EW}}, m_{\tau}$, etc. They are therefore not the same as $\Delta R_{\tau}$ (Eq. (2.33)), but constant multiples thereof. The corresponding $u d$ spectral integrals are given in Table A.2.

\section{A.4 Convergence behaviour of the OPE $D=2$ integrals}

The OPE for the $D=2$ part of the $\prod_{V+A ; u d}^{(0+1)}(s)-\prod_{V+A ; u s}^{(0+1)}(s)$ difference is a series of the form a running mass squared factor times a series in powers of the running coupling. One can integrate the terms in the resulting series separately order by order in the running coupling in order to see how well the integrated series converges with increasing order for the weight being used. Below these integrals are tabulated up to fourth order. The coefficients are known exactly up to second order, and the third and fourth order coefficients have been estimated in [150] using methods which work pretty well in known cases. From 


\begin{tabular}{|c||c|c|}
\hline Weight case & $s_{0}\left[\mathrm{GeV}^{2}\right]$ & $u d$ spectral integral $\left[\mathrm{GeV}^{2}\right]$ \\
\hline \hline$(0,0)$ & 1.95 & 0.06150 \\
& 2.15 & 0.06736 \\
& 2.35 & 0.07313 \\
& 2.55 & 0.07886 \\
& 2.75 & 0.08454 \\
& 2.95 & 0.09021 \\
& 3.15 & 0.09588 \\
\hline \multirow{3}{*}{$w_{10}$} & 1.95 & 0.04780 \\
& 2.15 & 0.05231 \\
& 2.35 & 0.05677 \\
& 2.55 & 0.06119 \\
& 2.75 & 0.06557 \\
& 2.95 & 0.06992 \\
& 3.15 & 0.07425 \\
\hline
\end{tabular}

Table A.2: ud spectral integrals for different $s_{0}$ and two weight cases. When $s_{0}=m_{\tau}^{2}$ this is the $R_{\tau}$ integral, up to constant factors.

Table A.3 it can be seen that the convergence does not look very convincing for the $(0,0)$ weight case (despite what is sometimes implied in the literature). Maltman therefore suggests that one should be very sceptical of results based on it. Comparing Table A.4 with Table A.3 it is clear that the convergence behaviour of the integrated series for the $w_{10}$ weight is much better (this was achieved by constructing $w_{10}$ in such a way as to bring the convergence problem under control). The results shown are the order $0,1,2,3,4$ integrated terms for the central OPE input cited above, as a function of $s_{0}$. All entries are in natural units ${ }^{1}$ of $\mathrm{GeV}^{2}$ and $a \equiv \alpha_{S} / \pi$ is used as the natural expansion parameter.

\footnotetext{
${ }^{1}$ In natural units $c=1$, therefore easing the notation.
} 


\begin{tabular}{|c||c|c|c|c|c|}
\hline$s_{0}$ & $\mathcal{O}\left(a^{0}\right)$ & $\mathcal{O}(a)$ & $\mathcal{O}\left(a^{2}\right)$ & $\mathcal{O}\left(a^{3}\right)$ & $\mathcal{O}\left(a^{4}\right)$ \\
\hline \hline 1.95 & 0.001873 & 0.000153 & -0.000357 & -0.001055 & -0.001874 \\
2.15 & 0.001889 & 0.000190 & -0.000256 & -0.000841 & -0.001511 \\
2.35 & 0.001894 & 0.000217 & -0.000181 & -0.000682 & -0.001243 \\
2.55 & 0.001894 & 0.000236 & -0.000125 & -0.000561 & -0.001039 \\
2.75 & 0.001889 & 0.000249 & -0.000082 & -0.000467 & -0.000881 \\
2.95 & 0.001882 & 0.000259 & -0.000048 & -0.000392 & -0.000756 \\
3.15 & 0.001873 & 0.000267 & -0.000021 & -0.000332 & -0.000656 \\
\hline
\end{tabular}

Table A.3: $D=2$ integrated OPE convergence vs $s_{0}$ for the $(0,0)$ weight.

\begin{tabular}{|c||c|c|c|c|c|}
\hline$s_{0}$ & $\mathcal{O}\left(a^{0}\right)$ & $\mathcal{O}(a)$ & $\mathcal{O}\left(a^{2}\right)$ & $\mathcal{O}\left(a^{3}\right)$ & $\mathcal{O}\left(a^{4}\right)$ \\
\hline \hline 1.95 & 0.002830 & 0.000698 & 0.000491 & 0.000264 & -0.000043 \\
2.15 & 0.002751 & 0.000672 & 0.000477 & 0.000276 & 0.000013 \\
2.35 & 0.002682 & 0.000648 & 0.000462 & 0.000281 & 0.000050 \\
2.55 & 0.002620 & 0.000626 & 0.000447 & 0.000282 & 0.000075 \\
2.75 & 0.002565 & 0.000607 & 0.000433 & 0.000280 & 0.000092 \\
2.95 & 0.002514 & 0.000589 & 0.000420 & 0.000276 & 0.000104 \\
3.15 & 0.002469 & 0.000573 & 0.000408 & 0.000271 & 0.000112 \\
\hline
\end{tabular}

Table A.4: $D=2$ integrated OPE convergence $v s s_{0}$ for the $w_{10}$ weight. 


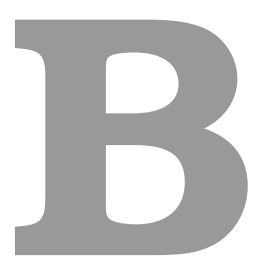

\section{KK2 $f$ Monte Carlo branching fractions}

Table B.1 lists the decay modes and corresponding branching fractions used as input into the $\tau^{+} \tau^{-}$event generator KK2 $\mathrm{f}$ (with TAUOLA) in SP5 ${ }^{1}$. They are based on the PDG 2002 averages (not fitted values) [39]. The quoted values have been calculated with an imposed unitarity constraint, which has resulted in all modes increasing by about $0.4 \sigma$ above the PDG 2002 averages.

Mode 7 contains the signal mode under discussion in this thesis. For this mode, the only lineshape used is that of $\tau^{-} \rightarrow K^{*}(892)^{-} \nu_{\tau}$ decay: a Breit-Wigner amplitude is used for this $p$-wave resonance. Higher $K^{*}$ resonances $\left(K^{*}(1410)^{-}, K_{0}^{*}(1430)^{-}, K^{*}(1680)^{-}\right)$, are not simulated in the Monte Carlo, so there is no simulated interference between $K^{*}$ resonances.

By default, modes 23-28 are "turned off" (i.e. have zero branching fraction) so generics are not made with these modes, but they can be "turned on" to generate signal Monte

\footnotetext{
${ }^{1}$ Caveat: the level of precision input into the generator is not the same for each decay mode. This can be checked by looking at the .Kk2f_defaults file used by the KK2 $f$ generator in the appropriate production release.
} 
Carlo for any future studies of these decay modes.

\begin{tabular}{|c|c|c|}
\hline Mode number & Decay mode & $\mathcal{B}$ \\
\hline \hline 1 & $\tau^{-} \rightarrow e^{-} \bar{\nu}_{e} \nu_{\tau}$ & 0.178651 \\
2 & $\tau^{-} \rightarrow \mu^{-} \bar{\nu}_{\mu} \nu_{\tau}$ & 0.173551 \\
3 & $\tau^{-} \rightarrow \pi^{-} \nu_{\tau}$ & 0.110841 \\
4 & $\tau^{-} \rightarrow \pi^{-} \pi^{0} \nu_{\tau}$ & 0.253754 \\
5 & $\tau^{-} \rightarrow a_{1}^{-} \nu_{\tau}\left(2 \pi^{-} \pi^{+}=9.1902 \% ; \pi^{-} 2 \pi^{0}=9.1664 \%\right)$ & 0.183566 \\
6 & $\tau^{-} \rightarrow K^{-} \nu_{\tau}$ & 0.006946 \\
7 & $\tau^{-} \rightarrow K^{*-} \nu_{\tau}\left(K^{-} \pi^{0}=0.46 \% ; \bar{K}^{0} \pi^{-}=0.90 \%\right)$ & 0.013641 \\
8 & $\tau^{-} \rightarrow 2 \pi^{-} \pi^{0} \pi^{+} \nu_{\tau}$ & 0.043654 \\
9 & $\tau^{-} \rightarrow 3 \pi^{0} \pi^{-} \nu_{\tau}$ & 0.012619 \\
10 & $\tau^{-} \pi^{+} 2 \pi^{0} \nu_{\tau}\left(\mathrm{excl} . \eta \pi^{-} \pi\right)$ & 0.005011 \\
11 & $\tau^{-} \rightarrow 3 \pi^{-} 2 \pi^{+} \nu_{\tau}$ & 0.000789 \\
12 & $\tau^{-} \rightarrow 3 \pi^{-} 2 \pi^{+} \pi^{0} \nu_{\tau}$ & 0.000183 \\
13 & $\tau^{-} \rightarrow 2 \pi^{-} \pi^{+} 3 \pi^{0} \nu_{\tau}$ & 0.000251 \\
14 & $\tau^{-} \rightarrow K^{-} \pi^{-} K^{+} \nu_{\tau}$ & 0.001590 \\
15 & $\tau^{-} \rightarrow K^{0} \pi^{-} \bar{K}^{0} \nu_{\tau}$ & 0.001672 \\
16 & $\tau^{-} \rightarrow K^{-} K^{0} \pi^{0} \nu_{\tau}$ & 0.001536 \\
17 & $\tau^{-} \rightarrow 2 \pi^{0} K^{-} \nu_{\tau}$ & 0.000680 \\
18 & $\tau^{-} \rightarrow K^{-} \pi^{-} \pi^{+} \nu_{\tau}$ & 0.003009 \\
19 & $\tau^{-} \rightarrow \pi^{-} \bar{K}^{0} \pi^{0} \nu_{\tau}$ & 0.003767 \\
20 & $\tau^{-} \rightarrow \eta \pi^{-} \pi^{0} \nu_{\tau}$ & 0.001830 \\
21 & $\tau^{-} \rightarrow \pi^{-} \pi^{0} \gamma \nu_{\tau}$ & 0.000802 \\
22 & $\tau^{-} \rightarrow K^{-} K^{0} \nu_{\tau}$ & 0.001651 \\
23 & $\tau^{-} \rightarrow 4 \pi^{-} 3 \pi^{+} \nu_{\tau}$ & 0.000000 \\
24 & $\tau^{-} \rightarrow 4 \pi^{-} 3 \pi^{+} \pi^{0} \nu_{\tau}$ & 0.000000 \\
25 & $\tau^{-} \rightarrow e^{-} \gamma$ & 0.000000 \\
26 & $\tau^{-} \rightarrow \mu^{-} \gamma$ & 0.000000 \\
27 & $\tau^{-} \rightarrow \pi^{-} \pi^{0} \nu_{\tau}(\mathrm{CPV})$ & 0.000000 \\
28 & $\tau^{-} \rightarrow(K \pi)^{-} \nu_{\tau}(\mathrm{CPV})$ & 0.000000 \\
\hline
\end{tabular}

Table B.1: KK2f decay modes and branching fractions based on the PDG 2002 averages with unitarity constraint. The highlighted row contains the signal mode under discussion in this document. An archived copy of these values can be found here [153]. Note that the precision level input into the generator varies between modes (see. $\mathrm{Kk} 2 \mathrm{f} \_d e f a u l t s$ file in the production release as necessary). 


\section{References}

[1] Nucl. Phys. B. Proc. Suppl. 123 (2003). Proceedings of the $7^{\text {th }}$ international workshop on $\tau$ lepton physics, 10-13 September 2002, Santa Cruz, CA, USA.

[2] K. Maltman, The strange quark mass, $\alpha_{S}$ and the chiral limit electroweak penguin $K \rightarrow \pi \pi$ matrix elements from hadronic $\tau$ decay data, hep-ph/0209091.

[3] Elba 2003, BABAR collaboration meeting, 20-23 May 2003, Isola d'Elba, Italy.

[4] S. L. Glashow, Partial symmetries of weak interactions, Nucl. Phys. 22 (1961) $579-588$.

[5] S. Weinberg, A model of leptons, Phys. Rev. Lett. 19 (1967) 1264-1266.

[6] A. Salam and e. Swartholm, N. In Proc. $8^{\text {th }}$ Nobel Symp. (1968).

[7] N. Cabibbo, Unitary symmetry and leptonic decays, Phys. Rev. Lett. 10 (1963) $531-532$.

[8] M. Kobayashi and T. Maskawa, CP violation in the renormalizable theory of weak interaction, Prog. Theor. Phys. 49 (1973) 652-657.

[9] A. Stahl, Physics with Tau Leptons. Springer-Verlag, New York, 2000.

[10] R. Gupta and K. Maltman, Light quark masses: A status report at DPF 2000, Int. J. Mod. Phys. A16S1B (2001) 591-600 [hep-ph / 0101132$].$

[11] ALEPH Collaboration, M. Davier and C.-z. Yuan, Measurement of branching fractions in tau decays, eConf C0209101 (2002) TU06 [hep-ex/0211057]. 
[12] M. Davier, Spectral functions from hadronic $\tau$ decays and $Q C D$, eConf $\mathbf{C 0 2 0 9 1 0 1}$ (2002) WE03 [hep-ex/0301035].

[13] A. Pich, Leptonic probes of the standard model, eConf C0209101 (2002) TU01 [hep-ph/0210445].

[14] ALEPH Collaboration, D. Buskulic et. al., Tau hadronic branching ratios, $Z$. Phys. C70 (1996) 579-608.

[15] ALEPH Collaboration, R. Barate et. al., Measurement of the spectral functions of vector current hadronic $\tau$ decays, Z. Phys. C76 (1997) 15-33.

[16] ALEPH Collaboration, R. Barate et. al., $K_{S}^{0}$ production in $\tau$ decays, Eur. Phys. J. C4 (1998) 29-45.

[17] ALEPH Collaboration, R. Barate et. al., Measurement of the spectral functions of axial-vector hadronic $\tau$ decays and determination of $\alpha_{S}\left(m_{\tau}^{2}\right)$, Eur. Phys. J. C4 (1998) 409-431.

[18] ALEPH Collaboration, R. Barate et. al., Three-prong tau decays with charged kaons, Eur. Phys. J. C1 (1998) 65-79.

[19] ALEPH Collaboration, R. Barate et. al., One-prong tau decays with kaons, Eur. Phys. J. C10 (1999) 1-18 [hep-ex/9903014].

[20] ALEPH Collaboration, R. Barate et. al., Study of $\tau$ decays involving kaons, spectral functions and determination of the strange quark mass, Eur. Phys. J. C11 (1999) 599-618 [hep-ex/9903015].

[21] S. Chen et. al., Strange quark mass from the invariant mass distribution of Cabibbo-suppressed $\tau$ decays, Eur. Phys. J. C22 (2001) 31-38 [hep-ph/0105253].

[22] CLEO Collaboration, M. Goldberg et. al., Study of $K^{*}$ production in tau decay, Phys. Lett. B251 (1990) 223-228. 
[23] CLEO Collaboration, M. Athanas et. al., Study of the decays $\tau^{-} \rightarrow K_{S}^{0} h^{-}\left(\pi^{0}\right) \nu_{\tau}$, CLEO-CONF 94-23, 1994.

[24] CLEO Collaboration, T. Coan et. al., Measurement of $\alpha_{S}$ from tau decays, Phys. Lett. B356 (1995) 580-588.

[25] CLEO Collaboration, J. P. Alexander et. al., "Measurement of hadronic spectral moments in tau decays and a determination of $\alpha_{S}$." Submitted to Int. Conf. on High Energy Physics, Glasgow, Scotland, Jul 20-27, 1994.

[26] CLEO Collaboration, T. E. Coan et. al., Decays of $\tau$ leptons to fi nal states containing $K_{S}^{0}$ mesons, Phys. Rev. D53 (1996) 6037-6053.

[27] CLEO Collaboration, S. J. Richichi et. al., Study of 3-prong hadronic $\tau$ decays with charged kaons, Phys. Rev. D60 (1999) 112002 [hep-ex/9810026].

[28] CLEO Collaboration, A. J. Weinstein, Semi-hadronic tau decays at CLEO, Nucl. Phys. Proc. Suppl. 98 (2001) 261-280 [hep-ex/ 0012064$].$

[29] CLEO Collaboration, R. A. Briere et. al., Branching fractions of tau lepton decays to three charged hadrons, Phys. Rev. Lett. 90 (2003) 181802 [hep-ex/0302028].

[30] OPAL Collaboration, R. Akers et. al., Measurements of the inclusive branching ratios of tau leptons to $K_{S}^{0}$ and charged $K^{*}(892)$, Phys. Lett. B339 (1994) $278-292$.

[31] OPAL Collaboration, K. Ackerstaff et. al., Measurement of the strong coupling constant $\alpha_{S}$ and the vector and axial-vector spectral functions in hadronic tau decays, Eur. Phys. J. C7 (1999) 571-593 [hep-ex/9808019].

[32] OPAL Collaboration, G. Abbiendi et. al., A study of three-prong tau decays with charged kaons, Eur. Phys. J. C13 (2000) 197-212 [hep-ex/9908013].

[33] OPAL Collaboration, G. Abbiendi et. al., Tau decays with neutral kaons, Eur. Phys. J. C13 (2000) 213-223 [hep-ex/9911029]. 
[34] OPAL Collaboration, G. Abbiendi et. al., A study of one-prong tau decays with a charged kaon, Eur. Phys. J. C19 (2001) 653-665 [hep-ex/0 009017$].$

[35] K. Maltman and J. Kambor, On the longitudinal contributions to hadronic $\tau$ decay, Phys. Rev. D64 (2001) 093014 [hep-ph / 0107187$].$

[36] Y. S. Tsai, Search for new mechanism of CP violation through tau decay and semileptonic decay of hadrons, Nucl. Phys. Proc. Suppl. 55C (1997) 293-304 [hep-ph/9612281].

[37] CLEO Collaboration, G. Bonvicini et. al., Search for CP violation in $\tau \rightarrow K \pi \nu_{\tau}$ decays, Phys. Rev. Lett. 88 (2002) 111803 [hep-ex/0111095].

[38] M. L. Perl et. al., Evidence for anomalous lepton production in $e^{+} e^{-}$annihilation, Phys. Rev. Lett. 35 (1975) 1489-1492.

[39] Particle Data Group Collaboration, K. Hagiwara et. al., Review of particle physics, Phys. Rev. D66 (2002) 010001.

[40] M. L. Perl, Tau physics, 1992. Presented at $20^{\text {th }}$ Annual SLAC Summer Institute on Particle Physics: The Third Family and the Physics of Flavor (School: Jul 13-24, Topical Conference: Jul 22-24, Symposium on Tau Physics: Jul 24) (SSI 92), Stanford, CA, 13-24 Jul 1992.

[41] S. Mandelstam, Determination of the pion-nucleon scattering amplitude from dispersion relations and unitarity, Phys. Rev. 112 (1958) 1344.

[42] A. Lusiani and E. Paoloni, "Measurement of the $\tau$ lepton lifetime using the decay length method." BAD \#55, Version 12 (BABAR internal analysis document), 2003.

[43] A. J. Buras, Weak hamiltonian, CP violation and rare decays, 1998. hep-ph/9806471.

[44] A. J. Buras, Operator product expansion, renormalization group and weak decays, Lect. Notes Phys. 558 (2000) 65-85 [hep-ph/9901409]. 
[45] A. J. Buras, M. Jamin and M. E. Lautenbacher, The anatomy of $\varepsilon^{\prime} / \varepsilon$ beyond leading logarithms with improved hadronic matrix elements, Nucl. Phys. B408 (1993) 209-285 [hep-ph/9303284].

[46] A. J. Buras and M. E. Lautenbacher, An analytic formula and an upper bound for $\varepsilon^{\prime} / \varepsilon$ in the standard model, Phys. Lett. B318 (1993) 212-216 [hep-ph/9309203].

[47] M. Ciuchini, E. Franco, G. Martinelli and L. Reina, The $\Delta S=1$ effective hamiltonian including next-to-leading order QCD and QED corrections, Nucl. Phys. B415 (1994) 403-462 [hep-ph / 9304257$].$

[48] C. E. Wolfe and K. Maltman, The strong isospin breaking correction for the gluonic penguin contribution to $\varepsilon^{\prime} / \varepsilon$ at next-to-leading order in the chiral expansion, Phys. Rev. D63 (2001) 014008 [hep-ph/ 0007319$].$

[49] K. Maltman and J. Kambor, Optimizing the FESR extraction of $m_{s}$ from hadronic

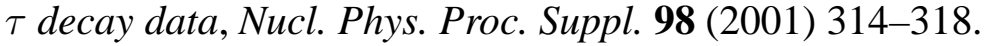

[50] L.-L. Chau and W.-Y. Keung, Comments on the parametrization of the Kobayashi-Maskawa matrix, Phys. Rev. Lett. 53 (1984) 1802.

[51] BABAR Collaboration, B. Aubert et. al., Observation of $C P$ violation in the $B^{0}$ meson system, Phys. Rev. Lett. 87 (2001) 091801 [hep-ex/0107013].

[52] BABAR Collaboration, e. Harrison, P. F. and e. Quinn, Helen R., The BABAR physics book: Physics at an asymmetric B Factory, 1998. Papers from Workshop on Physics at an asymmetric $B$ Factory (BABAR Collaboration Meeting), Rome, Italy, 11-14 Nov 1996, Princeton, NJ, 17-20 Mar 1997, Orsay, France, 16-19 Jun 1997 and Pasadena, CA, 22-24 Sep 1997.

[53] M. Finkemeier and E. Mirkes, Decay rates, structure functions and new physics effects in hadronic tau decays, 1995. hep-ph/9508312. 
[54] J. H. Kühn and E. Mirkes, Structure functions in tau decays, Z. Phys. C56 (1992) $661-672$.

[55] Y.-S. Tsai, Decay correlations of heavy leptons in $e^{+} e^{-} \rightarrow l^{+} l^{-}$, Phys. Rev. D4 (1971) 2821.

[56] M. L. Perl, The tau lepton, Rept. Prog. Phys. 55 (1992) 653-722.

[57] J. H. Kühn and A. Santamaria, Tau decays to pions, Z. Phys. C48 (1990) 445-452.

[58] G. J. Gounaris and J. J. Sakurai, Finite width corrections to the vector meson dominance prediction for $\rho \rightarrow e^{+} e^{-}$, Phys. Rev. Lett. 21 (1968) 244.

[59] J. Fulwood, A study of $\tau^{-} \rightarrow \pi^{-} \pi^{0} \nu_{\tau}$ using the BABAR detector. PhD thesis, Manchester University, 2001.

[60] J. Fulwood, G. Lafferty and J. Williams, "A study of $\tau^{-} \rightarrow \pi^{-} \pi^{0} \nu_{\tau}$ using the BABAR detector.” BAD \#185, Version 1 (BABAR internal analysis document), 2001.

[61] LASS Collaboration, D. Aston et. al., A study of $K^{-} \pi^{+}$scattering in the reaction $K^{-} p \rightarrow K^{-} \pi^{+} n$ at $11 \mathrm{GeV} / c$, Nucl. Phys. $\mathbf{3 2 9 6}$ (1988) 493.

[62] W. Dunwoodie, “ $K \pi s$-wave fit note." http: / /www.slac.stanford.edu/ 〜wm/kpi_swave/kpi_swave_fit.note.

[63] A. V. Anisovich and A. V. Sarantsev, $K$-matrix analysis of the $K \pi s$-wave in the mass region $900 \mathrm{MeV}$ to $2100 \mathrm{MeV}$ and nonet classifi cation of scalar $\overline{q q}$ states, Phys. Lett. B413 (1997) 137-146 [hep-ph/9705401].

[64] J. Fischer and I. Vrkoc, Operator product expansion and analyticity, Int. J. Mod. Phys. A14 (1999) 4819-4840 [hep-ph/9908484].

[65] K. G. Wilson, Nonlagrangian models of current algebra, Phys. Rev. 179 (1969) 1499-1512. 
[66] W. Zimmermann, Lectures on Elementary Particles and Quantum Field Theory, ch. Local operator products and renormalization in quantum field theory, pp. 395-589. MIT Press, 1970.

[67] M. A. Shifman, A. I. Vainshtein and V. I. Zakharov, QCD and resonance physics. Sum rules., Nucl. Phys. B147 (1979) 385-447.

[68] E. Braaten, S. Narison and A. Pich, QCD analysis of the tau hadronic width, Nucl. Phys. B373 (1992) 581-612.

[69] http: / /www.slac.stanford.edu/BFROOT/www/Organization/ CollabMtgs/2003/detMay2003/Wed1d/MatthiasJamin.pdf.

[70] A. Pich, QCD tests from $\tau$ decays, 1997. hep-ph/9701305.

[71] V. Cirigliano, E. Golowich and K. Maltman, QCD condensates for the light quark $V$-A correlator, hep-ph/0305118.

[72] E. C. Poggio, H. R. Quinn and S. Weinberg, Smearing the quark model, Phys. Rev. D13 (1976) 1958.

[73] E. Gamiz, M. Jamin, A. Pich, J. Prades and F. Schwab, Determination of $m_{s}$ and $\left|V_{u s}\right|$ from hadronic $\tau$ decays, JHEP 01 (2003) 060 [hep-ph/ 0212230$]$.

[74] M. Finkemeier and E. Mirkes, The scalar contribution to $\tau \rightarrow K \pi \nu_{\tau}$, Z. Phys. C72 (1996) 619-626 [hep-ph/9601275].

[75] E791 Collaboration, E. M. Aitala et. al., Dalitz plot analysis of the decay $D^{+} \rightarrow K^{-} \pi^{+} \pi^{+}$and study of the K $\pi$ scalar amplitudes, Phys. Rev. Lett. 89 (2002) 121801 [hep-ex/0204018].

[76] M. Jamin, J. A. Oller and A. Pich, s-wave $K \pi$ scattering in chiral perturbation theory with resonances, Nucl. Phys. B587 (2000) 331-362 [hep-ph/ 0006045$].$

[77] M. Jamin, J. A. Oller and A. Pich, Strangeness-changing scalar form factors, Nucl. Phys. B622 (2002) 279-308 [hep-ph/ 0110193$].$ 
[78] M. Jamin. Private communication, 12 June 2003.

[79] M. Jamin. Private communication, 12 September 2003.

[80] PEP-II: An asymmetric B Factory. Conceptual design report, 1993. SLAC-418

[81] J. Seeman et. al., The PEP-II storage rings, 2001. SLAC-PUB-9350

[82] BELLE Collaboration, A. Bondar, The BELLE detector, Nucl. Instrum. Meth. A408 (1998) 64-76.

[83] BELLE Collaboration, A. E. Bondar, KEKB performance, Nucl. Instrum. Meth. A462 (2001) 139-145.

[84] http://www.slac.stanford.edu/BFROOT/www/Physics / BaBarData/BaBarData.html.

[85] BABAR Collaboration, D. Boutigny et. al., BABAR technical design report, 1995. SLAC-R-0457

[86] BABAR Collaboration, B. Aubert et. al., The BABAR detector, Nucl. Instrum. Meth. A479 (2002) 1-116 [hep-ex/0105044].

[87] BABAR Collaboration, D. Boutigny et. al., Letter of intent for the study of CP violation and heavy flavor physics at PEP-II, 1994. SLAC-0443

[88] A. Snyder, "Effect of vertex cuts on $C P$ reach." BABAR note \#177, Version 1 (BABAR internal document), 1994.

[89] B. R. Martin and G. Shaw, Particle Physics, pp. 53-55. John Wiley \& Sons, $2^{\text {nd }}$ ed., 1997.

[90] G. Raven, “Drift chamber tracking conventions.” BABAR note \#488, Version 1 (BABAR internal document), 1999.

[91] P. Billoir, Track fi tting with multiple scattering: a new method, Nucl. Instr. Meth. A225 (1984) 352. 
[92] Objectivity Inc., Mountain View, CA, USA.

[93] http: / /www.slac.stanford.edu/BFROOT/www/Computing/ Offline/Kanga/.

[94] http: / /www.slac.stanford.edu/BFROOT/www/Computing/.

[95] B. Stroustrup, The C++ Programming Language. Addison-Wesley, $3^{\text {rd }}$ ed., 1997.

[96] http: //www.research.att.com/ bs/C++.html.

[97] http: / /www. cvshome.org/.

[98] http: / /www.openafs.org/.

[99] B. Welch, Practical Programming in TCL and TK. Prentice Hall PTR, 1999.

[100] http://www.slac.stanford.edu/BFROOT/dist/releases/ newest/Elf/README.

[101] http: / /www.slac.stanford.edu/BFROOT/www/Computing/ Offline/Beta/Beta.html.

[102] http://www.slac.stanford.edu/BFROOT/www/Physics/Tools/ Vertex/CompGuide/.

[103] U. Langenegger, “Cut-based electron identification.” BAD \#90, Version 5, 2001.

[104] L. Lista, "Muon identification in the BABAR experiment." BAD \#60, Version 1, 2000.

[105] http://www.slac.stanford.edu/BFROOT/www/Physics/Tools/ Pid/Hadrons/Description_of_the_LH_selectors.html.

[106] S. Spanier and G. Mancinelli, "Kaon selection at BABAR." BAD \#116, Version 1, 2001. 
[107] http://www.slac.stanford.edu/BFROOT/www/Physics/Tools/ Pid/Ntuples/manual.html.

[108] http://www.slac.stanford.edu/BFROOT/www/Physics/Tools/ Pid/PidKilling/PidKilling.html.

[109] http: / /www.slac.stanford.edu/BFROOT/www/doc/workbook/ sim/sim.html.

[110] D. Lange and A. Ryd, "EvtGen documentation.” BAD \#522, Version 6, (BABAR internal analysis document), 2003.

[111] S. Jadach and Z. Was, KORALB: An upgrade to version 2.4, Comput. Phys. Commun. 85 (1995) 453-462.

[112] B. F. L. Ward, S. Jadach and Z. Was, Precision calculation for $e^{+} e^{-} \rightarrow 2 f:$ The KKMC project, Nucl. Phys. Proc. Suppl. 116 (2003) 73-77

[hep-ph/0211132].

[113] Z. Was, TAUOLA the library for tau lepton decay, and KKMC/KORALB/KORALZ/... status report, Nucl. Phys. Proc. Suppl. 98 (2001) 96-102 [hep-ph/0011305].

[114] http://www.slac.stanford.edu/BFROOT/www/Physics/Tools/ generators/GenFwkInt/GenFwkInt.html.

[115] http://www.slac.stanford.edu/BFROOT/www/Computing/ Offline/Simulation/web/bbsim-guide.html.

[116] GEANT4 Collaboration, S. Agostinelli et. al., GEANT4: A simulation toolkit, Nucl. Instr. Meth. A506 (2003) 250-303.

[117] http://wwwinfo.cern.ch/asd/geant4/geant4.html.

[118] BABAR Computing Group Collaboration, D. H. Wright et. al., Using GEANT4 in the BABAR simulation, CHEP 2003. hep-ph/0305240. 
[119] N. Barlow et. al., "Improvement of background mixing in all subsystems in the BABAR Monte Carlo simulation.” BAD \#332, Version 2 (BABAR internal analysis document), 2002.

[120] BABAR Collaboration, D. N. Brown, The BABAR Mini, hep-ex/0305085.

[121] http://www.slac.stanford.edu/BEROOT/wWw/doc/workbook/ nanomicro/v8.8/.

[122] J. Williams, “TauUser.” BAD \#842, Version 1 (BABAR internal analysis document), 2004.

[123] http://www.slac.stanford.edu/BFROOT/www/Physics/ Analysis/tauqed/TauUser/TauUserIntro.html.

[124] http://root.cern.ch/.

[125] http://wwwasd.web.cern. ch/wwwasd/paw/.

[126] R. J. Barlow, Statistics. John Wiley \& Sons, 1997.

[127] L. Lyons, Statistics for Nuclear and Particle Physicists. Cambridge University Press, 1989.

[128] G. Cowan, Statistical Data Analysis. Oxford University Press, 1998.

[129] R. J. Barlow, Extended maximum likelihood, Nucl. Instrum. Meth. A297 (1990) 496-506.

[130] F. James and M. Roos, MINUIT: A system for function minimization and analysis of the parameter errors and correlations, Comput. Phys. Commun. 10 (1975) $343-367$.

[131] http://roofit.sourceforge.net/.

[132] W. Verkerke and D. Kirkby, The RooFit toolkit for data modeling, eConf C0303241 (2003) MOLT007 [physics / 0306116 ]. 
[133] http://www.slac.stanford.edu/BFROOT/www/Physics/ SimProd/sp5inventory.html.

[134] S. Banerjee et. al., "Nano and micro level preselection for $e^{+} e^{-} \rightarrow \mu^{+} \mu^{-}$and $e^{+} e^{-} \rightarrow \tau^{+} \tau^{-}$events." See Table 1. BAD \#739, Version 2 (BABAR internal analysis document), 2003.

[135] F. Martinez-Vidal, “The BABAR vertexing." BAD \#102, Version 6 (BABAR internal analysis document), 2001.

[136] http://www.slac.stanford.edu/BFROOT/www/Physics/ TrackEfficTaskForce/Recipe/KsTracks-R10.html.

[137] A. Lyon, "PID selector performance in the $\tau^{-} \rightarrow(K \pi)^{-} \nu_{\tau}$ analysis." BAD \#843, Version 2 (BABAR internal analysis document), 2004.

[138] J. Allison, A. Lyon and F. Salvatore, "Measurement of $\mathcal{B}\left(\tau^{-} \rightarrow(K \pi)^{-} \nu_{\tau}\right)$ using the BABAR detector.” BAD \#829, Version 1 (BABAR internal analysis document), 2004.

[139] E. Varnes, "Report of the tracking efficiency task force." BAD \#324, Version 2, (BABAR internal analysis document), 2002.

[140] A. Telnov, " $K_{S}^{0} \rightarrow \pi^{+} \pi^{-}$reconstruction efficiency in BABAR: Release-10 data vs SP4 Monte Carlo.” BAD \#677, Version 3, (BABAR internal analysis document), 2003.

[141] J. Berryhill, "Measurement of the rare decays $B \rightarrow K l^{+} l^{-}$and $B \rightarrow K^{*} l^{+} l^{-}$." BAD \#559, Version 15, (BABAR internal analysis document), 2003.

[142] BABAR Collaboration, B. Aubert et. al., Evidence for the rare decay $B \rightarrow K^{*} l^{+} l^{-}$and measurement of the $B \rightarrow K l^{+} l^{-}$branching fraction, Phys. Rev. Lett. 91 (2003) 221802 [hep-ex/0308042]. 
[143] O. Igonkina, “A search for lepton flavor violating decays $\tau^{-} \rightarrow l^{-} l^{+} l^{-}$.” BAD \#722, Version 11, (BABAR internal analysis document), supporting document. BAD \#761, Version 15, (BABAR internal analysis document), submitted to PRL, 2003.

[144] M. Finkemeier and E. Mirkes, Tau decays into kaons, Z. Phys. C69 (1996) 243-252 [hep-ph/9503474].

[145] W. M. Dunwoodie. Private communication, 4 March 2004.

[146] LASS Collaboration, W. M. Dunwoodie, P. Rensing and L. Chen, Two variations on a $\rho^{\prime}$ theme: Data from LASS and MARK-III, . Presented at Joint Inst. Lepton-Photon Symp. and Europhysics Conf. on High Energy Physics, Geneva, Switzerland, Jul 25 - Aug 1, 1991.

[147] M. Boas, Mathematical Methods in the Physical Sciences, p. 487. John Wiley \& Sons, $2^{\text {nd }}$ ed., 1983.

[148] W. M. Dunwoodie. Private communication, 19 December 2003.

[149] K. Maltman. Private communication, 8 August 2003.

[150] P. A. Baikov, K. G. Chetyrkin and J. H. Kühn, Five-loop vacuum polarization in pQCD: $\mathcal{O}\left(m_{q}^{2} \alpha_{S}^{4} n_{f}^{2}\right)$ contribution, Phys. Lett. $\mathbf{B 5 5 9}$ (2003) 245-251 [hep-ph/0212303].

[151] P. A. Baikov, K. G. Chetyrkin and J. H. Kühn, Towards order $\alpha_{S}^{4}$ accuracy in tau decays, Phys. Rev. D67 (2003) 074026 [hep-ph / 0212299$].$

[152] H. Leutwyler, Non-lattice determinations of the light quark masses, Nucl. Phys. Proc. Suppl. 94 (2001) 108-115 [hep-ph/ 0011049 ].

[153] http://babar-hn.slac.stanford.edu: 5090 /HyperNews / get/TauQED/279/2 .html. 
Internal documents can be obtained with the permission of the BABAR collaboration. Please contact the author (andylyon@slac.stanford.edu) or another BABAR collaboration member for details. 